\title{
Molecular determinants of bacterial antimicrobial efflux by the Major Facilitator Superfamily members CraA and TetA from Acinetobacter baumannii
}

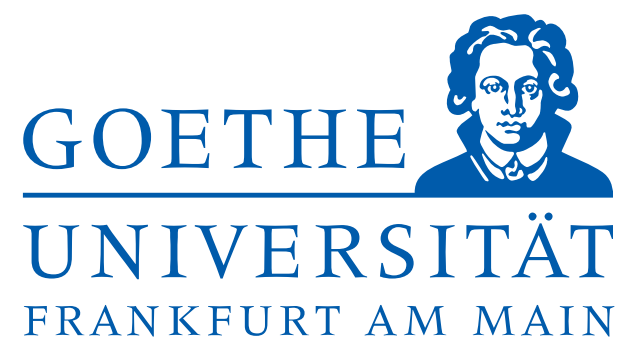

Dissertation

zur Erlangung des Doktorgrades

der Naturwissenschaften
vorgelegt im Fachbereich 14 Biochemie, Chemie und Pharmazie der Johann Wolfgang Goethe Universität
Frankfurt am Main

\author{
von \\ Wuen Ee Foong \\ aus Kuala Lumpur, Malaysia
}

Frankfurt am Main (2021)

(D30) 
Vom Fachbereich Biochemie, Chemie und Pharmazie (FB14)

der Johann Wolfgang Goethe Universität als Dissertation angenommen.

Dekan: Prof. Dr. Clemens Glaubitz

Gutachter: 1. Prof. Dr. Klaas Martinus Pos

2. Prof. Dr. Beate Averhoff

Datum der Disputation: 
Part of the work in this thesis has published in the below publications:

- Foong WE, Tam HK, Crames JJ, Averhoff B, Pos KM (2019) The chloramphenicol/ $\mathrm{H}^{+}$ antiporter CraA of Acinetobacter baumannii AYE reveals a broad substrate specificity. J. Antimicrob. Chemother. 74: 1192-1201.

- Foong WE, Wilhelm J, Tam HK, Pos KM (2020) Tigecycline efflux in Acinetobacter baumannii is mediated by TetA in synergy with RND-type efflux transporters. J. Antimicrob. Chemother. 75: 1135-1139. 


\section{TABLE OF CONTENTS}

Zusammenfassung

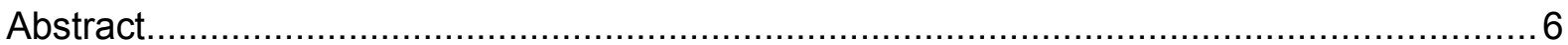

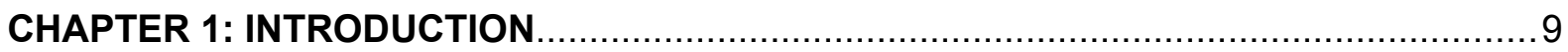

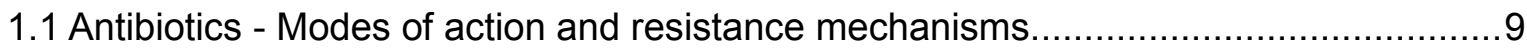

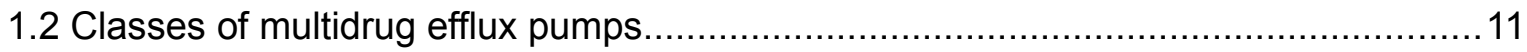

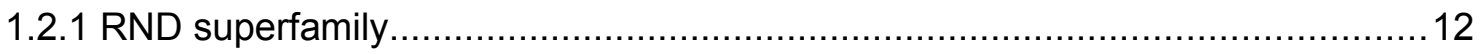

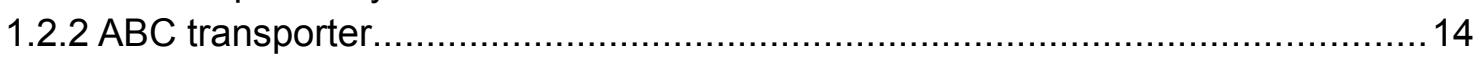

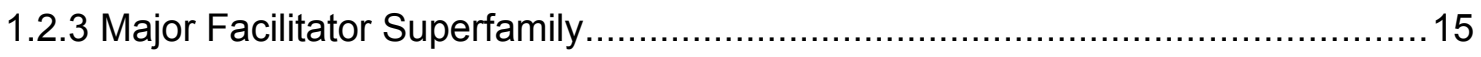

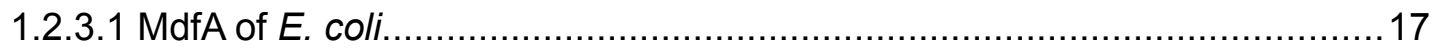

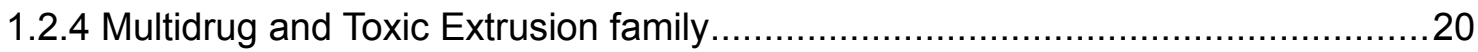

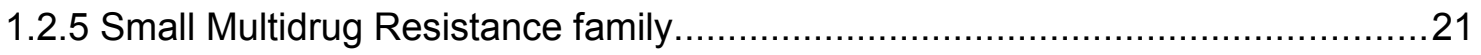

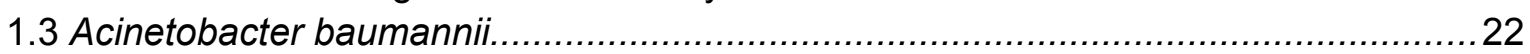

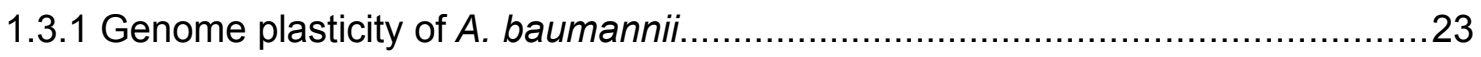

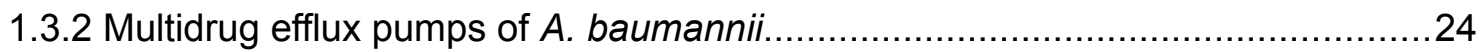

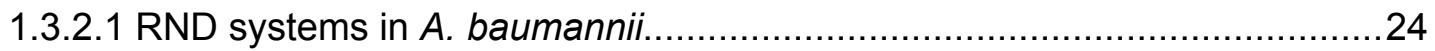

1.3.2.2 Other non-RND efflux pumps of $A$. baumannii......................................27

1.3.3 Outer membrane proteins of $A$. baumannii...............................................29

1.4 Resistance to the last-resort antibiotic tigecycline in $A$. baumannii............................31

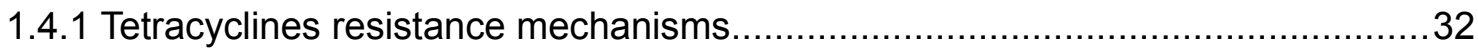

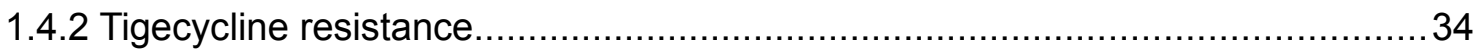

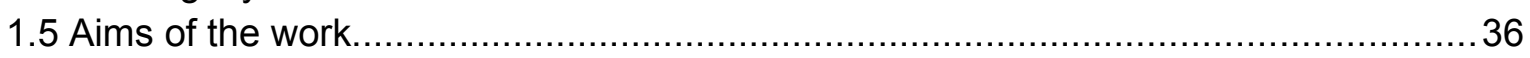

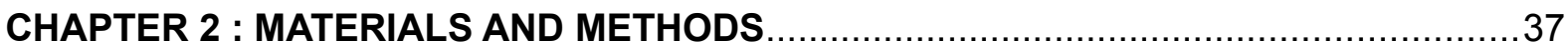

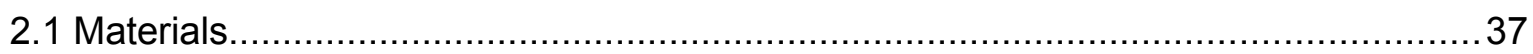

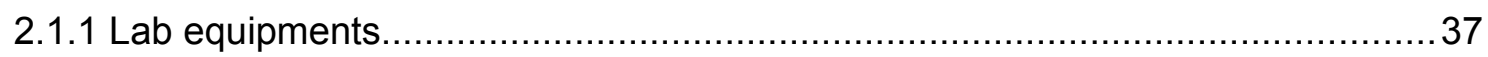

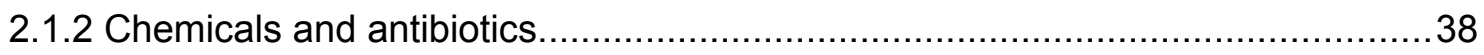

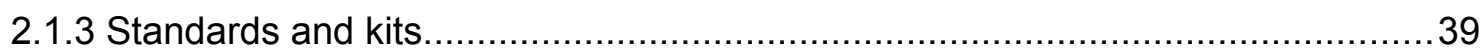

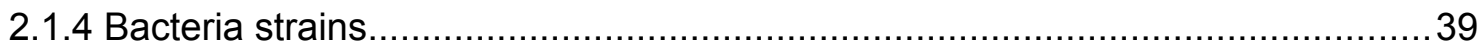

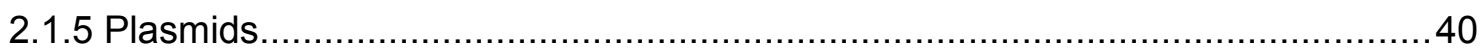

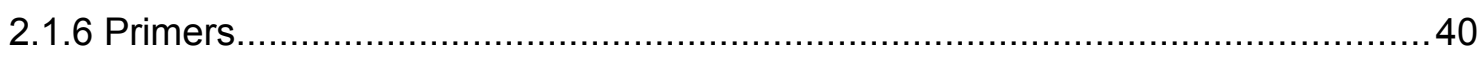

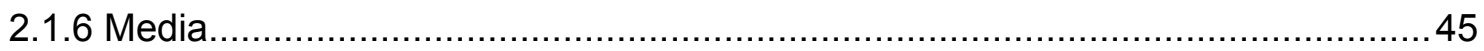

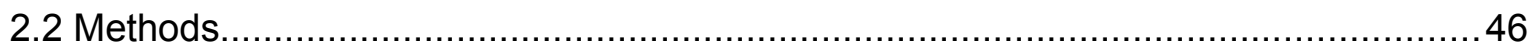

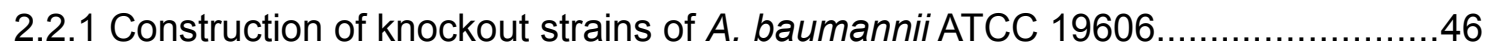

2.2.1.1 Cloning of plasmid (pBIISK_sacB/kanR_updown).................................46

2.2.1.2 Transformation and the first round of colony-PCR .................................47

2.2.1.3 Counter-selection using sucrose and second round of colony-PCR...........48

2.2.2 Differential gene expression analysis for $A$. baumannii knockout strains............48

2.2.2.1 Sample preparation and RNA extraction.............................................48

2.2.2.2 Quantitative reverse transcription PCR..................................................49

2.2.3 Cloning of putative chloramphenicol or tetracycline transporter genes..............50

2.2.4 PCR based site-directed mutagenesis of CraA .............................................. 50

2.2.5 Characterization of CraA of $A$. baumannii strain AYE.......................................52 


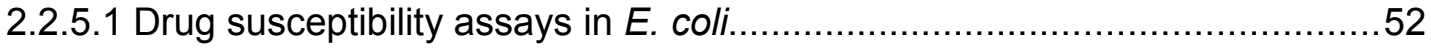

2.2.5.2 Sample preparation for Western-blot analysis........................................53

2.2.5.3 Dequalinium transport assay in whole cells.............................................54

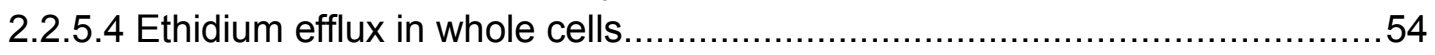

2.2.5.5 Norfloxacin accumulation assay in whole cells......................................55

2.2.5.6 Proton transport assay in inverted membrane vesicles............................55

2.2.5.7 Complementation of CraA to A. baumannii ATCC $19606 \Delta c r a A \ldots \ldots \ldots \ldots . . . . . .56$

2.2.5.8 Molecular docking of CraA ............................................................... 57

2.2.6 Characterization of TetA of $A$. baumannii strain AYE.......................................57

2.2.6.1 Drug susceptibility assays in E. coli................................................. 57

2.2.6.2 MIC determination of tetracyclines in $E$. coli overexpressing tetA or tetG....57

2.2.6.3 Tigecycline susceptibility assays in $A$. baumannii....................................58

2.2.6.4 RNA extraction and RT-qPCR ...................................................... 58

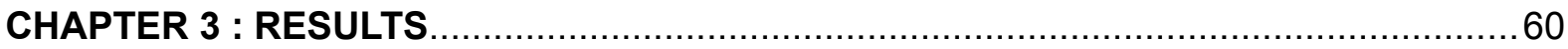

3.1 Construction of $A$. baumannii ATCC 19606 efflux pump knockout strains................60

3.2 Differential gene expression analysis of knockout strains...................................63

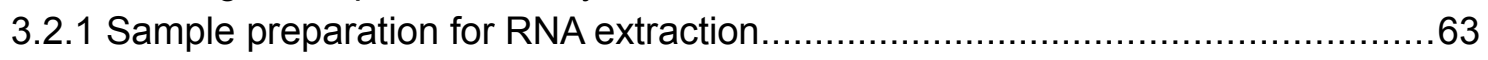

3.2.2 Quantitative reverse transcription PCR (RT-qPCR) .....................................64

3.2.2.1 Determination of PCR efficiency and melt curve analysis.........................64

3.2.2.2 Differential gene expression analysis.................................................66

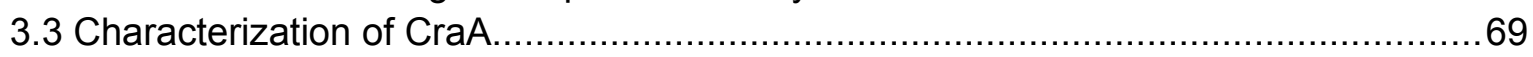

3.3.1 Putative chloramphenicol transporters in A. baumannii strain AYE....................69

3.3.2 Drug susceptibility assays of CraA variants in $E$. coli......................................71

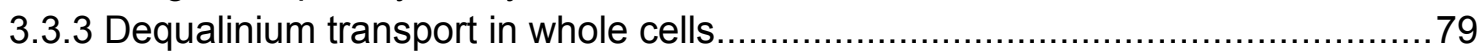

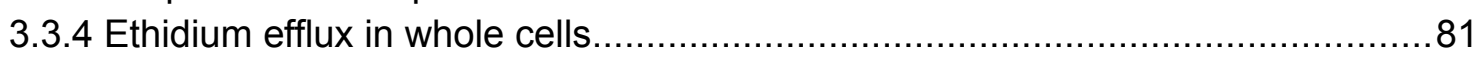

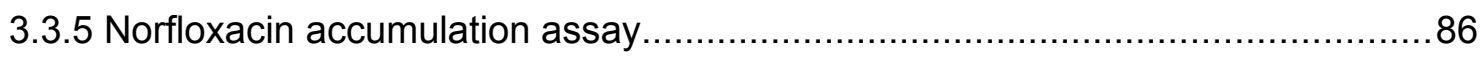

3.3.6 Proton transport assay using inverted membrane vesicles............................... 88

3.3.7 Phenotypic analysis of $A$. baumannii ATCC $19606 \triangle \mathrm{cra} A$ and complementation

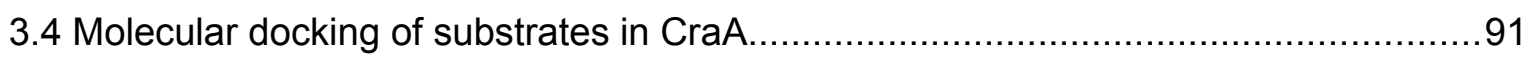

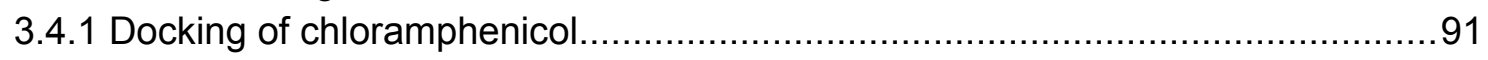

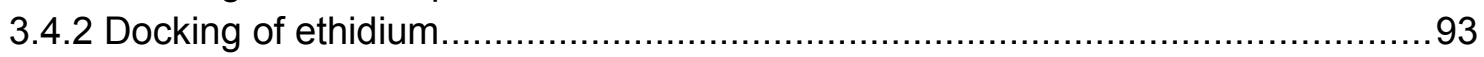

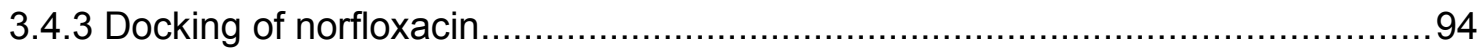

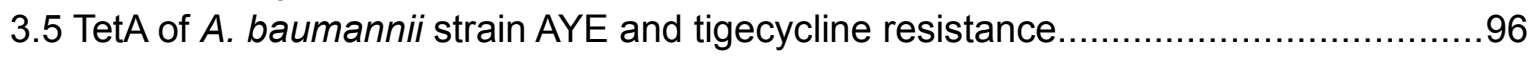

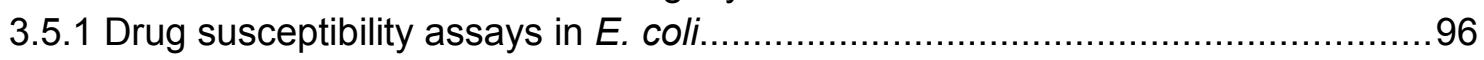

3.5.2 Drug susceptibility assays in $A$. baumannii............................................... 98

3.5.3 Differential gene expression analysis of tetA and RND efflux pump genes.........99

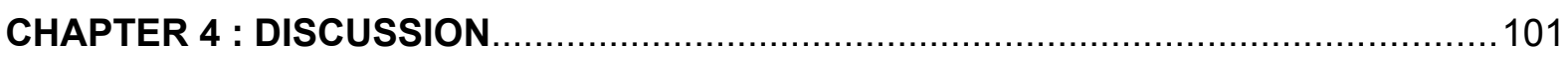

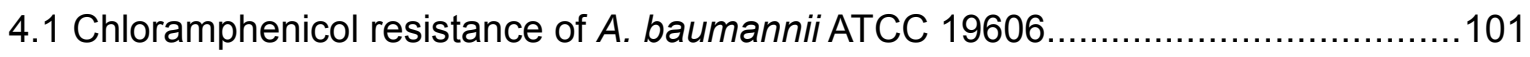

4.1.1 Differential gene expression of RND efflux pumps in $A$. baumannii.................103

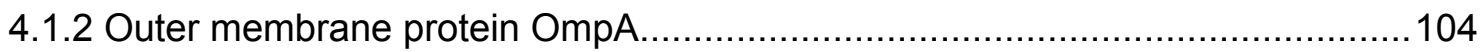

4.2 Chloramphenicol transporters and CraA of $A$. baumannii strain AYE.......................105

4.2.1 Insights into the functionally important residues of CraA ............................... 108

4.3 Tetracycline transporters of $A$. baumannii strain AYE.........................................111 
4.3.1 Efflux-based tigecycline resistance

4.3.2 Cooperation between single-component efflux pumps and RND tripartite systems

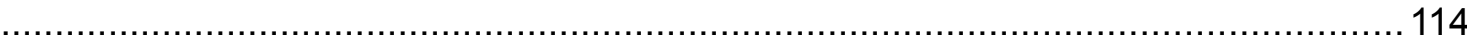

4.3.3 Mutations in TetA could lead to tigecycline resistance ..................................115

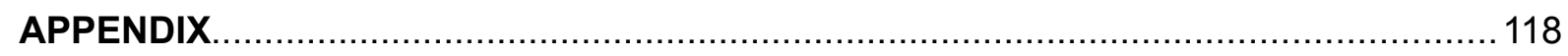

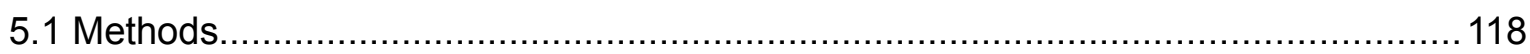

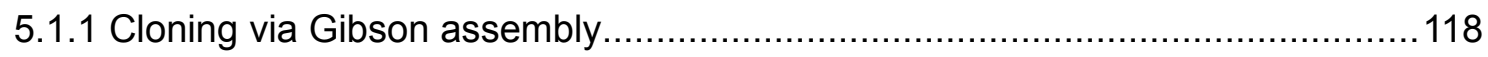

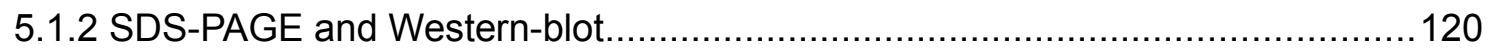

5.1.3 Preparation of chemically competent $E$. coli cells..................................... 121

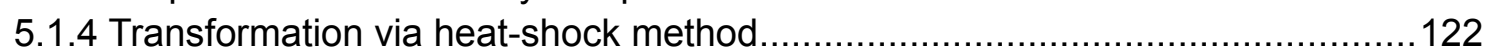

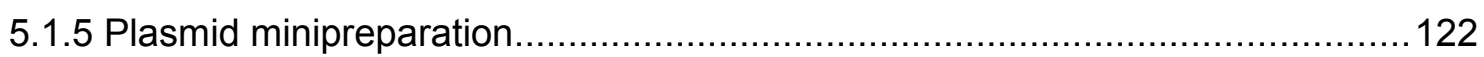

5.1.6 Preparation of electrocompetent A. baumannii ATCC 19606 cells...................122

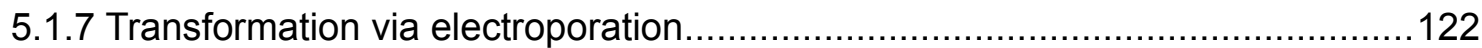

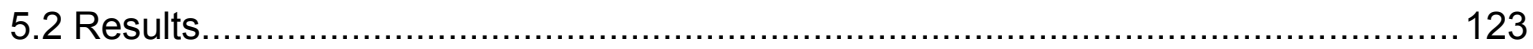

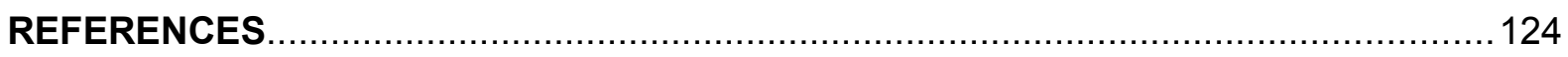

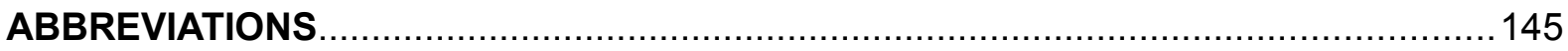

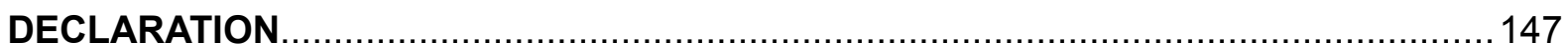

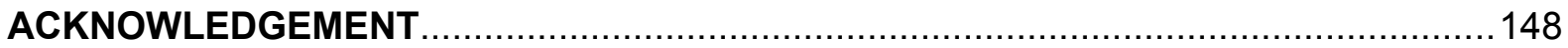

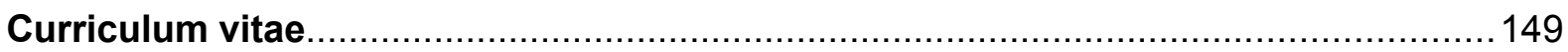




\section{Zusammenfassung}

Durch Acinetobacter baumannii verursachte nosokomiale Infektionen führen zu hohen Sterberaten, da sie nur schwer mit Antibiotika behandelt werden können. Aufgrund des dringenden Bedarfs an therapeutischen Alternativen priorisiert die WHO insbesondere die Erforschung der Carbapenem resistenten $A$. baumannii Stämme. Klinische $A$. baumannii Isolate werden als multiresistente Bakterien bezeichnet, da sie mehrere Resistenzmechanismen aufweisen. Eine der wichtigsten Komponenten, die zur Multiresistenz von $A$. baumannii beitragen, sind chromosomal kodierte oder erworbene Effluxpumpen. Die dreigliedrigen Systeme AdeABC, AdeEFG und AdelJK der ResistanceNodulation-Cell Division (RND) Familie befinden sich in der inneren und äußeren Membran der Bakterien und schleusen eine Vielzahl an toxischen Substanzen aus der Zelle. Zusätzlich tragen Einzelkomponenten-Effluxpumpen der Major Facilitator Superfamily (MFS), Multidrug and Toxin Extrusion (MATE), Small Multidrug Resistance (SMR) Familie und Proteobacterial Antimicrobial Compound Efflux (PACE) Familie zur spezifischen Resistenz von $A$. baumannii bei.

Eines der Hauptziele dieser Arbeit ist die Untersuchung, inwieweit die Deletion der Gene bestimmter Effluxpumpen in A. baumannii die Expression anderer (Effluxpumpen-) Gene beeinflusst, die für die Antibiotikaresistenz wichtig sind.

Die RND-Typ Effluxpumpen Deletionsstämme $\triangle a d e A B, \triangle a d e F G$ oder $\triangle a d e l J$ und der MFSTyp Chloramphenicol Effluxpumpen Deletionsstamm $\Delta c r a A$ wurden durch die Einführung des Suizidplasmids pBIISK_sacB/kanR zur homologen Rekombination in A. baumannii ATCC 19606 erzeugt. Zur Selektion wurde Sucrose verwendet. Die differentielle Genexpression der Knockout-Stämme wurde mittels RT-qPCR analysiert. Die Expression der Effluxpumpen Gene adeB, adeG, adeJ, craA und des Außenmembranproteins ompA wurde in Abwesenheit und in Gegenwart von Chloramphenicol untersucht. In Abwesenheit von Chloramphenicol konnte keine signifikante Veränderung des Genexpressionsniveaus in den Deletionsstämmen im Vergleich zum Wildtyp festgestellt werden. Dies weist darauf hin, dass die Deletion eines bestimmten Effluxpumpen Gens die Expression anderer Effluxpumpen Gene oder ompA in A. baumannii ATCC 19606 nicht beeinflusst hat. 
Allerdings wurde in Gegenwart von Chloramphenicol eine signifikante Zunahme des craA Expressionslevels, sowohl in Wildtyp- als auch in den Knockout-Stämmen (mit Ausnahme von $\triangle \operatorname{cra} A$ ), beobachtet.

Die Deletion des craA Gens im $\triangle c r a A$ Knockout $A$. baumannii Stamm führte zu einer 32fachen Abnahme des Minimale Hemm-Konzentration (MHK)-Werts von Chloramphenicol verglichen mit dem Wildtyp. Dies untermauert die Bedeutung der Effluxpumpe für die Chloramphenicolresitenz von A. baumannii. Im Gegensatz dazu, waren die MHK-Werte von Chloramphenicol für den A. baumannii $\Delta$ adelJ Stamm zwar ebenfalls wesentlich (achtfach) niedriger als die des Wildtyps, jedoch wurde das Expressionslevel von adeJ in Anwesenheit von Chloramphenicol nicht verändert. Selbst in Abwesenheit von Chloramphenicol war das adeJ-Transkript in hohen Mengen vorhanden, dies bestätigt, dass das AdelJK-System konstitutiv auf hohem Niveau exprimiert wird. Es wird postuliert, dass die AdelJKEffluxpumpe ein entscheidender Faktor für die Chloramphenicolresistenz ist und in Synergie mit CraA wirkt. Für die anderen RND-Effluxpumpen Gene adeB und adeG und das Außenmembranprotein Gen ompA wurden keine signifikanten Unterschiede im Expressionsniveau beobachtet, was darauf hinweist, dass diese Pumpen nicht wesentlich zur Chloramphenicolresistenz beitragen.

Des Weiteren gibt es einen in Frankreich epidemischen Stamm von A. baumannii, Stamm AYE, der im Gegensatz zum A. baumannii Stamm ATCC 19606 hochgradig multiresistent ist. Stamm AYE codiert eine große Resistenz-Insel, AbaR1, mit 45 Genen, die mit Antibiotika-, Antiseptika- und Schwermetallresistenzen assoziiert werden (Fournier et al., 2006). Fünf potentielle Chloramphenicoltransporter des A. baumannii Stammes AYE (CraA, CmIA, CmIA5, ABAYE0913 und ABAYE0685), die zur MFS gehören, wurden untersucht. Der Transportmechanismus von CraA, als einer der fünf Transporter, steht im Mittelpunkt dieser Arbeit, da er in klinischen Isolaten von $A$. baumannii weit verbreitet ist. CraA ist homolog zu dem gut untersuchten Effluxtransporter MdfA aus Escherichia coli $(61 \%$ Ähnlichkeit), fungiert jedoch überraschenderweise als spezifischer Chloramphenicoltransporter von A. baumannii (Roca et al., 2009). 
Während die Phenicolresistenz in Experimenten mit A. baumannii ATCC 19606 (unter Verwendung von $\triangle c r a A$ und Wildtyp Stämmen) bestätigt wurde, war die Substratspezifität von CraA in E. coli BW25113 $\triangle$ emrE $\Delta m d f A$ vielfältiger und ähnelte der des $E$. coli MdfA Homologs. CraA führte zur Resistenz der Zellen gegen Phenicole (Chloramphenicol, Thiamphenicol und Florfenicol), monovalente kationische Arzneimittel (Benzalkonium, TPP ${ }^{+}$ und Ethidium), langkettige dikationische Arzneimittel (Dequalinium und Chlorhexidin), Fluorchinolone (Norfloxacin und Ciprofoxacin) und Krebsmedikamente (Mitomycin C). Die Diskrepanz in der Substratspezifität kann durch das Vorhandensein anderer Effluxpumpen mit ähnlicher Substratspezifität und/oder die geringe Permeabilität der äußeren Membran von $A$. baumannii gegenüber diesen nicht-Phenicol-Arzneimitteln erklärt werden.

Um die molekularen Determinanten für die Bindung und den Transport von diversen Arzneimitteln zu untersuchen, wurden 45 Substitutionsvarianten von CraA, mit meist einzelnen Alanin Substitutionen, erstellt. Dabei wurden sowohl Reste substituiert, die für die Protonierung wichtig sind (E38, D46 und E338), als auch Reste, die für die Bindung und den Transport der Arzneimittel verantwortlich sind. Die funktionellen Reste für die Arzneimittelbindung und Transport wurden anhand von Docking-Experimenten auf der Basis eines von MdfA abgeleiteten CraA-Modells ermittelt. Abgesehen von Tests zur Arzneimittel Sensitivität wurden mit ausgewählten Varianten auch Transport- oder Akkumulationsstudien unter Verwendung der fluoreszierenden Substrate Ethidium, Dequalinium und Norfloxacin durchgeführt. Darüber hinaus wurde die Arzneimittel/ $\mathrm{H}^{+}$Antiportaktivität von CraA unter Verwendung von ACMA (9-amino-6-chloro-2methoxyacridine)-Fluoreszenzstudien in invertierten Membranvesikeln analysiert, die CraA oder CraA-Varianten enthielten.

Die Ergebnisse der verschiedenen Experimente zeigten einen hohen Grad an funktionellen Ähnlichkeiten zwischen MdfA und CraA. Die essenziellen protonierbaren Reste E26 und D34 (E38 und D46 in CraA) sind bei beiden Homologen für den Transport wichtig. Die CraA-Mutante E38A zeigt bei allen getesteten Arzneimitteln keine Transport-Aktivität, während die Mutante D46A nur bei manchen Arzneimitteln inaktiv ist. Dies deutet darauf hin, dass nur E38 am $\mathrm{H}^{+}$-Transport beteiligt ist. Auch der konservierte CraA-Rest R124 (R112 von MdfA) ist für die Aktivität der beiden Transporter essenziell. Basierend auf den 
nach innen und nach außen geöffneten Strukturen von MdfA wird vorgeschlagen, dass die R124-Y73-Kation-m-Wechselwirkung besonders wichtig ist, um die Integrität von CraA in seinem nach innen geöffnetem, verschlossenen oder nach außen geöffnetem Zustand aufrechtzuerhalten.

Die Alanin Substitution mehrerer Reste, die sich nicht in der potentiellen Bindungstasche befinden (Y139, M158, N283, Y346, R347), verringerte überraschenderweise die Resistenz von E. coli gegen Arzneimittel wie Norfloxacin, Ethidium, Florfenicol und/oder Chlorhexidin. Es wird vermutet, dass diese Reste, die sich in der Nähe der Furche befinden, welche ausgehend von der zytoplasmatischen Seite der Membranlipidebene zugewandt ist, an der Substrataufnahme beteiligt sind. Dies deutet darauf hin, dass einige der in die Membran eingebetteten Substrate möglicherweise eher aus der Innenseite der inneren Membran entnommen werden, als aus dem Zytoplasma.

Ein weiterer Fokus dieser Arbeit liegt auf der Untersuchung der drei Tetracyclintransporter $\operatorname{der}$ A. baumannii Stämme AYE; TetA, TetG und TetA(A). Die Transporter wurden in E. coli BW25113 $\Delta$ emrEAmdfA Zellen überexprimiert. Die Zellen wurden auf ihre Sensitivität gegenüber Tetracyclin, Minocyclin, Doxycyclin und dem Antibiotikum Tigecyclin, welches nur als letzte Instanz bei Behandlungen eingesetzt wird, untersucht. Die Überexpression von $\operatorname{tet} A(A)$ wies einen toxischen Effekt auf die Zellen auf, weshalb $\operatorname{Tet} A(A)$ nicht weiter untersucht wurde. Sowohl TetA als auch TetG führen zur Resistenz gegen Tetracyclin, Minocyclin und Doxycyclin. Entgegen der Erkenntnis, dass Tigecyclin von TetracyclinEffluxpumpen nicht erkannt wird, wurde der aktive Transport von Tigecyclin durch TetA festgestellt. Die Rolle von TetA im Tigecyclintransport von $A$. baumannii Stamm ATCC 19606 wurde durch die Untersuchung der Tigecyclin-Sensitivität von Zellen ohne tetA Gen mit und ohne TetA Komplement bestätigt.

Es wird vermutet, dass TetA, welches sich in der inneren Membran befindet, in Zusammenarbeit mit dreigliedrigen Systemen der RND-Familie wirkt. Diese sind in der inneren und äußeren Membran lokalisiert, um Tigecyclin über die äußere Membran aus dem Periplasma zu transportieren. Der A. baumannii ATCC $19606 \Delta a d e A B$ Stamm war im Gegensatz zu dem Wildtyp sensitiv gegenüber Tigecyclin. Die Deletion von adelJ führte 
ebenfalls zu einer erhöhten Sensitivität der Zellen gegenüber Tigecyclin, jedoch im geringeren Ausmaß verglichen mit dem $\triangle a d e A B$-Phänotyp. Für A. baumannii ATCC 19606 $\triangle$ adeFG konnte keine Veränderung der Tigecyclin Sensitivität festgestellt werden. Differentielle Genexpressionsanalysen der RND-Effluxpumpen Gene (adeB, adeG und adeJ) und tetA des $A$. baumannii Stamms AYE zeigten, dass die Expression von tet $A$ signifikant erhöht ist, wenn Tigecyclin im Wachstumsmedium vorhanden ist. Tigecyclin hatte auf die Expression von adeB und adeJ keinen Einfluss. Die adeG-Expression war jedoch in Gegenwart von Tigecyclin erhöht, obwohl die AdeFGH-Pumpe scheinbar nicht zur Tigecyclinresistenz beiträgt.

Zusammenfassend zeigt diese Arbeit, dass die Deletion einer einzelnen Effluxpumpe in $A$. baumannii ATCC 19606 keinen signifikanten Einfluss auf die Expression anderer Effluxpumpen Gene hat. Kreuzregulierung zwischen Effluxpumpen konnte nicht beobachtet werden. Die Expression der wichtigsten RND-Systeme von A. baumannii war relativ stabil und Chloramphenicol hatte keinen Einfluss auf das Expressionsniveau. CraA in $A$. baumannii ATCC 19606 wurde als die wichtigste Determinante der Chloramphenicolresistenz bestätigt. CraA galt bisher als spezifischer ChloramphenicolTransporter, allerdings lieferten detaillierte in vivo und in vitro Transportstudien wesentliche Beweise dafür, dass CraA tatsächlich ein Transporter für mehrere Arzneimittel ist. Durch gezielte Mutagenese und phänotypische Charakterisierung der CraA-Varianten wurde die strukturelle und funktionelle Homologie zwischen dem E. coli Multidrug-Transporter MdfA und CraA gezeigt. Die Charakterisierung von drei Tetracyclintransportern des A. baumannii Stammes AYE zeigte, dass TetA am Transport von Tigecyclin beteiligt ist und, dass der Phänotyp der Tigecyclinresistenz in Synergie mit den Effluxsystemen des RND-Typ auftritt. Tigecyclin stimuliert die Produktion der Einkomponenten-Effluxpumpe TetA. TetA transportiert Tigecyclin aus dem Zytoplasma in das Periplasma, von wo aus es durch die konstitutiv vorhandenen dreigliedrigen RND-Systeme AdeABC und AdelJK weiter über die Außenmembran transportiert wird. Diese Erkenntnisse verdeutlichen die entscheidende Rolle der MFS-Transporter CraA und TetA für die potenzielle Gefahr einer erfolglosen Behandlung von Infektionen mit $A$. baumannii. Daher sollte die erhöhte Produktion dieser Effluxpumpen in den klinischen Isolaten von $A$. baumannii engmaschig überwacht werden. 


\section{Abstract}

Acinetobacter baumannii is a worldwide opportunistic pathogen responsible for nosocomial infections. One of the main factors contributing to multidrug resistance in $A$. baumannii is the upregulation of various chromosomally encoded or acquired efflux pumps, which expel toxic compounds out of the cells with high efficiency. One of the major objectives for this thesis is to investigate whether the deletion of a certain efflux pump genes in $A$. baumannii would affect the expression of other (efflux pump) genes which are important in conferring antibiotic resistance. The resistance-nodulation-cell division (RND)-type efflux pump gene deletion strains $\triangle a d e A B, \triangle a d e F G$ or $\triangle a d e l J$ and the major facilitator superfamily (MFS) chloramphenicol efflux pump gene deletion strain $\Delta$ craA of $A$. baumannii ATCC 19606 were created, followed by a differential gene expression study via RT-qPCR. The expression of efflux pump genes ade $B$, adeG, adeJ, $c r a A$, and the outer membrane protein ompA were examined in the absence and presence of chloramphenicol.

No significant up- or downregulation of these genes for any of these deletion strains in comparision to the wild-type strain in absence of the drug chloramphenicol. This indicates that deletion of certain efflux pump did not alter the expression of other efflux pumps or ompA in $A$. baumannii ATCC 19606. In contrast, cra $A$ was significantly upregulated in $A$. baumannii exposed to chloramphenicol, emphasizing the importance of CraA in chloramphenicol resistance. CraA is widely present in clinical isolates of $A$. baumannii. It is homologous to the well-studied multiple-drug efflux transporter MdfA from Escherichia coli (61\% similarity), but surprisingly reported to be acting as a specific chloramphenicol transporter of $A$. baumannii (Roca et al., 2009).

The drug susceptibility assay done with $A$. baumannii ATCC $19606 \Delta c r a A$ showed that CraA could confer resistance towards phenicols (chloramphenicol, thiamphenicol, and florfenicol), which was in line with the previous report. CraA was heterologously overproduced in $E$. coli BW25113 $\Delta$ emrEAmdfA and its substrate specificity was determined by drug susceptibility assays and whole cell fluorescent dye uptake experiments. We observed that the substrate specificity of $c r a A$ overexpressed in $E$. coli was more diverse and resembling that of the $E$. coli MdfA homolog. Apart from resistance towards phenicols (chloramphenicol, thiamphenicol, and florfenicol), CraA also confer resistance towards monovalent cationic 
drugs (benzalkonium, $\mathrm{TPP}^{+}$, and ethidium), long dicationic drugs (dequalinium and chlorhexidine), fluoroquinolones (norfloxacin and ciprofoxacin) and anticancer drugs (mitomycin $\mathrm{C}$ ). We showed that $\mathrm{CraA}$ is a drug/ $\mathrm{H}^{+}$antiporter by $\mathrm{ACMA}$ quenching in inverted CraA or CraA variant containing membrane vesicles.

To address the molecular determinants for multidrug binding and transport, 45 mostly single Ala-substitution variants of CraA were created. These include substitution variants for membrane-embedded proton-titratable residues (E38, D46, and E338) and residues predicted to be important for binding and transport of drug, as inferred from docking experiments on basis of a MdfA-derived CraA model. The combined results indicated a high degree of functional similarities between MdfA and CraA. The conserved titratable residues E26 and D34 (E38 and D46 in CraA) are important for transport in both these homologs. The CraA variant E38A is inactive against all tested drugs, but D46A is only inactive for some drugs, suggesting that only E38 is involved in $\mathrm{H}^{+}$-transport.

Ala-substitution of several residues not located in the putative binding pocket (Y139, M158, N283, Y346, R347) surprisingly reduced the resistance of E. coli against drugs such as norfloxacin, ethidium, florfenicol and/or chlorhexidine. These residues which are located in proximity to the cleft facing the membrane lipid plane from the cytoplasmic side were postulated to be involved in substrate uptake, suggested that some of the membraneembedded substrates might be taken up from the inner leaflet of the inner membrane rather than from the cytoplasm.

Another focus of this thesis is the three tetracycline transporters of $A$. baumannii strain AYE: TetA, TetG and TetA(A). Susceptibility assays involving tetracycline, minocycline, doxycycline and the last-resort antibiotic tigecycline were conducted on E. coli BW25113 $\Delta$ emrE $\Delta m d f A$ overexpressing these transporters. $\operatorname{Tet} A(A)$ was excluded from further study due to toxicity of the cells caused by protein overexpression. Both TetA and TetG confer resistance against tetracycline, minocycline and doxycycline. Although tigecycline was reported not to be recognized by tetracycline efflux pumps, we surprisingly found that TetA is able to transport tigecycline. The role of TetA in tigecycline efflux in $A$. baumannii was confirmed by conducting tigecycline susceptibility assays on $A$. baumannii. 
We speculate that TetA embedded in the inner membrane acts in cooperation with RND-type tripartite systems that span the inner and outer membrane to extrude tigecycline from the periplasm across the outer membrane. A. baumannii ATCC $19606 \triangle$ adeAB were indeed sensitive to tigecycline in comparison to wild-type strain. Deletion of adelJ also leads to sensitivity to tigecycline, but less so compared to the $\triangle a d e A B$ phenotype, while $A$. baumannii ATCC $19606 \triangle$ adeFG did not show any difference compared to wild-type strain in tigecycline susceptibility. Differential gene expression analysis of the RND efflux pumps (adeB, adeG and adeJ) and tetA of $A$. baumannii strain AYE showed that the expression of tet $A$ expression is significantly upregulated when tigecycline is present in the growth medium.

We conclude that craA encodes a broad-spectrum efflux pump rather than a specific chloramphenicol transporter. In $A$. baumannii, the synergistic effects with the outer membrane and/or the presence of other transporters could result in the discrepancy observed. Thus, the possibility of CraA in conferring multidrug resistance should not be overlooked, especially when it is up-regulated under antibiotic stress conditions. 


\section{CHAPTER 1: INTRODUCTION}

\subsection{Antibiotics - Modes of action and resistance mechanisms}

Before the large-scale production of penicillin in 1940s, infectious diseases such as the pneumonia, tuberculosis, and diarrhea were the leading cause of mortality (CDC on Infectious Diseases in the United States: 1900-99). The period between 1950s and 1970s was the golden era of novel antibiotics, with discovery of more than 20 classes of antibiotics, particularly from the soil bacteria Streptomyces (Durand et al., 2019). Most antibiotics target cellular processes such as DNA replication, transcription, translation, or cell wall synthesis (Chellat et al., 2016, Figure 1.1).

Since the first antibiotic penicillin discovered by Alexander Flemings in 1928, $\beta$-lactams are still the most commonly prescribed drug classes, with over $60 \%$ of all antibiotics currently used for bacterial infection treatment in humans. This includes the next generation $\beta$-lactams cephalosporins, carbapenems and monobactams. The binding of $\beta$-lactams to the penicillinbinding proteins interrupts the peptidoglycan synthesis and disrupts the cell wall formation (Ribeiro da Cunha et al., 2019). Polymyxins (e.g. colistin) kill the bacteria through membrane lysis. The binding of positively charged polymyxin to lipopolysaccharide (LPS) destabilizes the lipid bilayer and increases the membrane permeability, finally lead to leakage of the cytoplasmic content and cell death (Poirel et al., 2017).

Protein synthesis is an important cellular target of several classes of antibiotics. Macrolides (e.g. eyrthromycin), oxazolidinones (e.g. linezolid) and chloramphenicol target the large 50S subunit, while aminoglycosides (e.g. kanamycin) and tetracyclines interfere with the smaller 30 S subunit (Chellat et al., 2016).

Some antibiotics for example the rifamycins (e.g. rifampicin) inhibit the transcription process. Their high affinity for prokaryotic RNA polymerase interrupts the RNA synthesis of the cells (Calvori et al., 1965). Fluoroquinolones (e.g. norfloxacin, ciprofloxacin) inhibit DNA synthesis by binding to gyrase. Another target for antibiotics such as sulfonamides and trimethoprim is 
the folate synthesis. It relates to many pathways that allow the correct synthesis of proteins and nucleic acids (Fernández-Villa et al., 2019).

Unfortunately, gene transfer of multiple resistance determinants or mutations (Figure 1.1) occurs promptly under selective pressure arising from overuse and misuse of antibiotics. Emergence of multidrug resistance has become a threatening scenario for public health worldwide. Bacteria can enzymatically degrade or modify the antibiotics, for example through the production of $\beta$-lactamases that hydrolyze the $\beta$-lactam ring and deactivate the $\beta$-lactam antibiotic. Another mechanism is the modification of the antibiotic target site. For example, the action of rifampicin is inhibited by the mutations in the rpoB gene that encodes the $\beta$ subunit of RNA polymerase. The cells also protect themselves by altering the membrane permeability to decrease the penetration of antibiotics, or actively extruding the antimicrobial compounds through efflux pumps (Chellat et al., 2016, Figure 1.1).

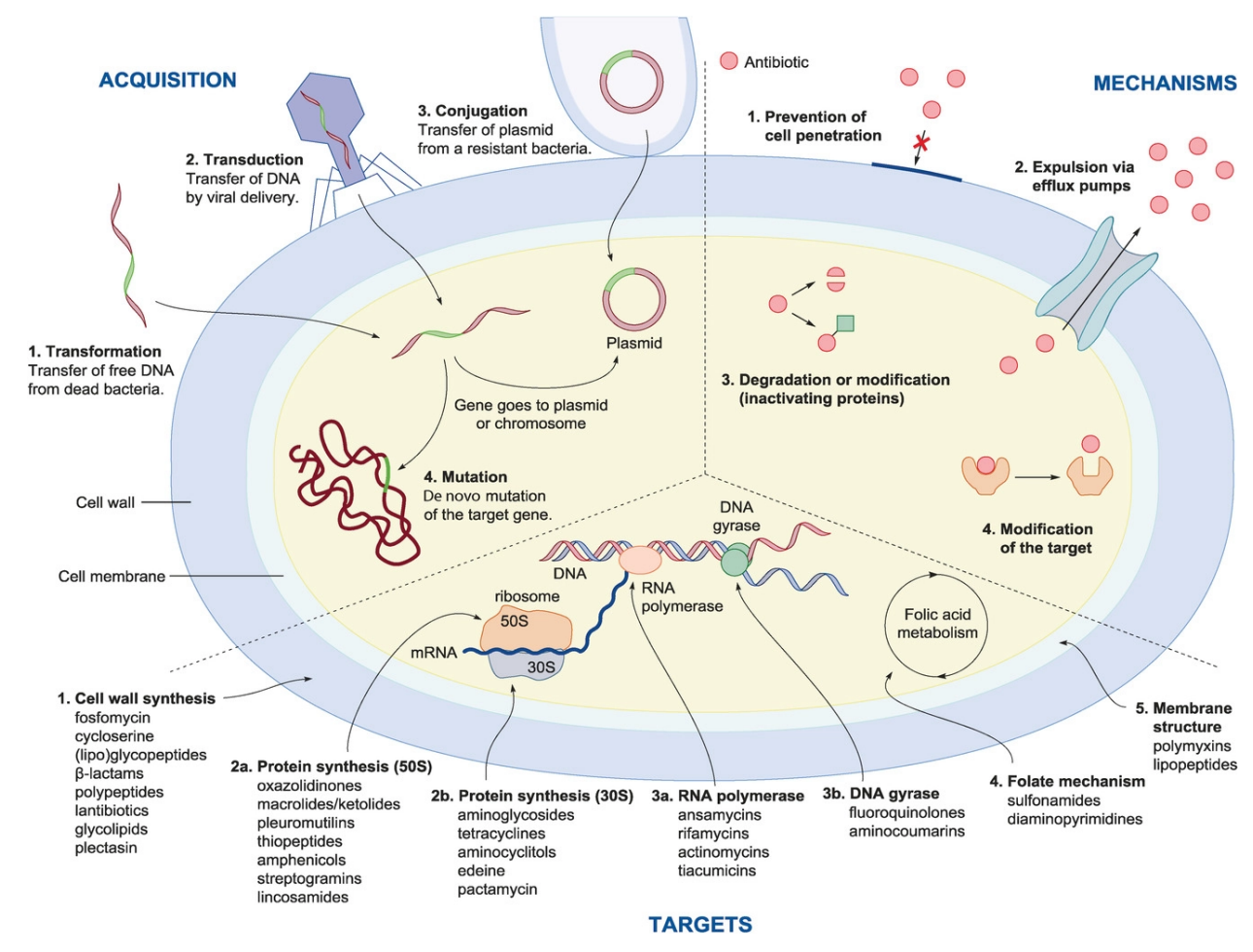

Figure 1.1 The main acquisition pathways, resistance mechanisms, and main targets for antibiotics (adopted from Chellat et al., 2016) 


\subsection{Classes of multidrug efflux pumps}

The intrinsic resistance of Gram-negative bacteria to many different drugs is largely attributed to the architecture of the cell envelope which consists of double membrane. The asymmetric outer membrane with lipopolysaccharide (LPS) bound on the outer leaflet is an extra barrier for antibiotics to access their targets. Small hydrophobic drugs generally diffuse through the lipid bilayer, while hydrophilic drugs pass through the porin channels. Some antibiotics such as vancomycin are unable to penetrate the outer membrane (Nikaido, 1989 ; Nikaido, 1996).

The presence of efflux pumps embedded in the inner membrane also contributes to multidrug resistance. The efflux pumps are classified into several superfamilies based on primary amino acid sequence phylogeny, including the ATP-binding cassette (ABC) superfamily, resistance-nodulation-cell division (RND) superfamily, major facilitator superfamily (MFS), multidrug and toxin extrusion (MATE) family, and small multidrug resistance (SMR) family (Poole, 2005 ; Du et al., 2018, Figure 1.2). A recently identified class is the proteobacterial antimicrobial compound efflux (PACE) family (Hassan et al., 2013).

Members of the ABC superfamily are primary active transporters that uses ATP as energy source. The other superfamilies are secondary active antiporters that use the energy provided by the electrochemical gradient across the inner membrane. As the efflux pumps exist before the era of antibiotics, it is postulated that the physiological functions of multidrug efflux pumps include the secretion of intracellular metabolites and protection against a variety of substances in the host environment such as bile salts and fatty acids (Paulsen et al., 1996 ; Poole, 2005 ; Piddock, 2006). 


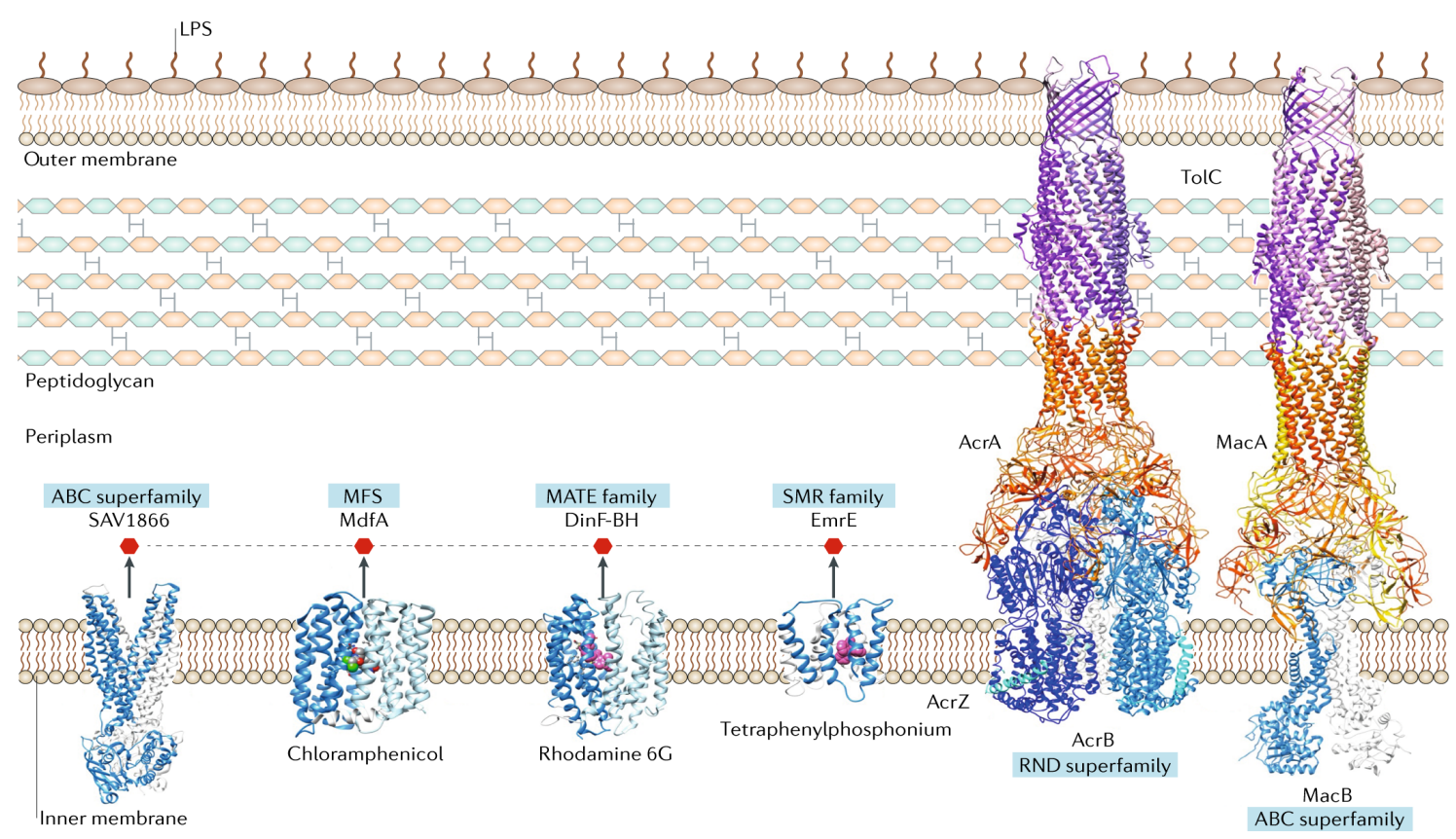

Figure 1.2 Structures of representatives from the five families of the multidrug efflux pumps (adopted from Du et al., 2018).

\subsubsection{RND superfamily}

RND transporters are ubiquitously found in all domains of life (Tseng et al., 1999). Bacterial multidrug efflux RND transporters are embedded in the inner membrane and are catalytically active in conjunction with a periplasmic membrane fusion protein (MFP) and an outer membrane factor (OMF) as a tripartite efflux system (Nikaido, 1996). By far the best characterized RND protein is AcrB from E. coli. Together with AcrA and TolC, the tripartite complex is able to extrude antibiotics (such as macrolides, fluoroquinolones, beta-lactams, tetracyclines, chloramphenicol), dyes (such as acriflavin, ethidium), detergents (such as SDS, bile salts, Triton X-100) and solvents (such as hexane, cyclohexane, octane) out of the cells (Pos, 2009). AcrB consists of a transmembrane domain with 12 TM helices and a large periplasmic domain. TolC is a pore-like outer membrane-embedded protein comprising a 40 $\AA \beta$-barrel that spans the outer membrane and $100 \AA \alpha$-barrel protruding into the periplasm. The a-helical hairpin of AcrA forms tip-to-tip interaction with the a-barrel tip region of ToIC (Wang et al., 2017). TolC shared by other transporter systems besides AcrAB-TolC, and it 
has little or no function in determining the substrate specificity or directionality of transport (Koronakis et al., 2000).

AcrB is active as a homotrimer. Although the protomers in AcrB trimer are symmetric in the resting state (Murakami et al., 2002), it adopts three principle states upon drug binding, the loose $(\mathrm{L})$, tight $(\mathrm{T})$ and open $(\mathrm{O})$ state. Every protomer can adopt any of these states and within the trimer a protomer interdependent functional rotation occurs (Seeger et al., 2006 ; Murakami et al., 2006). The functional rotation cycle starts with the loose binding of substrate at a low affinity site on the $L$ protomer, followed by conformational change to the $T$ protomer with tight binding of the substrate in the designated deep binding pocket and finally conversion to the $\mathrm{O}$ protomer resulting in the release of the substrate towards TolC. Conversion from the $\mathrm{T}$ state to the $\mathrm{O}$ state requires the binding of a proton to the proton translocation site. The release of a proton from the proton translocation site to the cytoplasm converts the $O$ protomer to the $L$ state restarting the cycle (Seeger et al., 2006, Figure 1.3).

A

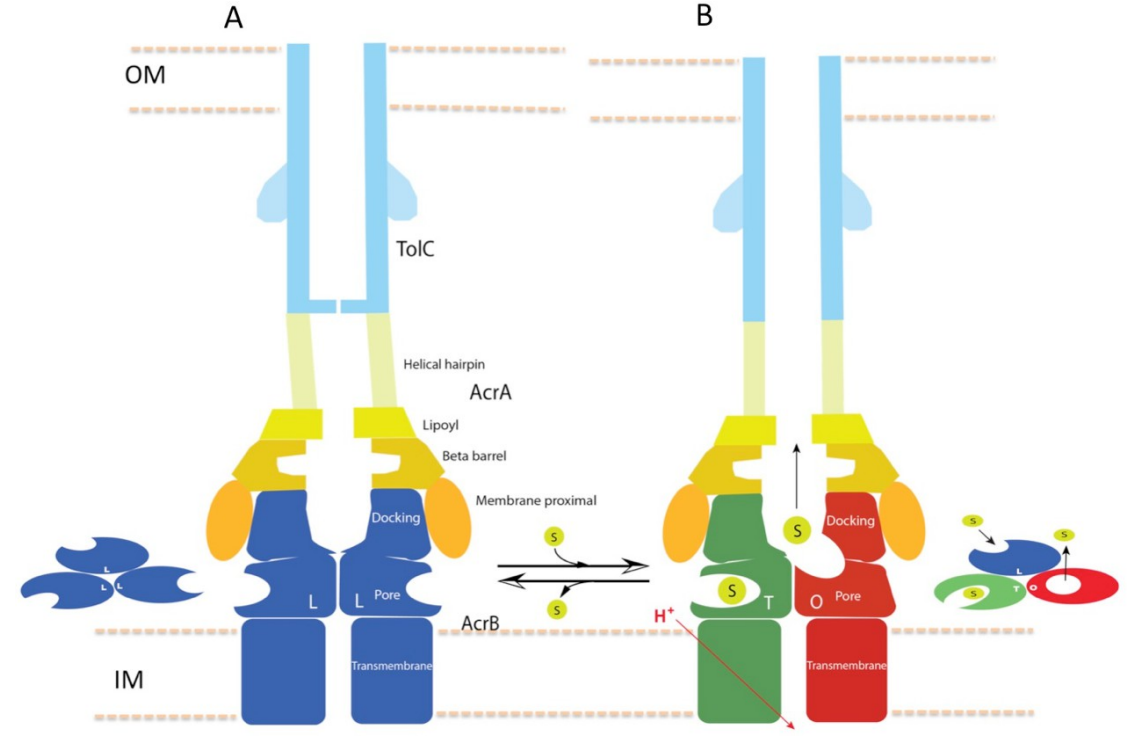

Figure 1.3 Schematic representation of the AcrB functional rotation transport mechanism. The conformation $L$, $\mathrm{T}$, and $\mathrm{O}$ are coloured blue, green and red, respectively. (A) The resting state of the apo pump with TolC in closed-state and the AcrB trimer in LLL conformation. (B) In the presence of substrates, the apo pump switches to a transport-state, opening the TolC channel. Each AcrB protomer cycles through L, T and O states, driven by the proton motive force (red arrow). In the absence of substrate, the pump reverts to the resting state and closes the TolC channel. IM=inner membrane, OM=outer membrane (adopted from Wang et al., 2017). 


\subsubsection{ABC transporter}

ABC transporters are also found in all domains of life (Higgins, 1992). They function either as importers or exporters, with the importers present in prokaryotes and plants, and the exporters present in all domains of life. The members of this superfamily are characterized by two nucleotide-binding domains (NBDs) and two transmembrane domains (TMDs). The highly conserved NBDs bind and hydrolyze ATP, providing energy for substrate translocation across the cell membrane by the TMDs. The TMDs are highly diverse, indicate the large substrate diversity of this transporter superfamily. In bacteria and archaea, the four domains are often distinct subunits, or they are fused into homo- or heterodimerizing half-transporters composed of one NBD and one TMD. In eukaryotes, ABC transporters are typically constituted by a single polypeptide that contains all four domains (e.g. P-glycoprotein). ABC importers require an additional substrate-binding protein (SBP) that binds the substrate and delivers it to the binding site in the TMDs (Locher, 2009). ABC transporters can be divided into seven types based on their TMD fold. ABC transporters of types I to III are importers, types IV and V are primarily exporters, type VI and type VII are mechanotransducers (Thomas and Tampé, 2020 ; Greene et al., 2018, Figure 1.4).

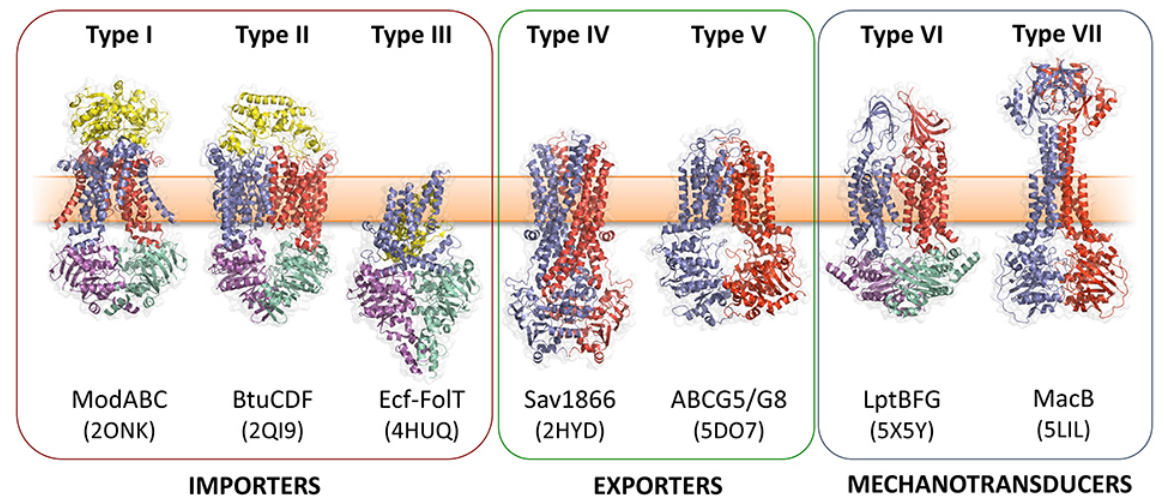

Figure 1.4 Structures of representatives of seven types of $A B C$ transporters.

The members of $A B C$ superfamily which act as drug exporters exist as single-component transporters (e.g. SAV1866 of Staphylococcus aureus) or tripartite complexes such as the MacAB-TolC complex of E. coli (Kobayashi et al., 2001). MacAB-TolC drives not only the efflux of macrolide antibiotics but also the transport of outer membrane lipopeptides, 
protoporphyrin, polypeptide virulence factors and lipopolysaccharides (Greene et al., 2018). Beside stabilizing the tripartite assembly, MacA also has a role in regulating the function of $\mathrm{MacB}$, as it promotes and stabilizes the ATP-binding form of the MacB transporter (Lin et al., 2009a). The cryo-EM structure reveals a hexamer of MacA bridging the TolC trimer in the outer membrane and the MacB dimer in the inner membrane, generating a quaternary structure with a central channel for substrate translocation. A gating ring found in MacA may act as a one-way valve in substrate transport (Fitzpatrick et al., 2017, Figure 1.5).

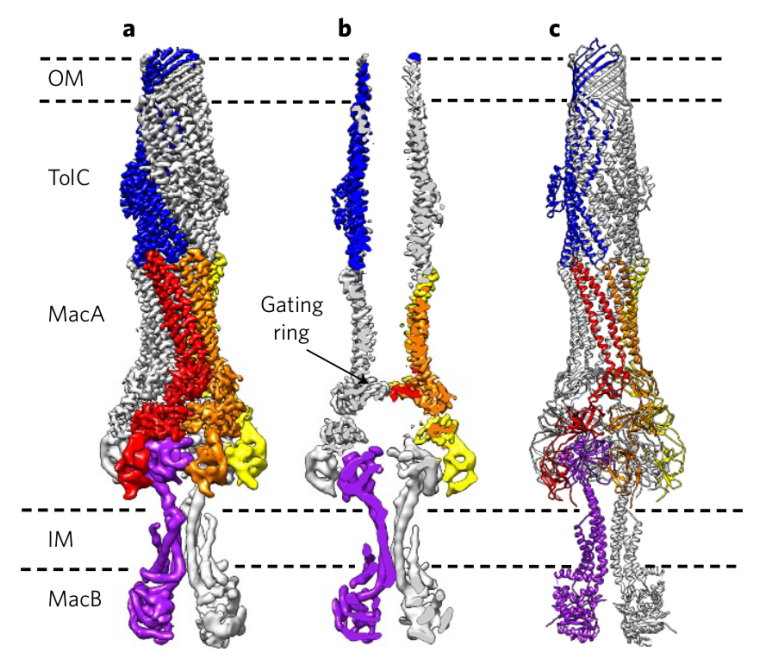

Figure 1.5 Pseudo-atomic model for the MacAB-TolC pump. (a) Five protomers are colour-coded: TolC (blue), MacA (red, orange and yellow) and MacB (purple). (b) Sliced view of the pump. (c) Model based on docked crystal structures. IM: inner membrane; OM: outer membrane.

\subsubsection{Major Facilitator Superfamily}

The Major Facilitator Superfamily (MFS) is the largest and most diverse superfamily of transporters found in all domains of life. Composed of at least 87 families, MFS transporters include uniporters, symporters and antiporters (Reddy et al., 2012). Uniporters transport single substrate down their concentration gradient and require no external energy input. Symporters and antiporters utilize the energy stored in the concentration gradient of their coupling ion or co-substrate. Symporters translocate substrates and coupling ion in the same direction simultaneously, while antiporters in opposite directions across the membrane (Yan, 2015). 
Among the drug exporters, single-drug or multidrug transporters are classified into three drug $/ \mathrm{H}^{+}$antiporter (DHA) families (DHA1, DHA2, DHA3), characterized on basis of the number of TM helices and the variation of motif $A$. Motif $A, G X_{3}-(D / E)-(R / K)-X-G-[X]-(R / K)-$ $(R / K)$ (where ' $X$ ' indicates any amino acid), located in the cytoplasmic loop between TM2 and TM3 (location shown in Figure 1.9) is the most conserved motif in MFS transporters (Paulsen et al., 1996). The members of DHA1 and DHA3 family possess a uniform topology of 12 TM helices organized as two domains, each composed of six helices (Chitsaz and Brown, 2017). Both $\mathrm{N}$ - and C-termini are located in the cytoplasm. The N-terminal half (TM1TM6) displays weak sequence homology to the C-terminal half (TM7-TM12), suggesting that the MFS proteins may have evolved from a gene duplication or fusion event (Nair et al., 2016). The members of DHA2 family, for example the QacA and QacB of Staphylococcus aureus has 14 TM helices (Paulsen et al., 1996b).

Most members of this superfamily function as monomers. The substrate translocation is carried out through an alternating access mechanism. This mechanism was proposed by Jardetzky in 1966 to describe the conformational change of a transport protein that alternate between an extracellular- and an intracellular-facing state in which the centrally located substrate binding site is accessible to only one side of the membrane at a time (Jardetzky, 1966).

A rocker-switch model of alternating access that involves conformational changes of the two halves of the protein among inward-open, occluded and outward-open states was thought to be the mechanism for most MFS transporters (Figure 1.6a). An elevator mechanism (Figure 1.6b) was later proposed for glutamate transporter homolog $G \mathrm{t}_{\mathrm{Ph}}$, in which the 'transport domain' containing undergoes a vertical movement to deliver substrate to the inside of the cell, while the 'scaffold domain' that anchors the protein in the membrane remains relatively static (Reyes at al., 2009 ; Ryan and Vandenberg, 2016). 


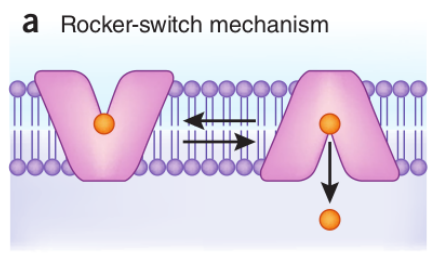

b Elevator mechanism

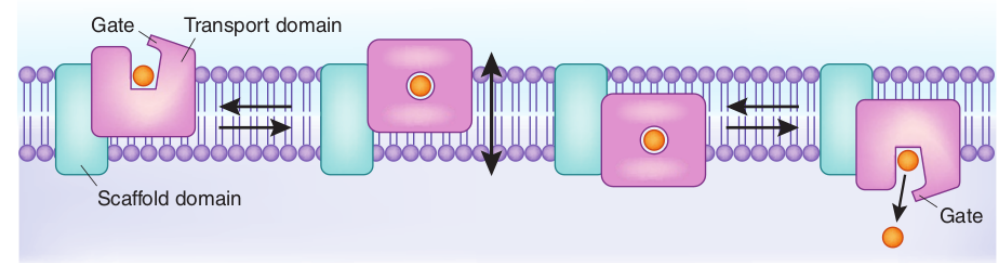

Figure 1.6 Alternative access mechanism of substrate across the membrane. a) Rocker-switch mechanism: the transporter must switch between an outward-facing conformation and an inward-facing conformation, in order to allow alternating access to the substrate binding site from either side of the membrane. b) Elevator mechanism: substrate is picked up on one side of the membrane, and a 'gate' subsequently closes. The transport domain undergoes a vertical translation relative to the membrane plane to carry the cargo across the membrane, another gate must then open to release the substrate (adopted from Ryan and Vandenberg, 2016).

\subsubsection{MdfA of E. coli}

MdfA of $E$. coli belongs to the DHA1 family within the MFS. This multidrug efflux pump comprising 410 amino acid residues confers resistance against a broad variety of structurally dissimilar compounds, including electroneutral drugs, mono- or divalent cations, and zwitterions. Apart from conferring antibiotic resistance, the ability of $m d f A$-expressing cells to survive under extreme alkaline $\mathrm{pH}$ conditions $(\mathrm{pH}>9)$ suggests that $\mathrm{pH}$ homeostasis is one of the physiological roles of this transporter (Lewinson et al., 2004).

The transport of neutral substrates by MdfA is electrogenic and driven by both components of the proton electrochemical gradient, the proton concentration gradient $(\Delta \mathrm{pH})$ and the membrane potential $(\Delta \Psi)$, while the transport of monovalent cationic substrates is electroneutral and therefore driven only by the $\Delta \mathrm{pH}$ (Lewinson et al., 2003 ; Fluman and Bibi, 2009, Figure 1.7). Thus, the drug/ $\mathrm{H}^{+}$stoichiometry for MdfA is identified as 1 in each transport cycle. The protons and substrates do not share a common binding site and the competition between them is possibly through an allosteric effect. The indirect competition mechanism enable more flexibility regarding the charge of its substrates (Fluman et al., 2012 ; Yardeni et al., 2018). 


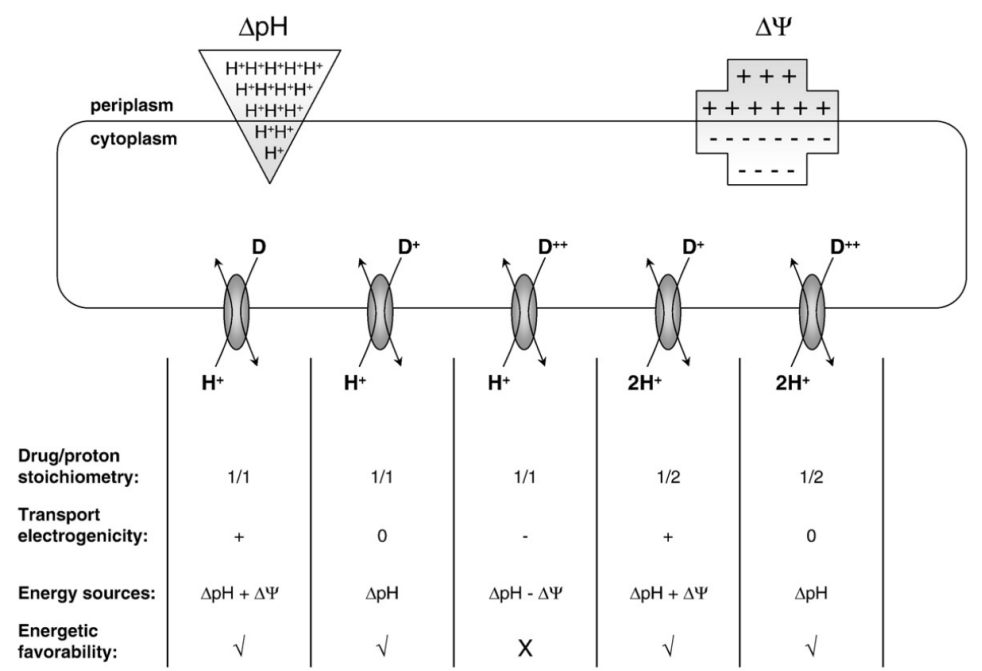

Figure 1.7 Bioenergetics of transport reactions of MFS efflux pump. The upper panel shows the two components of the proton-motive-force, $\Delta \mathrm{pH}$ and $\Delta \Psi$. The bioenergetics of transport for reactions with different drug $/ \mathrm{H}^{+}$stoichiometries are shown in the panel below. A positive electrogenicity refers to the import of a net positive charge into the cell. Import of a net negative charge against the gradient is energetically not favourable (adopted from Fluman and Bibi, 2009).

MdfA contains three membrane-embedded charged residues (Glu26, Asp34 and Arg112). The two conserved proton-titratable residues, Glu26 and Asp34 located on TM1, are important for the active transport of substrates. It was postulated that the transport mechanism involves competition between proton and substrate binding at these two acidic residues in the binding cavity of MdfA. Interestingly, neither Glu26 nor Asp34 is essential for drug binding, as many other determinants in the putative binding pocket are involved (Adler et al., 2004 ; Sigal et al., 2006 ; Sigal et al., 2009). Proton translocation in MdfA does not require a precise architecture at the proton binding site, it tolerates the displacement of an essential negative charge to various positions inside the putative drug translocation pathway in the protein (Sigal et al., 2009).

It was a breakthrough in the study of MdfA, when high resolution crystal structures were reported by Heng et al. in 2015. Three substrate-bound structures of a Q131R MdfA variant showed an inward-open conformation, with drug bound near the conserved acidic residue Asp34 (Heng et al., 2015, Figure 1.8a). It was proposed that Glu26 gets protonated in the outward-facing conformation and then the proton moves to Asp34. Binding of an electroneutral substrate increases the positive electrostatic field generated by Arg112 of motif $B$ which subsequently triggers the deprotonation of Asp34 (Figure 1.8b). Motif B 
("RxxQG") in TM4 (location shown in Figure 1.9) is the only high-frequency site that contains a membrane-embedded basic residue among MFS members. Arg112 is the most evolutionarily conserved residue in MdfA orthologs. It was shown that a positive charge at this position is essential for the function of MdfA (Sigal et al., 2005 ; Heng et al., 2015).

a)
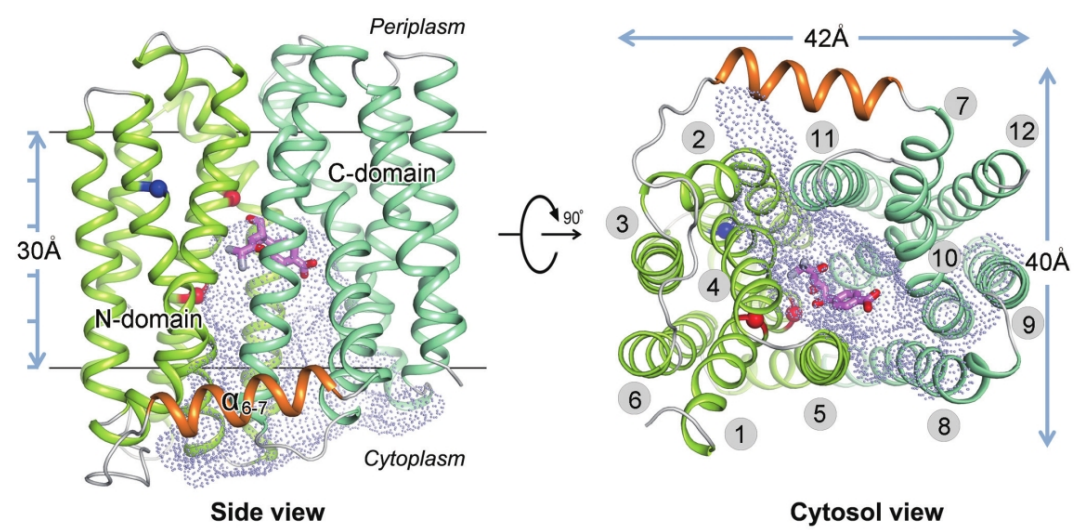

Cytosol view

b)

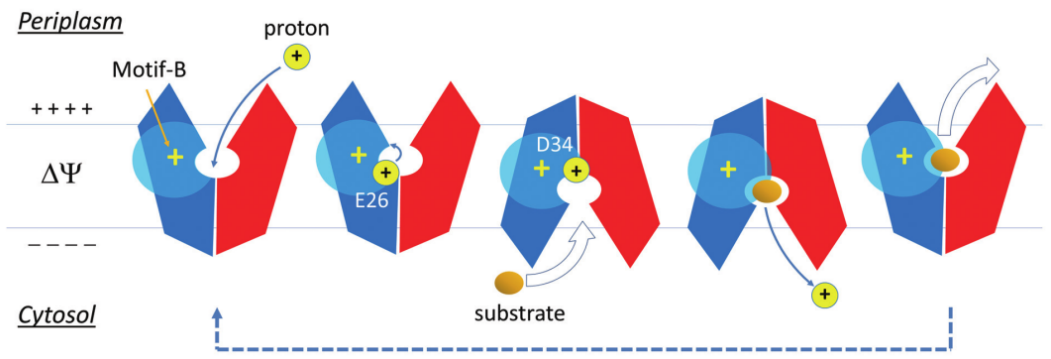

Figure 1.8 MdfA of E. coli (a) Structure of MdfA with chloramphenicol binds to it (PDB accession: 4ZOW). Chloramphenicol is shown as magenta stick model. Glu26 (nearer to periplasm) and Asp34 (nearer to cytoplasm) are marked as red spheres, and Arg112 as a blue sphere. The inward-facing cavity is shown as dot-surface representation. TMs are labelled with numbers in the right panel. (b) Proposed mechanism of proton translocation and drug transport in MdfA (adopted from Heng et al., 2015).

The high resolution structures of MdfA were only possible due to the serendipitous occurrence of the Q131R substitution, as the MdfA wild-type protein does not yield welldiffracting crystals. The presence of a cytoplasmic rim around the entrance to the multidrug recognition pocket has been suggested as an explanation for stabilization by the Q131R. Replacement to arginine partially disrupts the charge asymmetry and leads to electrostatic repulsion between the two halves of the rim, which possibly stabilize MdfA in a specific, partially inward-open conformation that aid high quality crystal formation (Zomot et al., 2018, Figure 1.10). 
More high resolution structures of MdfA with different conformations and ligands were obtained recently (Nagarathinam et al., 2018 ; Wu et al., 2019, Wu et al., 2020). The structure of MdfA in the outward open state was obtained by utilizing antibody Fab fragments as crystallization chaperones. Compared to the ligand-bound inward open state, the conformation of TM5 is kinked and twisted (Nagarathinam et al., 2018).

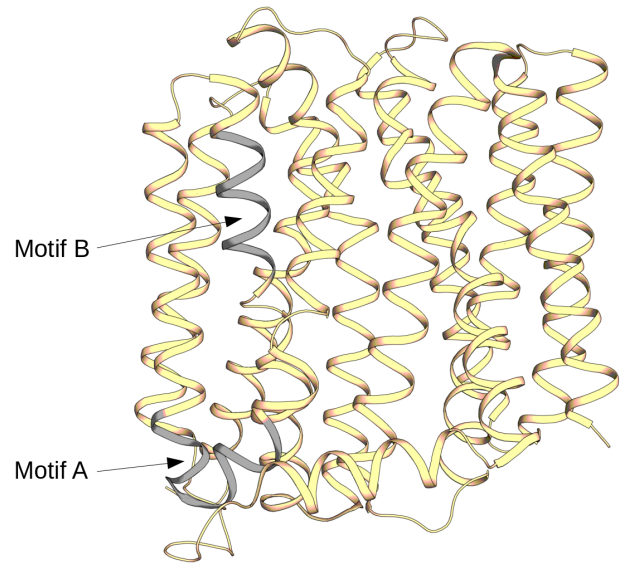

Figure 1.9 Location of motif $A$ and motif $B$ in MdfA (PDB accession: 6EUQ). Both motifs are highlighted in grey.

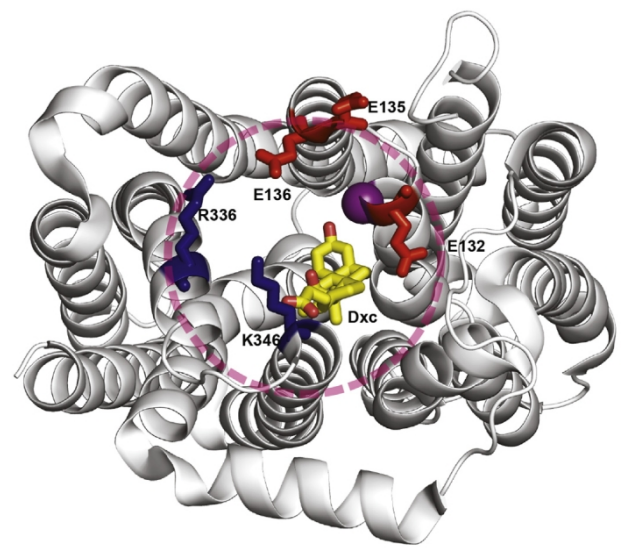

Figure 1.10 Cytoplasmic rim of MdfA. The imaginary rim is shown as a discontinuous pink circle. Basic and acidic residues are shown in blue and red sticks, respectively. The $\mathrm{C} \alpha$ atom of the arginine at position 131 is shown as a purple sphere. The substrate deoxycholate (Dxc) is in yellow sticks.

\subsubsection{Multidrug and Toxic Extrusion family}

Multidrug and toxic extrusion (MATE) transporters are divided into the DinF, NorM and eukaryotic subfamilies (Nair et al., 2016). Although they also possess a 12-TM organized into two bundles of six helices architecture, the topology of the TM segments is distinct from those of MFS (Figure 1.11), therefore the MATE and MFS proteins were classified as different protein families (Brown et al., 1999 ; Kusakizako et al., 2020). They use transmembrane $\mathrm{H}^{+}$and/or $\mathrm{Na}^{+}$gradients to drive the efflux of fluoroquinolones (norfloxacin), cationic dyes (acriflavine and ethidium) and aminoglycosides (kanamycin and streptomycin) and tigecycline (Kuroda and Tsuchiya, 2009). The crystal structures of $\mathrm{Na}^{+}$-dependent NorM transporters from Vibrio cholerae and Neisseria gonorrhoeae (He et al., 2010 ; Lu et al., 2013a), and $\mathrm{H}^{+}$-dependent DinF transporters from Pyrococcus furiosus and Bacillus halodurans (Tanaka et al., 2013 ; Lu et al., 2013b) have been reported. 

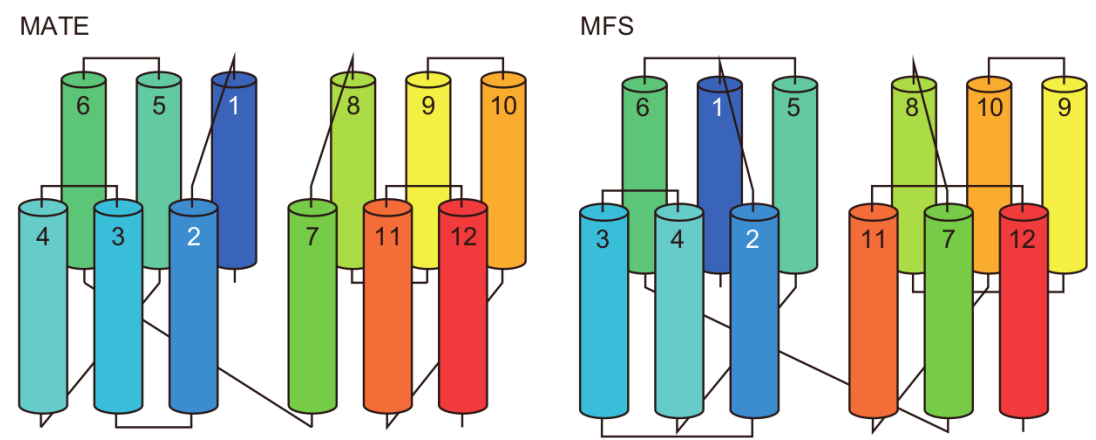

Figure 1.11 Topology diagram of MATE and MFS transporter. TM1-6 and TM7-12 form the N- and C-lobes, respectively (adopted from Kusakizako et al., 2020)

\subsubsection{Small Multidrug Resistance family}

The most well-studied member of the SMR family is EmrE from E. coli (Muth and Schuldiner, 2000). This small transporter with 110 amino acids consists of four TM helices. It catalyzes the transport of aromatic cations such as ethidium, methyl viologen, acriflavine and tetraphenylphosphonium in exchange with two protons (Rotem and Schuldiner, 2004). The first three TM helices form a substrate-binding chamber, whereas the fourth helix is mainly involved in dimerization interaction and confer stability to the dimer during the conformational changes of the pump between inward-facing and outward-facing in the transport cycle (Korkhov and Tate, 2009). NMR data shows that this drug/proton antiporter can simultaneously bind both substrates. A "free-exchange" kinetic model was proposed, which described EmrE as having multiple transport stoichiometries instead of a single $2: 1 \mathrm{H}^{+} / \mathrm{drug}$ antiport ratio (Robinson et al., 2017).

Through cryo-EM (Fleishman et al., 2006), crystallographic (Chen et al., 2007) and NMR data (Morrison et al., 2011), it is confirmed that EmrE is an antiparallel dimer, i.e. the monomers are inserted into two opposing directions in the membrane. It was shown that a single mutation in one of the two monomers disrupted the balance of inward- and outwardfacing proteins, which drastically reduced the function of EmrE in conferring drug resistance (Leninger et al., 2019). 
It was proposed that for dual-topology proteins such as EmrE, the entire protein subunit can flip completely in the membrane (Woodall et al., 2017). However, previous study also reported that the EmrE monomers attain stable topologies before dimerization, and the evolution has fine-tuned the polypeptide sequence that enables the monomers insert with equal probability $\left(\mathrm{N}_{\text {in }} / \mathrm{C}_{\text {in }}\right.$ or $\mathrm{N}_{\text {out }} / \mathrm{C}_{\text {out }}, \mathrm{N}$ - and $\mathrm{C}$-termini are either both inside or both outside the cell, respectively), suggesting that topological flip flop of EmrE in the membrane in vivo is not plausible (Fluman et al., 2017).

\subsection{Acinetobacter baumannii}

Acinetobacter baumannii is a Gram-negative aerobic coccobacillus which has become a major threat to public health in many countries. In 2017, the World Health Organization (WHO) has classified carbapenem-resistant $A$. baumannii as top of their priority list for pathogens that urgently need research and development of new antibiotics. Outbreaks related to $A$. baumannii occur frequently worldwide, including in Frankfurt where pan drugresistant A. baumannii was detected (Valencia et al., 2009 ; Göttig et al., 2014). Infections associated with $A$. baumannii such as ventilator-associated pneumonia, bloodstream infection, urinary tract infection, meningitis and wound infection are high in mortality rate. Patients that are immunocompromised and spend a prolonged period of time in hospital are at high risk to this opportunistic pathogen (Falagas and Rafailidis, 2007 ; Peleg, 2008).

Several characteristics of $A$. baumannii contribute to its persistence and host infection in clinical setting. A. baumannii could survive under harsh environment and able to form biofilm on biotic and abiotic surfaces, making it particularly difficult to eradicate in the hospital. Its genome plasticity provides major routes to gain resistance against most classes of antibiotics. Furthermore, the high level of intrinsic antibiotic resistance due to the presence of different classes of multidrug efflux pumps, together with the remarkably low outer membrane permeability make $A$. baumannii particularly difficult to eradicate (Dijkshoorn et al., 2007). 


\subsubsection{Genome plasticity of $A$. baumannii}

The presence of multiple mobile genetic elements such as insertion sequences (ISs), transposons, and resistance islands in $A$. baumannii contributes to its elevated genomic plasticity. This allows the accumulation of many resistance determinants and leads to the high incidence of multidrug resistance in this pathogen (Peleg, 2008 ; Pagano et al., 2016).

The largest resistance island known is AbaR1 in A. baumannii AYE responsible for outbreaks in France during 2004 (Fournier et al., 2006). The island contains 45 genes associated with antibiotic, antiseptic, and heavy metal resistance within an 86-kb region, including the genes encoding resistance to aminoglycosides (strA, strB, aphA1, aac69), putative tetracyclineresistance genes tet $A$ and its regulator tetR and the chloramphenicol-resistance gene $\mathrm{cm} / A$ (Fournier et al., 2006). AYE strain with deleted AbaR1 showed restored susceptibility to multiple classes of antibiotics, confirming the contribution of $\mathrm{AbaR}$ islands to multidrug resistance of AYE strain. Interestingly, the AbaR1 deletion stain with repaired comM gene also resulted in high level of natural transformability, which shows that the interruption of the comM gene by AbaR1 causes a decrease in natural transformation (Godeux et al., 2020).

The AbaR2 in ACICU strain is much shorter $(8.9 \mathrm{~kb})$ and encodes only seven resistance genes (lacono et al., 2008). A. baumannii strain MDR-ZJ06 widely spread in China harbours a 38.6-kb resistance island AbaR22. It contains 40 genes but only a few are antibiotic resistance genes (Zhou et al., 2011). In the clinical isolate AB5075 which is highly virulent, phage islands account for $7 \%$ of the chromosome. Many unique genes found in the phage islands with unknown function may include novel virulence genes (Gallagher et al., 2015).

Bacterial insertion sequences (IS) are the smallest (normally less than $2 \mathrm{~kb}$ ) and the most abundant transposable elements capable of independent transposition. Two copies of the same IS flanking a resistance gene form a structure called composite transposon that is able to move and contributes to antimicrobial resistance dissemination (Mahillon and Chandler, 1998). The composite transposon Tn2006 is one of the most common determinants of carbapenem resistance which is widely spread among clinical $A$. baumannii isolates and correlated with higher mortality (Lee et al., 2012 ; Pagano et al., 2016). 
Multiple studies showed that IS elements enhance $\beta$-lactamase gene expression by providing promoters. ISAba1 has frequently been found upstream of the AmpC $\beta$-lactamase and OXA carbapenemase genes in A. baumannii (Poirel and Nordman, 2006 ; Mugnier et al., 2009 ; El-Shazly et al., 2015). IS element transposition into a targeted gene can inactivate or increase certain gene products. For example insertion mutation within the carO gene by the ISAba1, ISAba125, or ISAba825 element reduced the production of this porin and causes carbapenem resistant (Mussi et al., 2005 ; Lee et al., 2011). The disruption of the efflux pump regulatory genes by IS causes the overexpression of efflux pump genes, as in the case of mutation on AdeRS by ISAba1 that contributes to overexpression of ade $B$ and subsequently tigecycline resistance (Sun et al., 2012).

\subsubsection{Multidrug efflux pumps of $A$. baumannii}

\subsubsection{RND systems in A. baumannii}

AdeABC is the first pump described in $A$. baumannii (Magnet et al., 2001). It confers resistance to aminoglycosides, tetracyclines, fluoroquinolones, chloramphenicol, trimethoprim and tigecycline (Magnet et al., 2001 ; Peleg et al., 2007 ; Ruzin et al., 2007). The overproduction of AdeABC is mainly responsible for multidrug resistance by antibiotic efflux in clinical isolates (Yoon et al., 2013). An adeC-inactivated mutant displays resistance to substrates in a similar pattern with the parental strain (Marchand et al., 2004), suggesting that AdeC is not essential for the efflux function of AdeABC. AdeC is absent in $41 \%(48 / 116)$ of clinical isolates carrying adeRS-adeAB operon (Nemec et al., 2007), indicating that AdeAB could utilize another outer membrane protein to form a functional tripartite complex, as in the case of Pseudomonas aeruginosa where the outer membrane protein OprM is produced independently and interplays with several RND pumps (Zhao et al., 1998). It was suggested that AdeAB of $A$. baumannii ATCC 17978 engages AdeK for its activity (Leus et al., 2018).

Expression of ade $A B C$ is tightly regulated by the two-component regulatory system AdeRAdeS located upstream of adeABC and transcribed in the opposite direction (Marchand et al., 2004, Figure 1.12). The histidine kinase AdeS senses environmental stimuli, while the response regulator AdeR mediates the cellular response by receiving a phosphoryl signal 
from AdeS and further stimulates the expression of its target genes (West and Stock, 2001 ; Groisman et al., 2016). Single amino acid mutations in adeR and adeS are identified as the major cause of adeABC overexpression (Merchand et al., 2004 ; Yoon et al., 2013 ; Sun et al., 2016 ; Gerson et al., 2018). Another mechanism that leads to the overexpression of ade $A B C$ involves the transposition of insertion sequence (IS), ISAba1 into adeS, which stimulates AdeR to activate the adeABC promoter (Ruzin et al., 2007 ; Sun et al., 2012). The tight correlation of the AdeRS system with expression of adeABC makes it a potential therapeutic target against multidrug resistant $A$. baumannii. The crystal structure of AdeR in complex with DNA provides detailed mechanistic insight that could aid the drug design targeting AdeR (Wen et al., 2017).

A second RND system, AdelJK, was characterized in the clinical isolate A. baumannii BM4454. It contributes to intrinsic resistance to various drugs, including $\beta$-lactams (ticarcillin, cephalosporins and aztreonam), fluoroquinolones (moxifloxacin, pefloxacin and levofloxacin) tetracyclines, tigecycline, lincosamides, rifampin, chloramphenicol, novobiocin, and fusidic acid (Damier-Piolle et al., 2008). Besides antibiotics, it can also export acridine, safranin, pyronine, and sodium dodecyl sulfate. Inactivation of AdelJK in A. baumannii ATCC 17978 did not cause susceptibility to aminoglycosides such as kanamycin and gentamicin, azithromycin, vancomycin, and trimethoprim, while strongly affects the activities of carbenicillin and cloxacillin (Leus et al., 2018).

The toxicity observed with $E$. coli and $A$. baumannii when the adelJK operon was overexpressed suggests that the expression of adelJK is tightly regulated (Damier-Piolle et al., 2008). No open reading frames (ORFs) coding for regulatory proteins were found in the vicinity of the adelJK operon (Figure 1.12). Through whole genome sequencing, it was identified that AdeN, a transcription regulator belonging to the TetR family represses the expression of the adelJK operon (Rosenfeld et al., 2012). Inactivation of adeN by ISAba1 and adeN knockout in A. baumannii ATCC 17978 eliminate the transcriptional repression of adelJK, which increases the virulence of the strains and lead to higher larval mortality rate (Saranathan et al., 2017). The insertion of ISAba125 and ISAba27 into adeN were also observed in A. baumannii isolates with enhanced drug resistance (Gerson et al., 2018). Besides IS elements, several different mutations in adeN, for example deletions and premature stop codons, led to increased tigecycline MICs (Gerson et al., 2018). 
AdeFGH system is the third RND efflux system discovered through the comparison of the deletion mutant ( $\triangle a d e A B C \triangle a d e I J K)$ which exhibited resistance on chloramphenicol with the parental strain (Coyne et al., 2010a). The operon of adeFGH is widespread amongst clinical isolates of $A$. baumannii. An ORF for a putative LysR-type transcriptional regulator (LTTR), named AdeL, is located upstream of the adeFGH operon and transcribed in the opposite direction (Figure 1.12). Adel point mutations lead to a significant increase in adeG expression (Coyne et al., 2010b). The substrates of AdeFGH include chloramphenicol, clindamycin, fluoroquinolones, trimethoprim, tetracyclines, tigecycline, and sulfonamide (Coyne et al., 2010b; Cortez-Cordova and Kumar, 2011). Besides antibiotics, AdeFGH is able to export SDS, acridine orange, safranine $\mathrm{O}$, and ethidium bromide (Coyne et al., 2010b). However, it was reported that deletion of adeFGH does not affect the antimicrobial susceptibility profile of $A$. baumannii, suggesting that this RND system does not play important role in antimicrobial resistance (Amin et al., 2013).

The biofilm formation of $A$. baumannii clinical isolates was associated with the overexpression of adeFGH and autoinducer synthase abal in the presence of levofloxacin and meropenem at sub-MICs (He et al., 2015). Another study also correlated biofilm formation with AdeFGH and Abal in the presence of colistin (Sato et al., 2018). The most common mutations observed in the biofilm populations of $A$. baumannii ATCC 17978 exposed to ciprofloxacin are in the repressor gene adeL, which regulates AdeFGH (SantosLopez et al., 2019). It was postulated that the overexpression of AdeFGH induced by the presence of antibiotic accelerates the synthesis and transport of quorum sensing molecules acyl homoserine lactone (AHL) during biofilm formation in A. baumannii (He et al., 2015).

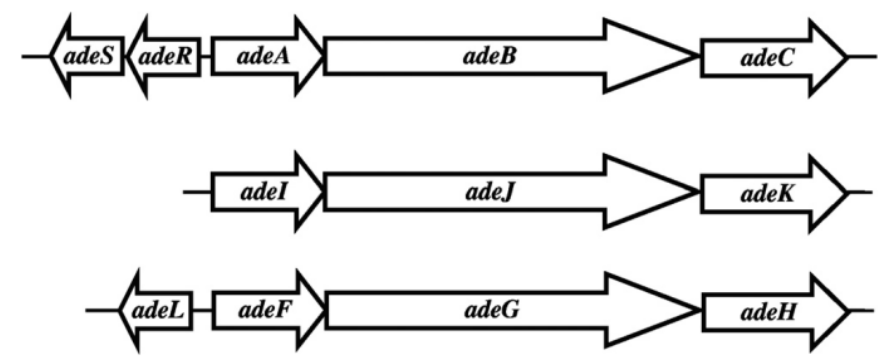

Figure 1.12 Operons encoding RND efflux systems in A. baumannii. AdeR-AdeS controls the expression of ade $A B C$, no ORF coding for regulatory proteins were found near to adelJK operon, AdeL regulates the expression of adeFGH. Open arrows indicate direction of transcription (adopted from Coyne et al., 2011). 
The AdeDE pump has been reported in Acinetobacter genomic DNA group 3 (GDG3) conferring resistance to amikacin, ceftazidime, chloramphenicol, ciprofloxacin, erythromycin, ethidium bromide, meropenem, rifampicin and tetracycline (Chau et al., 2004). The sequence downstream to adeDE was not an ORF encoding the outer membrane protein, suggested that AdeDE may form a tripartite complex with other outer membrane proteins (Chau et al., 2004). This RND-type efflux pump was first detected in A. baumannii GDG3 (Chau et al., 2004 ; Chu et al., 2006) and AdeRS-AdeABC/AdelJK negative isolates (Lin et al., 2009b). It was assumed that adeDE does not co-exist with ade $A B C$ in $A$. baumannii, but currently it was reported that ade $E$ is found to co-exist with ade $B$ in some strains (Hou et al., 2012). The exact function and regulatory aspects of adeDE in $A$. baumannii remains unclear.

\subsubsection{Other non-RND efflux pumps of $A$. baumannii}

The MFS-type specific transporter, tetracycline (Tet) efflux pump is widely presence in many clinical isolates of A. baumannii (Ribera et al., 2003a ; Vila, 2007 ; Akers et al., 2009). The tet(B) gene which confers resistance to both tetracycline and minocycline is more prevalent than $\operatorname{tet}(A)$ in tetracycline-resistant $A$. baumannii strains (Huys et al., 2005 ; Wang et al., 2017). Both pumps are reported to be ineffective against glycylcyclines such as tigecycline (Vila et al., 2007). Another pump Tet39 was characterized in a clinical isolate and environmental isolates of Acinetobacter strains in Denmark and Thailand, conferring resistance to tetracycline but not to minocycline (Agerso and Guardabassi 2005 ; Agerso and Peterson 2007). However, Tet39 found on a tetracycline-resistant clinical strain A. baumannii RCH52 exhibits reduced susceptibility also on minocycline (Hamidian et al., 2016). Another MFS-type specific transporter is the chloramphenicol transporter such as the CmIA (Fournier et al., 2006) and CraA (Roca et al., 2009). CraA with an amino acid similarity of about $61 \%$ to the MdfA of $E$. coli is widely present in $A$. baumannii isolates. Deletion of CraA in $A$. baumannii ATCC 19606 resulted in a 128-fold decrease in MIC value of chloramphenicol (Roca et al., 2009).

AbeM belongs to the MATE family was shown to confer resistance to fluoroquinolones, gentamicin, doxorubicin and triclosan (Su et al., 2005). The hydrophilic fluoroquinolones (norfloxacin and ciprofloxacin) appeared to be better substrates for the AbeM pump than the hydrophobic fluoroquinolone (ofloxacin). AbeM also facilitates the efflux of dyes such as 
acriflavin, Hoechst 33342, rhodamine 6G, and ethidium bromide. AbeM was confirmed to be a $\mathrm{H}^{+}$-driven pump, instead of $\mathrm{Na}^{+}$-driven pump ( $\mathrm{Su}$ et al., 2005). It was speculated that AdeABC, AdelJK and AbeM contribute to imipenem resistance cooperatively in the clinical isolates of $A$. baumannii, based on the observation that imipenem-resistant strain shows 4-, 3- and 11-fold increases in adeB, adeJ and abeM expression, respectively, compared to the imipenem-sensitive strain (Hou et al., 2012).

AbeS belongs to the SMR family efflux and is a drug/ $\mathrm{H}^{+}$antiporter with 109 residues that displays homology with the EmrE of $E$. coli $(71 \%$ similarity). Deletion of abeS in $A$. baumannii and expression of abeS in E. coli KAM32( $\triangle a c r A B \Delta m d t K)$ indicated that it confers low level resistance to chloramphenicol, fluoroquinolones, erythromycin, and novobiocin and resistance to dyes and detergents (Srinivasan et al., 2009). Increased expression of abeS is reported to be related with amikacin resistance (Lin et al., 2015). AbeS was also shown to confer resistance toward ethidium, acriflavine and benzalkonium in an E. coli $\triangle e m r E \Delta m d f A$ background (Lytvynenko et al., 2016).

The Acinetobacter chlorhexidine efflux protein (Acel) is the first characterized prototype of the PACE family of multidrug efflux pumps (Hassan et al., 2013, Hassan et al., 2015). Efflux pumps of PACE family consist of about 150 amino acid residues with four TM helices, they possess a limited substrate range, which include primarily the synthetic biocide chlorhexidine and acriflavine (Pérez-Varela et al., 2019). The acel gene is under the control of AceR, a LysR-type transcriptional regulator (Liu et al., 2018).

Recently it was demonstrated that short-chain diamines that play vital roles in bacterial physiology and virulence are substrates for the PACE family (Hassan et al., 2019). It is also reported that Acel exist in monomers and dimers at low $\mathrm{pH}$ (5 and 6), and primarily in dimers when the $\mathrm{pH}$ was increased from 7 to 9 . A significant increase in the dimer population was also observed when chlorhexidine was added to the protein. The binding to chlorhexidine facilitates the functional form of the protein (Bolla et al., 2020). Since this novel family of transporters are highly conserved in other Gram-negative pathogens, their physiological roles other than drug resistance remain to be determined (Hassan et al., 2018). 


\subsubsection{Outer membrane proteins of $A$. baumannii}

In Gram-negative bacteria, the uptake of antibiotics is slowed by the barrier of outer membrane, consists of asymmetric bilayer of lipopolysaccharides and phospholipids. The embedded outer membrane protein channels, termed porins, regulate the drug permeability. Most small and hydrophilic antibiotics such as the $\beta$-Lactams, tetracyclines, chloramphenicol, and fluoroquinolones reach the periplasm through porins. The relatively large and lipophilic drugs such as macrolides, rifamycins, novobiocin, or fusidic acid diffuse across the asymmetric lipid bilayer (Nikaido and Pagès, 2012 ; Zgurskaya et al., 2015).

Similar to $P$. aeruginosa, $A$. baumannii produce porins which provide very low permeability for solutes across the outer membrane. A. baumannii therefore lacks high-permeability, nonselective trimeric porins such as $\mathrm{OmpF}$ and $\mathrm{OmpC}$ that are typically found in E. coli and other Enterobacteriaceae. It is estimated that the permeability of $P$. aeruginosa and $A$. baumannii outer membranes is only 1 to $8 \%$ of that E. coli (Nikaido, 2003; Sugawara and Nikaido 2012 ; Zgurskaya et al., 2015). Low outer membrane permeability lowers the permeability rate of toxins into the cell and prevents high intracellular antibiotic concentrations in conjunction with RND-type multidrug efflux pumps. This enables acquisition of more potent resistance mechanisms such as upregulation of further multidrug efflux pumps or by enzymatic degradation of antibiotics (Masi et al., 2017).

The most abundant porin in the outer membrane of $A$. baumannii is outer membrane protein A (OmpA), a $40 \mathrm{kDa}$ protein belonging to the OmpA-like family (Jyothisri et al., 1999). It is highly homologous to OmpA of Enterobacteriaceae and OprF of Pseudomonas spp (Sugawara et al., 1996). OmpA is the principal slow-diffusion porin of $A$. baumannii. Strains lacking ompA have 2-3 fold decreased outer membrane permeability for cephalothin and cephaloridine (Sugawara and Nikaido, 2012) but are on the other hand more sensitive to aztreonam, nalidixic acid, chloramphenicol, trimethoprim and other drugs (Smani et al., 2014 ; Kwon et al., 2017). For the latter phenotype, it has been suggested that OmpA might be an outer membrane component of a yet to be defined (tripartite) efflux pump (Smani et al., 2014). OmpA was, however, also reported to selectively facilitate the entry of small molecules such as sulbactam, imipenem, and ETX2514 ( $\beta$-lactamase inhibitor). Small modifications in the structure of ETX2514 could improve its passage through OmpA, 
suggesting that drug design to optimize the entry through OmpA could be a promising solution to solve multidrug resistance in A. baumannii (lyer et al., 2018).

OmpA is highly conserved among different strains of $A$. baumannii (lyer et al., 2018). In general, ompA plays an important role in maintaining the outer membrane structure. It is also involved in biofilm formation on plastic surface (Gaddy et al., 2009). It has multiple functions in bacterial pathogenesis, including adherence and invasion of epithelial cells and induction of apoptosis in host cell (Choi et al., 2007 ; Choi et al., 2008 ; Lee et al., 2010). A. baumannii is able to secrete OmpA via outer membrane vesicles to host cell, inducing apoptosis and cell death (Jin et al., 2011). As an important virulence factor, OmpA has become a target for vaccines and antibodies development (Luo et al., 2012 ; Nie et al., 2020).

Several other porins have been reported to be associated with carbapenem resistance in $A$. baumannii, including CarO (carbapenem resistance-associated outer membrane protein), Omp33-36 and OprD homolog. Disruptions of the carO gene by insertion elements were observed in imipenem-resistant strains (Lee et al., 2011). Early reports suggested that this $29 \mathrm{kDa}$ pore-forming protein has an unspecific monomeric channel function rather than a specific function (Mussi et al., 2005 ; Siroy et al., 2005). CarO was shown to allow the selective uptake of the ornithine, other basic amino acids and carbapenems (Mussi et al., 2007). It was further identified that CarO channels possess an imipenem, but not meropenem binding site (Catel-Ferreira et al., 2011). However, another study claimed that CarO is able to transport small amino acids but not large compounds such as imipenem and meropenem (Zahn et al., 2015).

Omp 33-36 is a 33 to $36 \mathrm{kDa}$ outer membrane protein that is also frequently related to carbapenem resistance in A. baumannii (Clark 1996 ; Costa et al., 2000 ; del Mar Tomás et al., 2005). It allows the passage of water and plays an important role for fitness and virulence in $A$. baumannii. The knockout strain lacking this porin shows defective growth rate and significantly reduced capability of adherence, invasion, and cytotoxicity. Omp33-36 induces apoptosis through caspase activation and subsequently modulates autophagy (Smani et al., 2013, Rumbo et al., 2014). A study warned that treatment with subinhibitory concentrations of carbapenems decreased the mRNA levels of omp33-36, indicating that 
inadequate dosing of carbapenem therapy could lead to the development of reversible resistance (Novović et al., 2018).

OprD of Pseudomonas aeruginosa is a well-characterized channel for imipenem influx and reduced or loss of OprD causes low permeation of carbapenems (Trias and Nikaido 1990; Li et al., 2012). OprD homologue in A. baumannii was reported to have the same influence in carbapenem resistance (Dupont et al., 2005 ; Fernández-Cuenca et al., 2011). OprD and CarO were found to be downregulated in a clinical isolate of $A$. baumannii resistance to imipenem (Luo et al., 2011). However, other studies showed that the lack of OprD in $A$. baumannii did not affect imipenem and meropenem susceptibilities but more likely to affect the adaptation of $A$. baumannii to magnesium- and/or iron-depleted environments (CatelFerreira et al., 2012; Smani and Pachón, 2013). The discrepancy could be due to the presence of four OprD orthologs, renamed OccAB1 to OccAB4 (Zahn et al. 2016). According to their structural and functional assays, OprD (OccAB1) could translocate all investigated antibiotics (including imipenem and meropenem) and the OccAB family is the major contributor to the uptake of small molecules in A. baumannii (Zahn et al., 2016).

\subsection{Resistance to the last-resort antibiotic tigecycline in A. baumannii}

Currently the last-resort antibiotics for severe infections related to carbapenem-resistant $A$. baumannii are colistin and tigecycline. Tigecycline which belongs to the third generation tetracyclines was approved for clinical use in 2006 for the treatment of complicated skin and soft tissue and abdominal infections in humans (Giamarellou and Poulakou, 2011). Besides multidrug-resistant $A$. baumannii, it is also used as a last-resort antibiotic for combating extended-spectrum $\beta$-lactamase-producing Enterobacteriaceae (ESBL), carbapenemresistant Enterobacteriaceae (CRE), methicillin-resistant Staphylococcus aureus (MRSA), and vancomycin-resistant Enterococcus (VRE) (Petersen et al., 1999 ; Pankey, 2005). 


\subsubsection{Tetracyclines resistance mechanisms}

Discovered in the 1940s, the first generation, tetracyclines, i.e. chlortetracycline and oxytetracycline, are produced naturally by Streptomyces aureofaciens and Streptomyces rimosus, respectively. The second generation consists of semisynthetic tetracyclines, such as minocycline and doxycycline, with improved antimicrobial potency (Figure 1.13) (Nelson and Levy, 2011 ; Nguyen et al., 2014). The third generation tetracyclines include tigecycline, omadacycline and eravacycline (Figure 1.13). Tetracyclines exhibit bacteriostatic activity against Gram-positive and Gram-negative bacteria through reversible binding to the A site of the $30 \mathrm{~S}$ subunit, thereby preventing the binding of aminoacyl-tRNA to the ribosomal A site and consequently inhibiting protein synthesis (Chopra and Roberts 2001).

Due to their broad spectrum of activity and relatively low cost, tetracyclines are used extensively in human and animal infections. In many countries, tetracyclines are incorporated into livestock feed at subtherapeutic doses as growth promoters for metaphylaxis purposes (Granados-Chinchilla and Rodríguez, 2017). The misuse of tetracyclines has led to fast increase in acquired tetracycline resistance. Tetracycline resistance genes in bacteria are typically located in mobile plasmids, transposons, conjugative transposons, and integrons, enabling the genes to move between species and into a wide range of genera by conjugation (Chopra and Roberts 2001).

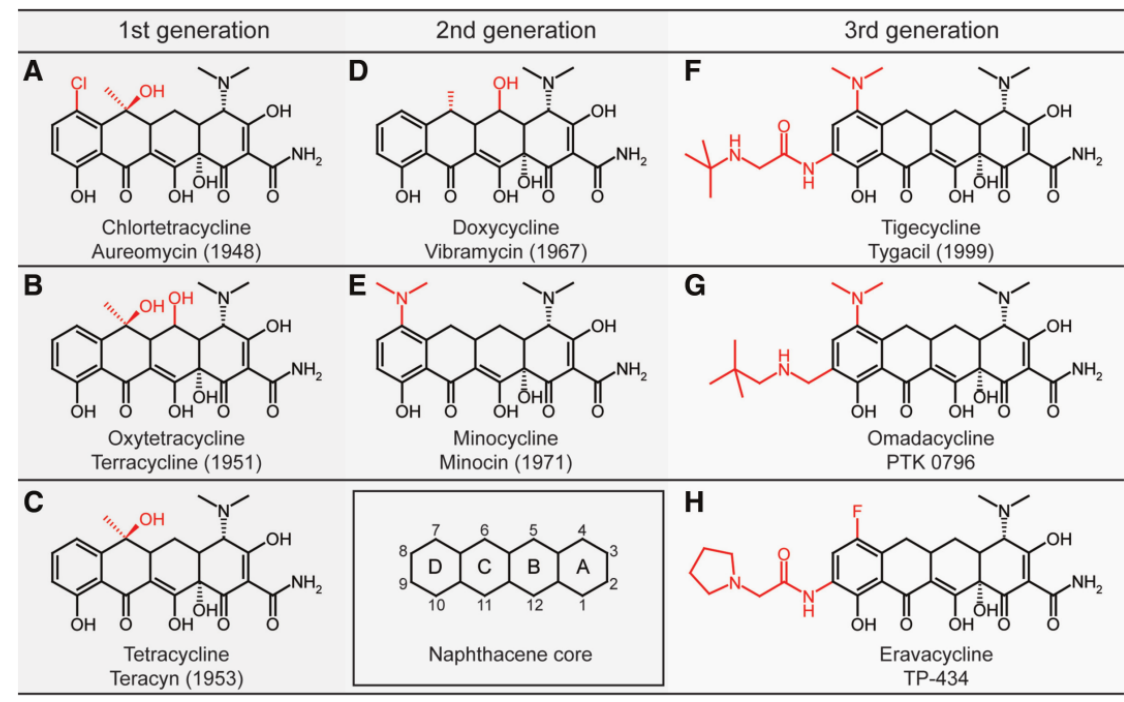

Figure 1.13 Chemical structures of first, second and third generation of tetracyclines (adopted from Nguyen et al., 2014) 
The majority of the tetracycline resistance phenotype is caused by genes encoding approximately $46 \mathrm{kDa}$ MFS-type Tet efflux pumps (Table 1.1). The Tet protein is the first efflux pump identified to confer antibiotic resistance (Levy, 1978 ; McMurry et al., 1980). In Gram-negative bacteria, this MFS-type drug/ $/ \mathrm{H}^{+}$antiporter consists of $12 \mathrm{TM}$ helices with long central nonconserved cytoplasmic loops connecting transmembrane TM6 and TM7 (Yamaguchi et al., 1990). In Gram-positive bacteria, tetracycline efflux pumps with 14 TM helices also exist, for example TetK from Staphylococcus aureus (Guay et al., 1993). The expression of the tetracycline efflux pump gene is controlled through a repressor, TetR. Without tetracycline, TetR binds to the target operator and represses the transcription of efflux pump. It is released in the presence of tetracycline to allow expression of efflux pump (Hillen and Berens 1994).

Table 1.1 Tet resistance determinants. The latest update is in October 2020, adopted from http://faculty.washington.edu/marilynr/tetweb4.pdf

\begin{tabular}{|c|c|c|c|c|c|}
\hline Efflux & & & $\begin{array}{l}\text { Ribosomal } \\
\text { protection }\end{array}$ & $\begin{array}{l}\text { Enzymatic } \\
\text { degradation }\end{array}$ & Unknown \\
\hline $\operatorname{tet}(A)$ & tet(30) & $\operatorname{tet}(57)$ & $\operatorname{tet}(M)$ & $\operatorname{tet}(X 1)-\operatorname{tet}(X 7)$ & $\operatorname{tet}(U)$ \\
\hline $\operatorname{tet}(B)$ & tet(31) & $\operatorname{tet}(58)$ & $\operatorname{tet}(O)$ & $\operatorname{tet}(34)$ & \\
\hline $\operatorname{tet}(C)$ & tet(33) & $\operatorname{tet}(59)$ & $\operatorname{tet} B(P)$ & $\operatorname{tet}(37)$ & \\
\hline $\operatorname{tet}(D)$ & tet(35) & $\operatorname{tet} A(60)$ & $\operatorname{tet}(\mathrm{Q})$ & $\operatorname{tet}(47)$ & \\
\hline $\operatorname{tet}(E)$ & tet(38) & tetB(60) & tet $(S)$ & $\operatorname{tet}(48)$ & \\
\hline $\operatorname{tet}(G)$ & tet(39) & tet(62) & $\operatorname{tet}(T)$ & tet(49) & \\
\hline $\operatorname{tet}(H)$ & $\operatorname{tet}(40)$ & tet(63) & $\operatorname{tet}(W)$ & $\operatorname{tet}(50)$ & \\
\hline $\operatorname{tet}(J)$ & tet(41) & tet(64) & tet(32) & $\operatorname{tet}(51)$ & \\
\hline $\operatorname{tet}(K)$ & tet(42) & $\operatorname{otr}(B)$ & $\operatorname{tet}(36)$ & $\operatorname{tet}(52)$ & \\
\hline $\operatorname{tet}(L)$ & tet(43) & $\operatorname{otr}(C)$ & tet(44) & tet(53) & \\
\hline $\operatorname{tet} A(P)$ & $\operatorname{tet}(45)$ & tcr3 & $\operatorname{tet}(61)$ & $\operatorname{tet}(54)$ & \\
\hline $\operatorname{tet}(V)$ & $\operatorname{tet} A(46)$ & & $\operatorname{otr}(A)$ & $\operatorname{tet}(55)$ & \\
\hline $\operatorname{tet}(Y)$ & tetB(46) & & & tet(56) & \\
\hline $\operatorname{tet}(Z)$ & & & & $\operatorname{tet}(57)$ & \\
\hline
\end{tabular}

Some tetracycline resistance determinant genes encode for ribosomal protection proteins (RPPs) which are approximately $72.5 \mathrm{kDa}$. The best known examples are TetM and TetO, which were first identified in Streptococcus spp. and Campylobacter jejuni, respectively (Connell et al., 2003). RPPs have homology to elongation factors EF-G, both compete for binding on the ribosomes. The binding of RPPs to the ribosome causes an alteration in ribosomal conformation which causes dissociation of tetracycline from the ribosome and 
prevents rebinding, without altering or stopping protein synthesis (Dönhöfer et al., 2012 ; Li et al., 2013).

Another less-frequent resistance mechanism is the enzymatic alteration of tetracyclines by genes such as tet $(X)$ and tet(37). These genes encode flavin-dependent monooxygenases. These enzymes utilize NADPH and oxygen to hydroxylate position C11a located between ring $B$ and $C$ of tetracyclines, resulting in an unstable compound with low affinity for the ribosomal target site and it furthermore is prone to non-enzymatic decomposition (Nguyen et al., 2014, Figure 1.14). Despite the fact that it is an oxygen-dependent reaction, the tet $X$ gene was first identified in transposons Tn4351 and Tn4400 harboured by the obligate anaerobe Bacteroides fragilis (Speer et al., 1991 ; Yang et al., 2004).
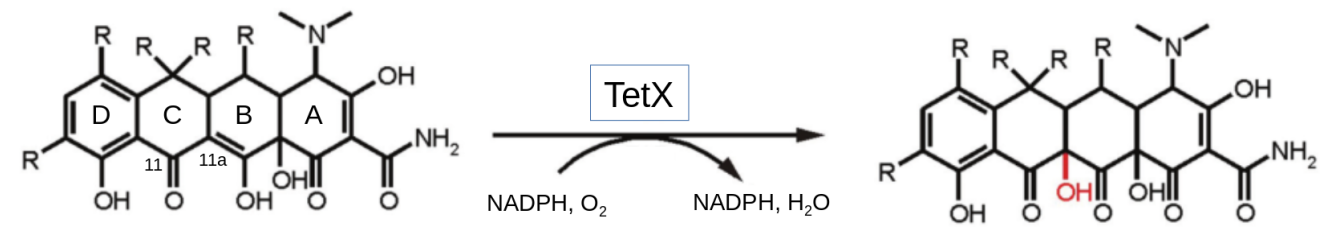

Figure 1.14 Enzymatic reaction catalyzed by TetX which requires NADPH and oxygen (modified from Nguyen et al., 2014).

\subsubsection{Tigecycline resistance}

In order to overcome tetracycline resistance to the first and second generations of tetracyclines via efflux, new drugs were developed by modification of the existing tetracyclines. Tigecycline is the first drug in the glycylcycline class of antibiotics (Figure 1.10) (Sum et al., 1993 ; Petersen et al, 1999). Although structurally related to minocycline, the addition of 9-t-butylglycylamido moiety at position $\mathrm{C} 9$ of the four-ring structure has enabled tigecycline to overcome the tetracycline resistance mechanisms. The affinity of tigecycline for the ribosomal target is in addition five times higher when compared to the affinities of minocycline or tetracycline (Bergeron et al., 1996 ; Olson et al., 2006). Stacking interactions between the 9-t-butylglycylamido group of tigecycline and the 16S rRNA of the 30S ribosome subunit enhance the binding affinity compared to other tetracycline antibiotics (Jenner et al., 2013). Binding affinity alone is not sufficient to overcome a ribosomal protection mechanism by proteins such as TetM. Structural studies however demonstrated that the 9-tbutylglycylamido group of tigecycline prevents the correct interaction between 16S rRNA and 
the loop III of domain IV of the TetM protein, once the drug is bound to the ribosome (Dönhöfer et al., 2012 ; Jenner et al., 2013). Apart from that, tigecycline is not a substrate for TetA pumps, most likely due the steric hindrance caused by the large substituent at position 9 (Someya et al., 1995 ; Hirata et al., 2004). However, tigecycline is a substrate for the RND pumps such as AcrAB or AcrEF (Hirata et al., 2004).

Unfortunately, tigecycline resistance has been reported frequently since its first use in the clinics (Liu et al., 2016). In Gram-negative bacteria, resistance to tigecycline is mainly caused by the overexpression of efflux pumps of the RND superfamily. Several studies demonstrated that overexpression of the RND-type pumps, especially the adeABC resulted in reduced tigecycline susceptibility in A. baumannii (Peleg et al., 2007 ; Hornsey et al., 2010). Although tigecycline is generally not a substrate of tetracycline efflux pumps, double frame-shift mutation in encoding regions of the interdomain loop region of the TetA efflux pump associated with transposon $\operatorname{Tn} 1721$ have been shown to reduce susceptibility to tigecycline in Salmonella spp (Tuckman et al., 2000 ; Akiyama et al., 2013).

Tigecycline resistance through ribosomal protection is still not clinically relevant in $A$. baumannii, although the tet(M) gene was found in some clinical isolates (Ribera et al., 2003b). Deletion of leucine-505 from loop III of domain IV in TetM led to the increase of tigecycline MIC. However, the mutation causes sensitivity to other tetracyclines (Linkevicius et al., 2016).

Another resistance mechanism of tigecycline is through the enzymatic inactivation of tigecycline by TetX. Hydroxylation of tigecycline by TetX to 11a-hydroxytigecycline has decreased the potency of this drug. Recently, several studies reported the emergence of tetX-mediated tigecycline resistance (He et al., 2019 ; Sun et al., 2019 ; Wang et al., 2020 ; He et al., 2020 ; Zhang et al., 2020). Plasmid-mediated tet(X3), tet(X4) and tet(X5), were found in numerous Enterobacteriaceae and $A$. baumannii isolated from the environment, animal farms and hospitals in China. The gene products are able to inactivate all tetracyclines, including tigecycline, eravacycline and omadacycline. The tigecycline MIC values for $A$. baumannii increased by 64 - to 128 -fold through this resistance mechanism $(\mathrm{He}$ et al., 2020). 


\subsection{Aims of the work}

The presence of various chromosomally encoded or acquired efflux pumps is one of the main factors contributing to multidrug resistance in $A$. baumannii. The RND tripartite systems (AdeABC, AdeEFG and AdelJK) which span the inner and outer membrane expel broad spectrum of toxic compounds out of the cells with high efficiency. Single-component efflux pumps of MFS, MATE, SMR and PACE family also plays important role in conferring specific or multidrug resistance.

One of the aims of this work is to investigate whether the deletion of a specific efflux pump would affect the expression of other efflux pumps which are important in conferring antibiotic resistance. The differential gene expression of RND efflux pumps (adeB, adeG, and adeJ), $\operatorname{cra} A$, and the outer membrane protein ompA in the background of RND knockout strains ( $\triangle$ ade $A B$ and $\triangle a d e l J)$ and an MFS-type transporter knockout strain $(\triangle c r a A)$ was analyzed. In addition, the differential gene expression when the knockout strains were exposed to subinhibitory concentration of chloramphenicol were determined.

Second aim of this work is to characterize five MFS-type putative chloramphenicol transporters of $A$. baumannii strain AYE. Among the five MFS-type transporters, CraA which is widely present in clinical isolates of $A$. baumannii, is particularly of our interest. It was identified as the homolog of the well-studied multidrug efflux transporter MdfA from $E$. coli, but reported to be a specific chloramphenicol transporter from $A$. baumannii (Roca et al., 2009). The main focus is to characterize the substrate specificity of CraA and to get insights into the protonation and drug transport mechanism.

Third aim of this work is to characterize three tetracycline transporters (TetA, TetG and $\operatorname{Tet} A(A)$ ) of $A$. baumannii strain AYE. The activity of these transporters against tetracycline, minocycline, doxycycline and the last-resort antibiotic tigecycline was investigated. In addition, the cooperativity between TetA and the RND tripartite systems in extruding tigecycline was also investigated through differential gene expression analysis of the RND efflux pumps (ade $B$, ade $G$ and adeJ) and tet $A$ when $A$. baumannii strain AYE was exposed to tigecycline. 


\section{CHAPTER 2 : MATERIALS AND METHODS}

\subsection{Materials}

\subsubsection{Lab equipments}

\begin{tabular}{|l|l|}
\hline Incubator & $\begin{array}{l}\text { Plate incubator (Heraeus Instrument) } \\
\text { HT Multitron (Infors) } \\
\text { C24 Incubator Shaker (New Brunswick Scientific) } \\
\text { Thermo MaxQ 4000 (Thermo Scientific) }\end{array}$ \\
\hline Centrifugation & $\begin{array}{l}\text { Heraeus Multifuge 1S-R (Thermo Scientific) } \\
\text { Heraeus Biofuge 13 (Thermo Scientific) } \\
\text { Sorvall Evolution RC (Thermo Scientific) } \\
\text { Sorvall WX UltraSeries (Thermo Scientific) } \\
\text { Optima XPN-100 (Beckman Coulter) } \\
\text { Optima TLX-120 (Beckman Coulter) }\end{array}$ \\
\hline Rotor & $\begin{array}{l}\text { FiberLite F8-6x1000y (Thermo Scientific) } \\
\text { FiberLite F14-6x250y (Thermo Scientific) } \\
\text { Thermo Scientific T647.5 (Thermo Scientific) }\end{array}$ \\
\hline Thermo Scientific T865 (Thermo Scientific) \\
Beckman Ti45 (Beckman Coulter) \\
Beckman Ti60 (Beckman Coulter) \\
Beckman TLA100 (Beckman Coulter)
\end{tabular}




\subsubsection{Chemicals and antibiotics}

Chemicals for media and buffers preparation were obtained from AppliChem, NeoLab, Sigma-Aldrich, Carl Roth, Merck or Life Technologies unless specifically mentioned otherwise.

\begin{tabular}{|l|l|}
\hline Antibiotic / Disinfectant / Dye & Source \\
\hline ACMA (9-amino-6-chloro-2-methoxyacridine) & Thermo Fisher Scientific \\
\hline Ampicillin & Carl Roth \\
\hline Benzalkonium chloride & Sigma-Aldrich \\
\hline Carbenicillin & AppliChem \\
\hline Chloramphenicol & Carl Roth \\
\hline Chlorhexidine & Biomol \\
\hline Ciprofloxacin & AppliChem \\
\hline DAPI (4',6-diamidino-2-phenylindole) & Carl Roth \\
\hline Dequalinium & Sigma-Aldrich \\
\hline Doxycycline & Fluka \\
\hline Ethidium bromide & Sigma-Aldrich \\
\hline Florfenicol & Biomol \\
\hline Kanamycin & Carl Roth \\
\hline Methy viologen & Sigma-Aldrich \\
\hline Minocycline & Sigma-Aldrich \\
\hline Mitomycin C & Biomol \\
\hline Norfloxacin & Sigma-Aldrich \\
\hline Tetracycline & Fluka \\
\hline Thiamphenicol & Sigma-Aldrich \\
\hline Tigecycline & Calbiochem \\
\hline Tetraphenylphosphonium chloride (TPP $\left.{ }^{+}\right)$ & Sigma-Aldrich \\
\hline
\end{tabular}




\subsubsection{Standards and kits}

\begin{tabular}{|l|l|}
\hline Standards and kits & Source \\
\hline GeneRuler 1kb DNA ladder & Thermo Fisher Scientific \\
\hline PageRuler protein ladder (SM0671) & Thermo Fisher Scientific \\
\hline Zyppy Plasmid Miniprep kit & Zymo Research \\
\hline DNA Clean \& Concentrator-5 kit & Zymo Research \\
\hline BCA kit & Thermo Fisher Scientific \\
\hline RNeasy Mini kit & Qiagen \\
\hline RNeasy MinElute Cleanup kit & Qiagen \\
\hline RNA clean \& concentrator-5 kit & Zymo Research \\
\hline QuantiNova SYBR Green RT-PCR kit & Qiagen \\
\hline Luna Universal One-Step RT-qPCR kit & New England Biolabs \\
\hline
\end{tabular}

\subsubsection{Bacteria strains}

\begin{tabular}{|c|c|c|}
\hline Name & Description & Source \\
\hline E. coli MACHT1 & $\begin{array}{l}F-\varphi 80(\text { lacZ) } \Delta M 15 \Delta l a c X 74 \text { hsdR(rK- } \\
m K+) \Delta r e c A 1398 \text { endA1 tonA }\end{array}$ & $\begin{array}{l}\text { Lab collection } \\
\text { (Themofisher) }\end{array}$ \\
\hline E. coli BW25113 $\Delta$ emrE $\Delta m d f A$ & $\begin{array}{l}F-\Delta(a r a D \text {-araB)567 } \Delta l a c Z 4787(: \because r r n B- \\
\text { 3) } \lambda \text { - rph-1 } \Delta(r h a D-r h a B) 568 \text { hsdR514 } \\
\Delta \text { emrE } \Delta m d f A\end{array}$ & $\begin{array}{l}\text { Lab collection } \\
\text { (Lytvynenko et al., } \\
\text { 2016) }\end{array}$ \\
\hline $\begin{array}{l}\text { E. coli BW25113 } \\
\Delta \text { emrE } \Delta m d f A \Delta a c r A B\end{array}$ & $\begin{array}{l}F-\Delta(a r a D \text {-araB)567 } \Delta l a c Z 4787(: \because r r n B- \\
\text { 3) } \lambda-r p h-1 \Delta(r h a D-r h a B) 568 \text { hsdR514 } \\
\Delta \text { emrE } \Delta m d f A \Delta a c r A B\end{array}$ & $\begin{array}{l}\text { Lab collection } \\
\text { (Lytvynenko et al., } \\
\text { 2016) }\end{array}$ \\
\hline A. baumannii ATCC 19606 & $\begin{array}{l}\text { Type strain from American Type Culture } \\
\text { Collection (ATCC) }\end{array}$ & $\begin{array}{l}\text { Prof Averhoff's lab } \\
\text { collection }\end{array}$ \\
\hline A. baumannii strain AYE & Epidemic strain in France & $\begin{array}{l}\text { Prof Averhoff's lab } \\
\text { collection }\end{array}$ \\
\hline $\begin{array}{l}\text { A. baumannii ATCC } 19606 \\
\Delta c r a A\end{array}$ & Markerless knockout of craA & This work \\
\hline $\begin{array}{l}\text { A. baumannii ATCC } 19606 \\
\triangle a d e A B\end{array}$ & Markerless knockout of ade $A B$ & This work \\
\hline $\begin{array}{l}\text { A. baumannii ATCC } 19606 \\
\Delta \text { adeFG }\end{array}$ & Markerless knockout of adeFG & This work \\
\hline $\begin{array}{l}\text { A. baumannii ATCC } 19606 \\
\Delta \text { adelJ }\end{array}$ & Markerless knockout of adelJ & This work \\
\hline
\end{tabular}




\subsubsection{Plasmids}

The plasmids used in this work are pTTQ18 (Stark, 1987) for functional assays in E. coli, a suicide plasmid pBIISK_sacB/kanR for production of markerless $A$. baumannii knockout strains and pBAV1K for complementation experiments in $A$. baumannii.

\subsubsection{Primers}

All oligonucleotides were obtained from Sigma-Aldrich.

Table 2.1 Primers used in Gibson assembly cloning method.

\begin{tabular}{|c|c|c|}
\hline Plasmid & Primer & Primer sequence (5'-3') \\
\hline $\begin{array}{l}\text { To amplify vector } \\
\text { backbone (pTTQ18) }\end{array}$ & $\begin{array}{l}\text { PTTQ18-F } \\
\text { PTTQ18-R }\end{array}$ & $\begin{array}{l}\text { catcaccatcatcatcatcattaataa } \\
\text { cgctgtttcctgtgtgaattgttatccgctc }\end{array}$ \\
\hline pTTQ18_MdfA & $\begin{array}{l}\operatorname{MdfA}-\mathrm{F} \\
\operatorname{MdfA}-\mathrm{R}\end{array}$ & $\begin{array}{l}\text { atttcacacaggaaacagcgatgcaaataaattagcttccggt } \\
\text { attaatgatgatgatgatggtgatgagtactagcccctcgtgaga }\end{array}$ \\
\hline pTTQ18_CraA_WT & $\begin{array}{l}\text { CraA-F } \\
\text { CraA-R }\end{array}$ & $\begin{array}{l}\text { atttcacacaggaaacagcgatgctgagacatagtggccgct } \\
\text { attaatgatgatgatgatggtgatgatgtaagtcttgtgcaactctttc }\end{array}$ \\
\hline PTTQ18_CmlA & $\begin{array}{l}\text { CmlA-F } \\
\text { CmlA-R }\end{array}$ & $\begin{array}{l}\text { atttcacacaggaaacagcgatgaccaccacacgccccg } \\
\text { attaatgatgatgatgatggtgatggacgactggcgacttctcg }\end{array}$ \\
\hline pTTQ18_Cm1A5 & $\begin{array}{l}\text { CmlA5-F } \\
\text { CmlA5-R }\end{array}$ & $\begin{array}{l}\text { atttcacacaggaaacagcgatgcgctcaaaaactttagttgg } \\
\text { attaatgatgatgatgatggtgatgacgattgggatttgatgtactttc }\end{array}$ \\
\hline PTTQ18_ABAYE0685 & $\begin{array}{l}0685-\mathrm{F} \\
0685-\mathrm{R}\end{array}$ & $\begin{array}{l}\text { atttcacacaggaaacagcgatgtctgaacaaacagcccag } \\
\text { attaatgatgatgatgatggtgatgagagagtgattggctttttgcttg }\end{array}$ \\
\hline PTTQ18_ABAYE0913 & $\begin{array}{l}0913-\mathrm{F} \\
0913-\mathrm{R}\end{array}$ & $\begin{array}{l}\text { atttcacacaggaaacagcgatgaagtcaaaatccatttccgtg } \\
\text { attaatgatgatgatgatggtgatgaacattcgattcttaatatctg }\end{array}$ \\
\hline pTTQ18_TetA & $\begin{array}{l}\text { Tet } A-F \\
\text { TetA-R }\end{array}$ & $\begin{array}{l}\text { atttcacacaggaaacagcgatgtccaccaacttatcagtgata } \\
\text { attaatgatgatgatgatggtgatggcgatcggctcgttgccctgc }\end{array}$ \\
\hline pTTQ18_TetG & $\begin{array}{l}\text { TetG-F } \\
\text { TetG-R }\end{array}$ & $\begin{array}{l}\text { atttcacacaggaaacagcgatgcgcagctctgccatcattg } \\
\text { attaatgatgatgatgatggtgatgcaatgaagttgcgaatggtctgc }\end{array}$ \\
\hline pTTQ18_TetAA & $\begin{array}{l}\text { TetAA-F } \\
\text { TetAA-R }\end{array}$ & $\begin{array}{l}\text { atttcacacaggaaacagcgatgcctgacttttcgataggacg } \\
\text { attaatgatgatgatgatggtgatgtctttgttgtacgactggtcgg }\end{array}$ \\
\hline $\begin{array}{l}\text { To amplify vector } \\
\text { backbone (pBAV1K) }\end{array}$ & $\begin{array}{l}\text { pBAV1K-F } \\
\text { pBAV1K-R }\end{array}$ & $\begin{array}{l}\text { ggcccgatcgatgccgccgcttaattaa } \\
\text { ctgaggcctgcagcggccgcgaa }\end{array}$ \\
\hline pBAV1K_CraA & $\begin{array}{l}\text { CraA_KO-F } \\
\text { CraA_KO-R }\end{array}$ & $\begin{array}{l}\text { cgcggccgctgcaggcctcaggctgattctgagtgcttgtacacc } \\
\text { gagttgcacaagacttacattaaggcccgatcgatgccgccgc }\end{array}$ \\
\hline $\begin{array}{l}\text { To amplify backbone } \\
\text { pBAV1K_TetA }\end{array}$ & $\begin{array}{l}\text { Tet } A-F \\
\text { TetA-R } \\
\text { TetR-F } \\
\text { TetR-R } \\
\text { Bk_tetA-F }\end{array}$ & $\begin{array}{l}\text { ggcctcagggcccgatcgatatgtccaccaacttatcagtgata } \\
\text { ggattaattaattaagcggcggctcagcgatcggctcgttgccctg } \\
\text { ggcctcagggccgatcgatatggctctgctgtagtgagt } \\
\text { tcactgataagttggtggacatattatgtttatcagtgataagtgtca } \\
\text { atgtccaccaacttatcagtgataaagatc }\end{array}$ \\
\hline
\end{tabular}


Table 2.2 Primers used for site-directed mutagenesis of CraA. The highlighted nucleotides represent the mutated residues.

\begin{tabular}{|c|c|}
\hline CraA variants & Primer sequence (5' to $\left.3^{\prime}\right)$ \\
\hline $\mathrm{E} 38 \mathrm{~A}$ & $\begin{array}{l}\text { Forward: TGCATTTGCGGTTTATATTGGTAA } \\
\text { Reverse: AATAGCACTAAAGCTAAAGGAAAC }\end{array}$ \\
\hline $\mathrm{E} 38 \mathrm{H}$ & $\begin{array}{l}\text { Forward: TCACTTTGCGGTTTATATTGGTAA } \\
\text { Reverse: same as E38A reverse primer }\end{array}$ \\
\hline E38Q & $\begin{array}{l}\text { Forward: TCAGTTTGCGGTTTATATTGGTAA } \\
\text { Reverse: same as E38A reverse primer }\end{array}$ \\
\hline E38D & $\begin{array}{l}\text { Forward: TGACTTTGCGGTTTATATTGGTAA } \\
\text { Reverse: same as E38A reverse primer }\end{array}$ \\
\hline $\mathrm{D} 46 \mathrm{~A}$ & $\begin{array}{l}\text { Forward: GGTAATGCCCTGATTCAGCC } \\
\text { Reverse: AATATAAACCGCAAATTCAAATAGC }\end{array}$ \\
\hline $\mathrm{D} 46 \mathrm{H}$ & $\begin{array}{l}\text { Forward: ATCACCTGATTCAGCCAGC } \\
\text { Reverse: TACCAATATAAACCGCAAATTC }\end{array}$ \\
\hline $\mathrm{D} 46 \mathrm{~N}$ & $\begin{array}{l}\text { Forward: ATAACCTGATTCAGCCAGC } \\
\text { Reverse: same as D46H primer }\end{array}$ \\
\hline $\mathrm{D} 46 \mathrm{E}$ & $\begin{array}{l}\text { Forward: ATGAACTGATTCAGCCAGC } \\
\text { Reverse: same as D46H primer }\end{array}$ \\
\hline E38A_D46A & Same primer pair as E38A (pTTQ18_CraA_D46A as template DNA) \\
\hline E38D_D46A & Same primer pair as E38D (pTTQ18_CraA_D46A as template DNA) \\
\hline E38A_D46E & Same primer pair as D46E (pTTQ18_CraA_E38A as template DNA) \\
\hline Y42A & $\begin{array}{l}\text { Forward: GTTGCTATTGGTAATGACCTG } \\
\text { Reverse: CGCAAATTCAAATAGCACTAAAGC }\end{array}$ \\
\hline N45A & $\begin{array}{l}\text { Forward: GCAGACCTGATTCAGCCAGC } \\
\text { Reverse: ACCAATATAAACCGCAAATTCAAA }\end{array}$ \\
\hline M70A & $\begin{array}{l}\text { Forward: CGCCGTCTTCAGCGTCATTT } \\
\text { Reverse: CCCAAGTTGCACTTACACCGAAAT }\end{array}$ \\
\hline Y73A & $\begin{array}{l}\text { Forward: TGCCTTATTAGGTGGTGCATC } \\
\text { Reverse: AATGACATTGAAGACGGCGC }\end{array}$ \\
\hline Y73F & $\begin{array}{l}\text { Forward: TTCTTATTAGGTGGTGCATCTG } \\
\text { Reverse: AAATGACATTGAAGACGGCGC }\end{array}$ \\
\hline Y73L & $\begin{array}{l}\text { Forward: CTGTTATTAGGTGGTGCATCTG } \\
\text { Reverse: same as Y73F reverse primer }\end{array}$ \\
\hline $\mathrm{L} 74 \mathrm{~A}$ & $\begin{array}{l}\text { Forward: GTCATTTTATGCATTAGGTGGTGC } \\
\text { Reverse: ATTGAAGACGGCGCCCAAGTT }\end{array}$ \\
\hline R124A & $\begin{array}{l}\text { Forward: CTGACATTAGCTTTTTTACAAGG } \\
\text { Reverse: AAAGTGTTCAATTTGTCGCGTTA }\end{array}$ \\
\hline $\mathrm{R} 124 \mathrm{H}$ & $\begin{array}{l}\text { Forward: CTGACATTACATTTTTTACAAGG } \\
\text { Reverse: same as R124A reverse primer }\end{array}$ \\
\hline L131A & $\begin{array}{l}\text { Forward: GGCAAGCGTAATTTCAGCAG } \\
\text { Reverse: CCAATACCTTGTAAAAAACGT }\end{array}$ \\
\hline S135A & $\begin{array}{l}\text { Forward: TTGCAGCAGTGGGATATGCC } \\
\text { Reverse: TTACGCTTAACCCAATACCTTG }\end{array}$ \\
\hline Y139A & $\begin{array}{l}\text { Forward: AACTTTGCCGAGCGTGATGCG } \\
\text { Reverse: GGAGCTGCCGCAATTCAGGAA }\end{array}$ \\
\hline
\end{tabular}


Materials and Methods

\begin{tabular}{|c|c|}
\hline M158A & $\begin{array}{l}\text { Forward: CCTCGCGGCAAATATTTCATTGC } \\
\text { Reverse: GCCATGACTTTAATCGCATCACG }\end{array}$ \\
\hline $\mathrm{S} 162 \mathrm{~A}$ & $\begin{array}{l}\text { Forward: ATTGCATTGCTTGCGCCTTTGC } \\
\text { Reverse: ATTTGCCATGAGGGCCATGAC }\end{array}$ \\
\hline L163A & $\begin{array}{l}\text { Forward: ATTTCAGCGCTTGCGCCTTTG } \\
\text { Reverse: same as S162A reverse primer }\end{array}$ \\
\hline P166A & $\begin{array}{l}\text { Forward: ATTGCTTGCGGCTTTGCTAG } \\
\text { Reverse: GAAATATTTGCCATGAGGGCC }\end{array}$ \\
\hline $\mathrm{L} 246 \mathrm{~A}$ & $\begin{array}{l}\text { Forward: GTATGCCGGCTATGCTTTGG } \\
\text { Reverse: CCACAAGTGGTAAGGCCAAC }\end{array}$ \\
\hline M2 $47 \mathrm{~A}$ & $\begin{array}{l}\text { Forward: GTATGCCGCTTGCGCTTTGG } \\
\text { Reverse: same as L } 246 \text { A reverse primer }\end{array}$ \\
\hline I250A & $\begin{array}{l}\text { Forward: GGGCTGCATTATCGCCAATTATT } \\
\text { Reverse: AAAGCATAAGCGGCATACCCAC }\end{array}$ \\
\hline Y268A & $\begin{array}{l}\text { Forward: AAGCTGGTTTAGCACAGTTCC } \\
\text { Reverse: GCACACTCGTTAGCTTCAGT }\end{array}$ \\
\hline F276A & $\begin{array}{l}\text { Forward: CCGGTAGCTTTAGGTTTAATTGT } \\
\text { Reverse: GAACTGTGCTAAACCATATTGCA }\end{array}$ \\
\hline L279A & $\begin{array}{l}\text { Forward: TTAGGTGCAATTGTTGGTAACAT } \\
\text { Reverse: TAGCACAGTTCCCGGTATTT }\end{array}$ \\
\hline N283A & $\begin{array}{l}\text { Forward: GTTTAATTGTTGGTGCCATTGTTT } \\
\text { Reverse: CTAAAAATACCGGGAACTGTGC }\end{array}$ \\
\hline E338A & $\begin{array}{l}\text { Forward: TTGGTGCAGGAATTAGCTTCTC } \\
\text { Reverse: AACAAATGAGTGTCATACCTATTAA }\end{array}$ \\
\hline F342A & $\begin{array}{l}\text { Forward: GAATTAGCGCCTCAGTGTTGTAC } \\
\text { Reverse: CTTCACCAAAACAAATGAGTGTCATA }\end{array}$ \\
\hline Y3 46A & $\begin{array}{l}\text { Forward: GCTCGTTTTGCGCTCATGTC } \\
\text { Reverse: CAACACTGAGAAGCTAATTCC }\end{array}$ \\
\hline R347A & $\begin{array}{l}\text { Forward: GTTGTACGCTTTTGCGCTCATG } \\
\text { Reverse: ACTGAGAAGCTAATTCCTTCACC }\end{array}$ \\
\hline K357A & $\begin{array}{l}\text { Forward: TGAAGTGTCAGCAGGAACTGTAG } \\
\text { Reverse: GATGACATGAGCGCAAAACGGTA }\end{array}$ \\
\hline L368A & $\begin{array}{l}\text { Forward: GCGATGACGAGCTTCTTTGC } \\
\text { Reverse: TAGCATTGAAACAGCAGCAGC }\end{array}$ \\
\hline M369A & $\begin{array}{l}\text { Forward: TTGGCGACGAGCTTCTTTGC } \\
\text { Reverse: same as L368A reverse primer }\end{array}$ \\
\hline F372A & $\begin{array}{l}\text { Forward: GCGCCTTTGCAATGATTG } \\
\text { Reverse: TCGTCATCAATAGCATTGAAAC }\end{array}$ \\
\hline
\end{tabular}


Table 2.3 Primers used for RT-qPCR with tigecycline-treated $A$. baumannii strain AYE.

\begin{tabular}{|l|l|}
\hline Gene & Primers (5' to $\mathbf{3}^{\prime}$ ) \\
\hline adeB & $\begin{array}{l}\text { Forward: gataaggcaccacaacaat } \\
\text { Reverse: tttcgcaatcagttgttcca }\end{array}$ \\
\hline adeG & $\begin{array}{l}\text { Forward: atcgcgtagtcaccagaacc } \\
\text { Reverse: cgtaactatgcggtgctcaa }\end{array}$ \\
\hline adeJ & $\begin{array}{l}\text { Forward: gaatggacgtatggttctcc } \\
\text { Reverse: cattgctttcatggcatcac }\end{array}$ \\
\hline tet $A$ & $\begin{array}{l}\text { Forward: gctcggtgggctgatgg } \\
\text { Reverse: ctttgtgcgactccggc }\end{array}$ \\
\hline rpoB & $\begin{array}{l}\text { Forward: gagtctaatggcggtggttc } \\
\text { Reverse: attgcttcatctgctggttg }\end{array}$ \\
\hline
\end{tabular}

Table 2.4 Primers used for construction of pBIISK_sacB/kanR_updown.

\begin{tabular}{|l|l|l|}
\hline \multicolumn{2}{|l}{ Cloning via restriction and ligation: } \\
\hline \multirow{4}{*}{ adeIJ knockout } & Primer & Primer sequence (5'-3') \\
\cline { 2 - 3 } & AdeIJ_up_for_NotI & ATTAGCGGCCGCCTTCCGTAAACGACATCAAGCTGC \\
\cline { 2 - 3 } & AdeIJ_up_rev_BamHI & ATGCGGATCCGTATGGTCTATTTCAGGTCGTAGC \\
\cline { 2 - 3 } & AdeIJ_down_for_BamHI & ATGCGGATCCCATCATTGTTCCACCTCGTTTAG \\
\cline { 2 - 3 } & AdeIJ_down_rev_PstI & ATTACTGCAGCGTCATTAGCCGTTCCGCATGCAGC \\
\hline \multirow{5}{*}{ CraA knockout } & AbeS_up_for_NotI & ATTAGCGGCCGCCCGATTGTAGCTATGCAACCAGACGC \\
\cline { 2 - 4 } & AbeS_up_rev_BamHI & CCGCGTCGAAGGGTACAGAATAGAAACCTAGGACGA \\
\cline { 2 - 3 } & AbeS_down_for_BamHI & GCGCGGATCCTTGCAGGCATTGTGATCATTAATGTG \\
\cline { 2 - 3 } & AbeS_down_rev_PstI & GGTTATCTTTCGTGATTATTGGTCCGGACGTCGCGC \\
\cline { 2 - 3 } & CraA_up_rev_BamHI & GCTGTCGCAACTTGGCATGCTGCACCTAGGATTA \\
\cline { 2 - 3 } & CraA_down_for_BamHI & ATTAGGATCCCAGGAAAGAGTTGCACAAGACTTAC \\
\cline { 2 - 3 } & CraA_down_rev_PstI & CATAAACCAATTCCGAGACACGTTGAGACGTCACGA \\
\hline
\end{tabular}

Table 2.4 to be continued in the next page. 
Materials and Methods

\begin{tabular}{|c|c|c|}
\hline \multicolumn{3}{|c|}{ Cloning via Gibson assembly method: } \\
\hline & Primer & Primer sequence $\left(5^{\prime}-3^{\prime}\right)$ \\
\hline \multirow{7}{*}{$\begin{array}{l}\text { ade } A B \\
\text { knockout }\end{array}$} & pBIISK_backbone_F & GAGCGGCCGCCACCGCGGTGG \\
\hline & pBIISK_backbone_R & TAGAACTAGTGGATCCCCCGG \\
\hline & Gib_adeAB_up_F & CGGGGGATCCACTAGTTCTAGGAGTTGCTGGTTCTCTACCAAGC \\
\hline & Gib_adeAB_up_R & CACCGCGGTGGCGGCCGCTCGCATACTGTCCAAACCTAGTGAG \\
\hline & Gib_adeAB_down_F & CTCACTAGGTTTGGACAGTATGCATCTCATCTTAAGTTCAATGCATC \\
\hline & Gib_adeAB_down_R & CACCGCGGTGGCGGCCGCTCCTTAAATCTCGTTGGACTCAGCTAT \\
\hline & Backbone_adeBup_R & GCATACTGTCCAAACCTAGTGAG \\
\hline \multirow{7}{*}{$\begin{array}{l}\text { adeFG } \\
\text { knockout }\end{array}$} & pBIISK_backbone_F & GAGCGGCCGCCACCGCGGTGG \\
\hline & pBIISK_backbone_R & TAGAACTAGTGGATCCCCCGG \\
\hline & Gib_adeFG_up_F & CGGGGGATCCACTAGTTCTACCTAAAGCACGGATTAAATTAATGC \\
\hline & Gib_adeFG_up_R & TCCACCGCGGTGGCGGCCGCTCGGAGGACATTTGGTGATTACATC \\
\hline & Gib_adeFG_down_F & GATGTAATCACCAAATGTCCTCCGACATGAGGTGCTCCTAGTTA \\
\hline & Gib_adeFG_down_R & TCCACCGCGGTGGCGGCCGCTCGCTTGGACATTCGTCGCAAC \\
\hline & Backbone_adeGup_R & GGAGGACATTTGGTGATTACATC \\
\hline
\end{tabular}

Table 2.5 Primers used for the first round and second round of colony-PCR.

\begin{tabular}{|c|c|c|}
\hline & Primer & Primer sequence (5'-3') \\
\hline \multirow[t]{4}{*}{ adeAB knockout } & adeAB_ctr_F & GTTAGGTCAAGTTCTGGTAC \\
\hline & adeAB_ctr_R & ATTGCGGAATGTACGGCCCT \\
\hline & adeAB_down_R & СTTAAATCTCGTTGGACTCAGCTA \\
\hline & adeAB_up_F & GGAGTTGCTGGTTCTCTACCAAGC \\
\hline \multirow[t]{4}{*}{ adeFG knockout } & adeFG_ctr_F & CGCTGACGTGATTGATAGC \\
\hline & adeFG_ctr_R & GCATTACCCAAATCAACATC \\
\hline & adeFG_down_R & GCTTGGACATTCGTCGCAAC \\
\hline & adeFG_up_F & CCTAAAGCACGGATTAAATTAA \\
\hline \multirow[t]{4}{*}{ adeIJ knockout } & AdeIJ_ctr_F & GTTGCATAGCCTCTTGGCGAGATTC \\
\hline & AdeIJ_ctr_R & GCTTGGCAGTTCTATCTTGATGAGC \\
\hline & AdeIJ_down_rev_PstI & ATTACTGCAGCGTCATTAGCCGTTCCGCATGCAGC \\
\hline & AdeIJ_up_for_NotI & ATTAGCGGCCGCCTTCCGTAAACGACATCAAGCTGC \\
\hline \multirow[t]{4}{*}{ craA knockout } & CraA_ctr_F & CCGGATTAGGCTGCTGCAAATAGTG \\
\hline & CraA_ctr_R & GACCCAGCTTATTGGCACGAAATC \\
\hline & CraA_down_rev_PstI & CATAAACCAATTCCGAGACACGTTGAGACGTCACGA \\
\hline & CraA_up_for_NotI & ATTAGCGGCCGCGCTTCCACATAACTATCTACATCAG \\
\hline
\end{tabular}


Table 2.6: Primers used for RT-qPCR with A. baumannii ATCC 19606 knockout strains.

\begin{tabular}{|l|l|}
\hline Gene & Primer sequence (5' to $\left.\mathbf{3}^{\prime}\right)$ \\
\hline adeB & $\begin{array}{l}\text { Forward: gaataaggcaccgcacaat } \\
\text { Reverse: tttcgcaatcagttgttcca }\end{array}$ \\
\hline adeG & $\begin{array}{l}\text { Forward: cgtaactatgcggtgctcaa } \\
\text { Reverse: atcgcgtagtcaccagaacc }\end{array}$ \\
\hline adeJ & $\begin{array}{l}\text { Forward: gcgaatggacgtatggttct } \\
\text { Reverse: cattgctttcatggcatcac }\end{array}$ \\
\hline craA & $\begin{array}{l}\text { Forward: atgctcactggtacgctaa } \\
\text { Reverse: gacacttcagatgacatgag }\end{array}$ \\
\hline abeS & $\begin{array}{l}\text { Forward: cgatcgggattgcctatgc } \\
\text { Reverse: caggcagccaagtctaatg }\end{array}$ \\
\hline ompA & $\begin{array}{l}\text { Forward: ttgctggcttaaacgtagttc } \\
\text { Reverse: ggtcttcagttaactcttgtg }\end{array}$ \\
\hline rpoB & $\begin{array}{l}\text { Forward: gagtctaatggcggtggttc } \\
\text { Reverse: attgcttcatctgctggttg }\end{array}$ \\
\hline
\end{tabular}

\subsubsection{Media}

\begin{tabular}{|l|l|}
\hline Medium & Compostition (for 1 L) \\
\hline $\begin{array}{l}\text { LB } \\
\text { (Lysogeny Broth) }\end{array}$ & $\begin{array}{l}10 \mathrm{~g} \text { tryptone, } 5 \mathrm{~g} \text { yeast extract, } 5 \mathrm{~g} \mathrm{NaCl} . \\
\text { For LB agar, } 16 \mathrm{~g} \text { of agar powder was added into 1 L of LB liquid } \\
\text { medium (autoclaved) }\end{array}$ \\
\hline Mueller-Hinton & $22 \mathrm{~g}$ of BLL ${ }^{\mathrm{TM}}$ Mueller Hinton II Broth (autoclaved) \\
\hline SOC & $\begin{array}{l}2 \% \text { tryptone, } 0.5 \% \text { yeast extract, } 10 \mathrm{mM} \mathrm{NaCl,} 2.5 \mathrm{mM} \mathrm{KCl} 10 \mathrm{mM} \\
\mathrm{MgCl}_{2}, 10 \mathrm{mM} \mathrm{MgSO}_{4}, 20 \mathrm{mM} \text { Glucose (sterilized } \mathrm{MgCl}_{2} \text { and glucose } \\
\text { were added to the autoclaved medium) }\end{array}$ \\
\hline
\end{tabular}




\subsection{Methods}

\subsubsection{Construction of knockout strains of A. baumannii ATCC 19606}

\subsubsection{Cloning of plasmid (pBIISK_sacB/kanR_updown)}

Construction of $A$. baumannii ATCC 19606 efflux pumps knockout strains ( $\triangle a d e A B, \triangle a d e F G$, $\triangle$ adelJ and $\triangle c r a A$ ) was performed as described in Stahl et al. 2015. In brief, the up- and downstream regions of the gene of interest (approximately $1500 \mathrm{bp}$ each) were amplified from the genomic DNA of $A$. baumannii ATCC 19606 with the PCR ingredients listed in Table 2.7 and PCR programme in Table 2.8. Agarose gel electrophoresis was carried out to check the PCR products. The insertion of the upstream and downstream regions into the vector pBIISK_sacB/kanR were carried out either via restriction digestion using the Pstl, BamHI and Notl restriction sites or via Gibson assembly (Gibson et al., 2009) (as described in Appendix Method, section 5.1.1). The primers used were listed in Table 2.4. Transformation of $E$. coli MACHT1 and plasmid minipreparation was conducted according to Appendix Method, section 5.1.3 to 5.1.5. The constructs were verified by sequencing (Eurofins).

Table 2.7 PCR ingredients for amplification of the upstream and downstream regions.

\begin{tabular}{|l|c|}
\hline Chemicals & Volume $(\boldsymbol{\mu L})$ \\
\hline $5 x$ GC buffer & 10.0 \\
\hline Forward primer (Stock: $10 \mathrm{p} \mathrm{mol})$ & 1.2 \\
\hline Reverse primer (Stock: $10 \mathrm{p} \mathrm{mol})$ & 1.2 \\
\hline Genomic DNA of $A$. baumannii ATCC $19606($ Stock: $100 \mathrm{ng} / \mu \mathrm{l})$ & 0.5 \\
\hline dNTPs $(10 \mathrm{mM})$ & 1.5 \\
\hline ddH $_{2} \mathrm{O}$ & 34.6 \\
\hline Phusion high-fidelity DNA polymerase $(2 \mathrm{U} / \mu \mathrm{L})$ & 1.0 \\
\hline Total volume & 50.0 \\
\hline
\end{tabular}


Table 2.8 PCR programme for amplification of upstream and downstream regions.

\begin{tabular}{|l|l|l|l|}
\hline Steps & Temperature & Time & Cycle \\
\hline Initial denaturation & $98^{\circ} \mathrm{C}$ & 30 seconds & 1 \\
\hline Denaturation & $98^{\circ} \mathrm{C}$ & 10 seconds & \\
\hline Annealing & $58^{\circ} \mathrm{C}$ & 15 seconds & 30 \\
\hline Extension & $72^{\circ} \mathrm{C}$ & 60 seconds & \\
\hline Final extension & $72^{\circ} \mathrm{C}$ & 10 minutes & 1 \\
\hline
\end{tabular}

\subsubsection{Transformation and the first round of colony-PCR}

The plasmid pBIISK_sacB/kanR_updown was transformed into electrocompetent $A$. baumannii ATCC19606 cells by electroporation (as described in Appendix Method, section 5.1.6 and 5.1.7). Integration of the plasmid was monitored by colony-PCR using two primer pairs [(ctr_F and down_R) and (up_F and ctr_R)] as listed in Table 2.5. Each of the selected single colony was suspended in $50 \mu \mathrm{L}$ of $\mathrm{ddH}_{2} \mathrm{O}$, heated at $95^{\circ} \mathrm{C}$ for 20 min and served as template for the colony-PCR. The PCR ingredients and programme were shown in Table 2.9 and Table 2.10, respectively.

Table 2.9 Ingredients for the colony-PCR.

\begin{tabular}{|l|c|}
\hline Master mix & Volume $(\mu \mathrm{L})$ \\
\hline GC buffer & 20.0 \\
\hline dNTP $(2 \mathrm{mM})$ & 8.0 \\
\hline Primer I $(10 \mu \mathrm{M})$ & 1.0 \\
\hline Primer II $(10 \mu \mathrm{M})$ & 1.0 \\
\hline Phusion & 1.0 \\
\hline dd $_{2} \mathrm{O}$ & 68.0 \\
\hline Total volume & 100.0 \\
\hline Each reaction: Master mix $(20 \mu \mathrm{L})+$ DNA $(2 \mu \mathrm{L})$ \\
\hline
\end{tabular}


Table 2.10 PCR programme for colony-PCR.

\begin{tabular}{|l|l|l|l|}
\hline Steps & Temperature & Time & Cycle \\
\hline Initial denaturation & $98^{\circ} \mathrm{C}$ & 5 minutes & 1 \\
\hline Denaturation & $98^{\circ} \mathrm{C}$ & 30 seconds & \\
\hline Annealing & $60^{\circ} \mathrm{C}$ & $\begin{array}{l}30 \text { seconds } \\
30 \text { seconds } / 1 \mathrm{~kb}\end{array}$ & 30 \\
\hline Extension & $72^{\circ} \mathrm{C}$ & 10 minutes & 1 \\
\hline Final extension & $72^{\circ} \mathrm{C}$ & & \\
\hline
\end{tabular}

\subsubsection{Counter-selection using sucrose and second round of colony-PCR}

The correct integrants were subjected to the first round of counter-selection with LB liquid medium supplemented with $10 \%$ sucrose and subsequently to the second round of counterselection on LB agar supplemented with $10 \%$ sucrose. Colonies were tested for kanamycin sensitivity by replica plating onto LB agar without kanamycin and LB agar supplemented with $50 \mu \mathrm{g} / \mathrm{mL}$ kanamycin. Kanamycin-sensitive colonies were subjected to the second round of colony-PCR (as described in 2.2.1.2) using primers ctr_F and ctr_R as listed in Table 2.5. Positive knockout strains were further confirmed by sequencing (Eurofin).

\subsubsection{Differential gene expression analysis for $\boldsymbol{A}$. baumannii knockout strains}

\subsubsection{Sample preparation and RNA extraction}

Before sample preparion, MIC measurement was conducted as described in 2.2.6.3 using LB medium to determine a suitable subinhibitory concentration of chloramphenicol for each A. baumannii ATCC 19606 strain. Overnight cultures of $A$. baumannii ATCC 19606 wild-type, $\triangle c r a A, \triangle a d e A B$ or $\triangle a d e l J$ were inoculated into $50 \mathrm{~mL}$ of LB medium supplemented with subinhibitory concentration of chloramphenicol to an initial $\mathrm{OD}_{600}$ of 0.05 , incubated at $37^{\circ} \mathrm{C}$, $130 \mathrm{rpm}$ to an $\mathrm{OD}_{600}$ of 0.5 to 0.7 . For untreated samples (control), cells were grown in LB medium without chloramphenicol to the same growth phase. Cell suspension of $500 \mu \mathrm{L}$ with $\mathrm{OD}_{600}$ of 0.6 was mixed with two volumes of RNAprotect bacteria reagent (Qiagen) and incubated at room temperature for 5 minutes. After centrifugation at $8000 \times \mathrm{g}$ for 10 minutes, the cell pellet was kept at $-80^{\circ} \mathrm{C}$ for further use. 
RNA extraction was conducted using RNeasy Mini Kit (Qiagen). DNA was removed by oncolumn DNA digestion (Qiagen) and Turbo DNase (Invitrogen). RNA samples were purified by RNeasy MinElute Cleanup kit (Qiagen). The concentration and the quality of the RNA samples were checked with Nanodrop.

\subsubsection{Quantitative reverse transcription PCR}

One-step RT-qPCR was conducted on a thermal cycler Rotor-Gene Q (Qiagen) using QuantiNova SYBR Green RT-PCR kit (Qiagen). The reaction mixture is listed in Table 2.11 and the PCR programme was set according to Table 2.12. The expression of gene of interests was quantified using the primers listed in Table 2.6, with rpoB as reference gene.

Table 2.11 Reaction mixture for RT-qPCR using QuantiNova SYBR Green RT-PCR kit.

\begin{tabular}{|l|c|}
\hline Chemicals & Volume $(\boldsymbol{\mu L})$ \\
\hline 2x SYBR Green RT-PCR master mix & 5.0 \\
\hline QN SYBR Green RT-Mix $(100 x)$ & 0.1 \\
\hline Forward primer $(10 \mu \mathrm{M})$ & 0.5 \\
\hline Reverse primer $(10 \mu \mathrm{M})$ & 0.5 \\
\hline Template RNA $(10 \mu \mathrm{g} / \mathrm{mL})$ & 2.0 \\
\hline Nuclease-free water & 1.9 \\
\hline Total volume & 10.0 \\
\hline
\end{tabular}

Table 2.12 PCR programme for RT-qPCR using QuantiNova SYBR Green RT-PCR kit.

\begin{tabular}{|l|l|l|l|}
\hline Steps & Temperature & Time & Cycle \\
\hline Reverse transcription & $50^{\circ} \mathrm{C}$ & 12 minutes & 1 \\
\hline $\begin{array}{l}\text { PCR initial heat } \\
\text { activation }\end{array}$ & $95^{\circ} \mathrm{C}$ & 2 minutes & 1 \\
\hline Denaturation & $95^{\circ} \mathrm{C}$ & 5 seconds & \multirow{2}{*}{40} \\
\hline $\begin{array}{l}\text { Combined } \\
\text { annealing/extension }\end{array}$ & $60^{\circ} \mathrm{C}$ & 10 seconds & 1 \\
\hline melt curve analysis & $65^{\circ} \mathrm{C}-95^{\circ} \mathrm{C}$ & 5 seconds for each ${ }^{\circ} \mathrm{C}$ & 1 \\
\hline
\end{tabular}




\subsubsection{Cloning of putative chloramphenicol or tetracycline transporter genes}

Five putative chloramphenicol transporter genes (CraA: 12_508; CmIA: ABAYE3640 ; CmIA5: ABAYE3620 ; ABAYE0685 ; ABAYE0913) and three putative tetracycline transporter genes (TetA: ABAYE3597 ; TetG: ABAYE3637 ; TetA(A): ABAYE0369) were amplified from chromosomal DNA of $A$. baumannii strain AYE and inserted onto PTTQ18 vector via Gibson assembly method. A fusion tag consists of six histidines was attached to the C-terminal of the protein for Western blot detection. For complementation assay in A. baumannii ATCC 19606, pBAV1K_CraA containing craA gene and 700 bp upstream region and pBAV1K_TetA containing tetA gene and 727 bp upstream region were constructed via similar method using chromosomal DNA of $A$. baumannii ATCC 19606. The primers used were listed in Table 2.1.

\subsubsection{PCR based site-directed mutagenesis of CraA}

In an inverse PCR, the primer pair located back-to-back, with one of the primer contains the desired mutation (Primers are listed in Table 2.2). The primers were phosphorylated prior to PCR, as the 5' end of the linear PCR product should contain phosphate group in order to be ligated and form circularized plasmid. The reaction mixture (Table 2.13) was incubated at $37^{\circ} \mathrm{C}$ for 30 minutes, followed by enzyme inactivation at $70^{\circ} \mathrm{C}$ for 10 minutes. PCR was conducted using the reaction mix and programme as shown in Table 2.14 and Table 2.15, respectively. The PCR products were analyzed by agarose gel electrophoresis. The samples and $1 \mathrm{~kb}$ DNA marker were loaded onto $1 \%$ agarose gel. Electrophoresis was carried out at $120 \mathrm{~V}$ for 60 minutes and the gel was visualized under UV light.

Table 2.13 Chemicals for phosphorylation of primers.

\begin{tabular}{|l|c|}
\hline Chemicals & Volume $(\boldsymbol{\mu L})$ \\
\hline Primer (Stock: $100 \mathrm{p} \mathrm{mol})$ & 1.0 \\
\hline 10x T4 PNK A Buffer & 2.0 \\
\hline ATP (Stock: $5 \mathrm{mM})$ & 2.0 \\
\hline T4 polynucleotide kinase (Stock: $10 \mathrm{U} / \mu \mathrm{l})$ & 1.0 \\
\hline $\mathrm{ddd}_{2} \mathrm{O}$ & 14.0 \\
\hline Total volume & 20.0 \\
\hline
\end{tabular}


Table 2.14 Chemicals for inverse PCR.

\begin{tabular}{|l|c|}
\hline Chemicals & Volume $(\boldsymbol{\mu L})$ \\
\hline 2x Phusion Flash Mix & 10.0 \\
\hline Forward primer (phosphorylated) (Stock: $5 \mathrm{p} \mathrm{mol})$ & 1.2 \\
\hline Reverse primer (phosphorylated) (Stock: $5 \mathrm{p} \mathrm{mol})$ & 1.2 \\
\hline Template DNA (pTTQ18) (Stock: $20 \mathrm{ng} / \mu \mathrm{l})$ & 0.2 \\
\hline $\mathrm{ddH}_{2} \mathrm{O}$ & 7.4 \\
\hline Total volume & 20.0 \\
\hline
\end{tabular}

Table 2.15 PCR programme.

\begin{tabular}{|l|l|l|l|}
\hline Steps & Temperature & Time & Cycle \\
\hline $\begin{array}{l}\text { Initial } \\
\text { denaturation }\end{array}$ & $98^{\circ} \mathrm{C}$ & 30 seconds & 1 \\
\hline Denaturation & $98^{\circ} \mathrm{C}$ & 10 seconds & \\
\cline { 1 - 2 } Annealing & $58^{\circ} \mathrm{C}$ & 15 seconds & 30 \\
\cline { 1 - 3 } Extension & $72^{\circ} \mathrm{C}$ & 3 minutes & \\
\hline Final extension & $72^{\circ} \mathrm{C}$ & 5 minutes & 1 \\
\hline
\end{tabular}

To remove the template plasmid DNA, restriction digestion with Dpnl which cuts only the methylated DNA was conducted. The newly synthesized mutated PCR product is not methylated and therefore is not affected. The reaction mixture (Table 2.16) was incubated at $37^{\circ} \mathrm{C}$ for 2 hours. The purification of PCR product was performed using DNA Clean \& Concentrator-5 kit (Zymo Research). The two ends of the linear PCR product were joined together to form circular plasmid through ligation. The ligation reaction (Table 2.17) was incubated at room temperature overnight. For plasmid amplification, E. coli MACHT1 was transformed with $2 \mu \mathrm{L}$ of ligation product according to the method described in Appendix Method, section 5.1.4. Plasmid minipreparation was performed according to Appendix Method, section 5.1.5. The mutations were verified by sequencing (Eurofins). 
Table 2.16 Chemicals for Dpnl digestion.

\begin{tabular}{|l|c|}
\hline Chemicals & Volume $(\boldsymbol{\mu L})$ \\
\hline PCR product & 16.0 \\
\hline $10 x$ Fast Digest Buffer & 3.0 \\
\hline$D p n l$ & 1.0 \\
\hline $\mathrm{ddH}_{2} \mathrm{O}$ & 10.0 \\
\hline Total volume & 30.0 \\
\hline
\end{tabular}

Table 2.17 Chemicals for ligation reaction.

\begin{tabular}{|l|c|}
\hline Chemicals & Volume $(\boldsymbol{\mu L})$ \\
\hline Purified PCR product & 11.0 \\
\hline T4 DNA ligase & 1.0 \\
\hline 10x T4 DNA Ligase buffer & 1.5 .0 \\
\hline $50 \%$ PEG4000 & 1.5 \\
\hline Total volume & 15.0 \\
\hline
\end{tabular}

\subsubsection{Characterization of CraA of $A$. baumannii strain AYE}

\subsubsection{Drug susceptibility assays in E. coli}

The preparation of competent cells of E. coli BW25113 $\Delta$ emrEsmdfA or E. coli BW25113 $\triangle e m r E \Delta m d f A \triangle a c r A B$ and the transformation were conducted according to the method described in Appendix Method, section 5.1.3 and 5.1.4. Single colony of E. coli BW25113

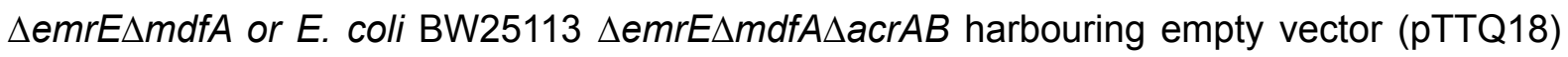
or pTTQ18 containing putative chloramphenicol transporters (as listed in Table 2.1) were grown overnight in $5 \mathrm{~mL}$ of LB medium containing $50 \mu \mathrm{g} / \mathrm{mL}$ carbenicillin or $100 \mu \mathrm{g} / \mathrm{mL}$ ampicillin at $37^{\circ} \mathrm{C}$ at $180 \mathrm{rpm}$. Cells were adjusted to $\mathrm{OD}_{600}$ of approximately 1.0 , followed by a serial dilution up to $10^{-5}$. Four $\mu \mathrm{L}$ of each diluted cultures were spotted on an LB agar plate containing $50 \mu \mathrm{g} / \mathrm{mL}$ carbenicillin or $100 \mu \mathrm{g} / \mathrm{mL}$ ampicillin, $0.2 \mathrm{mM}$ IPTG and selected drugs. Drug agar plates were incubated for 18 to 20 hours at $37^{\circ} \mathrm{C}$. Pictures of plates were taken using ImageQuant LAS4000. 
The growth of cells was scored based on the number of dilution that exhibits cell growth, i.e. variant that grow to the last dilution was scored as 6 . A relative growth of variant in comparison to the WT on the same drug agar plate was calculated (Table 2.8). Results from at least three biological replicates were averaged and presented with standard error of mean (s.e.m). Student's T-test was conducted to determine the significance of difference. For example the relative growth for Variant A shown in Table 2.18 is $0.475 \pm 0.025(p<0.005)$.

Table 2.18 An example of scoring and relative growth determination for CraA variants.

\begin{tabular}{|l|c|c|c|c|}
\hline & \multicolumn{4}{|c|}{ Scoring } \\
\hline Cells & Replicate 1 & Replicate 2 & Replicate 3 & Replicate 4 \\
\hline WT & 6 & 5 & 6 & 6 \\
\hline Variant A & 3 & 2 & 3 & 3 \\
\hline
\end{tabular}

\begin{tabular}{|c|c|c|c|c|c|c|c|}
\hline & \multicolumn{4}{|c|}{ Relative growth } & Average & s.e.m & $P$ value \\
\hline Cells & Replicate 1 & Replicate 2 & Replicate 3 & Replicate 4 & & & \\
\hline WT & 1 & 1 & 1 & 1 & 1 & 0 & \\
\hline Variant A & 0.5 & 0.4 & 0.5 & 0.5 & 0.475 & 0.025 & $5.69 \times 10^{-8}$ \\
\hline
\end{tabular}

\subsubsection{Sample preparation for Western blot analysis}

The gene expression of $c r a A$ and $c r a A$ variants was determined from the protein detection by Western blot analysis. The sample preparation was conducted according to Ma et al. 2013 with slight modification. The cell pellets obtained from the agar plate were resuspended in $500 \mu \mathrm{L}$ of $0.2 \mathrm{M}$ Tris $/ \mathrm{HCl} \mathrm{pH8}$ and incubated at room temperature for 20 minutes. To lyse the cells, $243 \mu \mathrm{L}$ of Lysis Buffer $(0.2 \mathrm{M}$ Tris/ $\mathrm{HCl}$ pH8, $1 \mathrm{M}$ sucrose, $1 \mathrm{mM}$ EDTA) was added to the cell suspension. After 90 seconds of incubation, $3.3 \mu \mathrm{L}$ of lysozyme $10 \mathrm{mg} / \mathrm{mL}$ was added to the cell suspension and the mixture is incubated at room temperature for another 30 seconds. Subsequently, $480 \mu \mathrm{L}$ of water was added to the mixture and incubated at room temperature for 20 minutes at $700 \mathrm{rpm}$. Then, the sample was centrifuged at $15000 \mathrm{x} \mathrm{g}$ for 20 minutes. The pellet was resuspended in $1 \mathrm{~mL}$ water and incubated at room temperature for 20 minutes at $700 \mathrm{rpm}$. The membranes were sedimented at $15000 \mathrm{x} \mathrm{g}$ for 20 minutes. For each of the $10 \mathrm{mg}$ pelleted membrane, $25 \mu \mathrm{L}$ of Suspension Buffer $(10 \mathrm{mM}$ Tris- $\mathrm{HCl}$ 
$\mathrm{pH} 8,5 \%$ glycerol, $1 \mathrm{mM} ß$-mercaptoethanol) was added. The membrane suspension was subjected to SDS-PAGE and Western blot as described in Appendix Method, section 5.1.2.

\subsubsection{Dequalinium transport assay in whole cells}

The experiment was performed according to Fluman et al. 2014 with slight modification. Single colony of $E$. coli BW25113 $\triangle$ mdfA $\triangle$ emrE $\triangle a c r A B$ cells harbouring empty vector (pTTQ18) or plasmid pTTQ18_CraA_WT or CraA variants were inoculated into $5 \mathrm{~mL}$ LB liquid medium supplemented with $100 \mu \mathrm{g} / \mathrm{mL}$ ampicillin and grown overnight at $37^{\circ} \mathrm{C}$. Precultures were inoculated into fresh $20 \mathrm{~mL}$ LB liquid medium supplemented with $100 \mu \mathrm{g} / \mathrm{mL}$ ampicillin and grew until $\mathrm{OD}_{600}$ of approximately 0.6. The cells were induced with $0.2 \mathrm{mM}$ IPTG (final concentration) and grown for another three hours at $37^{\circ} \mathrm{C}$. Cell suspension $(1 \mathrm{~mL}$ of $\mathrm{OD}_{600}$ of 1.0) was pelleted, washed once with Wash Buffer $(50 \mathrm{mM} \mathrm{KPi} \mathrm{pH} 7 ; 0.2 \%$ glucose; $2 \mathrm{mM} \mathrm{MgSO}_{4}$ ) and resuspended in $100 \mu \mathrm{L}$ of the same buffer. Subsequently, $50 \mu \mathrm{L}$ of the cell suspension was mixed with $950 \mu \mathrm{L}$ of pre-warmed buffer supplemented with $2 \mu \mathrm{M}$ dequalinium, then $200 \mu \mathrm{L}$ from the mixture was transferred to 96-well plate for dequalinium fluorescence measurement (excitation and emission at 330 and $370 \mathrm{~nm}$, respectively) using Tecan Microplate Reader. For the experiment with chloramphenicol as a competitor, the prewarmed buffer was supplemented with $1 \mathrm{mM}$ chloramphenicol in addition of dequalinium. To determine the amount of dequalinium trapped inside the cells, the cells were incubated with Wash Buffer supplemented with $2 \mu \mathrm{M}$ dequalinium for 0 or 15 minutes at $37^{\circ} \mathrm{C}$. Lysis of the cells was performed with $1 \%$ SDS and the dequalinium fluorescence in the cell lysate was determined.

\subsubsection{Ethidium efflux in whole cells}

E. coli BW25113 $\triangle$ emrEAmdfA cells harbouring empty vector PTTQ18 or plasmids encoding wild-type CraA or CraA variants were grown in $20 \mathrm{~mL}$ LB liquid medium at $37^{\circ} \mathrm{C}$ until cell cultures reached an $\mathrm{OD}_{600}$ of 0.5 to 0.6 . Cells were induced with $0.2 \mathrm{mM}$ IPTG for 2 hours before they were harvested, washed twice with Dulbecco's Phosphate Buffered Saline (DPBS) $\left(8 \mathrm{mg} / \mathrm{mL} ; 0.2 \mathrm{mg} / \mathrm{mL} \mathrm{KCl} ; 1.44 \mathrm{mg} / \mathrm{mL} \mathrm{Na}_{2} \mathrm{HPO}_{4} .2 \mathrm{H}_{2} \mathrm{O} ; 0.2 \mathrm{mg} / \mathrm{mL} \mathrm{KH}_{2} \mathrm{PO}_{4} ; 10 \mathrm{mM}\right.$ $\mathrm{MgCl}_{2}$ ) and resuspended in the same buffer to $\mathrm{OD}_{600}$ of 1.0 (one $\mathrm{mL}$ ). To abolish the proton 
gradient, $40 \mu \mathrm{M}$ of carbonyl cyanide m-chlorophenyl hydrazone (CCCP) was added and incubated at $37^{\circ} \mathrm{C}$ for $10 \mathrm{~min}$, followed by $10 \mu \mathrm{M}$ of ethidium bromide for $45 \mathrm{~min}$. Cultures were harvested by centrifugation at $8000 \times \mathrm{g}$, washed once with DPBS and resuspended with the same buffer. Next, $200 \mu \mathrm{L}$ of the cell suspension was transferred to 96 -well plate. To initiate efflux, $0.36 \%$ glucose was added and the fluorescence was measured immediately (excitation and emission wavelengths of 535 and $610 \mathrm{~nm}$, respectively) at $25^{\circ} \mathrm{C}$ using a Tecan Microplate Reader.

\subsubsection{Norfloxacin accumulation assay in whole cells}

The experiment was conducted according to Mortimer and Piddock, 1991 with modification. E. coli BW25113 $\Delta$ emrE $\Delta m d f A$ cells harbouring empty vector PTTQ18 or plasmids encoding wild-type CraA or mutants were grown in LB liquid medium at $37^{\circ} \mathrm{C}$ until cell cultures reached an $\mathrm{OD}_{600}$ of 0.5 to 0.6 . Cells were induced with $0.2 \mathrm{mM}$ IPTG for 2 hours before they were harvested, washed twice with DPBS and resuspended in the same buffer to an $\mathrm{OD}_{600}$ of 1.0 (one $\mathrm{mL}$ ). The cells were incubated with norfloxacin (final concentration: $100 \mu \mathrm{g} / \mathrm{mL}$ ) for 5 minutes at room temperature. The cells were harvested with centrifugation $(7500 \mathrm{~g}$, 90s) and washed twice with DPBS, followed by cell lysis using $800 \mu \mathrm{L}$ of $1 \%$ SDS in $0.1 \mathrm{M}$ glycine $\mathrm{HCl}(\mathrm{pH} 3)$ for 10 minutes at room temperature. The cell lysate was subjected to centrifugation at $13000 \mathrm{~g}$ for 5 minutes, before $180 \mu \mathrm{L}$ of the supernatant was transferred to 96-well plate for fluorescence measurement using a Tecan Microplate Reader (excitation and emission wavelengths of 281 and $440 \mathrm{~nm}$, respectively).

\subsubsection{Proton transport assay in inverted membrane vesicles}

The experiment was conducted according to Fluman et al. 2014 with slight modification. Single colony of $E$. coli BW25113 $\triangle m d f A \Delta e m r E \Delta a c r A B$ cells overexpressing $c r a A$ or craA variants or harbouring empty vector (pTTQ18) were inoculated into $100 \mathrm{~mL}$ LB medium supplemented with $100 \mu \mathrm{g} / \mathrm{mL}$ ampicillin and grown overnight at $37^{\circ} \mathrm{C}, 180 \mathrm{rpm}$. Precultures were inoculated into $1 \mathrm{~L}$ of LB liquid medium supplemented with $100 \mu \mathrm{g} / \mathrm{mL}$ ampicillin. The cells were grown to $\mathrm{OD}_{600}$ of approximately 0.6 , induced with $0.2 \mathrm{mM}$ IPTG (final concentration) for 3 hours and harvested with centrifugation at $6000 \mathrm{x} \mathrm{g}$ for 15 minutes. Cell pellets were washed once and suspended in Membrane Suspension Buffer (50 mM KPi 
$\mathrm{pH} 7.3 ; 10 \%$ glycerol; $5 \mathrm{mM} \mathrm{MgSO}_{4}$ ). Lysis of the cells was conducted using Stansted high pressure homogenizer, followed by centrifugation at $25000 \mathrm{xg}$ for 20 minutes to remove the cell lysates. The supernatant fraction was subjected to ultracentrifugation $\left(120000 \times \mathrm{g}, 4^{\circ} \mathrm{C}\right)$ for $150 \mathrm{~min}$ to collect the membranes. The membranes were resuspended with the same buffer. The protein concentration was measured with BCA assay and adjusted to $20 \mathrm{mg} / \mathrm{mL}$. Aliquots were frozen in liquid nitrogen and stored at $-80^{\circ} \mathrm{C}$.

To start the proton transport assay, $20 \mu \mathrm{l}$ of thawed membranes were added into one $\mathrm{mL}$ of pre-warmed $\left(30^{\circ} \mathrm{C}\right)$ Proton Transport Buffer $(50 \mathrm{mM}$ potassium gluconate; $50 \mathrm{mM} \mathrm{KCl} ; 10$ $\mathrm{mM} \mathrm{MgSO}_{4}$; titrated to $\mathrm{pH} 6$ and supplemented with $1 \mu \mathrm{M} \mathrm{ACMA}$ ) and incubated at $30^{\circ} \mathrm{C}$ for 5 min. Fluorescence measurement (excitation and emission wavelengths of 409 and 474 , respectively) was performed in 96-well plate using a Tecan Microplate Reader. After one minute of measurement, DL-lactate (final concentration: $2 \mathrm{mM}$ ) was added, followed by the tested drug and CCCP (final concentration: $0.01 \mathrm{mM}$ ) at indicated time points.

\subsubsection{Complementation of CraA to A. baumannii ATCC $19606 \Delta c r a A$}

The preparation of electrocompetent $A$. baumannii ATCC 19606 wild-type and A. baumannii ATCC $19606 \triangle c r a A$ were conducted as described in Appendix Method, section 5.1.6. Electroporation was carried out according to Appendix Method, section 5.1.7 to introduce the empty vector $(\mathrm{pBAV} 1 \mathrm{~K})$ or complementation plasmid (pBAV1K_CraA) into the cells. To determine the drug resistant phenotype of the complemented strains, drug agar plate assay was conducted as described in 2.2.5.1 using LB agar plate supplemented with $50 \mu \mathrm{g} / \mathrm{mL}$ kanamycin and tested drugs. 


\subsubsection{Molecular docking of CraA}

The model of CraA was created via the protein structure and function prediction method ITASSER (Iterative Threading ASSEmbly Refinement) (Yang et al., 2015), based on the MdfA-Q131R_L339E structure (PDB: 6EUQ) (Zomot et al., 2018). Protein and ligand (chloramphenicol, ethidium, and norfloxacin) input files were prepared with AutoDock Tools (Morris et al., 2009). Molecular docking calculations were performed with AutoDock Vina (Trott and Olson, 2010). The search poses were performed within a central cavity of CraA model with a search space of $24 \times 24 \times 24 \AA^{3}$ and exhaustiveness of 120 .

\subsubsection{Characterization of TetA of A. baumannii strain AYE}

\subsubsection{Drug susceptibility assays in E. coli}

The drug susceptibility assays using E. coli BW25113 $\Delta$ emrEAmdfA or E. coli BW25113 $\triangle e m r E \Delta m d f A \triangle a c r A B$ harbouring empty vector (pTTQ18) or plasmid containing putative transporters pTTQ18_TetA, pTTQ18_TetG or pTTQ18_TetA(A) were conducted as described in 2.2.5.1. Serial diluted cell cultures were spotted on an LB agar plate containing $50 \mu \mathrm{g} / \mathrm{mL}$ carbenicillin, 0.1 mM IPTG and selected tetracyclines.

\subsubsection{MIC determination of tetracyclines in $E$. coli overexpressing tetA or tetG}

As the $E$. coli overexpressing $\operatorname{tet} A(A)$ did not grow well on the control plate without tested drugs, it was excluded from the subsequent experiments. Minimal inhibitory concentrations (MICs) of four tetracyclines were determined with microdilution method. Overnight cultures of E. coli BW25113 $\Delta$ emrE $\Delta m d f A$ or E. coli BW25113 $\Delta$ emrE $\Delta m d f A \Delta a c r A B$ harbouring empty vector (pTTQ18), pTTQ18_TetA or pTTQ18_TetG were diluted to OD $_{600}$ of 0.02 . Cell cultures of $75 \mu \mathrm{l}$ were inoculated into $75 \mu \mathrm{l}$ of serial 2-fold substrate dilutions in freshly prepared Mueller-Hinton Broth supplemented with $50 \mu \mathrm{g} / \mathrm{mL}$ carbenicillin and $0.1 \mathrm{mM}$ IPTG in 96-well plates. The plates were incubated at $37^{\circ} \mathrm{C}$ for 20 hours with $900 \mathrm{rpm}$. Experiments were conducted with $n \geq 4$. 


\subsubsection{Tigecycline susceptibility assays in A. baumannii}

Tigecycline susceptibility of the $A$. baumannii ATCC 19606 wild-type and RND knockout strains ( $\triangle$ adeAB, $\triangle$ adeFG, and $\triangle$ adelJ) harbouring empty vector (pBAV1K) or pBAV1K_TetA was determined via MIC measurement and drug agar plate assays. MIC measurements were conducted by inoculating $25 \mu$ of overnight cultures $\left(\mathrm{OD}_{600}\right.$ of 0.5$)$ into $4 \mathrm{~mL}$ of serial 2fold tigecycline dilutions in freshly prepared Mueller-Hinton Broth supplemented with $50 \mu \mathrm{g} /$ $\mathrm{mL}$ kanamycin, followed by 18 hours of incubation at $37^{\circ} \mathrm{C}$ at $130 \mathrm{rpm}$. MIC measurement was also conducted for $A$. baumannii strain AYE with or without tigecycline pre-exposure. Experiments were conducted with $n \geq 4$. The drug agar plate assays were conducted as described in 2.2.5.1, in which the serial dilution cultures were spotted on LB agar plate supplemented with $50 \mu \mathrm{g} / \mathrm{mL}$ kanamycin and tigecycline of different concentrations.

\subsubsection{RNA extraction and RT-qPCR}

Overnight cultures of $A$. baumannii strain AYE were inoculated into fresh $50 \mathrm{~mL}$ LB medium to an $\mathrm{OD}_{600}$ of 0.05 and grown at $37^{\circ} \mathrm{C}$ with $130 \mathrm{rpm}$. Tigecycline $(1 \mu \mathrm{g} / \mathrm{mL})$ was added to the cells when the cell culture reached the early mid-log phase $\left(O D_{600}\right.$ of approximately 0.4$)$. Cells of $500 \mu \mathrm{L}$ were harvested after 90 minutes. For untreated samples (control), cells at the same growth phase as the tigecycline-treated samples were collected. The total RNA extraction was conducted as described in 2.2.2.1 with the exception that after DNA digestion, the RNA samples were purified using RNA Clean \& Concentrator-5 (Zymo Research).

The quality and quantity of the RNA samples were determined using Nanodrop. One-step RT-qPCR was conducted on a Rotor-Gene Q (Qiagen) using Luna Universal One-Step RTqPCR kit (NEB). The RNA samples were adjusted to $10 \mu \mathrm{g} / \mathrm{mL}$ and $1 \mu \mathrm{L}$ of RNA samples was added to the reaction mixture mentioned in Table 2.19. The RT-qPCR was carried out according to the programme described in Table 2.20. The expression of adeB, adeG, adeJ and tet $A$ was quantified using $r p o B$ as reference gene. The primers used were listed in Table 2.3. 
Table 2.19 Reaction mixture for RT-qPCR using Luna Universal One-Step RT-qPCR kit.

\begin{tabular}{|l|c|}
\hline Chemicals & Volume $(\boldsymbol{\mu L})$ \\
\hline Luna Universal One-Step Reaction Mix $(2 \mathrm{x})$ & 5.0 \\
\hline Luna WarmStart RT Enzyme Mix $(20 \mathrm{x})$ & 0.5 \\
\hline Forward primer $(10 \mu \mathrm{M})$ & 0.5 \\
\hline Reverse primer $(10 \mu \mathrm{M})$ & 0.5 \\
\hline Template RNA $(10 \mu \mathrm{g} / \mathrm{mL})$ & 1.0 \\
\hline Nuclease-free water & 2.5 \\
\hline Total volume & 10.0 \\
\hline
\end{tabular}

Table 2.20 Programme for RT-qPCR using Luna Universal One-Step RT-qPCR kit.

\begin{tabular}{|l|l|l|c|}
\hline Steps & Temperature & Time & Cycles \\
\hline Reverse transcription & $55^{\circ} \mathrm{C}$ & 10 minutes & 1 \\
\hline $\begin{array}{l}\text { PCR initial heat } \\
\text { activation }\end{array}$ & $95^{\circ} \mathrm{C}$ & 1 minute & 1 \\
\hline Denaturation & $95^{\circ} \mathrm{C}$ & 10 seconds & 40 \\
\hline $\begin{array}{l}\text { Combined } \\
\text { annealing/extension }\end{array}$ & $60^{\circ} \mathrm{C}$ & 30 seconds & 1 \\
\hline melt curve analysis & $65^{\circ} \mathrm{C}-95^{\circ} \mathrm{C}$ & 5 seconds for each ${ }^{\circ} \mathrm{C}$ & \\
\hline
\end{tabular}




\section{CHAPTER 3 : RESULTS}

\subsection{Construction of $A$. baumannii ATCC 19606 efflux pump knockout strains}

To study the importance of specific efflux pumps in A. baumannii ATCC 19606, the efflux pump genes were deleted from the chromosomal DNA of the strain. The construction of knockout strain is based on the homologous recombination between the regions flanking the gene of interest, which are introduced into the bacteria by pBIISK_sacB/kanR_updown plasmid, and integrated onto the bacterial chromosomal DNA (Figure 3.1a).

a.
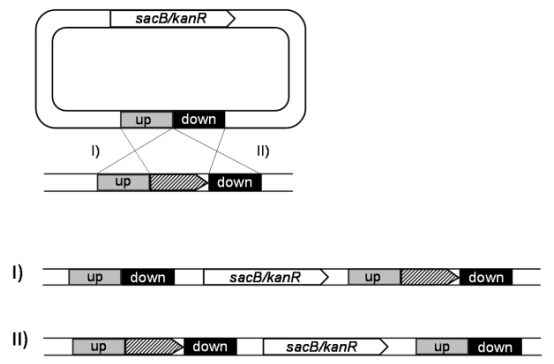

c.

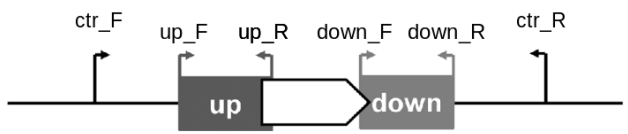

b.

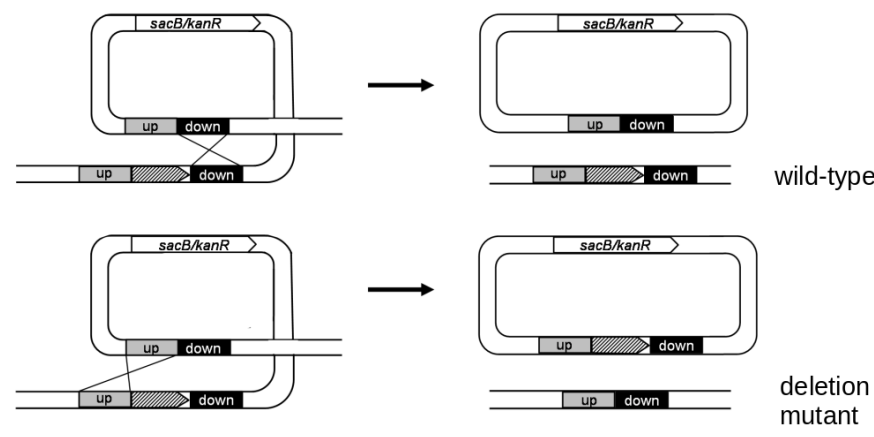

Figure 3.1 Gene knockout via homologous recombination. (a) Plasmid integration via the upstream or downstream homologous sequence. (b) Sucrose counter selection in the absence of antibiotic selective pressure promotes the second homologous recombination event for the selection of the plasmid backbone containing sacB/kanR, which results in the desired gene deletion or reversion to wild-type. (c) The position of primers used for the first and the second round of colony-PCR.

The plasmid pBIISK_sacB/kanR_updown harbours the upstream and downstream regions of the gene of interest, which are approximately 1500 bp each. PCR amplification of the upstream and downstream regions was carried out using genomic DNA of $A$. baumannii ATCC 19606 (Figure 3.2a). Next, the upstream fragment was inserted into pBIISK_sacB/kanR, either through restriction digestion or Gibson Assembly method. The 
resulting plasmid was verified by agarose gel electrophoresis and DNA sequencing, and served as template to insert the downstream fragment. The final plasmid construct containing both upstream and downstream DNA regions was again verified by gel electrophoresis (Figure 3.2b) and DNA sequencing (Eurofin).

The constructed plasmids (pBIISK_sacB/kanR_updown) were transformed into $A$. baumannii ATCC 19606 cells via electroporation and clones with kanamycin resistance were selected. Colonies were subjected to a first round of colony-PCR, in order to check whether the homologous recombination took place at the correct position. Two pairs of primer were used for the PCR [(ctr_F and down_R) and (up_F and ctr_R)] (Figure 3.1c), in which a bigger (upstream + gene of interest + downstream) and a smaller size (only upstream and downstream regions, around $3000 \mathrm{bp}$ ) products were expected (Figure 3.3).

a.

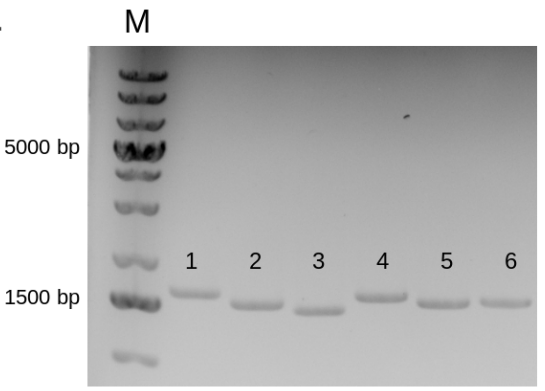

b.

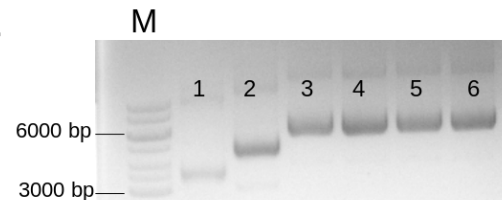

$\mathrm{M}$

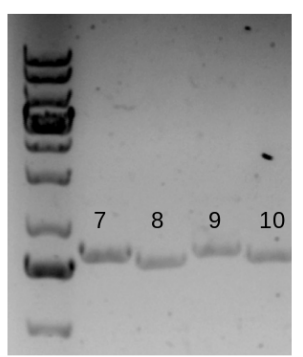

1 - abeS upstream

2 - abeS downstream

3 - adelJ upstream

4 - adelJ downstream

5 - craA upstream

6 - craA downstream

7 - ade $A B$ upstream

8 - ade $A B$ downstream

9 - adeFG upstream

10 - adeFG downstream

1 - empty vector (pBIISK_sacB/kanR)

2 - pBIISK_sacB/kanR_up

3 - pBIISK_sacB/kanR_updown (plasmid 1)

4 - pBIISK_sacB/kanR_updown (plasmid 2)

5 - pBIISK sacB/kanR_updown (plasmid 3)

6 - pBIISK_sacB/kanR_updown (plasmid 4)

Figure 3.2 Cloning of pBIISK_sacB/kanR_updown. (a) Agarose gel electrophoresis analysis of the PCR amplifications of the upstream and downstream DNA regions (approximately $1500 \mathrm{bp}$ ) of the genes of interest. (b) Plasmids were subjected to gel electrophoresis to monitor the insertion of the upstream and downstream DNA regions into the vector. $M=$ marker (GeneRuler DNA ladder $1 \mathrm{~kb}$ ). 
a.

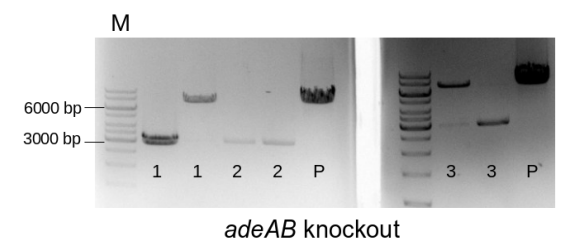

d.

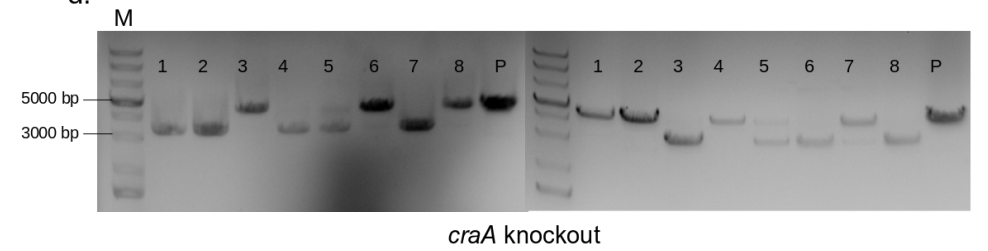

b.

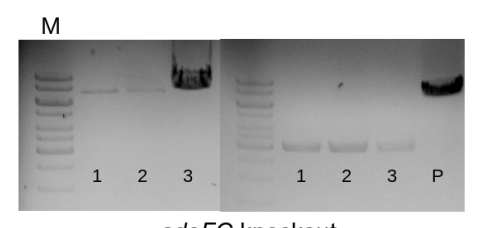

adeFG knockout

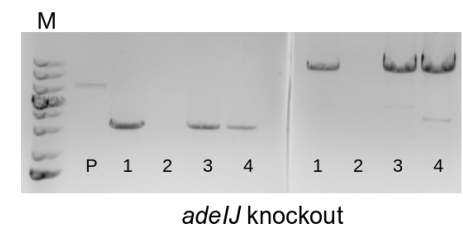

e.

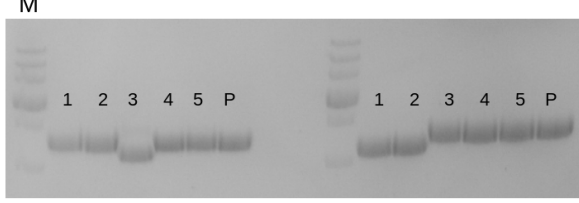

abes knockout

Figure 3.3 First round of colony-PCR to monitor the integration of plasmid. Correct integrants showed a smaller PCR product ( $\sim 3000 \mathrm{bp}$ ) for one of the primer pairs and a larger PCR product (same size as positive control P) for another primer pair. $\mathrm{P}=$ positive control using the genomic DNA of wild-type A. baumannii ATCC 19606 as template, $M=$ marker (GeneRuler DNA ladder $1 \mathrm{~kb}$ ). (a) adeAB knockout: colony 1 and 3 are correct integrants. (b) adeFG knockout: colony 1, 2 and 3 are correct integrants. (c) adelJ knockout: colony 1, 3 and 4 are correct integrants. (d) craA knockout: colony 1, 2, 3, 4, 6, 7, 8 are correct integrants. (e) abeS knockout: colony 1, 2, 3 are correct integrants.

Colonies that exhibited correct bands were then subjected to two rounds of counter selection using sucrose. The sacB gene originated from $B$. subtilis encodes levan sucrase that converts sucrose to levans, which is harmful to $A$. baumannii. Thus, a second homologous recombination event resulted in elimination of the $s a c B / k a n R$ cassette, which might result the desired deletion mutant or wild-type revertants (Figure 3.1b). To determine whether the counter selection was successful, $A$. baumannii colonies were picked and replica plated to LB agar in the presence and absence of kanamycin. Only colonies that could not grow on the LB agar in the presence of kanamycin were subjected to the second round of colonyPCR using the primer pair ctr_F and ctr_R (Figure 3.1c). The willd-type revertants have a larger size of PCR products (similar as the positive control), while successfully knocked out colonies have a PCR product size of around 3000 bp (Figure 3.4). Expected gene deletions were confirmed by DNA sequencing (Eurofin). 


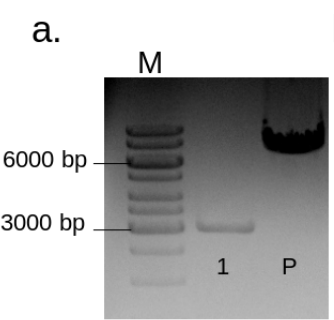

ade $A B$ knockout

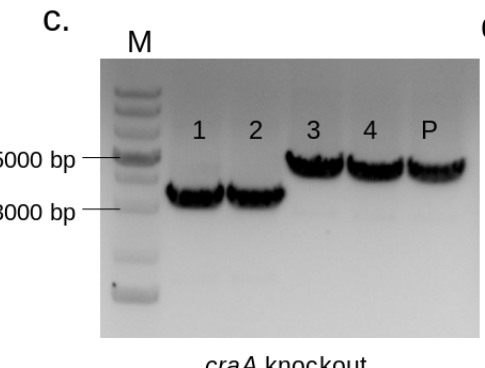

b.

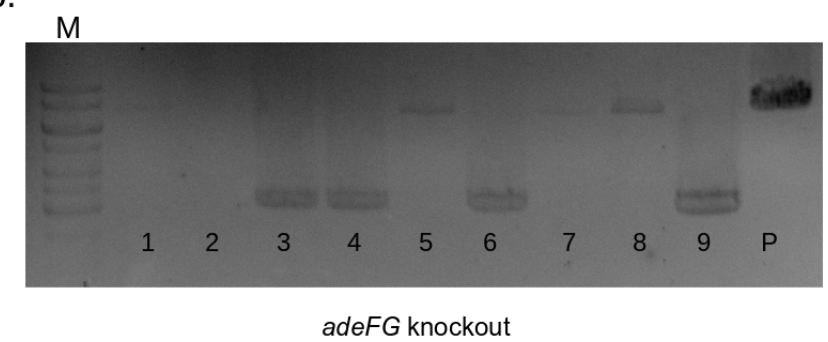

d.

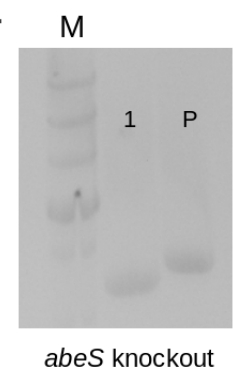

e.

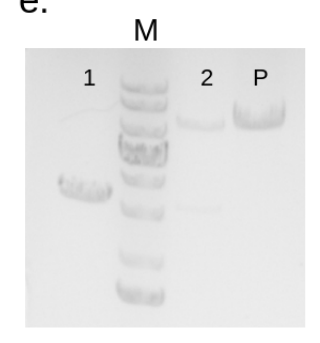

adelJ knockout

Figure 3.4 Second round of colony-PCR using the primer pair ctr_F and ctr_R. Successful deletion mutants showed PCR products with a size of $\sim 3000 \mathrm{bp}$, while wild-type revertants have the same size as the positive control $(\mathrm{P})$. The different colonies are represented by numbers, $\mathrm{P}=$ positive control using the genomic DNA of wild-type $A$. baumannii ATCC 19606 as template, $\mathrm{M}=$ marker (GeneRuler DNA ladder $1 \mathrm{~kb}$ ). (a) adeAB knockout: colony 1 is a deletion mutant. (b) adeFG knockout: colony 3, 4, 6 and 9 are deletion mutants. (c) craA knockout: colony 1 and 2 are deletion mutants. (d) abeS knockout: colony 1 is a deletion mutant. (e) adelJ knockout: colony 1 is a deletion mutant. Knockout strain of abeS was not used in any experiment in this thesis.

\subsection{Differential gene expression analysis of knockout strains}

The upregulation of efflux pump genes and low membrane permeability are the major antibiotic resistance mechanisms in $A$. baumannii (explained in Chapter 1). The expression of RND genes, craA and ompA for the wild-type strain, RND- or CraA efflux pumps knockout strains were examined. In addition, the differential gene expression of efflux pumps and outer membrane protein of the wild-type and knockout strains under chloramphenicol stress was also investigated.

\subsubsection{Sample preparation for RNA extraction}

Prior to the growth of cells for RNA extraction, MIC measurement was conducted to determine the subminimal inhibitory concentration of chloramphenicol to be utilized for cell growth (Table 3.1). The concentration of chloramphenicol added should not be too high to 
inhibit the cell growth, or retard the growth too extensively. A. baumannii ATCC 19606 WT, $\triangle a d e A B, \triangle a d e l J$ or $\triangle c r a A$, were grown in LB medium supplemented with subinhibitory concentrations of chloramphenicol. When $\mathrm{OD}_{600}$ of the cell cultures reached 0.5 to 0.7 , samples were collected for RNA extraction. For untreated samples (control), cells were grown to the same growth phase in LB medium without chloramphenicol.

Table 3.1 MIC and sub-MIC values for chloramphenicol utilized for growth of the indicated strains of $A$. baumannii.

\begin{tabular}{|c|c|c|}
\hline A. baumannii ATCC 19606 & $\begin{array}{c}\text { MIC of chloramphenicol } \\
(\boldsymbol{\mu} \mathbf{g} / \mathbf{m L})\end{array}$ & $\begin{array}{c}\text { sub-MIC of chloramphenicol } \\
(\boldsymbol{\mu g} / \mathbf{m L})\end{array}$ \\
\hline WT & 64 & 8 \\
\hline$\Delta c r a A$ & 2 & 0.5 \\
\hline$\Delta$ adeAB & 64 & 8 \\
\hline$\Delta$ adelJ & 16 & 4 \\
\hline
\end{tabular}

RNA samples were checked with Nanodrop to determine the RNA quality and quantity. Good quality RNA samples exhibit a ratio of absorbance at $260 \mathrm{~nm}$ to $280 \mathrm{~nm}$ around 2.0 and even higher ratio for the absorbance at $260 \mathrm{~nm}$ to $230 \mathrm{~nm}$. The RNA samples that showed $260 / 280$ ratio between 2.0 to 2.2 and $260 / 230$ ratio above 2.0 were used for the subsequent RT-qPCR experiment.

\subsubsection{Quantitative reverse transcription PCR (RT-qPCR)}

\subsubsection{Determination of PCR efficiency and melt curve analysis}

To determine whether the RNA samples and primer design for qPCR assay are optimal, a standard curve analysis was conducted using duplicate ten-fold serial dilutions of RNA sample as template (Figure 3.5a). A standard curve was constructed by plotting the log of the template concentrations against the $C_{T}$ (cycle threshold) values (Figure 3.5b). The equation of the linear regression line, along with Pearson correlation coefficient $(R)$ or the coefficient of determination $\left(R^{2}\right)$, which represents how well the experimental data fit the regression line, can be used to evaluate whether the GPCR assay is optimal. Under the condition of a 'perfect' PCR, the DNA doubles with each amplification cycle. Thus, for a tenfold serial dilution of template, $2^{n}=10, n=3.32$, where $n$ is the difference between the $C_{T}$ 
values of the curve. Evenly spaced amplification curves will produce a linear standard curve with a slope close to -3.32 and reaction efficiency close to 1.0 or $100 \%$. $R>0.990$ or $R^{2}>0.980$ is also expected for a PCR run with high reaction efficiency.

Low reaction efficiency $(<90 \%)$ is often caused by contamination of impurities in the RNA samples, poorly designed primers, or a less than optimal annealing temperature. A reaction efficiency which is too high $(>110 \%)$ can be due to the formation of primer-dimers or nonspecific amplicons. The presence of impurities might also lead to high reaction efficiency. In this work, the PCR runs with the efficiency more than $95 \%$ and less than $105 \%$ were considered valid. RNA extractions were repeated if the PCR efficiency for the sample was out of the range.

a.

\begin{tabular}{|c|c|c|c|}
\hline Sample & RNA Concentration $(\mathrm{ng} / \mathrm{\mu L})$ & $\mathrm{C}_{\mathrm{T}}$ for replicate 1 & $\mathrm{C}_{\mathrm{T}}$ for replicate 2 \\
\hline \multirow{3}{*}{$\mathrm{WT}$} & 20.0 & 18.85 & 17.97 \\
\cline { 2 - 4 } & 2.0 & 19.66 & 20.05 \\
\cline { 2 - 4 } & 0.2 & 22.94 & 23.02 \\
\cline { 2 - 4 } & 0.02 & 26.00 & 26.71 \\
\cline { 2 - 4 } & 0.002 & 30.35 & 30.27 \\
\cline { 2 - 4 } & 0.0002 & 33.69 & 34.54 \\
\hline
\end{tabular}

b.

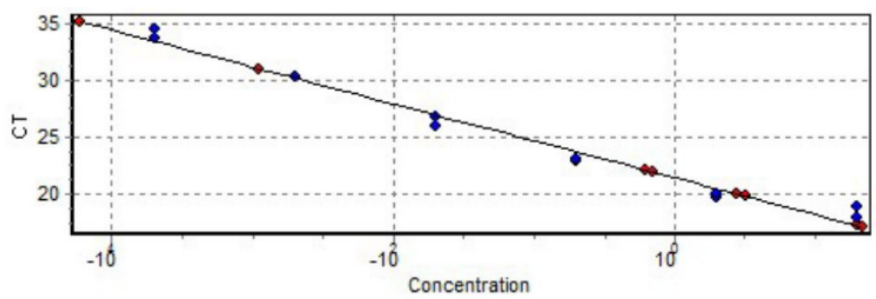

\begin{tabular}{|l|l|}
\hline$M$ (slope) & -3.23595 \\
\hline$R$ value & 0.99009 \\
\hline$R^{2}$ value & 0.98028 \\
\hline $\begin{array}{l}\text { Reaction } \\
\text { efficiency }\end{array}$ & $10^{(-1 / M)}-1=1.03717$ \\
\hline
\end{tabular}

Figure 3.5 Standard curve analysis to determine PCR efficiency. (a) An example of standard curve analysis using the sample WT and the primer rpob (b) Standard curve plotted from the data of (a).

As SYBR Green I binds to all double-stranded DNA, it is important to check the specificity of the PCR by analyzing the end products. melt curve analysis was conducted after every PCR run by gradually increasing the temperature gradually $\left(1^{\circ} \mathrm{C}\right.$ every 5 seconds). As the DNA denatures, the fluorescence decreases. The specific melting temperature (Tm) for each amplicon can distinguish it from other non-specific products or primer dimers (Figure 3.6). A 
single peak indicates a single PCR product, confirming that the primers used for each gene amplification are specific (Figure 3.6).

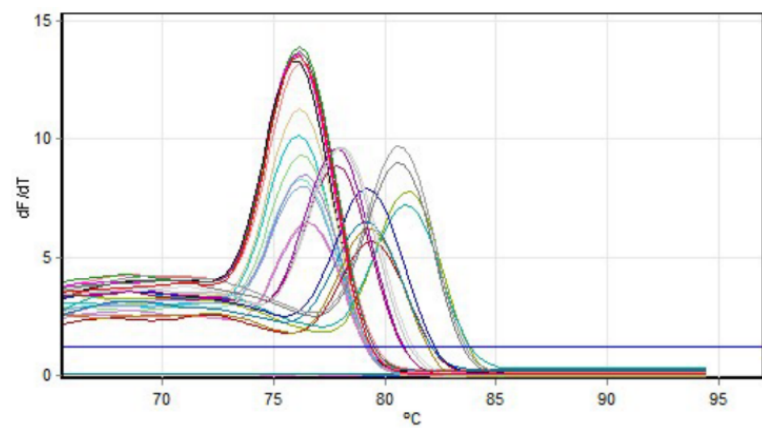

\begin{tabular}{|l|l|}
\hline Gene & $\operatorname{Tm}\left({ }^{\circ} \mathbf{C}\right)$ \\
\hline rpob & 76.2 \\
\hline adeB & 79.5 \\
\hline adeG & 79.2 \\
\hline adeJ & 81.0 \\
\hline craA & 77.8 \\
\hline ompA & 80.5 \\
\hline
\end{tabular}

Figure 3.6 An example of melt curve analysis conducted to determine the primer specificity. The melting temperature $(\mathrm{Tm})$ for each gene is indicated in the table.

\subsubsection{Differential gene expression analysis}

The expression of five genes (adeB, adeG, adeJ, cra $A$ and ompA) of four $A$. baumannii ATCC 19606 strains (WT, $\triangle a d e A B, \triangle a d e l J$ and $\triangle c r a A$ ), with or without the presence of chloramphenicol were studied. $\Delta \mathrm{C}_{\mathrm{T}}$ was obtained from $\mathrm{C}_{\mathrm{T}(\text { r } p o b)}-\mathrm{C}_{\mathrm{T} \text { (gene of interest) }}$ of at least three biological replicates. Higher $\Delta \mathrm{C}_{\mathrm{T}}$ values indicate higher amount of mRNA transcripts. The difference between the $\Delta \mathrm{C}_{\mathrm{T}}$ of the treated and the control group yields the $\Delta \Delta \mathrm{C}_{\mathrm{T}}$ value. Positive $\Delta \Delta \mathrm{C}_{\mathrm{T}}$ value indicates upregulation, while negative value indicates downregulation of the target gene due to the treatment (knockout of certain gene or the presence of chloramphenicol).

Knockout of adelJ, ade $A B$ or craA did not cause significant changes in the gene expression for adeB, adeJ, adeG, craA and ompA in comparison to the wild-type $A$. baumannii ATCC 19606 , as indicated by the $\Delta \Delta \mathrm{C}_{\mathrm{T}}$ values which are close to zero (Figure 3.7). 
a.

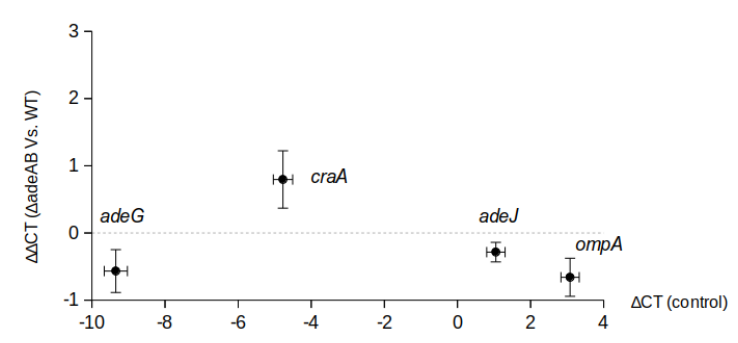

C.

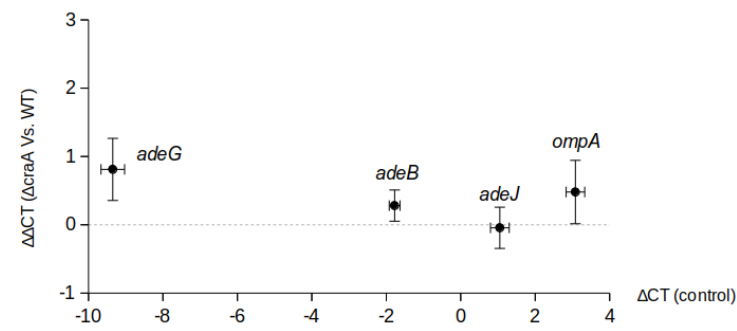

b.

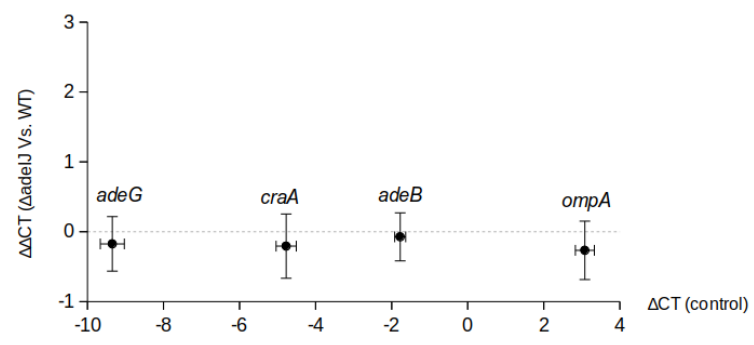

Figure 3.7 Differential gene analysis of RND efflux pump genes, craA and ompA of $A$. baumannii ATCC 19606 between the knockout and the wild-type strain. Points and error bars represent means and standard errors, respectively, of $n \geq 3$ independent experiments. The gene knockout of $A$. baumannii ATCC 19606 did not cause significant changes in the gene expression of ade $B$, adeJ, adeG, cra $A$ and ompA. (a) adeAB-knockout strain versus WT (b) adelJ-knockout strain versus WT (c) craA-knockout strain versus WT.

When chloramphenicol was added to the growth medium at a sub-MIC concentration, overexpression of craA was observed for the WT and knockout strains (except in $A$. baumannii $\triangle c r a A$ ) in comparison to the WT of control condition (Figure 3.8). This indicates that the MFS member CraA is the major chloramphenicol resistant determinant in $A$. baumannii ATCC 19606. In contrast to the drastically overexpressed $c r a A$, the expression level of adeB, adeG and adeJ were maintained under antibiotic stress (Figure 3.8). The high amount of transcripts (high $\Delta \mathrm{c}$ T value) indicates that adeJ is constitutively expressed at high level. The MIC of chloramphenicol in A. baumannii ATCC $19606 \Delta$ adeAB which shows no difference with the wild-type strain (Table 3.1) indicates that the AdeABC system does not play an important role in chloramphenicol transport. Similar prediction was applied to AdeFGH, due to the low amount adeG transcripts (Figure 3.8). Apart from the efflux pump genes, the expression of ompA was checked. No obvious up- or downregulation was observed between the WT strain and the knockout strains under control or treated conditions (Figure 3.8). 
a.

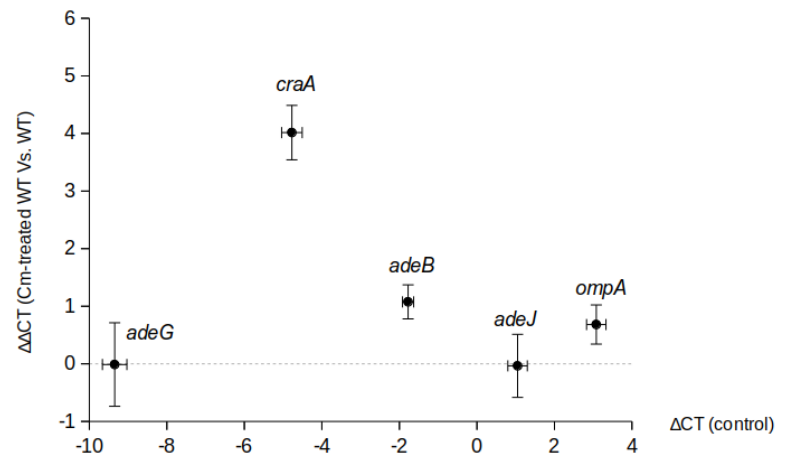

C.

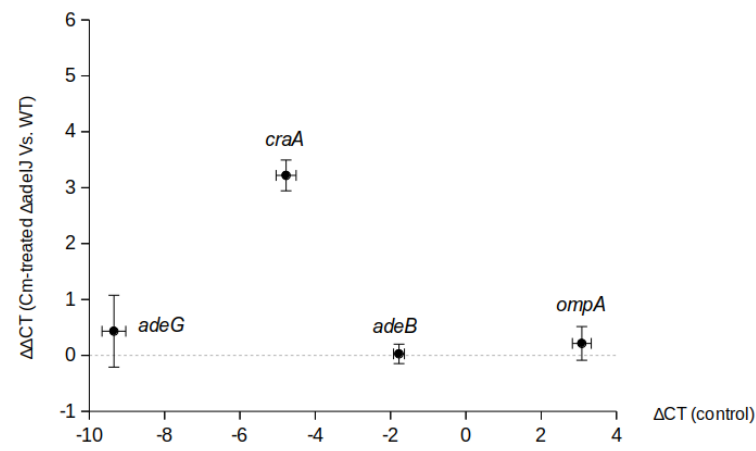

b.

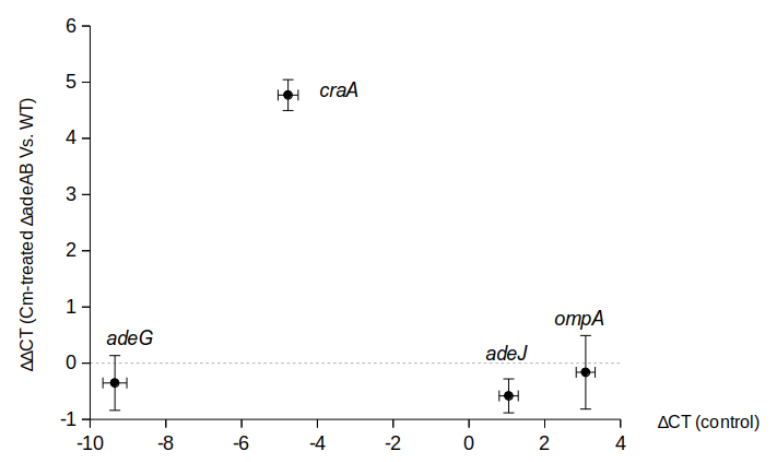

d.

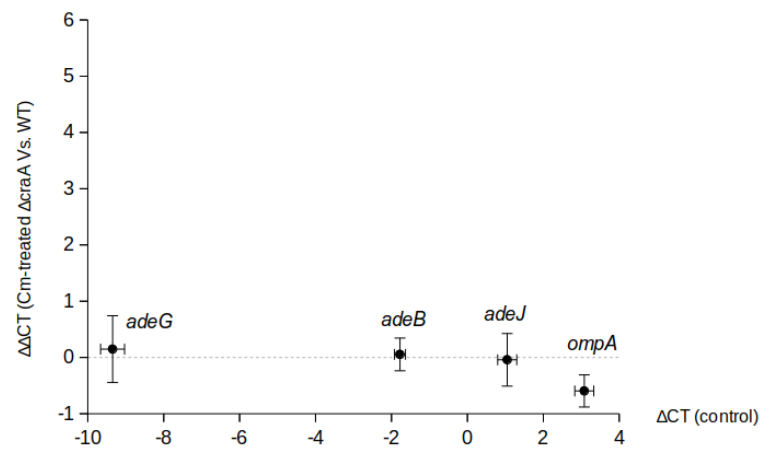

Figure 3.8 Differential gene analysis of RND efflux pump genes, craA and ompA of $A$. baumannii ATCC 19606 between the treatment group and the control (wild-type strain without chloramphenicol treatment). Points and error bars represent means and standard errors, respectively, of $n \geq 3$ independent experiments. The presence of sub-MIC chloramphenicol has caused significant overexpression of $c r a A$, while the expression of ade $B$, adeJ, ade $G$ and ompA remained stable. (a) chloramphenicol-treated $\triangle$ ade $A B$ versus WT (b) chloramphenicol-treated $\triangle$ adelJ versus WT (c) chloramphenicol-treated $\triangle$ adeAB versus WT (d) chloramphenicol-treated $\triangle$ craA versus WT.

In conclusion, the results of differential gene analysis suggest that the deletion of single RND-type (adeAB or adelJ) or MFS-type (craA) efflux pump gene did not influence the gene expression of other efflux pumps or the major outer membrane protein ompA gene. The presence of chloramphenicol has induced the overexpression of $c r a A$ in wild-type and knockout strains, indicating that $c r a A$ is the major chloramphenicol resistant determinant in A. baumannii ATCC 19606. 


\subsection{Characterization of CraA}

\subsubsection{Putative chloramphenicol transporters in A. baumannii strain AYE}

The whole-genome sequencing of $A$. baumannii strain AYE, a multidrug-resistant and epidemic strain in France (Poirel et al., 2003 ; Fournier et al., 2006), revealed five genes which are putative chloramphenicol transporters. In order to characterize these transporters, the genes were cloned and heterologously expressed in E. coli. Deletion strain of $m d f A$ and emrE was utilized, as these multidrug efflux pumps might have overlapping substrate specificity with the putative chloramphenicol transporters in A. baumannii strain AYE.

The genes amplified via PCR using the genomic DNA of $A$. baumannii strain AYE as template were inserted into pTTQ18 vector backbone. Subsequently, these putative multidrug chloramphenicol transporters were overexpressed in $E$. coli BW25113 $\triangle e m r E \Delta m d f A$ and phenotypic screening was conducted to determine the resistance profile of these $E$. coli strains harbouring the $A$. baumannii chloramphenicol transporter genes.

The result shows that $c r a A$ - and cmIA5-expressing cells conferred resistance against chloramphenicol compared to the negative control, whereas cells expressing gene ABAYE0913 and ABAYE0685 were susceptible to chloramphenicol (Figure 3.9). However, ABAYE0913-expressing cells exhibited resistance against florfenicol and benzalkonium. Cells expressing cmIA5 showed similar degree of resistance against thiamphenicol in comparison to craA-expressing cells but not to florfenicol. Cells overexpressing $\mathrm{cm} / \mathrm{A}$ did not grow well, probably due to the toxicity caused by protein production after induction with IPTG, as implied by the results on the control plate (Figure 3.9). Interestingly, cells expressing $c r a A$ exhibited resistance not only against chloramphenicol, but also against thiamphenicol, florfenicol, dequalinium, and benzalkonium. This transporter was further analysed to determine its substrate specificity. 


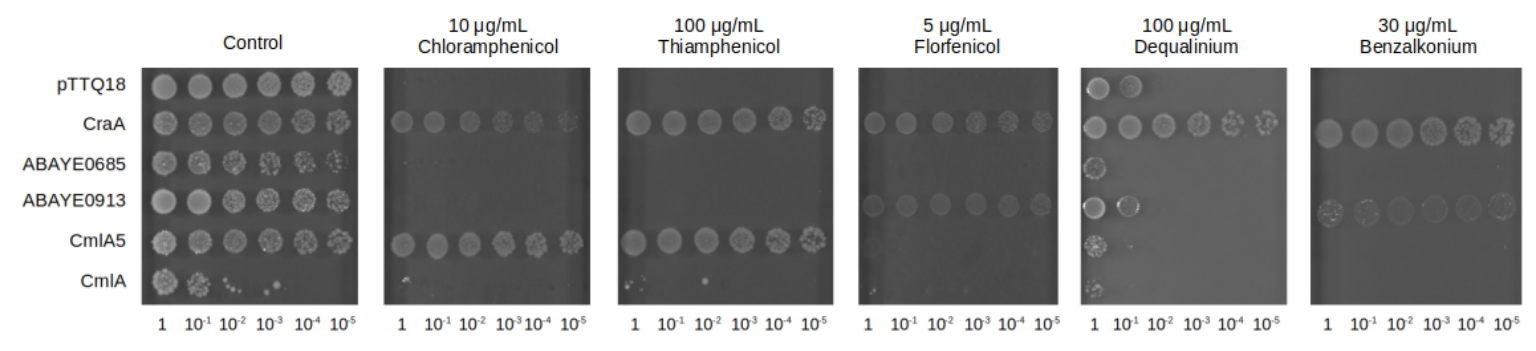

Figure 3.9 Drug susceptibility assays of E. coli BW25113 $\Delta$ emrEAmdfA harbouring putative chloramphenicol transporter genes from $A$. baumannii strain AYE. Serial diluted cell cultures (dilution given below the plate figures) harbouring empty vector (pTTQ18) or transporter genes were spotted on LB agar plates supplemented with 0.2 $\mathrm{mM}$ IPTG, $100 \mu \mathrm{g} / \mathrm{mL}$ ampicillin and the indicated drugs (substrates and concentrations given above the plate figures). Cells harbouring empty vector PTTQ18 are included as a negative control. Experiments were conducted at least three times and the results shown here are representative.

Drug susceptibility assays with an expanded substrate spectrum was conducted on cells overexpressing $c r a A$ and it was confirmed that this transporter conferred resistance against phenicols (chloramphenicol, florfenicol and thiamphenicol), monovalent cationic compounds (ethidium, benzalkonium, TPP ${ }^{+}$), fluoroquinolones (norfloxacin and ciprofloxacin), divalent cationic compounds with long linker (chlorhexidine and dequalinium), and a chemotherapeutic agent (mitomycin C) (Figure 3.10).

a.

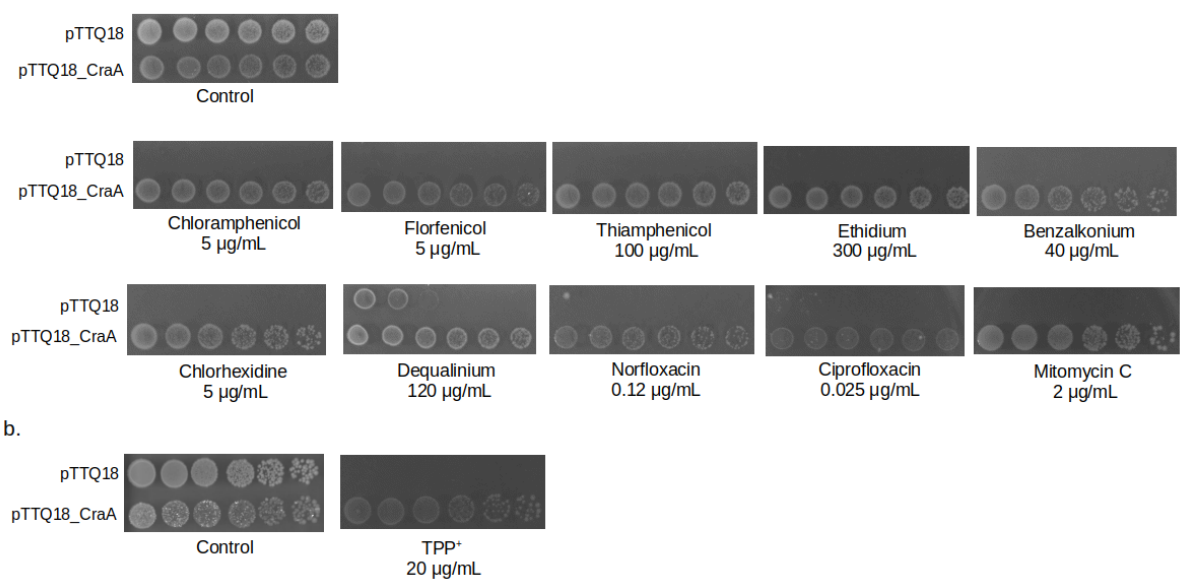

Figure 3.10 Drug susceptibility assays of $E$. coli deletion mutants harbouring $\operatorname{cra} A$ of $A$. baumannii strain AYE. The assays were conducted using (a) E. coli BW25113 $\Delta$ emrEAmdfA or (b) E. coli BW25113 $\triangle$ emrE $\Delta m d f A \Delta a c r A B$ complemented with pTTQ18_craA on LB agar plates supplemented with 0.2 mM IPTG, 100 $\mu \mathrm{g} / \mathrm{mL}$ ampicillin and the indicated drugs (drugs and concentrations given below the plate figures). Cells harbouring empty vector pTTQ18 was included as a negative control. Experiments were conducted at least three times and the results shown here are representative. 


\subsubsection{Drug susceptibility assays of CraA variants in E. coli}

Sequence alignment (Figure 3.11) and inspection of a MdfA-based (PDB: 6EUQ) homology model of CraA indicate that there are two conserved membrane-embedded proton-titratable residues, E26 and D34, in MdfA which are corresponding to E38 and D46 in CraA. CraA contains an additional negatively charged residue, E338, in the putative binding pocket (Figure 3.12).

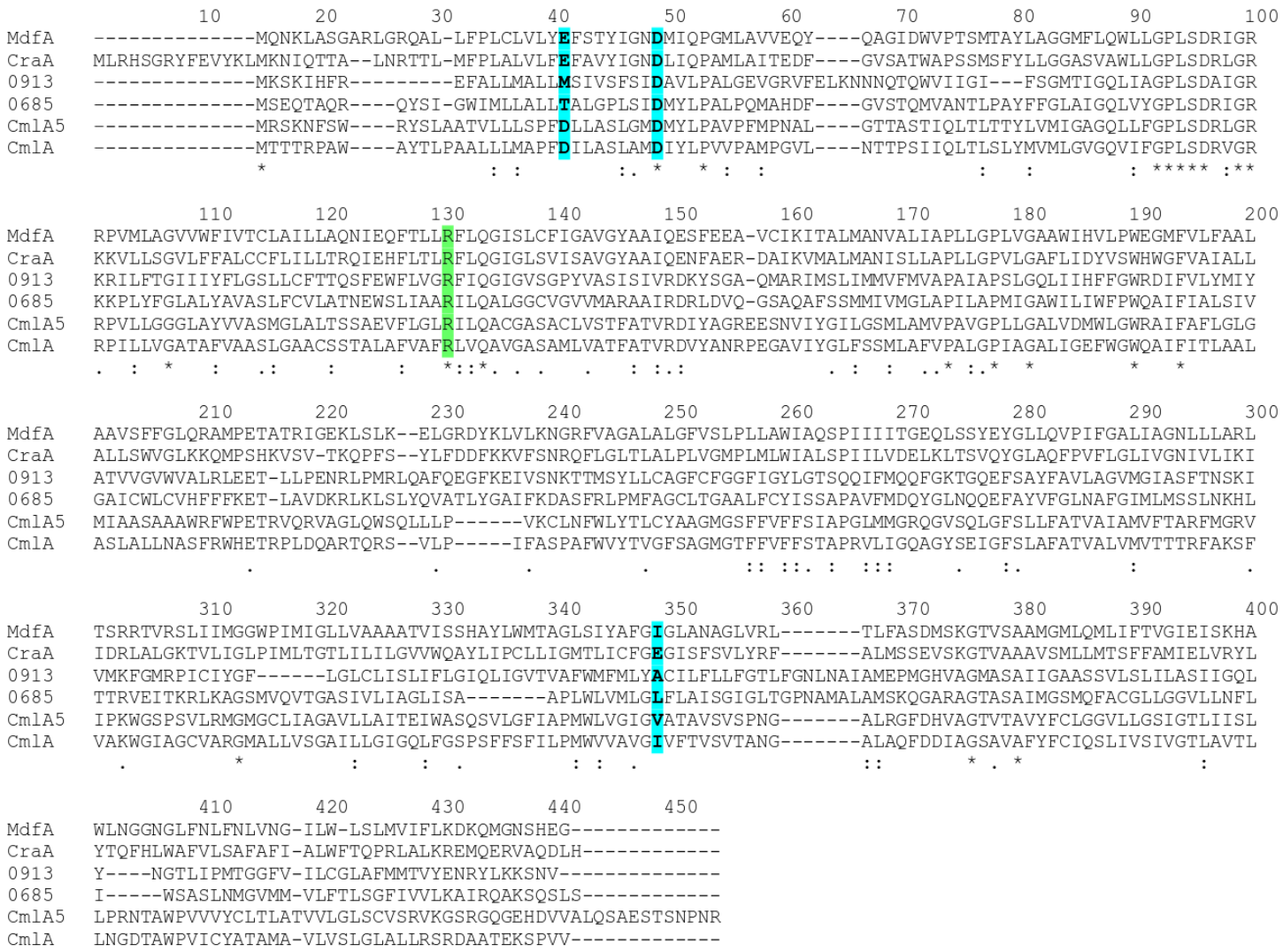

Figure 3.11 Protein sequence alignment of MdfA of $E$. coli and five putative chloramphenicol transporters of $A$. baumannii strain AYE, which are CraA (GenBank accession number: CAJ77876), CmIA (GenBank accession number: CAM88409), CmIA5 (GenBank accession number: CAM88395), 0685 (GenBank accession number: CAM85649) and 0913 (GenBank accession number: CAM85858). The residues highlighted in blue indicates the membrane-embedded negatively charged residues of CraA. Residues highlighted in green are the conserved argenine residue (R112) in MdfA orthologs. 


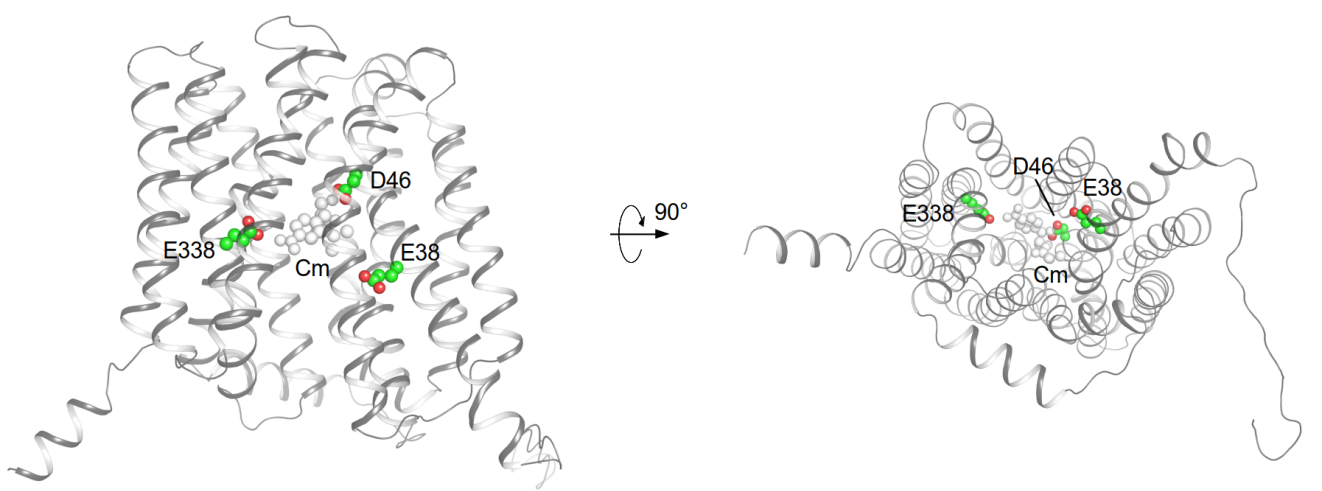

Figure 3.12 Homology model of CraA. Model was obtained based on the MdfA-Q131A_L339A variant (PDB: 6EUQ) (Zomot et al., 2018). Three membrane-embedded carboxylate residues and chloramphenicol (Cm) are depicted as spheres.

Apart from these three residues, several residues located in the binding pocket are estimated to be important for the binding and transport of substrates. To evaluate the importance of these residues for drug and/or $\mathrm{H}^{+}$transport, alanine-substitution variants of CraA were created. The drug susceptibility profile of E. coli BW25113 $\Delta$ emrE $\Delta m d f A$ harbouring CraA variants is summarized in Table 4.2. The Western blot analysis of the whole cell extracts confirmed that all CraA variants expressed equally well compared to wild-type CraA (Figure 3.17). Two bands were observed for all samples due to the presence of two populations of proteins, i.e. the folded and partly misfolded proteins.

The study on the three membrane-embedded titratable residues shows that E. coli BW25113 $\triangle$ emrEAmdfA expressing craA_E38A confer no resistance against all tested substrates, while craA_D46A-expressing cells conferred resistance to a lesser extent against florfenicol and benzalkonium and is active against norfloxacin (Figure 3.13a,b ; Table 3.2). Neutralizing E38 in the background of D46A completely abolished the resistance against all tested drugs. The variant E338A conferred slight resistance against phenicols, benzalkonium and ethidium (Figure 3.13a,b ; Table 3.2). The assay was in addition conducted using E. coli BW25113 $\triangle e m r E \Delta m d f A \triangle a c r A B$ as host. The concentrations of drugs adopted for similar effect in the cells without $A c r A B$ were much lower compared to the cells with AcrAB. This confirms that the RND tripartite system AcrAB-TolC plays an important role in the efflux-mediated antibiotic resistance and in this experiment possibly in synergy with CraA (Figure 3.13b). 

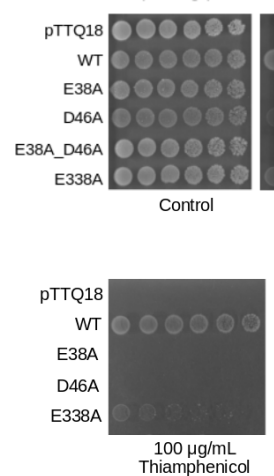
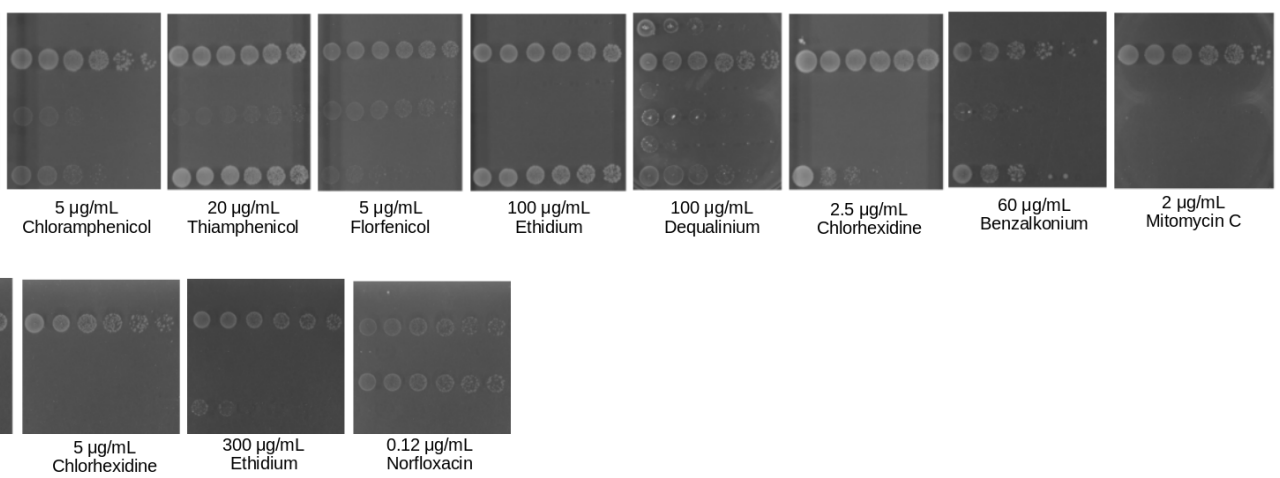

b.
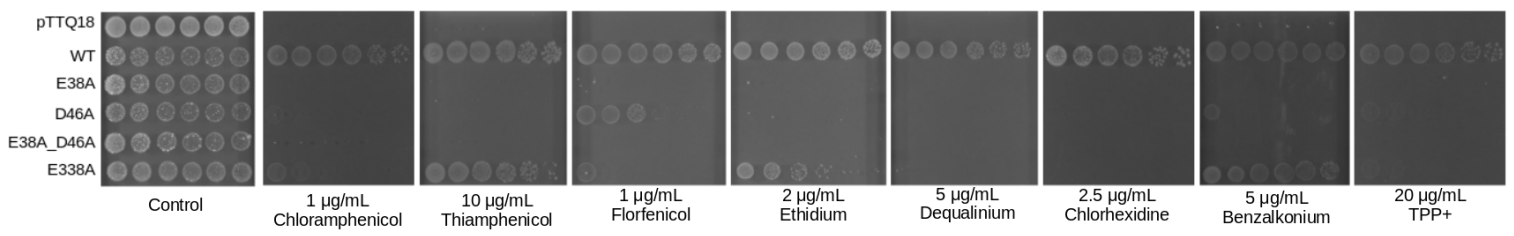

Figure 3.13 Drug susceptibility assays of $E$. coli deletion mutants harbouring craA (WT or variants of membraneembedded titratable residues). (a) E. coli BW25113 $\Delta$ emrE $\Delta m d f A$ or (b) E. coli BW25113 $\Delta$ emrE $\Delta m d f A \Delta a c r A B$ as host. Serial diluted cell cultures harbouring pTTQ18 (empty vector) or PTTQ18_craA (WT or variants) were spotted on LB agar plates supplemented with $0.2 \mathrm{mM}$ IPTG, $100 \mu \mathrm{g} / \mathrm{mL}$ ampicillin and the indicated drugs (drugs and concentration given below the plate figures). Cells harbouring empty vector PTTQ18 was included as a negative control. Experiments were conducted at least three times and the results shown here are representative.

Apart from alanine-substitution, E38 was substituted to histidine, glutamine and aspartate while D46 was substituted to histidine, asparagine and glutamate. CraA_E38Q was still able to confer resistance towards the electroneutral drugs chloramphenicol, thiamphenicol and florfenicol but not towards monocationic drug ethidium (Figure 3.14 ; Table 3.2), comparable to the phenotype conferred by the MdfA_E26Q mutant (Adler et al., 2004 ; Sigal et al., 2009).

Surprisingly, cells expressing craA_E38D were not active against most drugs except thiamphenicol and benzalkonium (Figure 3.14, Table 3.2). Both craA_D46A and craA_D46Nexpressing cells conferred resistance against norfloxacin, while the D46N mutant even showed tendency to be hyperactive against norfloxacin (Figure 3.14). In contrast, cell expressing craA_D46E conferred resistance against ethidium, benzalkonium, chlorhexidine, and dequalinium. The double mutants E38D_D46A and E38A_D46E did not show activity towards all tested drugs (Figure 3.14), a similar phenotype observed for double mutant E38A_D46A (Figure 3.13). 

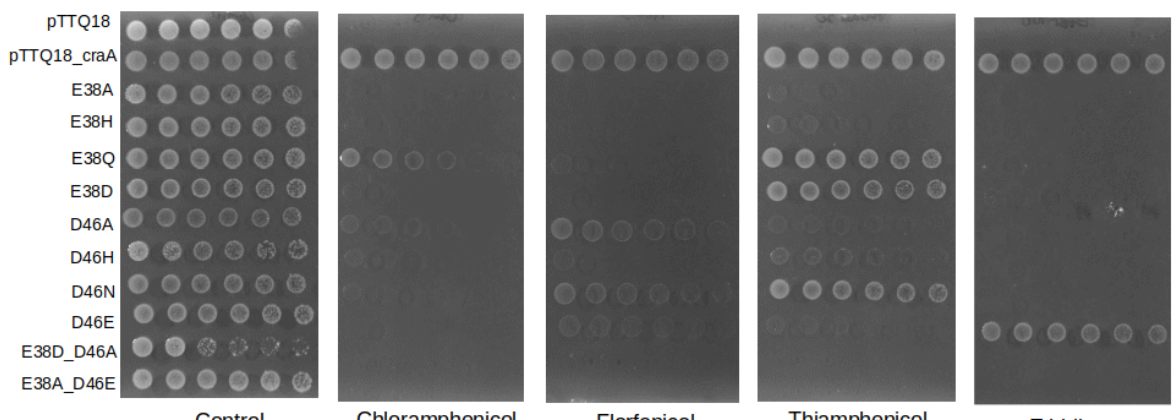

$5 \mu \mathrm{g} / \mathrm{mL}$
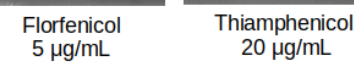

$20 \mu \mathrm{g} / \mathrm{mL}$

Ethidium
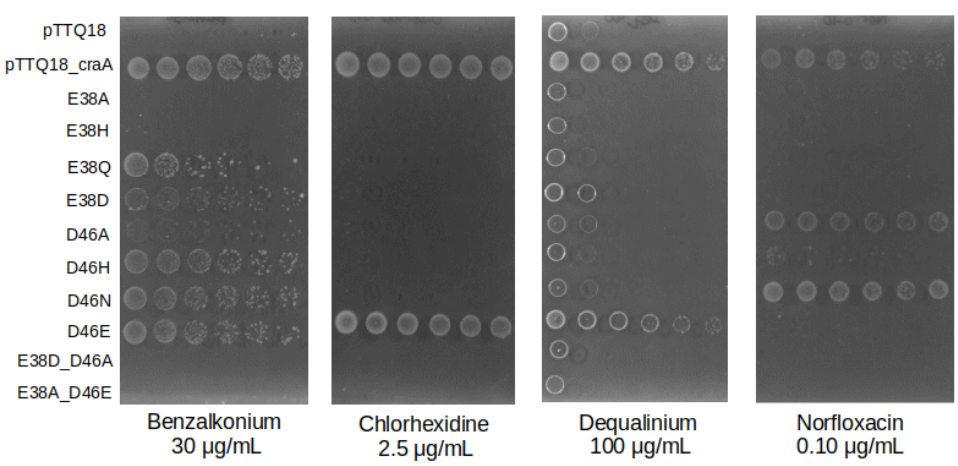

$100 \mu \mathrm{g} / \mathrm{mL}$

Figure 3.14 Drug susceptibility assays of E. coli BW25113 $\Delta$ emrEAmdfA harbouring pTTQ18_craA, E38 and D46 variants. Serial diluted cell cultures harbouring empty vector (pTTQ18) or pTTQ18_craA (WT or variants) were spotted on LB agar plates supplemented with $0.2 \mathrm{mM} \mathrm{IPTG}, 50 \mu \mathrm{g} / \mathrm{mL}$ carbenicillin and the indicated drugs (drugs and concentration given below the plate figures). Cells harbouring empty vector pTTQ18 are included as a negative control. Experiments were conducted at least three times and the results shown here are representative.

Substitution E38A renders CraA completely inactive, but also alanine-substitution of residues N45, Y73, and R124 (Figure 3.15, Table 3.2). The importance of tyrosine at position 73 was further studied by substitution to phenylalanine and leucine. The result shows that phenylalanine-substitution did not affect the activity of CraA whereas $\mathrm{Y} 73 \mathrm{~L}$ is completely inactive (Figure 3.15). The presence of a positive charge at position 112 was proven to be essential for the function of MdfA in E. coli and MdfA_R112H mutant is partially active (Sigal et al., 2005 ; Heng et al., 2015). However, in the case of CraA, replacement of arginine to histidine at this position $(\mathrm{R} 124 \mathrm{H})$ could not maintain the function CraA (Figure 3.15). 


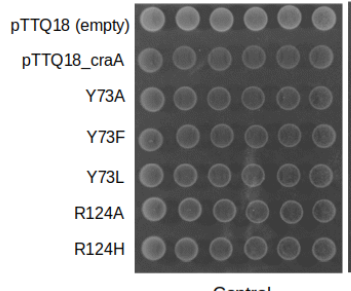

Control

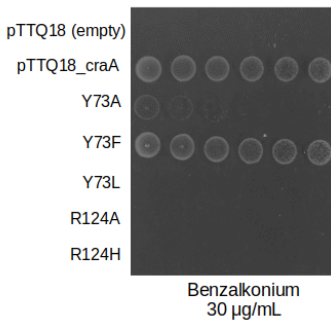

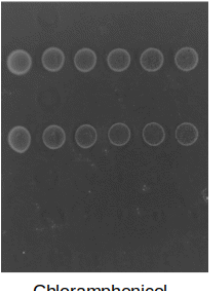

Chloramphenico $5 \mu \mathrm{g} / \mathrm{mL}$

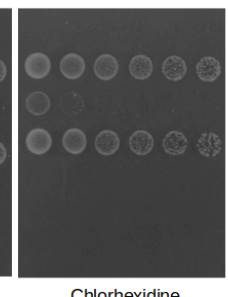

Chlorhexidine
$5 \mu \mathrm{g} / \mathrm{mL}$

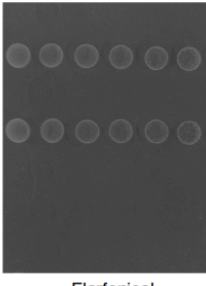

Florfenicol

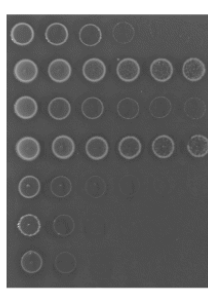

Dequalinium
$120 \mu \mathrm{g} / \mathrm{mL}$

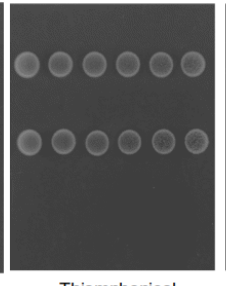

Thiamphenicol $20 \mu \mathrm{g} / \mathrm{mL}$

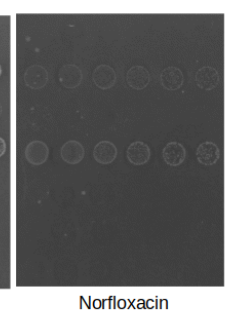

Norfloxacin
$0.15 \mu \mathrm{g} / \mathrm{mL}$

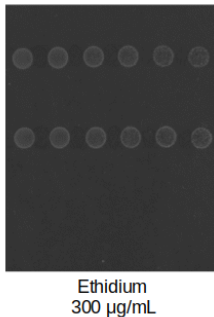

$300 \mu \mathrm{g} / \mathrm{mL}$

Figure 3.15 Drug susceptibility assays of E. coli BW25113 $\Delta$ emrEAmdfA harbouring pTTQ18_craA, Y73 and R124 variants. Serial diluted cell cultures harbouring empty vector (pTTQ18) or pTTQ18_craA (WT or variants) were spotted on LB agar plates supplemented with $0.2 \mathrm{mM}$ IPTG, $50 \mu \mathrm{g} / \mathrm{mL}$ carbenicillin and the indicated drugs (drugs and concentration given below the plate figures). Cells harbouring empty vector pTTQ18 are included as a negative control. Experiments were conducted at least three times and the results shown here are representative.

Apart from the above mentioned E38A and R124A, CraA variant N45A, P166A and N283A also conferred different extents of susceptibility towards all tested drugs (Figure 3.16a,b,c ; Table 3.2). Several CraA variants showed susceptibility towards certain drugs. Y42A, Y346A and F372A were susceptible towards ethidium and norfloxacin, I250A and R347A towards the phenicols and norfloxacin, L131A and L279A towards the phenicols and chlorhexidine, M70A towards florfenicol and norfloxacin, M247A only towards ethidium (Figure 3.16d; Table 3.2). Many CraA variants chosen to be studied (S135A, Y139A, M158A, S162A, L163A, F342A, K357A, L368A, M369A) did not show obvious impact on the drug susceptibility of the cells. Interestingly, L163A, L246A, Y268A and L279A were shown to be hyperactive on norfloxacin (Figure 3.16d; Table 3.2). 
a.

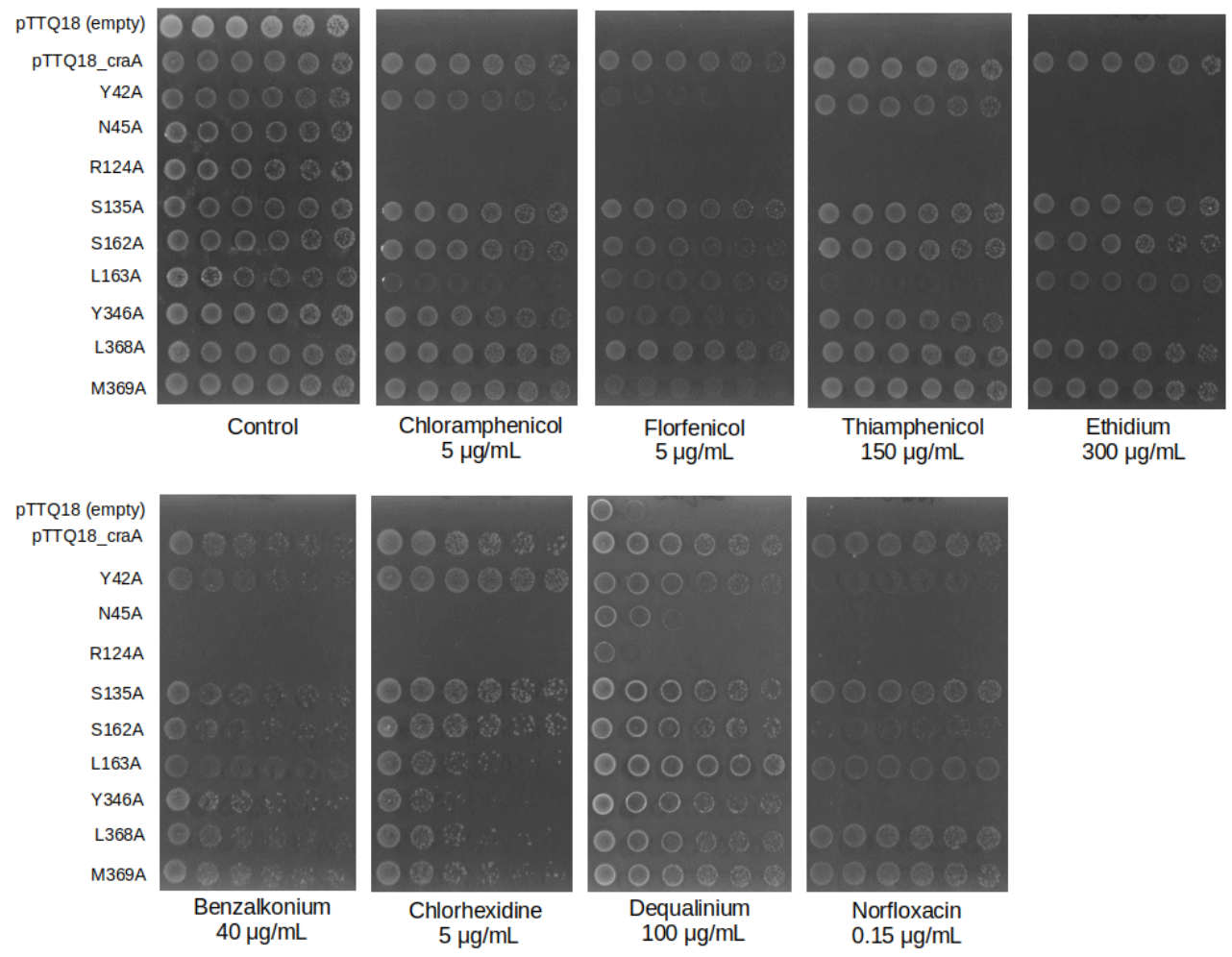

b.

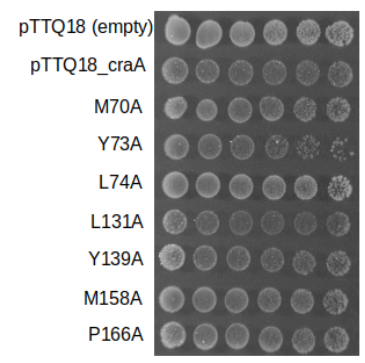

Control

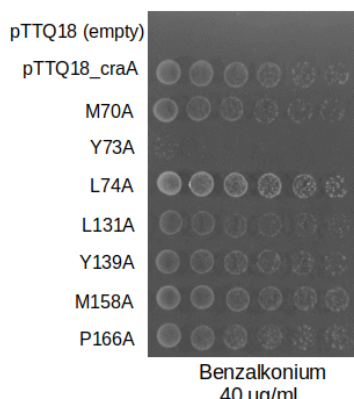

Benzalkonium

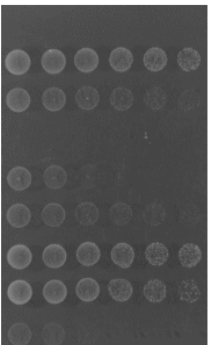

Chloramphenicol $5 \mu \mathrm{g} / \mathrm{mL}$

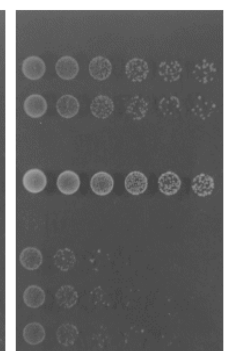

Chlorhexidine

Chlorhexidin
$5 \mu \mathrm{g} / \mathrm{mL}$

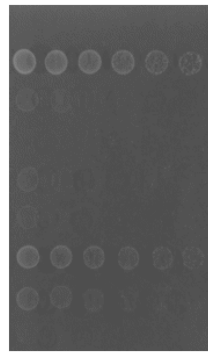

Florfenicol $5 \mu \mathrm{g} / \mathrm{mL}$

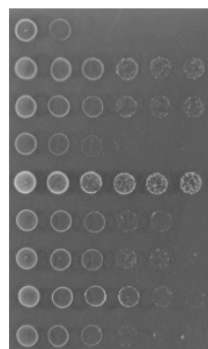

Dequalinium

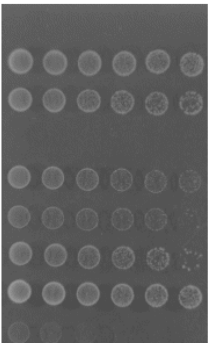

Thiamphenicol $100 \mu \mathrm{g} / \mathrm{mL}$

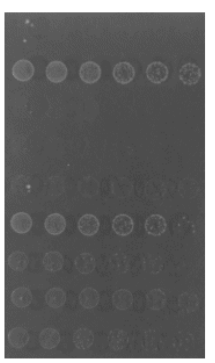

Norfloxacin

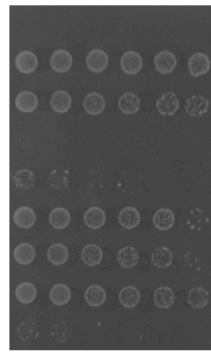

Ethidium

$300 \mu \mathrm{g} / \mathrm{mL}$

Figure 3.16 to be continued in the next page 
c.
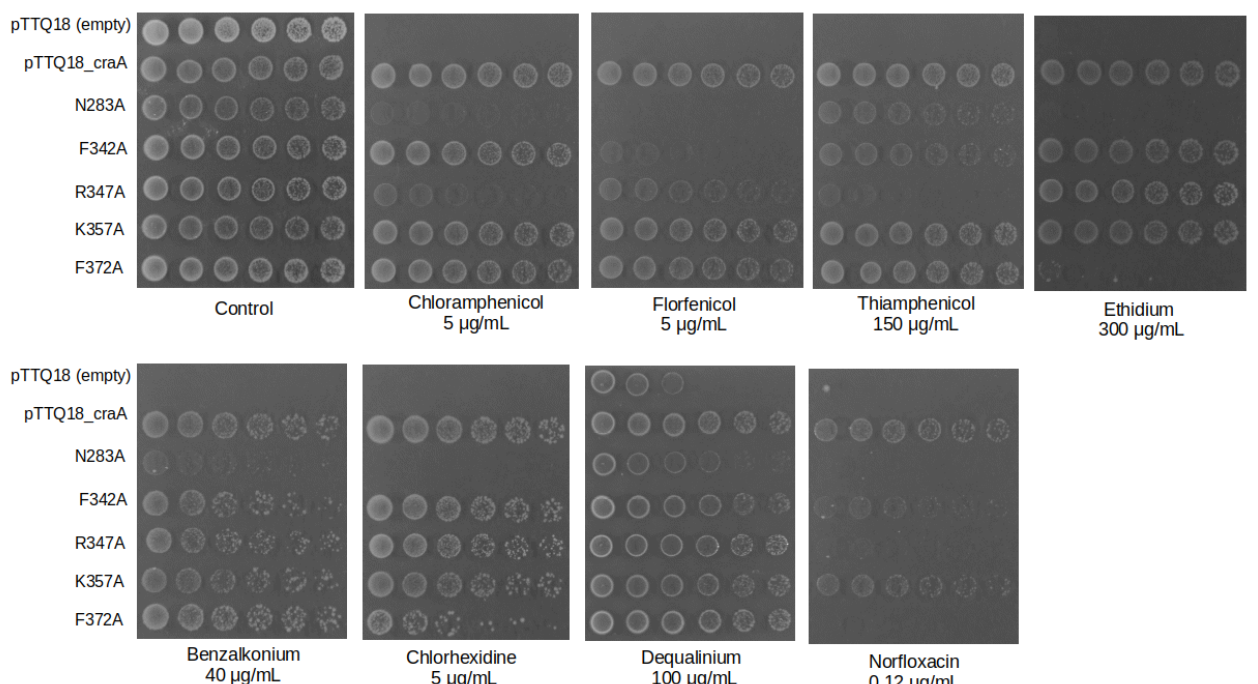

$5 \mu \mathrm{g} / \mathrm{mL}$

Dequalinium
$100 \mu \mathrm{g} / \mathrm{mL}$

Norfloxacin

$300 \mu \mathrm{g} / \mathrm{mL}$

d.
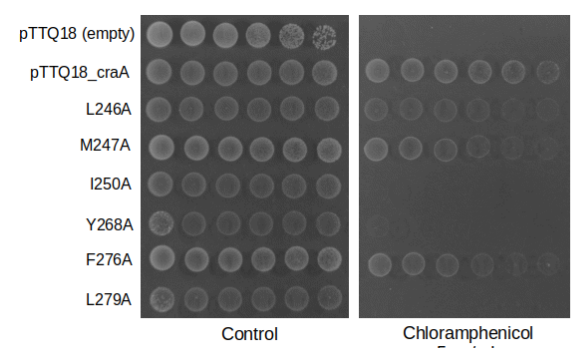

Chloramphenic
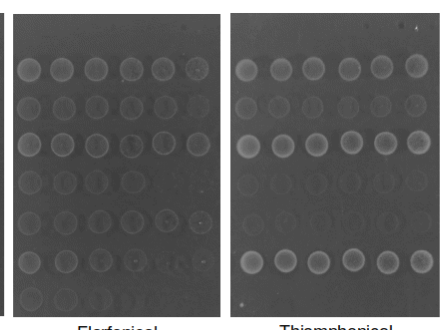

Florfenicol
$5 \mu \mathrm{g} / \mathrm{mL}$

Thiamphenico
$100 \mu g / \mathrm{mL}$
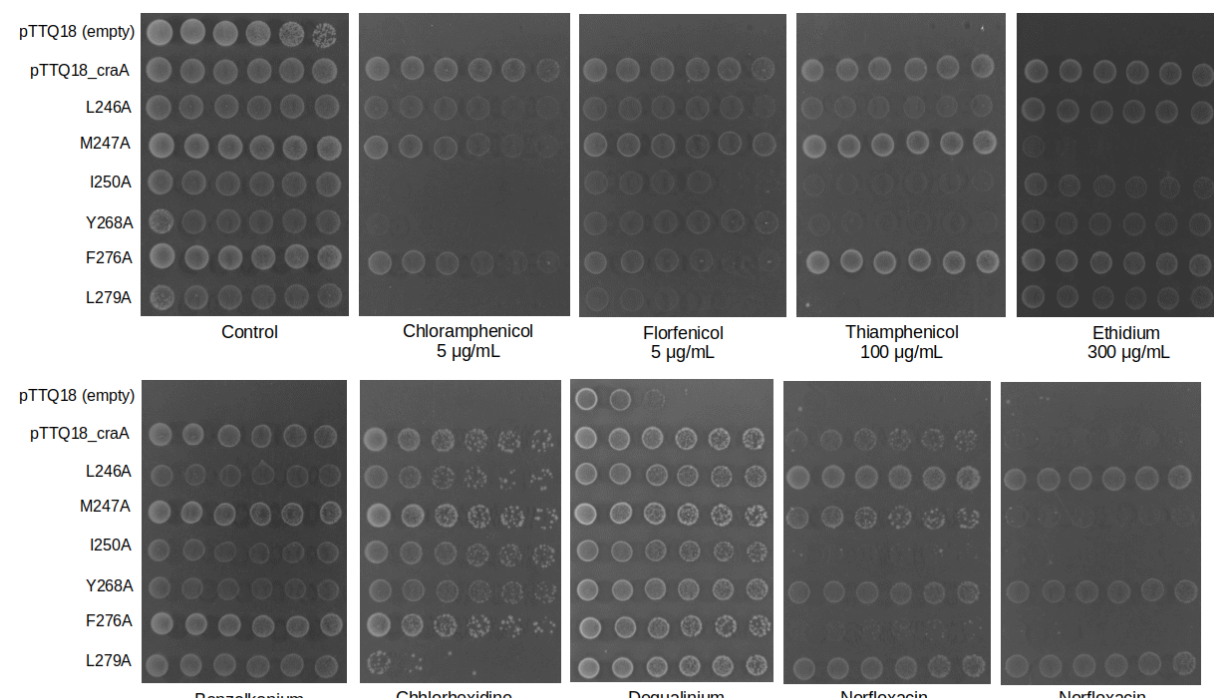

Benzalkonium

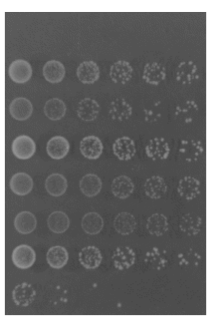

Chhlorhexidine
$5 \mu \mathrm{g} / \mathrm{mL}$

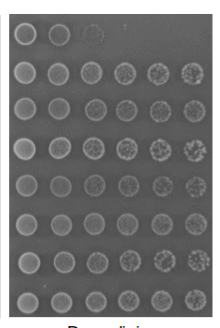

Dequalinium

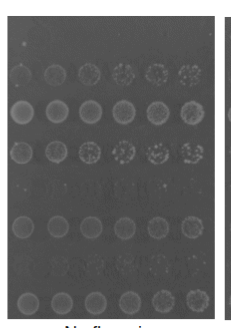

Norfloxacin

$300 \mu \mathrm{g} / \mathrm{mL}$

Figure 3.16 a,b,c,d Drug susceptibility assays of E. coli BW25113 $\Delta$ emrE $\Delta m d f A$ harbouring pTTQ18_craA and $c r a A$ variants. Serial diluted cell cultures harbouring empty vector (pTTQ18) or pTTQ18_craA (WT or variants) were spotted on LB agar plates supplemented with $0.2 \mathrm{mM} \mathrm{IPTG}, 50 \mu \mathrm{g} / \mathrm{mL}$ carbenicillin and the indicated drugs (drugs and concentration given below the plate figures). Cells harbouring empty vector pTTQ18 are included as a negative control. Experiments were conducted at least three times and the results shown here are representative. 


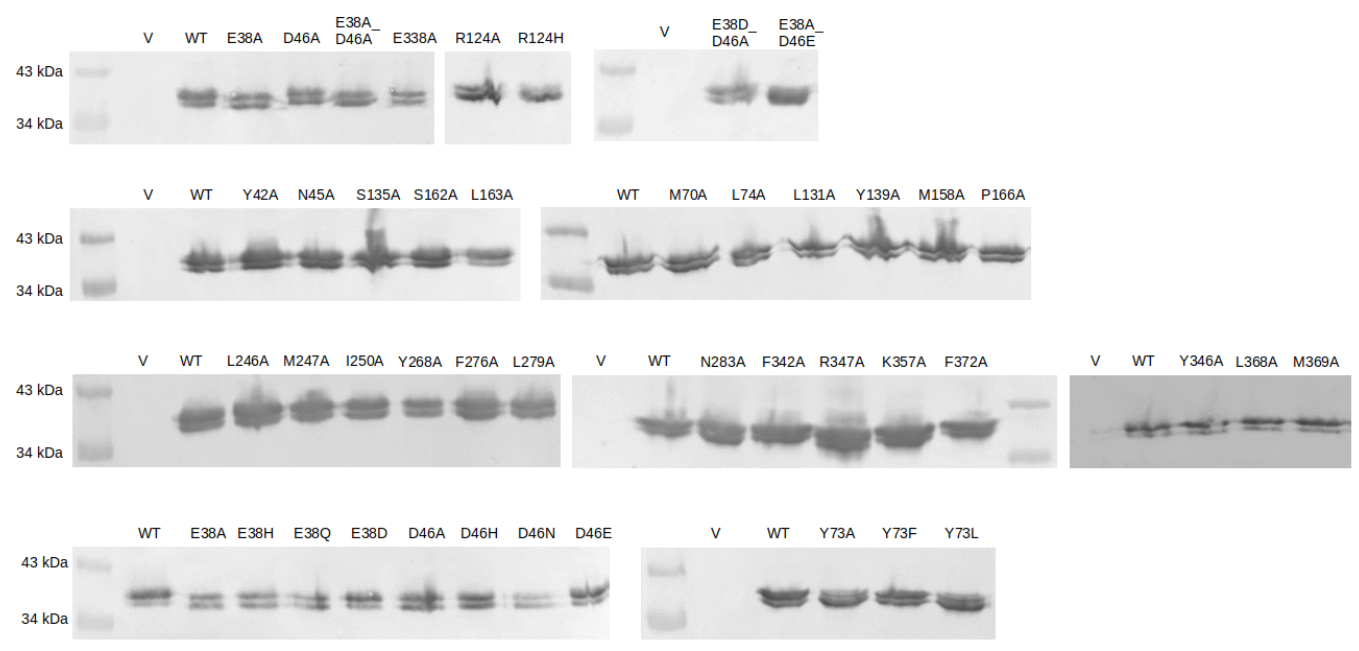

Figure 3.17 Levels of expression of craA wild-type and variants (whole cell extracts). Western blot analysis using anti-His antibody and anti-Mouse antibody conjugated with alkaline phosphatase showed that all craA variants were expressed at the similar level compared to the wild-type.

Table 3.2 Drug susceptibility profiles of CraA variants. Data shown are mean \pm standard error of mean, $n \geq 4$. * represents $P=0.005-0.05$ and ${ }^{* *}$ represents $P<0.005$ (Student's T-test).

\begin{tabular}{|c|c|c|c|c|c|c|c|c|}
\hline \multirow[b]{2}{*}{ Variants } & \multicolumn{8}{|c|}{ Substrates and concentrations } \\
\hline & $\begin{array}{l}\text { Chloramphenicol } \\
2.5-5 \mu \mathrm{g} / \mathrm{mL}\end{array}$ & $\begin{array}{l}\text { Florfenicol } \\
2.5-5 \mu \mathrm{g} / \mathrm{mL}\end{array}$ & $\begin{array}{l}\text { Thiamphenicol } \\
20 \mu \mathrm{g} / \mathrm{mL}\end{array}$ & $\begin{array}{l}\text { Ethidium } \\
100 \mu \mathrm{g} / \mathrm{mL}\end{array}$ & $\begin{array}{l}\text { Benzalkonium } \\
30-40 \mu \mathrm{g} / \mathrm{mL}\end{array}$ & $\begin{array}{l}\text { Chlorhexidine } \\
2.5-5 \mu \mathrm{g} / \mathrm{mL}\end{array}$ & $\begin{array}{l}\text { Dequalinium } \\
100-200 \mu \mathrm{g} / \mathrm{mL}\end{array}$ & $\begin{array}{c}\text { Norfloxacin } \\
0.10-0.15 \mu \mathrm{g} / \mathrm{mL}\end{array}$ \\
\hline WT & $1 \pm 0$ & $1 \pm 0$ & $1 \pm 0$ & $1 \pm 0$ & $1 \pm 0$ & $1 \pm 0$ & $1 \pm 0$ & $1 \pm 0$ \\
\hline E38A & $0.042 \pm 0.042$ ** & $0 \pm 0$ & $0.125 \pm 0.080$ ** & $0 \pm 0$ & $0 \pm 0$ & $0 \pm 0$ & $0.283 \pm 0.032$ ** & $0 \pm 0$ \\
\hline E38H & $0.042 \pm 0.042$ ** & $0 \pm 0$ & $0.167 \pm 0.118^{* *}$ & $0 \pm 0$ & $0.083 \pm 0.083^{* *}$ & $0 \pm 0$ & $0.317 \pm 0.032^{* *}$ & $0 \pm 0$ \\
\hline E38Q & $0.792 \pm 0.069$ * & $0.396 \pm 0.151^{\star *}$ & $1 \pm 0$ & $0 \pm 0$ & $0.708 \pm 0.105^{*}$ & $0 \pm 0$ & $0.344 \pm 0.011^{\star *}$ & $0 \pm 0$ \\
\hline E38D & $0.063 \pm 0.063$ ** & $0 \pm 0$ & $1 \pm 0$ & $0 \pm 0$ & $1 \pm 0$ & $0 \pm 0$ & $0.456 \pm 0.052$ ** & $0 \pm 0$ \\
\hline D46A & $0.5 \pm 0.1136$ *夫 & $0.958 \pm 0.027$ & $0.208 \pm 0.125$ ** & $0 \pm 0$ & $0.667 \pm 0.118^{*}$ & $0 \pm 0$ & $0.344 \pm 0.011$ ** & $1 \pm 0$ \\
\hline $\mathrm{D} 46 \mathrm{H}$ & $0.167 \pm 0.10^{* *}$ & $0.229 \pm 0.083^{* *}$ & $0.167 \pm 0.118^{* *}$ & $0 \pm 0$ & $0.583 \pm 0.144$ * & $0 \pm 0$ & $0.2 \pm 0.027^{\text {**}}$ & $0.327 \pm 0.093^{\star *}$ \\
\hline $\mathrm{D} 46 \mathrm{~N}$ & $0.188 \pm 0.102^{\star \star}$ & $0.896 \pm 0.0625$ & $1 \pm 0$ & $0 \pm 0$ & $1 \pm 0$ & $0 \pm 0$ & $0.372 \pm 0.028^{* *}$ & $1.044 \pm 0.0229$ \\
\hline D46E & $0.021 \pm 0.021$ ** & $0.542 \pm 0.108^{* *}$ & $0.167 \pm 0.118^{* *}$ & $1 \pm 0$ & $1 \pm 0$ & $1 \pm 0$ & $0.911 \pm 0.059$ & $0.133 \pm 0.133^{* *}$ \\
\hline E38A D46A & $0 \pm 0$ & $0 \pm 0$ & $0 \pm 0$ & $0 \pm 0$ & $0 \pm 0$ & $0 \pm 0$ & $0.22 \pm 0.056$ & N/A \\
\hline E38D D46A & $0.042 \pm 0.027^{* *}$ & $0.125 \pm 0.061$ ** & $0 \pm 0$ & $0 \pm 0$ & $0 \pm 0$ & $0 \pm 0$ & $0.256 \pm 0.035^{* *}$ & $0 \pm 0$ \\
\hline D46E E38A & $0 \pm 0$ & $0.021 \pm 0.021$ ** & $0 \pm 0$ & $0 \pm 0$ & $0 \pm 0$ & $0 \pm 0$ & $0.228 \pm 0.034^{* *}$ & $0 \pm 0$ \\
\hline
\end{tabular}

\begin{tabular}{|l|c|c|c|c|c|c|c|c|}
\hline & \multicolumn{9}{|c|}{ Substrates and concentrations } \\
\hline Variants & $\begin{array}{c}\text { Chloramphenicol } \\
5 \mu \mathrm{g} / \mathrm{mL}\end{array}$ & $\begin{array}{c}\text { Florfenicol } \\
5 \mu \mathrm{g} / \mathrm{mL}\end{array}$ & $\begin{array}{c}\text { Thiamphenicol } \\
20 \mu \mathrm{g} / \mathrm{mL}\end{array}$ & $\begin{array}{c}\text { Ethidium } \\
300 \mu \mathrm{g} / \mathrm{mL}\end{array}$ & $\begin{array}{c}\text { Benzalkonium } \\
30-40 \mu \mathrm{g} / \mathrm{mL}\end{array}$ & $\begin{array}{c}\text { Chlorhexidine } \\
5 \mu \mathrm{g} / \mathrm{mL}\end{array}$ & $\begin{array}{c}\text { Dequalinium } \\
120-150 \mu \mathrm{g} / \mathrm{mL}\end{array}$ & $\begin{array}{c}\text { Norfloxacin } \\
0.12-0.15 \mu \mathrm{g} / \mathrm{mL}\end{array}$ \\
\hline WT & $1 \pm 0$ & $1 \pm 0$ & $1 \pm 0$ & $1 \pm 0$ & $1 \pm 0$ & $1 \pm 0$ & $1 \pm 0$ \\
\hline Y73A & $0 \pm 0$ & $0 \pm 0$ & $0 \pm 0$ & $0 \pm 0$ & $0.148 \pm 0.076 *$ & $0.342 \pm 0.114^{* *}$ & $0.6 \pm 0.085^{* *}$ & $0 \pm 0$ \\
\hline Y73F & $1 \pm 0$ & $1 \pm 0$ & $1 \pm 0$ & $1 \pm 0$ & $1.05 \pm 0.05$ & $1.05 \pm 0.05$ & $1 \pm 0$ \\
\hline Y73L & $0 \pm 0$ & $0 \pm 0$ & $0 \pm 0$ & $0 \pm 0$ & $0 \pm 0$ & $0 \pm 0$ & $0.333 \pm 0.0966^{* *}$ & $0 \pm 0$ \\
\hline R124A & $0 \pm 0$ & $0 \pm 0$ & $0 \pm 0$ & $0 \pm 0$ & $0 \pm 0$ & $0 \pm 0$ & $0.167 \pm 0$ & $0 \pm 0$ \\
\hline R124H & $0 \pm 0$ & $0 \pm 0$ & $0 \pm 0$ & $0 \pm 0$ & $0 \pm 0$ & $0 \pm 0$ & $0.167 \pm 0$ & $0 \pm 0$ \\
\hline
\end{tabular}

Table 3.2 to be continued in the next page 
Results

\begin{tabular}{|c|c|c|c|c|c|c|c|c|}
\hline \multirow[b]{2}{*}{ Variants } & \multicolumn{8}{|c|}{ Substrates and concentrations } \\
\hline & $\begin{array}{c}\text { Chloramphenicol } \\
5 \mu \mathrm{g} / \mathrm{mL}\end{array}$ & $\begin{array}{l}\text { Florfenicol } \\
5 \mu \mathrm{g} / \mathrm{mL}\end{array}$ & $\begin{array}{l}\text { Thiamphenicol } \\
20 \mu \mathrm{g} / \mathrm{mL}\end{array}$ & $\begin{array}{l}\text { Ethidium } \\
300 \mu \mathrm{g} / \mathrm{mL}\end{array}$ & $\begin{array}{l}\text { Benzalkonium } \\
30-40 \mu \mathrm{g} / \mathrm{mL}\end{array}$ & $\begin{array}{l}\text { Chlorhexidine } \\
5 \mu \mathrm{g} / \mathrm{mL}\end{array}$ & $\begin{array}{c}\text { Dequalinium } \\
120-150 \mu \mathrm{g} / \mathrm{mL}\end{array}$ & $\begin{array}{c}\text { Norfloxacin } \\
0.12-0.15 \mu \mathrm{g} / \mathrm{mL}\end{array}$ \\
\hline WT & $1 \pm 0$ & $1 \pm 0$ & $1 \pm 0$ & $1 \pm 0$ & $1 \pm 0$ & $1 \pm 0$ & $1 \pm 0$ & $1 \pm 0$ \\
\hline Y42A & $0.944 \pm 0.035$ & $0.567 \pm 0.041 * *$ & $1 \pm 0$ & $0 \pm 0$ & $0.940 \pm 0.039$ & $1 \pm 0$ & $1 \pm 0$ & $0.53 \pm 0.121^{\star \star}$ \\
\hline N45A & $0 \pm 0$ & $0 \pm 0$ & $0 \pm 0$ & $0 \pm 0$ & $0.071 \pm 0.050$ ** & $0 \pm 0$ & $0.625 \pm 0.082^{\star *}$ & $0 \pm 0$ \\
\hline$M 70 A$ & $0.83 \pm 0$ ** & $0.37 \pm 0.122^{\star \star}$ & $1 \pm 0$ & $0.917 \pm 0.048$ & $0.94 \pm 0.056$ & $1 \pm 0$ & $0.97 \pm 0.033$ & $0 \pm 0$ \\
\hline L74A & $0.50 \pm 0.096$ ** & $0.33 \pm 0.105^{\star \star}$ & $0.56 \pm 0.147^{*}$ & $0.167 \pm 0.068^{* *}$ & $1 \pm 0$ & $1.13 \pm 0.125$ & $1 \pm 0$ & $0.33 \pm 0.096$ ** \\
\hline L131A & $0.79 \pm 0.042^{* *}$ & $0.13 \pm 0.062$ ** & $0.67 \pm 0.096^{*}$ & $1 \pm 0$ & $0.94 \pm 0.056$ & $0.083 \pm 0.083^{* *}$ & $1 \pm 0$ & $1 \pm 0$ \\
\hline S135A & $1 \pm 0$ & $1 \pm 0$ & $1 \pm 0$ & $1 \pm 0$ & $0.940 \pm 0.039$ & $1 \pm 0$ & $1 \pm 0$ & $1.25 \pm 0.181$ \\
\hline Y139A & $1 \pm 0$ & $1 \pm 0$ & $1 \pm 0$ & $1 \pm 0$ & $1 \pm 0$ & $0.40 \pm 0.115^{* *}$ & $1 \pm 0$ & $0.375 \pm 0.125^{* \star}$ \\
\hline M158A & $1 \pm 0$ & $0.67 \pm 0.139$ * & $1 \pm 0$ & $0.71 \pm 0.197$ & $1 \pm 0$ & $0.33 \pm 0.152^{\star \star}$ & $1 \pm 0$ & $0.417 \pm 0.160$ * \\
\hline S162A & $1 \pm 0$ & $0.967 \pm 0.033$ & $1 \pm 0$ & $0.972 \pm 0.028$ & $0.940 \pm 0.039$ & $0.944 \pm 0.056$ & $1 \pm 0$ & $0.379 \pm 0.110^{* *}$ \\
\hline L163A & $0.556 \pm 0.102^{\star *}$ & $1 \pm 0$ & $0.146 \pm 0.080$ ** & $1 \pm 0$ & $1.071 \pm 0.071$ & $0.5 \pm 0.096$ * & $1 \pm 0$ & $1.545 \pm 0.450$ \\
\hline P166A & $0.083 \pm 0.048$ ** & $0.1 \pm 0.067^{* \star}$ & $0.11 \pm 0.111$ ** & $0.21 \pm 0.158^{* *}$ & $1 \pm 0$ & $0.52 \pm 0.098^{* \star}$ & $0.90 \pm 0.10$ & $0.625 \pm 0.042 * *$ \\
\hline L246A & $0.944 \pm 0.056$ & $1 \pm 0$ & $1 \pm 0$ & $1 \pm 0$ & $1 \pm 0$ & $1 \pm 0$ & $0.89 \pm 0.069$ & $1 \pm 0$ \\
\hline M247A & $0.944 \pm 0.056$ & $1 \pm 0$ & $1 \pm 0$ & $0.167 \pm 0.096$ ** & $1 \pm 0$ & $1 \pm 0$ & $0.93 \pm 0.067$ & $1 \pm 0$ \\
\hline $\mathrm{I} 250 \mathrm{~A}$ & $0 \pm 0$ & $0.67 \pm 0.068^{* *}$ & $0.61 \pm 0.056 *$ & $0.583 \pm 0.068^{* *}$ & $1 \pm 0$ & $1 \pm 0$ & $0.89 \pm 0.069$ & $0 \pm 0$ \\
\hline Y268A & $0.444 \pm 0.147^{*}$ & $0.79 \pm 0.125$ & $0.61 \pm 0.056$ ** & $1 \pm 0$ & $1 \pm 0$ & $1 \pm 0$ & $1.24 \pm 0.193$ & $1 \pm 0$ \\
\hline F276A & $1 \pm 0$ & $0.71 \pm 0.125$ & $1 \pm 0$ & $1 \pm 0$ & $1 \pm 0$ & $1 \pm 0$ & $0.97 \pm 0.086$ & $0 \pm 0$ \\
\hline L279A & $0.167 \pm 0.096$ ** & $0.417 \pm 0.048$ * & $0 \pm 0$ & $1 \pm 0$ & $1 \pm 0$ & $0.33 \pm 0.075^{* *}$ & $1.04 \pm 0.04$ & $1 \pm 0$ \\
\hline N283A & $0.306 \pm 0.10^{* *}$ & $0 \pm 0$ & $0.833 \pm 0.043^{* *}$ & $0 \pm 0$ & $0.513 \pm 0.125^{* *}$ & $0 \pm 0$ & $0.75 \pm 0.108$ & $0 \pm 0$ \\
\hline E338A & $0.267 \pm 0.113^{* *}$ & $0.361 \pm 0.067^{* *}$ & $0.389 \pm 0.056$ ** & $0.5 \pm 0.18^{*}$ & $0.917 \pm 0.083$ & $0 \pm 0$ & $0.625 \pm 0.042^{* *}$ & $0 \pm 0$ \\
\hline F342A & $1 \pm 0$ & $0.389 \pm 0.035^{\star *}$ & $0.944 \pm 0.035$ & $0.944 \pm 0.035$ & $1 \pm 0$ & $1 \pm 0$ & $1 \pm 0$ & $0.833 \pm 0.075$ \\
\hline Y346A & $1 \pm 0$ & $0.6 \pm 0.1^{* *}$ & $0.938 \pm 0.030^{* *}$ & $0 \pm 0$ & $0.833 \pm 0.089$ & $0.444 \pm 0.056$ ** & $0.948 \pm 0.035$ & $0.045 \pm 0.045^{\star *}$ \\
\hline R347A & $0.50 \pm 0.043^{* *}$ & $0.778 \pm 0.035^{* *}$ & $0.333 \pm 0.075^{* *}$ & $1 \pm 0$ & $1 \pm 0$ & $1 \pm 0$ & $1 \pm 0$ & $0.5 \pm 0.149$ * \\
\hline K357A & $1 \pm 0$ & $1 \pm 0$ & $1 \pm 0$ & $1 \pm 0$ & $1 \pm 0$ & $0.96 \pm 0.04$ & $1 \pm 0$ & $1 \pm 0$ \\
\hline L368A & $1 \pm 0$ & $1 \pm 0$ & $1 \pm 0$ & $0.972 \pm 0.028$ & $0.976 \pm 0.024$ & $0.5 \pm 0.096$ * & $0.969 \pm 0.031$ & $1.273 \pm 0.183$ \\
\hline M369A & $1 \pm 0$ & $0.667 \pm 0.075^{* *}$ & $1 \pm 0$ & $1 \pm 0$ & $1 \pm 0$ & $0.444 \pm 0.147^{*}$ & $1 \pm 0$ & $1.106 \pm 0.10$ \\
\hline F372A & $1 \pm 0$ & $0.944 \pm 0.056$ & $1 \pm 0$ & $0.083 \pm 0.037$ ** & $1.1 \pm 0.1$ & $0.54 \pm 0.123$ * & $0.958 \pm 0.042$ & $0.233 \pm 0.194$ ** \\
\hline
\end{tabular}

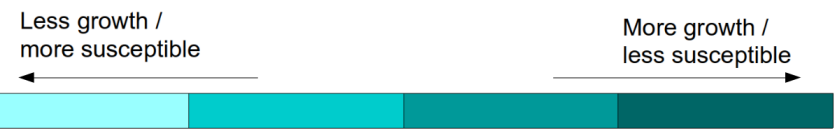

\subsubsection{Dequalinium transport in whole cells}

Through drug susceptibility assays, the dicationic antiseptic agent dequalinium was identified as one of the CraA substrates (Figure 3.10 ; Table 3.2). The fluorescence property of dequalinium enables the transport and accumulation of this substrate to be followed in an assay which detects the dequalinium fluorescence. In the transport assay, the incubation of cells with buffers supplemented with $2 \mu \mathrm{M}$ dequalinium leads to accumulation of dequalinium into the cells. The accumulation of dequalinium causes fluorescence quenching, while dequenching occurs when dequalinium is removed from the cells. The fluorescence remained unchanged for $E$. coli BW25113 $\Delta$ emrE $\Delta m d f A \Delta a c r A B$ expressing wild-type craA, 
implying that dequalinium was constantly removed from the cells (Figure 3.18a). In contrast, dequalinium was accumulated in the cells harbouring empty vector, as evidenced by fluorescence quenching. Alanine-substitution of E38, D46 or E338 resulted in fluorescence quenching, indicating the inactivity of these variants (Figure 3.18a). When chloramphenicol was added in addition to dequalinium, the removal of dequalinium from the cells overexpressing craA was impeded, as evidenced by the fluorescence quenching (Figure $3.18 \mathrm{~b})$, implying that chloramphenicol is a competitive substrate.

a.

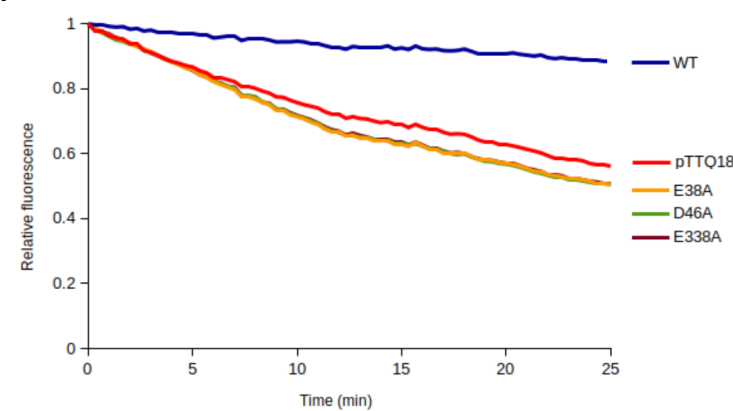

b.

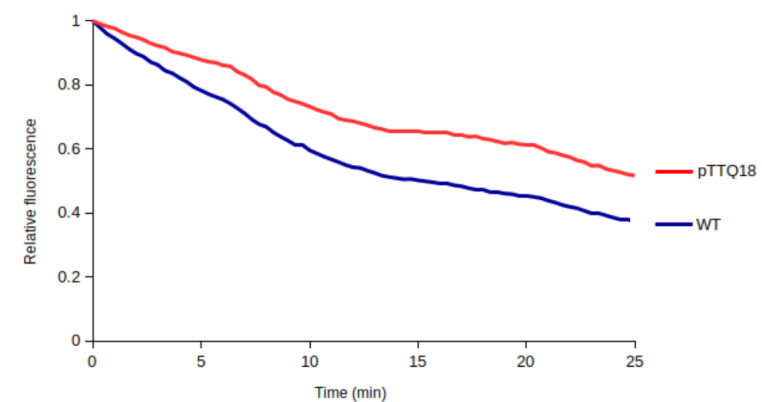

Figure 3.18 Dequalinium transport assay in whole cell conducted using E. coli BW25113 $\triangle$ emrE $\triangle m d f A \triangle a c r A B$ overexpressing $\mathrm{craA}$ wild-type or variants. (a) The negative control (cells harbouring empty vector pTTQ18), $\mathrm{E} 38 \mathrm{~A}, \mathrm{D} 46 \mathrm{~A}$ and E338A incubated with $2 \mu \mathrm{M}$ of dequalinium showed quenched fluorescence as the dequalinium entered the cells. WT maintained the dequalinium fluorescence, indicating that dequalinium was extruded continuously. (b) When $1 \mathrm{mM}$ of chloramphenicol was additionally added as a competitor, both WT and negative control experienced fluorescence quenching, implying that chloramphenicol is a competitive substrate.

To identify whether the quenching of fluorescence is due to the accumulation of dequalinium in the cells, the dequalinium loaded cells were lysed with SDS. A remarkable increase of the fluorescence was observed for cells harbouring empty vector or CraA variants after the cells were incubated with dequalinium for 15 minutes as compared to the cells without dequalinium incubation (0 minute) (Figure 3.19). In contrast, only a slight increase of fluorescence was observed for cells overexpressing wild-type craA upon lysis. This indicates that the intracellular accumulation of dequalinium in the cells was prevented during the 15minute incubation. 


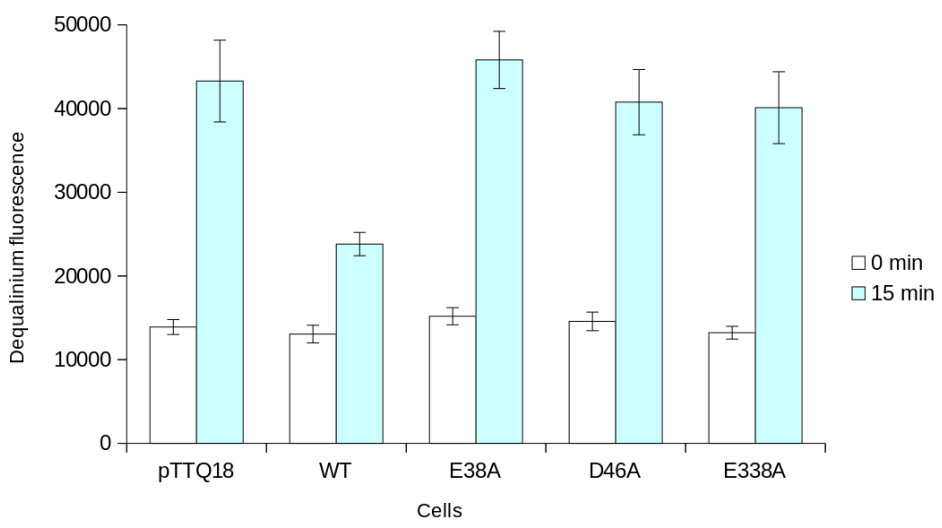

Figure 3.19 Accumulation of dequalinium in E. coli BW25113 $\triangle$ emrE $\triangle m d f A \triangle a c r A B$ harbouring pTTQ18, pTTQ18_CraA wild-type and variants. Substantial increase of fluorescence was observed for cells harbouring pTTQ18 (empty vector), E38A, D46A and E338A after incubation of cells with $2 \mu \mathrm{M}$ of dequalinium for 15 minutes. In contrast, accumulation of dequalinium for cells expressing wild-type $c r a A$ is relatively less, indicating a constant efflux of dequalinium from the cells. Experiments were conducted at least three times and error bar represents s.e.m. The difference in dequalinium accumulation between WT and other CraA variants was statistically significant ( $p$ value $<0.005$, Student's T-test).

\subsubsection{Ethidium efflux in whole cells}

Ethidium has been shown to be one of the CraA substrates (Figure 3.10 ; Table 3.2). It has fluorescence properties which can be exploited in a continuous fluorescence assay. The fluorescence of ethidium is significantly enhanced when it is bound to DNA and the fluorescence is quenched upon removal of ethidium from the cells. E. coli BW25113 $\triangle e m r E \Delta m d f A$ harbouring empty vector (pTTQ18), pTTQ18_craA_WT, or the craA variants were incubated with $10 \mu \mathrm{M}$ ethidium in the absence of energy source and the presence of an efflux inhibitor carbonyl cyanide m-chlorophenylhydrazine (CCCP) that dissipates the proton motive force. After removal of CCCP by washing the cells, reenergization of cells by addition of glucose causes a rapid decrease of fluorescence for craA_WT-expressing cells, corresponding to the active efflux of ethidium out of the cells. In contrast, ethidium fluorescence remained unchanged for the cells harbouring empty vector (Figure 3.20 right panel). When no glucose was added to reenergize the cells, no fluorescence quenching was observed for both cells (pTTQ18 or WT) (Figure 3.20 left panel). 

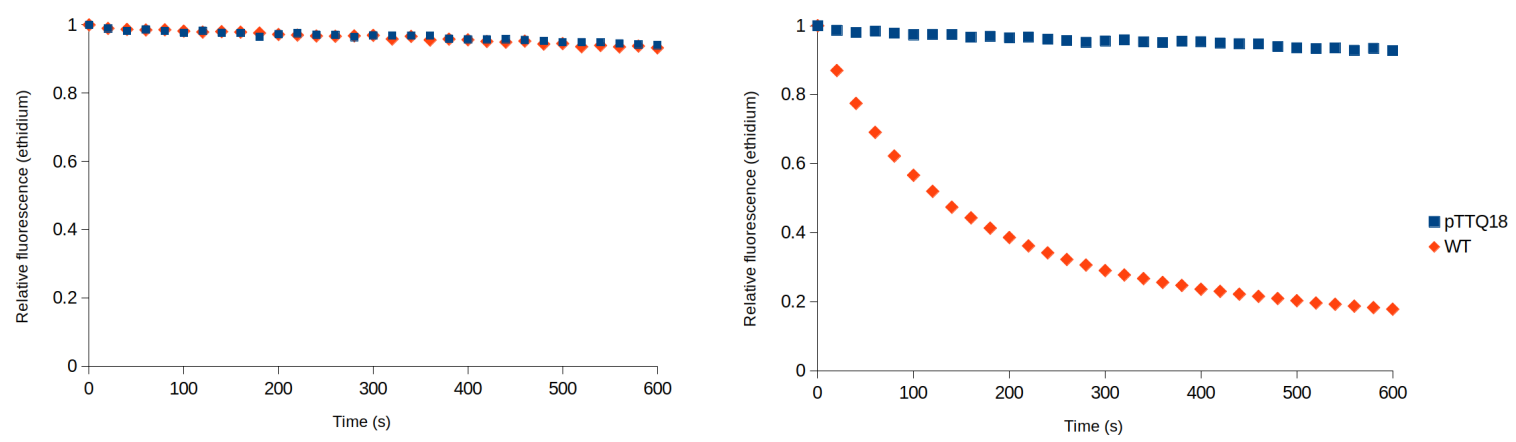

Figure 3.20 Ethidium efflux catalyzed by CraA. Assay was conducted using E. coli BW25113 $\triangle$ emrEAmdfA harbouring empty vector (pTTQ18) or wild-type CraA incubated with $10 \mu \mathrm{M}$ ethidium and $40 \mu \mathrm{M}$ CCCP. Left panel: Experiment without glucose added. Right panel: Reenergization by adding $0.36 \%$ glucose causes cells overexpressing craA to remove ethidium from the cells. The fluorescence signal of negative control (pTTQ18) remained unchanged. Experiments were conducted at least three times and the results shown here are representative.

Based on the results of the drug susceptibility assays (Table 3.2) and molecular docking experiment (Figure 3.34), several CraA variants predicted to be important in the binding and transport of ethidium were selected for the ethidium efflux assay. Fluorescence of ethidium did not decrease substantially for E38A, N45A, N283A, R124A, and R124H, indicating that these variants are lack or have considerably diminished ethidium efflux activity (Figure 3.21 left panel). In contrast, alanine substitution of some residues (L131A, L246A, Y268A and $\mathrm{M} 369 \mathrm{~A}$ ) did not affect the activity of CraA in ethidium efflux (Figure 3.21 right panel). This is in accordance to the observation in the drug susceptibility assays which also showed that these variants were not susceptible to ethidium (Table 3.2). 

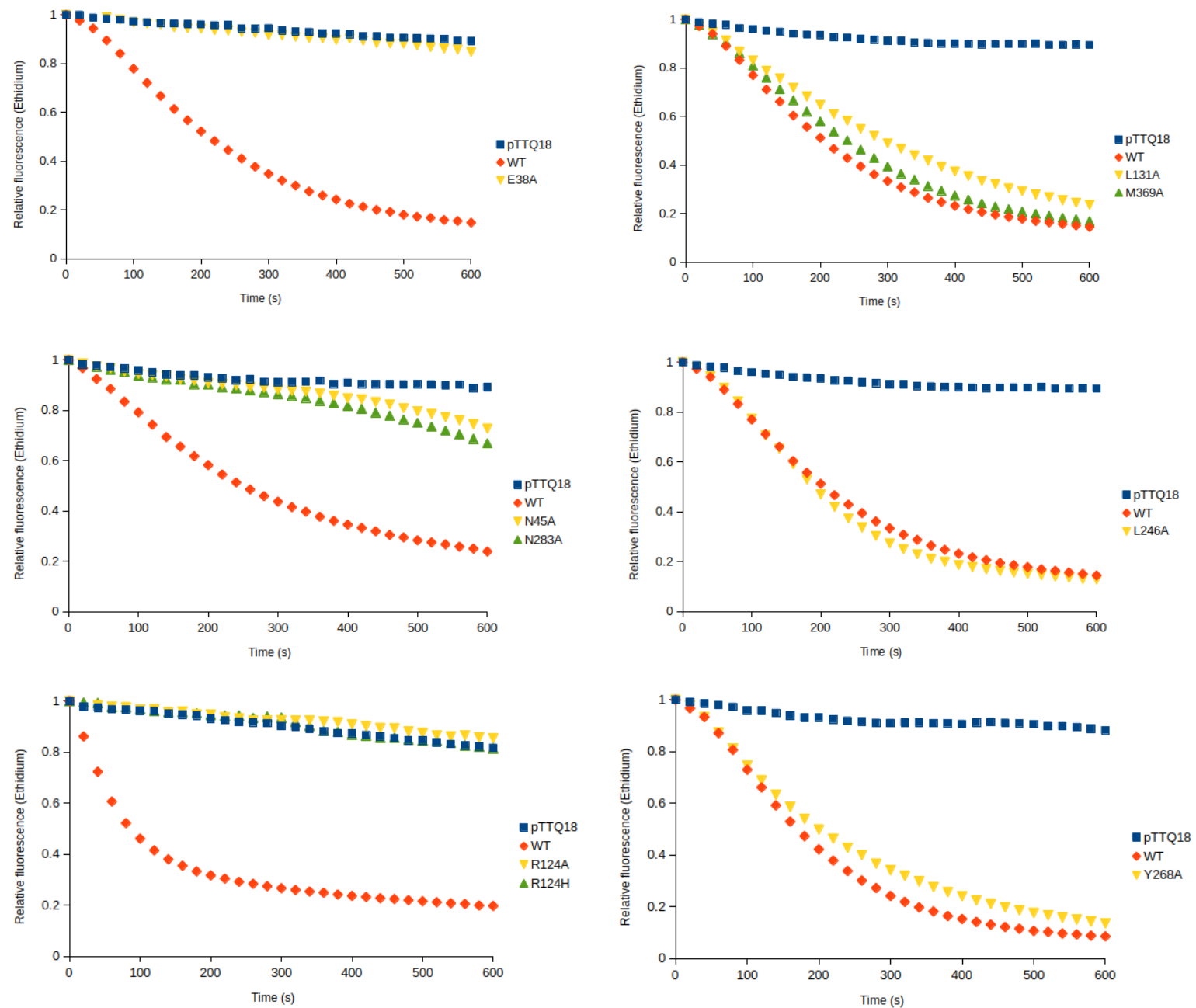

Figure 3.21 Ethidium efflux catalyzed by CraA and variants. Assay was conducted using E. coli BW25113 $\triangle e m r E \Delta m d f A$ harbouring empty vector (pTTQ18), wild-type CraA (WT) or CraA variants incubated with $10 \mu \mathrm{M}$ ethidium. Left panel: E38A, N45A, N283A, R124A and R124H were inactive in ethidium efflux. Right panel: L131A, M369A, L246A and Y268A were active in ethidium efflux. Experiments were conducted at least three times and the results shown here are representative. 
Several CraA variants (L74A, M158A, I250A and E338A) showed intermediate ethidium efflux activity (Figure 3.22 ), comparable to the results from drug susceptibility assay (Table $3.2)$.
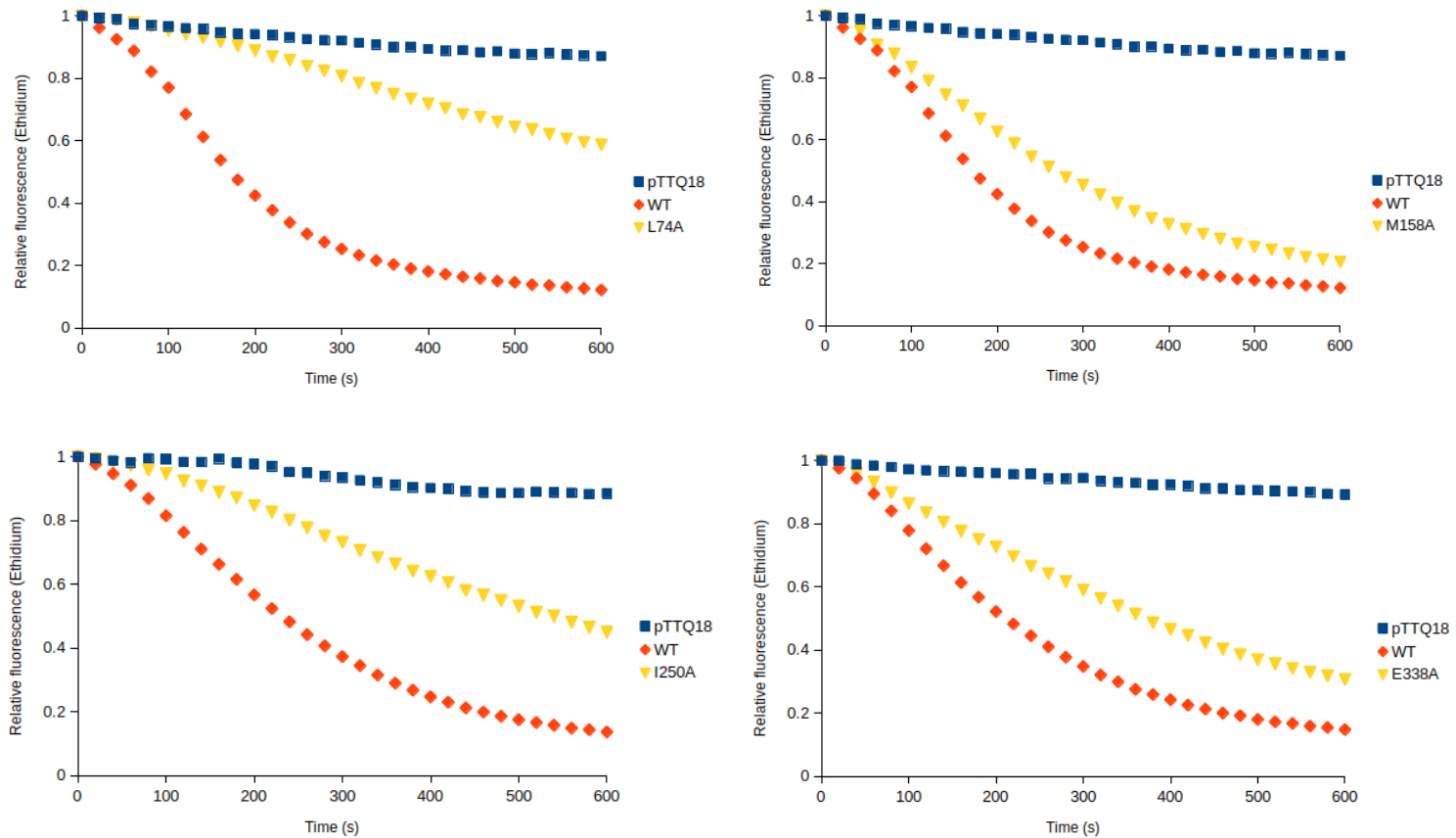

Figure 3.22 Ethidium efflux catalyzed by CraA and variants. Assay was conducted using E. coli BW25113 $\triangle$ emrEsmdfA harbouring empty vector (pTTQ18), wild-type CraA (WT) or CraA variants. L74A, M158A, I250A and E338A showed intermediate efflux activity in comparison to WT. Experiments were conducted at least three times and the results shown here are representative.

However, not all variants displayed a consistence phenotype between susceptibility (growth) assays and ethidium efflux assay. Cells expressing craA_Y42A,craA_D46A, craA_Y346A, and craA_F372A were active or partially active in ethidium efflux (Figure 3.23), but the growth of the cells expressing these variants was retarded in drug susceptibility assay (Table $3.2)$.

The ethidium efflux assay revealed two CraA variants (L163A and L279A) that were hyperactive, i.e. the fluorescence signal reduced slightly faster than the WT (Figure 3.24). Substitution of tyrosine at position 73 to alanine abolished the function of CraA in ethidium efflux, but substitution to phenylalanine resulted in wild-type CraA activity (Figure 3.25). 

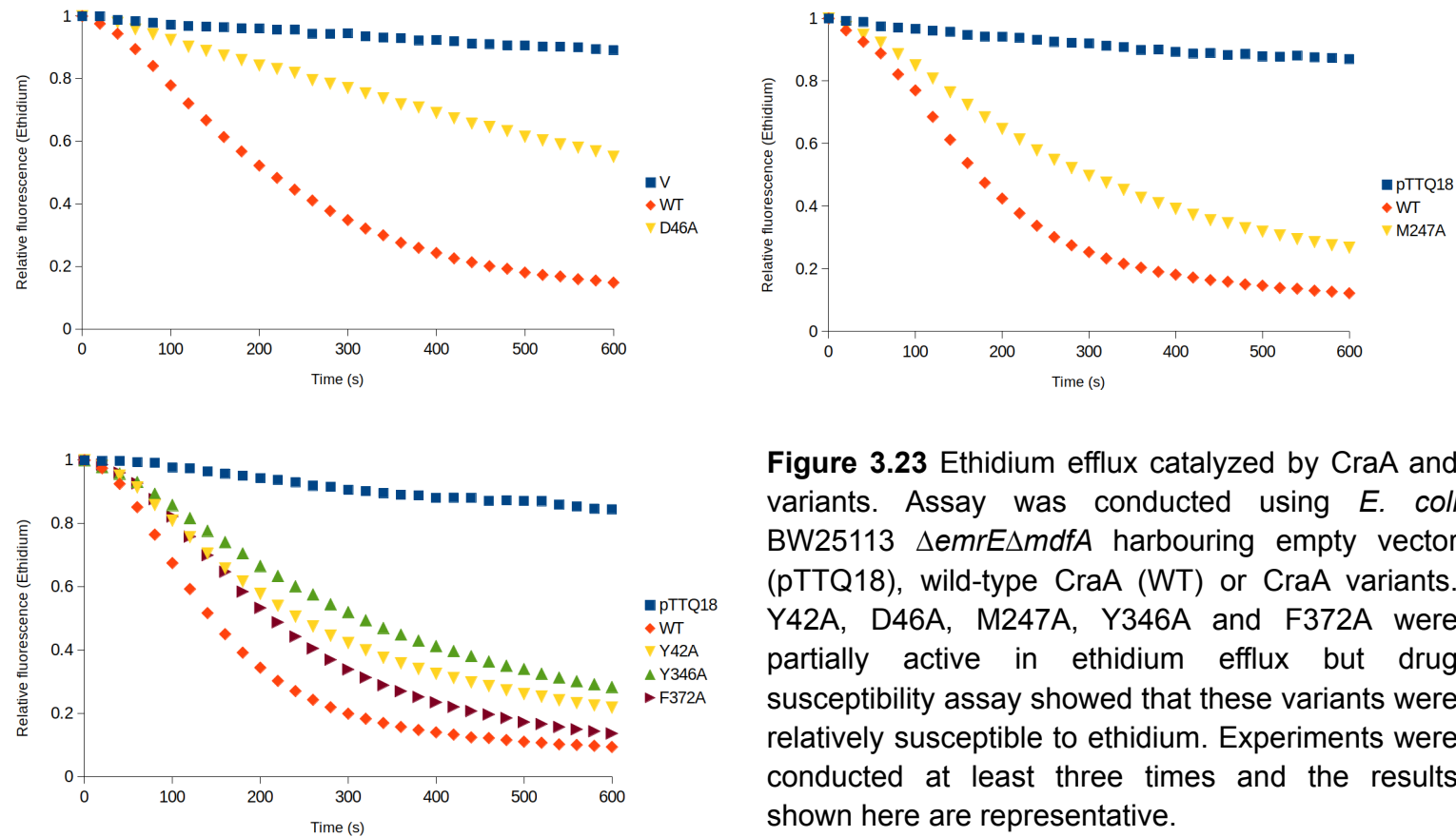

Figure 3.23 Ethidium efflux catalyzed by CraA and variants. Assay was conducted using $E$. coli BW25113 $\triangle$ emrE $\Delta m d f A$ harbouring empty vector (pTTQ18), wild-type CraA (WT) or CraA variants. Y42A, D46A, M247A, Y346A and F372A were partially active in ethidium efflux but drug susceptibility assay showed that these variants were relatively susceptible to ethidium. Experiments were conducted at least three times and the results shown here are representative.
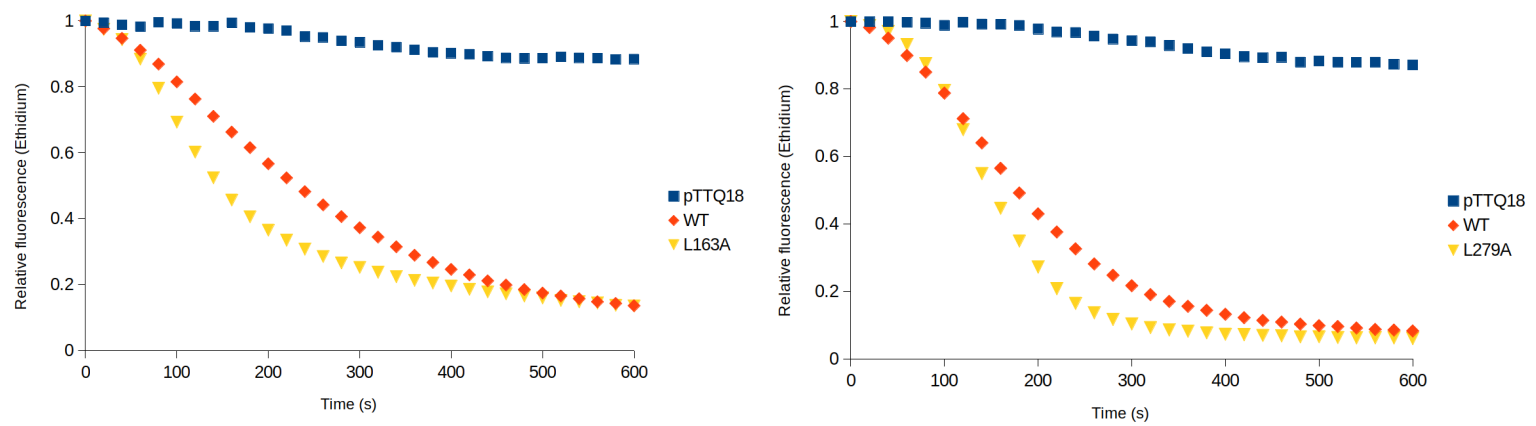

Figure 3.24 Ethidium efflux catalyzed by CraA and variants. Assay was conducted using E. coli BW25113 $\Delta$ emrE $\Delta m d f A$ harbouring empty vector pTTQ18 (V), wild-type CraA (WT) or CraA variants. L163A and L279A showed hyperactivity in ethidium efflux. Experiments were conducted at least three times and the results shown here are representative.

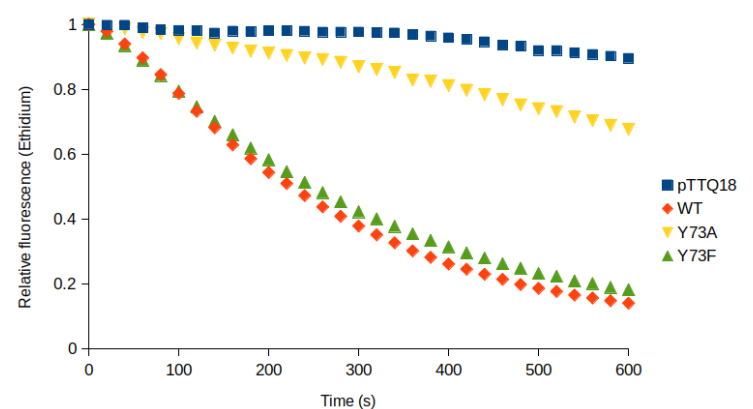

Figure 3.25 Ethidium efflux catalyzed by CraA and Y73 variants. Assay was conducted using E. coli BW25113 $\triangle$ emrEAmdfA harbouring empty vector (pTTQ18), wild-type CraA (WT) or Y73 variants. Y73A was inactive in ethidium efflux in comparison to WT but Y73F was active. Experiments were conducted at least three times and the result shown here is representative. 


\subsubsection{Norfloxacin accumulation assay}

Through drug susceptibility assays, it was shown that fluoroquinolones such as norfloxacin and ciprofloxacin are substrates of CraA (Figure 3.10). To further validate this hypothesis, the amount of norfloxacin accumulated in E. coli BW25113 $\Delta$ emrE $\Delta m d f A$ overexpressing wild-type craA or variants was determined after five minutes of exposure to $100 \mu \mathrm{g} / \mathrm{mL}$ of norfloxacin. Cells harbouring empty vector (pTTQ18) were used as negative control.

It was clearly shown that the cells harbouring empty vector (pTTQ18) accumulated higher amount of norfloxacin compared to the cells overexpressing wild-type cra $A$, as indicated by the higher fluorescence intensity of norfloxacin (Figure 3.26, 3.27, 3.28). In general, the results obtained via the accumulation assay are comparable to the results from the drug susceptibility assay. CraA variants which conferred more accumulation of norfloxacin than WT are shown in Figure 3.26, in which the detected fluorescence is higher than the cells harbouring wild-type craA. The variants E38A, N45A, N283A and E338A showed even more norfloxacin accumulation compared to cells harbouring empty vector (Figure 3.26). Interestingly, several variants showed a hyperactive phenotype for norfloxacin efflux, i.e these variants accumulated less norfloxacin compared to the WT (Figure 3.27).

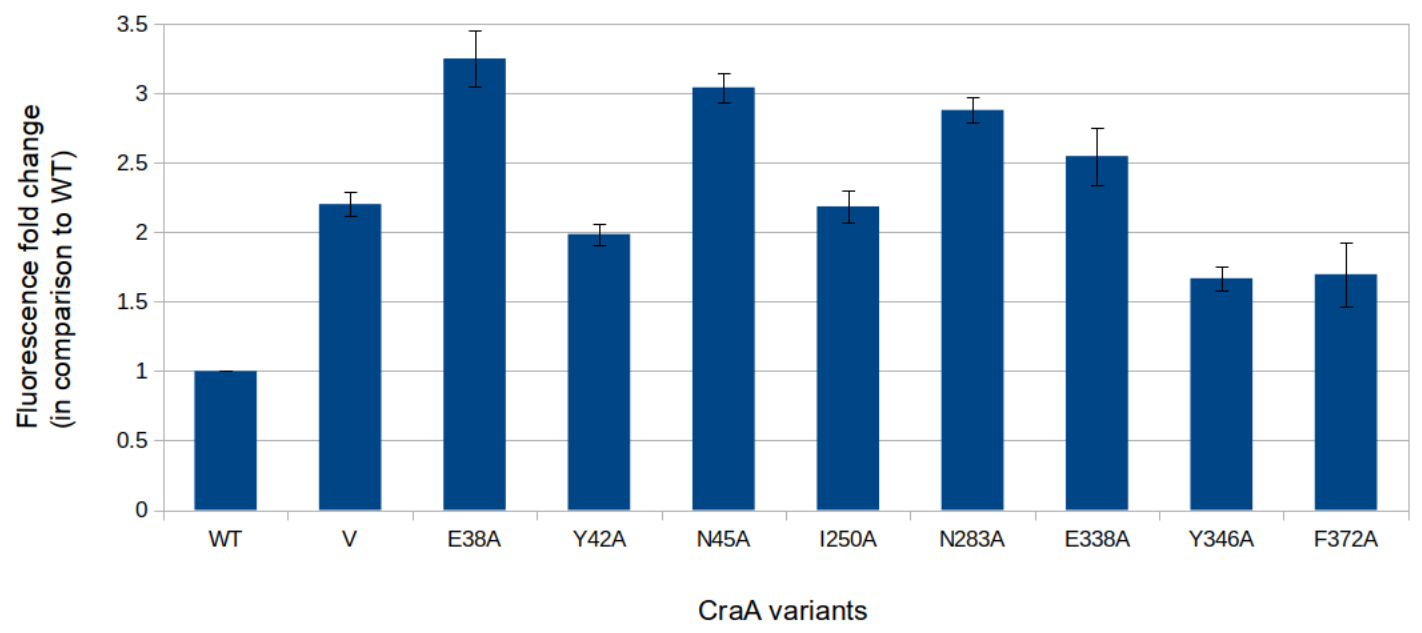

Figure 3.26 Norfloxacin accumulation assay (susceptible variants). The assay was conducted using $E$. coli BW25113 $\Delta$ emrE $\Delta m d f A$ harbouring empty vector pTTQ18 (V), wild-type CraA (WT) or CraA variants. Cells were incubated with $100 \mu \mathrm{g} / \mathrm{mL}$ norfloxacin for 5 minutes before measurement. E38A, Y42A, N45A, I250A, N283A, E338A, Y346A and F372A accumulated more norfloxacin than the WT. Experiments were conducted at least four times and the error bars represent the s.e.m. 


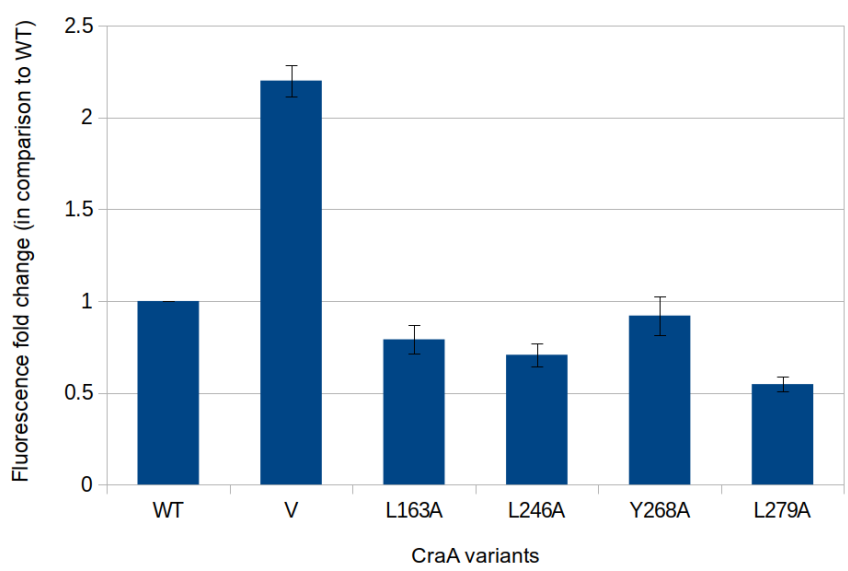

Figure 3.27 Norfloxacin accumulation assay (hyperactive variants). The assay was conducted using E. coli BW25113 $\Delta$ emrE $\Delta m d f A$ harbouring empty vector pTTQ18 (V), wild-type CraA (WT) or CraA variants. Cells were incubated with $100 \mu \mathrm{g} / \mathrm{mL}$ norfloxacin for 5 minutes before measurement. L163A, L246A, Y268A and L279A were hyperactive in norfloxacin efflux, as they accumulated less norfloxacin than the WT. Experiments were conducted at least four times and the error bars represent the s.e.m.

Whereas Y73A or Y73A compromised norfloxacin efflux, Y73F conferred wild-type CraA norfloxacin efflux activity. (Figure 3.28a). Similar to the results of the drug susceptibility assays, the norfloxacin accumulation assay also revealed that the D46A variant conferred still some degree of resistance against norfloxacin. It accumulates more norfloxacin than WT, but less than the empty vector $(\mathrm{V})$. D46N, on the other hand shows wild-type CraA norfloxacin efflux activity (Figure 3.28b).

a.

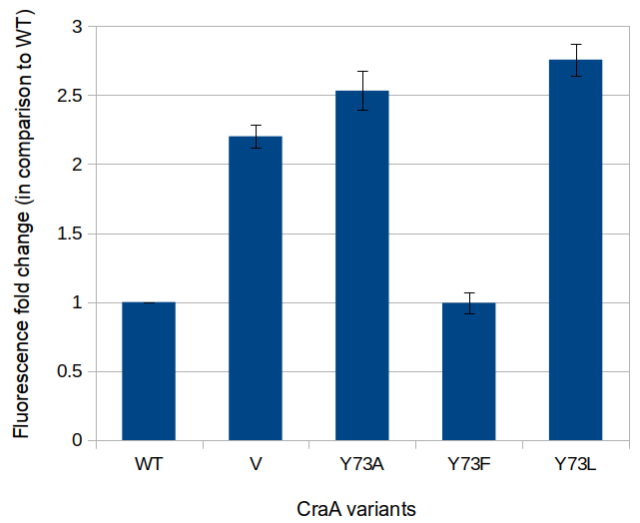

b.

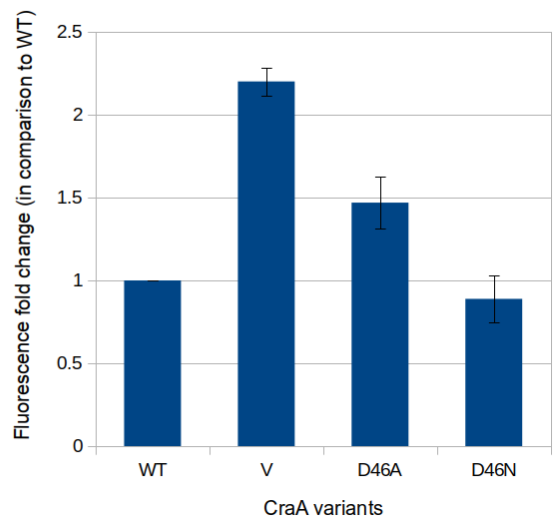

Figure 3.28 Norfloxacin accumulation assay (Y73 and D46 variants). The assay was conducted using E. coli BW25113 $\Delta$ emrE $\Delta m d f A$ harbouring empty vector pTTQ18 (V), wild-type CraA (WT) or CraA variants. Cells were incubated with $100 \mu \mathrm{g} / \mathrm{mL}$ norfloxacin for 5 minutes before measurement. (a) Y73A and Y73L are inactive in norfloxacin efflux, while Y73F is active as the WT. (b) D46A is less active in norfloxacin efflux while D46N is active. Experiments were conducted at least four times and the error bars represent the s.e.m. 


\subsubsection{Proton transport assay using inverted membrane vesicles}

To examine the CraA-mediated $\mathrm{H}^{+} /$drug antiport process, proton transport assay using $\Delta \mathrm{pH}$ sensitive fluorescence dye, 9-amino-6-chloro-2-methoxyacridine (ACMA) was conducted on inverted membrane vesicles containing wild-type CraA or each of the CraA variants. Electroneutral ACMA can move freely across the lipid bilayer and equilibrates to the same concentration. Upon addition of lactate to the outside of the inverted membrane vesicles, protons are transported into the interior of the membrane vesicles, causing an equilibrium shift towards protonated ACMA. The unprotonated ACMA inside the vesicles re-equilibrate via diffusion of unprotonated ACMA from the outside of the vesicles, causing an overall increase of ACMA (protonated+unprotonated) concentration inside the vesicles. The increased concentration of ACMA inside the vesicles leads to self-quenching (Figure 3.29). Addition of CraA substrates activate the efflux activity of the pump, in exchange for protons, which reduces the proton concentration and protonated ACMA concentration, resulting in dequenching of fluorescence signal. Addition of CCCP, a proton uncoupler, causes an increase of the fluorescence due to the dissipation of the $\Delta \mathrm{pH}$ and $\Delta \Psi$ (Figure 3.29).

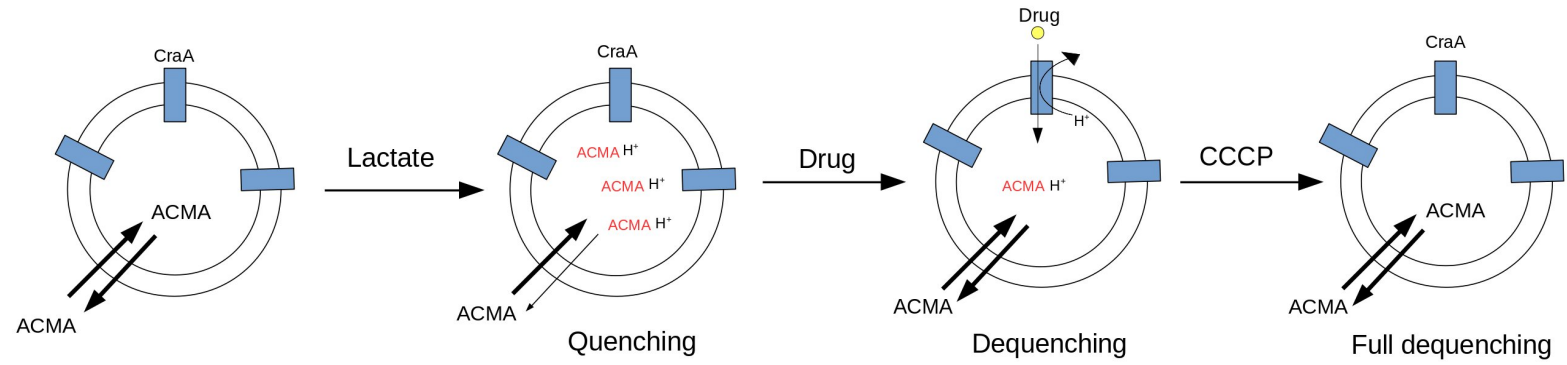

Figure 3.29 Illustration of proton transport assay conducted using inverted membrane vesicles prepared from $E$. coli BW25113 $\triangle$ emrE $\Delta m d f A \triangle a c r A B$ complemented with CraA. Lactate-driven proton pumping across the membrane establishes a transmembrane $\Delta \mathrm{pH}$ and $\Delta \psi$, causing interior acidification of the membrane vesicles as monitored by ACMA fluorescence quenching. Addition of CraA substrate causes a partial dissipation of the $\Delta \mathrm{pH}$ and $\Delta \Psi$ and results in dequenching of the ACMA fluorescence. Addition of CCCP causes further dequenching of ACMA fluorescence. 
Addition of dequalinium, $\mathrm{TPP}^{+}$or benzalkonium causes partial dissipation of the sensed $\Delta \mathrm{pH}$ across the membrane of CraA containing vesicles, leading to an increase in fluorescence (dequenching). For the control vesicles without $\mathrm{CraA}$ or inactive $\mathrm{CraA}$ variants, no dissipation of the $\Delta \mathrm{pH}$ was observed upon substrate addition, as indicated by the unchanged fluorescence signal. Addition of CCCP, a proton uncoupler, causes an increase of the fluorescence due to the dissipation of the $\Delta \mathrm{pH}$ (Figure 3.30 upper panel).

Experiments involving CraA variants E38A, D46A and E338A showed that the addition of dequalinium, TPP $^{+}$or benzalkonium to CraA variants containing vesicles causes either no change or a very small increase of fluorescence compared to the WT containing vesicles (Figure 3.30 lower panel). This indicates that E38A, D46A and E338A are less active in the transport of dequalinium, $\mathrm{TPP}^{+}$or benzalkonium compared to the WT.
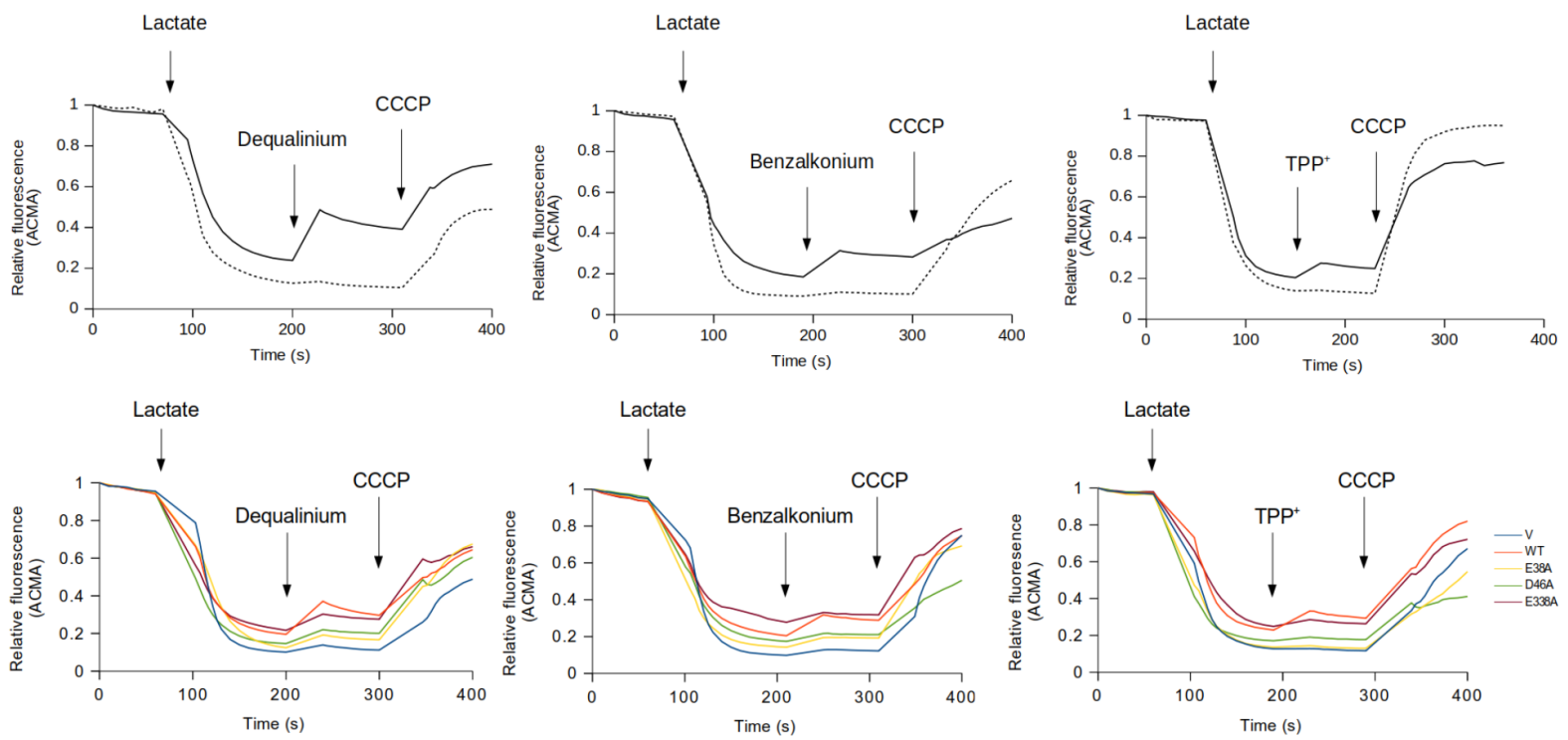

Figure 3.30 Proton-coupled substrate transport by inverted membrane vesicles. Upper panel: Addition of CraA substrate ( $50 \mu \mathrm{M}$ dequalinium, $0.03 \mu \mathrm{g} / \mathrm{mL}$ benzalkonium, or $250 \mu \mathrm{M} \mathrm{TPP}^{+}$) causes a partial dissipation of the $\Delta \mathrm{pH}$ in the CraA WWT (solid line) containing membrane vesicles while no fluorescence change was observed in control vesicles harbouring empty vector (dotted line). Lower panel: A small fluorescence increase or no fluorescence change was observed for CraA variants (E38A, D46A, E338A) upon addition of dequalinium, benzalkonium and $\mathrm{TPP}^{+}$, indicating a lower transport activity for these CraA variants. Concentrations of lactate and CCCP added were $2 \mathrm{mM}$ and $10 \mu \mathrm{M}$, respectively. Experiments were conducted at least three times and the results shown here are representative. 


\subsubsection{Phenotypic analysis of $A$. baumannii ATCC $19606 \Delta c r a A$ and complementation}

To confirm the role of CraA in $A$. baumannii, the craA gene was deleted from the chromosome of $A$. baumannii ATCC 19606 (described in 3.1) and the drug susceptibility of the $\triangle c r a A$ strain was determined. The drug agar plate assay showed that $A$. baumannii ATCC $19606 \triangle c r a A$ harbouring empty vector (pBAV1K) was susceptible to chloramphenicol, thiamphenicol, and florfenicol (Figure 3.31a). The complementation of CraA with plasmid pBAV1K_CraA restored resistance against these drugs, albeit to a lesser extent in comparison to wild-type $A$. baumannii ATCC 19606. In contrast to the results obtained with E. coli $\triangle e m r E \Delta m d f A, A$. baumannii ATCC $19606 \triangle c r a A$ remains resistant against benzalkonium, chlorhexidine and dequalinium (Figure 3.31b). This observation suggests that other efflux systems and/or the outer membrane impermeability might be dominant factors for the resistance against these drugs in A. baumannii.

a.

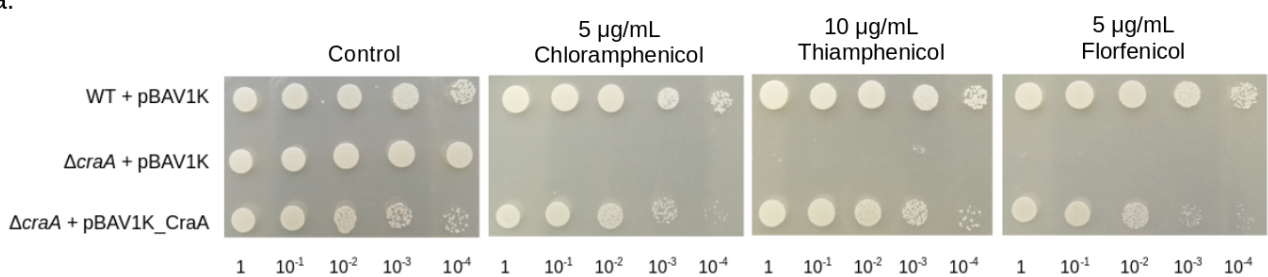

b.
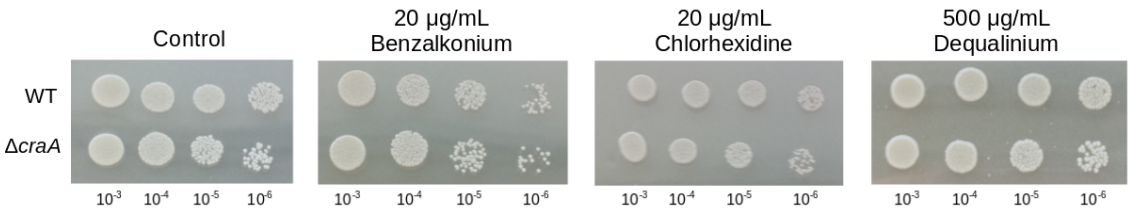

Figure 3.31 Drug susceptibility assays of wild-type $A$. baumannii ATCC 19606 (WT), $\Delta c r a A$ and $\Delta c r a A$ complemented with pBAV1K_CraA. (a) Diluted cultures (dilution given below the plate figures) were spotted on LB agar plates supplemented with $50 \mu \mathrm{g} / \mathrm{mL}$ kanamycin and the indicated phenicols (drugs and concentrations given above the plate figures). (b) Diluted cultures (dilution given below the plate figures) of WT and $\triangle c r a A$ spotted on LB agar plates supplemented with the indicated drugs (drugs and concentrations given above the plate figures) did not show sensitivity to the tested drugs. Experiments were conducted at least three times and the results shown here are representative. 


\subsection{Molecular docking of substrates in CraA}

The binding of chloramphenicol, ethidium, and norfloxacin to CraA was predicted through docking experiments using AutoDock Vina program (Trott and Olson 2010). In general, the results suggest that these drugs interact with CraA at the apex of the binding pocket, mainly through van der Waals interaction (Figure 3.32).
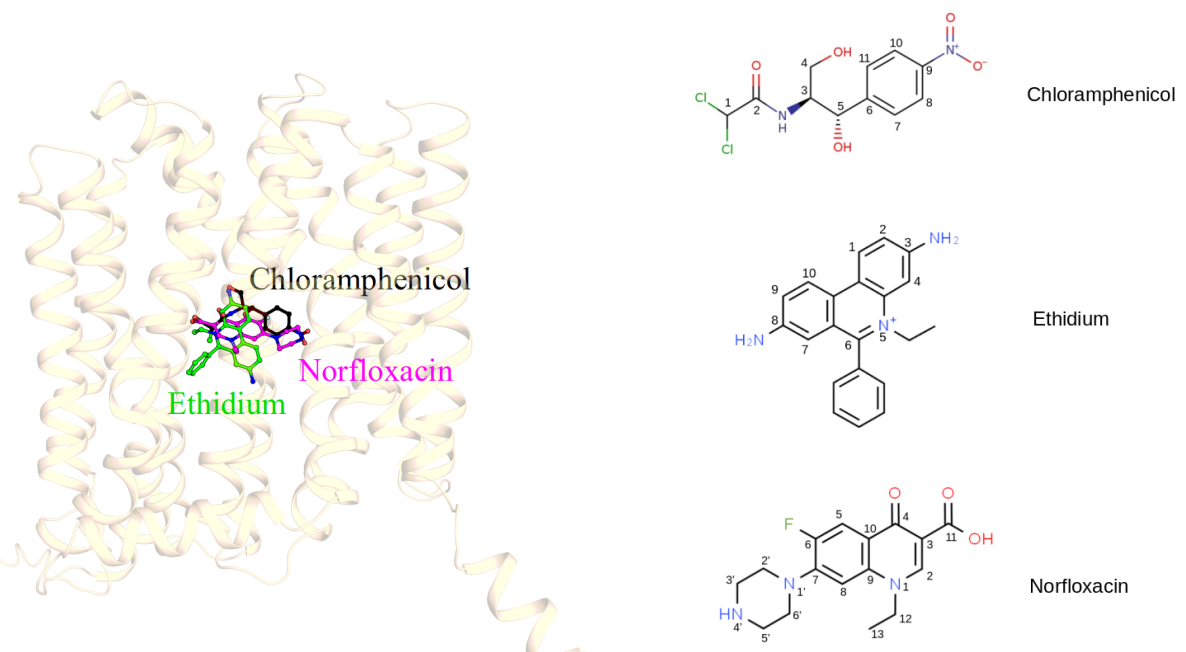

Figure 3.32 In silico analysis of chloramphenicol, ethidium or norfloxacin with CraA homology model. CraA model was obtained based on the MdfA-Q131A_L339A variant (PDB: 6EUQ) (Zomot et al., 2018). Molecular docking was performed using AutoDock Vina (Trott and Olson, 2010). Left: Docking solution for these molecules and CraA model indicates that these molecules bind to the apex of CraA binding pocket proximal to the periplasmic side. Right: Chemical structures and numbering scheme of chloramphenicol, ethidium, and norfloxacin.

\subsubsection{Docking of chloramphenicol}

Docking solution for chloramphenicol was superimposed with the crystal structure of MdfA (PDB: 4ZOW, Heng et al., 2015) in complex with chloramphenicol. The result shows that the binding of chloramphenicol to CraA agrees with the binding of chloramphenicol to MdfA (Figure 3.33a). Similar to MdfA, binding of chloramphenicol is mainly contributed by van der Waals interaction. Only three hydrogen bonds are observed for the interaction between chloramphenicol and CraA. More specifically, two hydrogen bonds are made via the carboxylate moeity of $\mathrm{D} 46$ with $\mathrm{C} 4-\mathrm{OH}$ and $\mathrm{C} 5-\mathrm{OH}$ of chloramphenicol, while the amino group of the carboxamide moeity of N45 interacts with $\mathrm{C} 2-\mathrm{O}$ of chloramphenicol (Figure $3.32,3.33 \mathrm{~b})$. The chloramphenicol dichloroacetamide moiety is surrounded by hydrophobic 
residues such as M70, Y73, L74, and L131. In contrast, hydrophobic residues like L246, M247, I250, F276, and L279 interact with the nitrobenzene moiety of chloramphenicol via van der Waals interaction. An exception is the residue E338 which is non-hydrophobic but it is in a defined rotamer state where the acyl group is facing the nitrobenzene moiety of chloramphenicol (Figure 3.33b). E38 located distal to chloramphenicol does not form any interaction with the drug molecule (Figure 3.33b).

Surprisingly, Y42 interacts with both the dichloroacetamide and nitrobenzene moiety of chloramphenicol by van der Waals interaction (Figure 3.33b). It was shown in drug susceptibility assays (Table 3.2) that alanine substitution of $Y 42$ rarely affect the resistance of CraA against chloramphenicol and thiamphenicol, except for florfenicol (Figure 3.33c). The reason for the discrepancy observed between docking solution for chloramphenicol and drug susceptibility assay is unclear. Unlike chloramphenicol and thiamphenicol, florfenicol possesses a fluorine instead of the $\mathrm{OH}$ group at position $\mathrm{C} 4$ (Figure 3.33c). It is speculated that the substitution of fluorine at position $\mathrm{C} 4$ prevents the formation of hydrogen bond with D46, which leads to slightly different drug binding pose in comparison to chloramphenicol or thiamphenicol, thereby facilitating a tighter interaction between $\mathrm{Y} 42$ and the dichloroacetamide moiety of florfenicol. Indeed, substitution of Y42 to alanine showed an increased susceptibility only against florfenicol (Figure 3.16a, Table 3.2).

a.

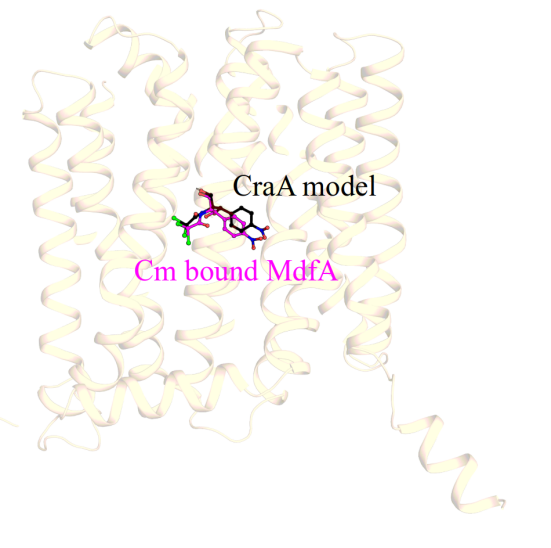

Figure 3.33 to be continued in the next page 
b.

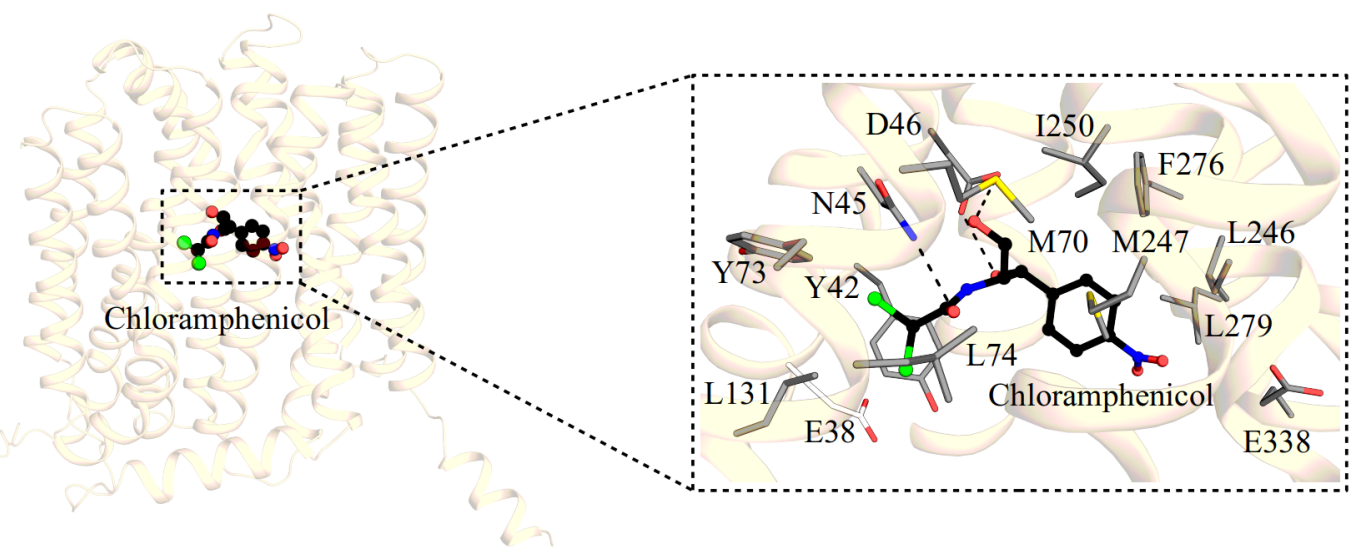

C.<smiles>O=C(NC(CO)C(O)c1ccc([N+](=O)[O-])cc1)C(Cl)Cl</smiles>

Chloramphenicol<smiles>CS(=O)(=O)c1ccc(C(O)C(CO)NC(=O)C(Cl)Cl)cc1</smiles>

Thiamphenicol<smiles>CS(=O)(=O)c1ccc(C(O)C(CF)NC(=O)C(Cl)Cl)cc1</smiles>

Florfenicol

Figure 3.33 In silico analysis of chloramphenicol binding to CraA. The CraA model was obtained based on the MdfA-Q131A_L339A variant (PDB: 6EUQ) (Zomot et al., 2018). Molecular docking was performed using AutoDock Vina (Trott and Olson, 2010). (a) Docking solution for chloramphenicol and CraA model agrees well with the chloramphenicol bound MdfA (PDB: 4ZOW) (Heng et al., 2015). (b) Docking solution for chloramphenicol and $\mathrm{CraA}$ model in the indicated region in (a), including the CraA side chain interactions. Putative hydrogen bonds are indicated with dashed lines. E38 (shown in white) does not interaction with chloramphenicol (Oxygen=red, nitrogen=blue, sulfur=yellow, chlorine=green). (c) Chemical structures of phenicols.

\subsubsection{Docking of ethidium}

Molecular docking for ethidium suggests that ethidium interacts with CraA binding pocket mainly through hydrophobic interactions. These residues include Y73, L74, L131, M247, and 1250. Additionally, Y42 and F372 interact with the ethidium diaminophenanthridinium moiety by $\pi$-stacking interaction. Interestingly, only residues N45 and D46 coordinate with the 3amino moiety of ethidium via hydrogen bonds (Figure 3.34). The docking solution was in-line with the drug susceptibility assay and ethidium transport assay, with the exception of L131A that showed no deleterious effect in both assays. Drug susceptibility assays showed that 
alanine substitution of M247 has reduced the resistance of CraA exclusively against ethidium (Figure 3.16d, Table 3.2). Ethidium transport assays indicated that M247A is slightly inactive in ethidium efflux. These results validate the docking solution for ethidium which shows that M247 plays a role in ethidium binding through hydrophobic interaction.

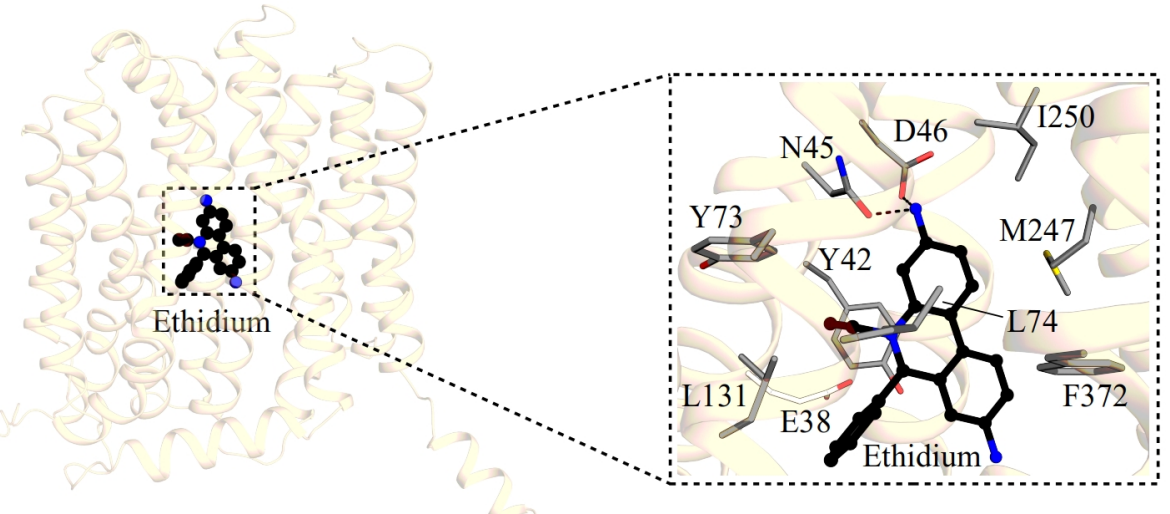

Figure 3.34 In silico analysis of ethidium binding to CraA. The CraA model was obtained based on the MdfAQ131A_L339A variant (PDB: 6EUQ) (Zomot et al., 2018). Molecular docking was performed using AutoDock Vina (Trott and Olson, 2010). Inlet shows the docking solution for ethidium and CraA model, including the CraA side chain interactions. Putative hydrogen bonds are indicated with dashed lines (Oxygen=red, nitrogen=blue, sulfur=yellow).

\subsubsection{Docking of norfloxacin}

Norfloxacin accumulation assays have confirmed that alanine-substitution of the residues E38, Y42, N45, D46, I250, N283, E338, Y346, and F372 reduced the efficiency of CraA in norfloxacin efflux (Figure 3.26). From the prediction of the norfloxacin binding via molecular docking, Y42 interacts with the quinoline group via $\pi-\pi$ interaction (further discussed in Chapter 4, section 4.2).

Only two hydrogen bonds were observed in the docking solution for norfloxacin. This includes residue N45 and D46, interacting with the carboxyl and carbonyl groups of norfloxacin, respectively (Figure $3.32,3.35$ ). Substitution of D46 to alanine reduced the efficiency of CraA in norfloxacin efflux, while $\mathrm{D} 46 \mathrm{~N}$ could rescue the activity to a level which is comparable to wild-type CraA (Figure 3.28b), indicating that the amino group of asparagine has the same function as the protonated hydroxyl group of aspartate. 
1250 forms intermolecular bonding with fluorine at C-6 position (Figure 3.32, 3.35). L246, F276, and L279 interact with the piperazine moiety via van der Waals interaction. F372 interacts with the $\mathrm{N}-1$ ethyl group via hydrophobic interaction (Figure 3.32, 3.35). E338 is positioned in proximity with the piperazine moiety, but it may still interact with the piperazine moiety during transport cycle (Figure 3.35). Cells expressing craA variant E338A accumulate more norfloxacin than cells expressing wild-type craA (Figure 3.26), indicating the importance of E338.

Several CraA variants (L163A, L246A, Y268A, and L279A) show hyperactive efflux activities in the norfloxacin accumulation assays (Figure 3.27). It is postulated that the alaninesubstitution of L163, L246, and L279 enlarge the binding pocket, while alanine-substitution of Y268 (proximal to cytoplasm) causes an enlarged entrance.

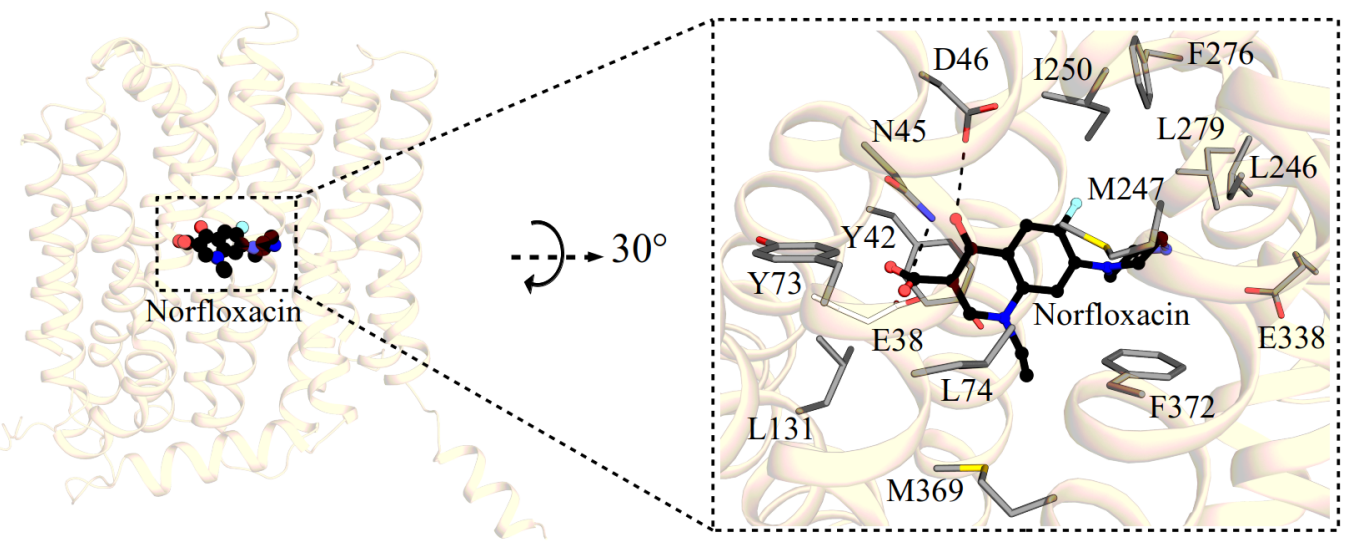

Figure 3.35 In silico analysis of norfloxacin binding to CraA. The CraA model was obtained based on the MdfAQ131A_L339A variant (PDB: 6EUQ) (Zomot et al., 2018). Molecular docking was performed using AutoDock Vina (Trott and Olson, 2010). Inlet shows the docking solution for norfloxacin and CraA model, including the CraA side chain interactions. Putative hydrogen bonds are indicated with dashed lines (Oxygen=red, nitrogen=blue, sulfur=yellow, fluorine=cyan).

In conclusion, docking experiments conducted using AutoDock Vina based on CraA model have predicted the interactions of three CraA substrates in the putative binding pocket. The substrate interactions in the putative binding pocket of CraA are not much deviated from the chloramphenicol binding to MdfA of E. coli. The bindings of chloramphenicol and norfloxacin to CraA are relatively similar, while the binding of lipophilic cation ethidium is slightly different. The functional relevance of the protein-ligand interactions shown in the docking 
solutions was validated through drug susceptibility assays, ethidium transport assays, and norfloxacin accumulation assays carried out using various alanine-substitution variants of CraA (as indicated in section 3.3.2, 3.3.4 and 3.3.5).

\subsection{TetA of $A$. baumannii strain AYE and tigecycline resistance}

\subsubsection{Drug susceptibility assays in E. coli}

To investigate the transport resistance determinant of clinically important tetracyclines, three ORFs on the $A$. baumannii strain AYE genome encoding tetracycline transporters were cloned into PTTQ18. Drug susceptibility assays through MIC determination and drug agar plate assays were performed in E. coli BW25113 $\Delta$ emrEAmdfA or E. coli BW25113 $\triangle e m r E \Delta m d f A \Delta a c r A B$ as host. $\operatorname{Tet} A(A)$ was excluded from further study as the cell growth was inhibited on the control plate without drug, probably due to the toxicity of the protein overproduction by IPTG induction (Figure 3.36a).

A significant increase of MIC for tetracycline, minocycline, doxycycline and tigecycline (256, 4, 16 and 2 fold, respectively) was observed for tetA-expressing BW25113 $\Delta$ emrEAmdfA cells as compared to the negative control (Table 3.3). Cells expressing tetG also exhibited resistance against these tetracyclines except tigecycline. The results of the drug agar plate assay were in accordance to the MICs. No growth was observed for cells expressing tetG on the plate added with tigecycline (Figure 3.36b,c).

To investigate the role of RND efflux pump AcrAB-TolC, $E$. coli BW25113 $\triangle e m r E \Delta m d f A \Delta a c r A B$ was utilized in the drug susceptibility assay. The result shows 4-fold reduced MIC for tetracycline, doxycycline and tigecycline, and 8-fold reduced MIC for minocycline from BW25113 $\triangle$ emrE $\Delta m d f A \Delta a c r A B$ cells expressing tetA compared to the strain with AcrAB (Table 3.3). The lower concentration of drugs used in the drug agar plate assay with host cells lacking $A c r A B$ further confirms the role of RND efflux pumps in resistance towards tetracyclines (Figure 3.36c). 
Table 3.3 MICs of tetracyclines for E. coli harbouring empty vector, pTTQ18_TetA, or pTTQ18_TetG.

\begin{tabular}{|c|c|c|c|c|}
\hline \multirow[b]{2}{*}{ E. coli } & \multicolumn{4}{|c|}{ MIC $(\mu \mathrm{g} / \mathrm{mL})$} \\
\hline & Tetracycline & Minocycline & Doxycycline & Tigecycline \\
\hline BW25113 $\Delta$ emrE $\Delta m d f A+$ pTTQ18 & 0.5 & 1 & 1 & 0.5 \\
\hline BW25113 $\Delta$ emrEsmdfA + pTTQ18_TetA & 128 & 4 & 16 & 1 \\
\hline BW25113 $\Delta$ emrE $\Delta m d f A+$ pTTQ18_TetG & 128 & 8 & 16 & 0.5 \\
\hline BW25113 $\Delta a c r A B \Delta e m r E \Delta m d f A+$ pTTQ18 & 0.125 & 0.125 & 0.125 & 0.125 \\
\hline BW25113 $\Delta a c r A B \Delta e m r E \Delta m d f A+$ pTTQ18_TetA & 32 & 0.5 & 4 & 0.25 \\
\hline BW25113 $\Delta a c r A B \Delta e m r E \Delta m d f A+$ pTTQ18_TetG & 32 & 0.5 & 4 & 0.125 \\
\hline
\end{tabular}

\begin{tabular}{|c|c|}
\hline & Control \\
\hline pTTQ18 & 000000 \\
\hline pTTQ18_TetA & \\
\hline pTTQ18_TetG & 0000 요 \\
\hline TQ18_TetA(A & \\
\hline
\end{tabular}

b.
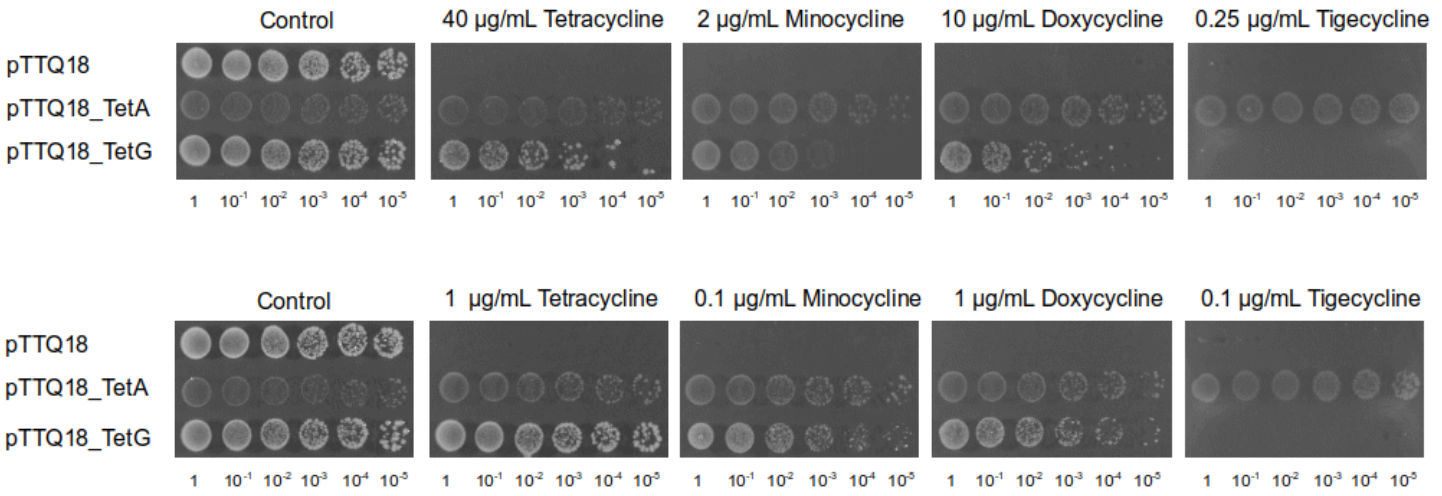

Figure 3.36 Drug susceptibility assay of tetracycline transporters conducted using $(a, b)$ E. coli BW25113 $\triangle$ emrE $\Delta m d f A$ or (c) E. coli BW25113 $\triangle a c r A B \Delta e m r E \Delta m d f A$ as host on LB agar plate supplemented with $0.1 \mathrm{mM}$ IPTG, $50 \mu \mathrm{g} / \mathrm{mL}$ carbenicillin and selected tetracyclines (concentration given above the plate figures). Experiments were conducted at least three times and the results shown are representative. host. (a) The growth of cells overexpressing $\operatorname{tet} A(A)$ was inhibited on a control plate without drug. 


\subsubsection{Drug susceptibility assays in A. baumannii}

The importance of TetA in tigecycline transport in $A$. baumannii was determined by complementation of tetA in $A$. baumannii ATCC 19606. In contrast to $A$. baumannii strain AYE, the type strain ATCC 19606 is susceptible to most antibiotics (Zhu et al., 2020). A. baumannii ATCC 19606 does not contain the TetA studied in this work but possess Tet(C) that shows similarity to $\operatorname{Tet} \mathrm{A}(\mathrm{A})$ of $A$. baumannii strain AYE.

The complementation plasmid plasmid pBAV1K_TetA was introduced into wild-type and the RND gene knockout strains of ATCC 19606 via electroporation ( $\triangle$ adeAB, $\triangle a d e F G$ and $\Delta$ adelJ). The plasmid contains the tetA gene together with the tetR located upstream of tet $A$ of $A$. baumannii strain AYE. Tigecycline susceptibility of the $A$. baumannii strains were tested with drug agar plate assay and via MIC determination.

The MIC measurements indicated that $A$. baumannii WT, $\triangle$ ade $F G$ and $\Delta$ adelJ showed an increase of MIC for tigecycline (from $2 \mu \mathrm{g} / \mathrm{mL}$ to $4 \mu \mathrm{g} / \mathrm{mL}$ ) when pBAV1K_TetA was introduced (Table 3.4). For $A$. baumannii $\triangle a d e A B$, the MIC values were unclear due to the low range of values. In order to confirm the phenotype of the strains, the agar plate assay was additionally conducted. Via this assay, a significant susceptibility of $A$. baumannii $\triangle a d e A B$ strain to tigecycline was observed (no growth on the plate with $0.25 \mu \mathrm{g} / \mathrm{mL}$ tigecycline), indicating that $a d e B$ is the main RND pump involved in tigecycline efflux (Figure 3.37). Knockout of adelJ causes the cells to be susceptible towards tigecycline as well, but to a far lesser extent compared to the $\triangle a d e A B$ strain (Figure 3.37). Surprisingly, $\triangle a d e F G$ strain showed a similar susceptibility phenotype as wild-type $A$. baumannii in the presence of tigecycline, suggesting that adeG is less important in tigecycline efflux in $A$. baumannii (Table 3.4, Figure 3.37). The MIC test also showed that $A$. baumannii strain AYE preexposed with $1 \mu \mathrm{g} / \mathrm{mL}$ tigecycline has significantly higher MIC than the cells that were not exposed to tigecycline, suggesting that there is an overexpression of tetA (Table 3.4). 


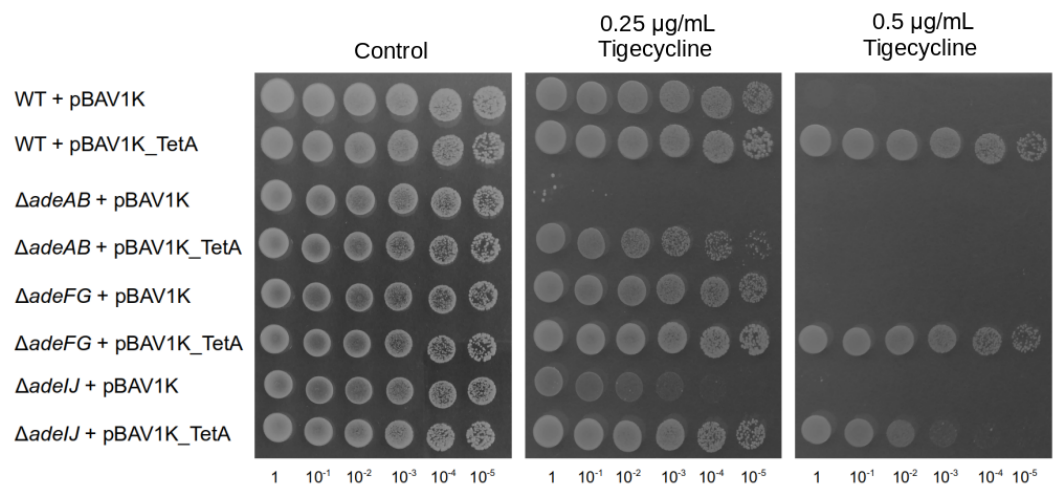

Figure 3.37 Drug susceptibility assay of A. baumannii ATCC 19606 WT and RND-knockout strains complemented with pBAV1K_TetA. Diluted cell cultures (dilution given below the plate figures) were spotted on LB agar plate supplemented with $50 \mu \mathrm{g} / \mathrm{mL}$ kanamycin and tigecycline (concentration given above the plate figures). Experiments were conducted at least three times and the result shown here is representative.

Table 3.4 MIC values of tigecycline for different strains of $A$. baumannii.

\begin{tabular}{|l|c|}
\hline A. baumannii & MIC of tigecycline $(\boldsymbol{\mu g} / \mathbf{m L})$ \\
\hline AYE & 2 \\
\hline AYE preexposed with 1 $\mathrm{gg} / \mathrm{mL}$ tigecycline & $4-8$ \\
\hline ATCC $19606+$ pBAV1K & 2 \\
\hline ATCC $19606+$ pBAV1K_TetA & 4 \\
\hline ATCC $19606 \Delta$ adeAB + pBAV1K & $0.5-1$ \\
\hline ATCC $19606 \Delta$ adeAB + pBAV1K_TetA & 1 \\
\hline ATCC $19606 \Delta$ adeFG + pBAV1K & 2 \\
\hline ATCC $19606 \Delta$ adeFG + pBAV1K_TetA & 4 \\
\hline ATCC $19606 \triangle$ adelJ + pBAV1K & 2 \\
\hline ATCC $19606 \triangle$ adelJ + pBAV1K_TetA & 4 \\
\hline
\end{tabular}

\subsubsection{Differential gene expression analysis of tetA and RND efflux pump genes}

Differential gene expression of tetA gene and RND efflux pump genes (adeB, adeG, and adeJ) in $A$. baumannii strain AYE in the presence of tigecycline was analyzed by RT-qPCR. The result shows that tetA gene was significantly upregulated (tetA: $\Delta \Delta \mathrm{c}_{\mathrm{T}}=4.62 \pm 0.42, \mathrm{p}$ $<0.001$ ) when the cells were exposed to $1 \mu \mathrm{g} / \mathrm{mL}$ of tigecycline for 90 minutes (Figure 3.38). 
The observed mean expressions of $a d e B$ and adeJ were not statistically significantly different in the presence of tigecycline (adeB: $\Delta \Delta \mathrm{CT}_{\mathrm{T}}=-0.54 \pm 0.36, \mathrm{p}=0.19$, adeJ: $\Delta \Delta \mathrm{cT}=-$ $0.44 \pm 0.55, p=0.32$ ). Another RND gene, adeG, was upregulated (adeG: $\Delta \Delta \mathrm{c}_{\mathrm{T}}=2.37 \pm$ $0.57, p=0.001$ ) after tigecycline exposure. However, the tigecycline susceptibility assay has confirmed that the adeG knockout strains did not show susceptibility to tigecycline (Figure 3.38). This indicates that ade $G$ plays a lesser role in tigecycline transport in comparison to ade $B$ and adeJ, probably due to the low amount of transcripts (indicated by low $\Delta \mathrm{c}_{T}$ ) even after upregulation (Figure 3.38).

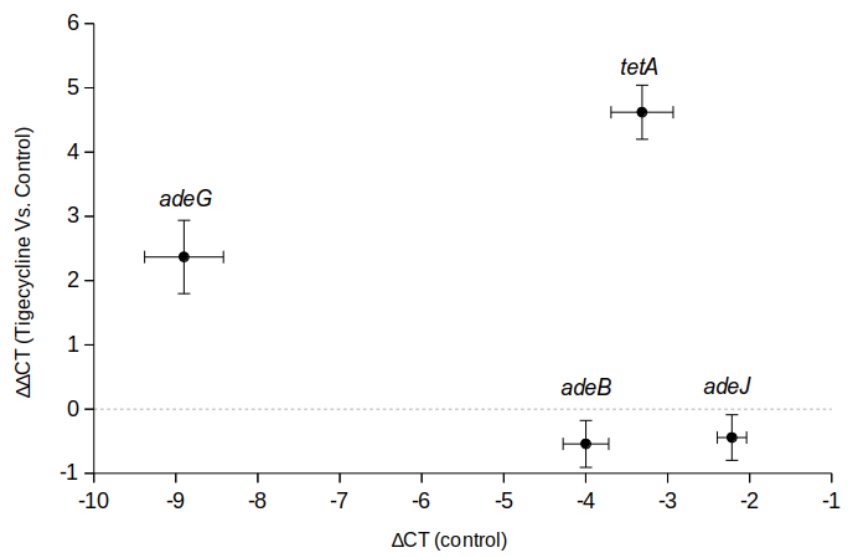

Figure 3.38 Differential gene analysis of tet $A$ and RND efflux pump genes in $A$. baumannii AYE in the absence or presence of tigecycline. Points and error bars represent means and standard errors, respectively, of $n=3-4$ independent experiments. The error bars represent standard errors of mean. The observed mean expressions of ade $B$ and adeJ was not statistically significantly different in the presence of tigecycline (adeB: $\Delta \Delta \mathrm{C}_{\mathrm{T}}=-0.54 \pm$ $0.36, p=0.19$, adeJ: $\Delta \Delta \mathrm{C}_{\mathrm{T}}=-0.44 \pm 0.55, \mathrm{p}=0.32$ ), while tet $A$ and ade $\mathrm{g}$ were significantly induced (adeG: $\Delta \Delta \mathrm{C}_{\mathrm{T}}=2.37 \pm 0.57, \mathrm{p}=0.001$, tet $\left.A: \Delta \Delta \mathrm{C}_{\mathrm{T}}=4.62 \pm 0.42, \mathrm{p}<0.001\right)$ in response to tigecycline presence.

In conclusion, CraA was identified as the major chloramphenicol resistant determinat in $A$. baumannii ATCC 19606. The characterization of CraA through drug susceptibility assays, transport assays in whole cells and proton transport assay confirms that it is a multidrug transporter rather than a specific transporter. Characterization of tetracycline transporters in A. baumannii strain AYE has revealed that TetA is involved in tigecycline efflux activity, together with RND-type efflux pumps. 


\section{CHAPTER 4 : DISCUSSION}

The dissemination of multidrug or even pandrug resistant $A$. baumannii has become a difficult problem in clinical treatment. Various chromosomally encoded efflux systems, both intrinsic and acquired, have been characterized as the main contributors. In this work, the focus is the MFS-type chloramphenicol transporters and tetracycline transporters of the multidrug resistant $A$. baumannii strain AYE. Apart from that, the study of RND tripartite systems of $A$. baumannii (AdeABC, AdeFGH and AdelJK) were included in this work, as it is expected that transporters can form a network to synergistically expel drugs. Since tigecycline is one of the few drugs still effective in the treatment of $A$. baumannii, the effluxmediated tigecycline resistance was investigated. It is possible that the acquisition of known tigecycline transporters, and/or tetracycline transporters in time due to spontaneous mutations will be able to transport tigecycline, leading to resistance against this drug.

\subsection{Chloramphenicol resistance of $A$. baumannii ATCC 19606}

A. baumannii ATCC 19606 was first isolated from the urinary tract of a patient in United States (Hugh and Reese, 1967). It is one of the best characterised strains in this species. In this work, A. baumannii ATCC 19606 knockout strains of RND efflux pumps and MFS-type chloramphenicol transporter CraA were created, followed by differential gene expression analysis of various efflux pumps and outer membrane protein OmpA between the wild-type and knockout strains. In addtion, the wild-type and knockout strains of ATCC 19606 were exposed to subinhibitory concentration of chloramphenicol in order to investigate the response of $A$. baumannii ATCC 19606 under chloramphenicol stress.

Chloramphenicol was isolated by Ehrlich et al. in 1947 from soil bacterium Streptomyces venezuelae. It soon became the first antibiotic to be synthesized chemically in large scale after its relatively simple structure was elucidated (Ehrlich et al., 1947). Its bacteriostatic activity is based on a reversible binding to the peptidyltransferase centre at the $50 \mathrm{~S}$ ribosomal subunit of 70S ribosomes and prevents the binding of tRNA to the A site of the ribosome (Schwarz et al., 2004). Due to potential side effects in neurotoxicity and 
hematological disorders, the drug is generally not recommended but still an alternative for severe life-threatening infections (Dinos et al., 2016).

The major chloramphenicol resistance mechanism of bacteria is the enzymatic inactivation by chloramphenicol acetyltransferases (Murray and Shaw, 1997 ; Schwarz et al., 2004). Bacteria also defense themselves by extruding the drug through efflux pumps of different classes. Chloramphenicol could be exported via specific chloramphenicol transporters such as CmIA found in E. coli, S. enterica, and $P$. aeruginosa, or multidrug transporters such as AcrAB-TolC or MdfA in E. coli (Schwarz et al., 2004 ; Schwarz et al., 2016). It was reported that $E$. coli strains with either acrAB or $m d f A$ overexpression exhibited 4-fold MIC increment for chloramphenicol, while the overexpression of both acrAB and $m d f A$ increased the MIC to 32-fold, emphasizing the interdependent relationship between single- and multi-component efflux pumps (Lee et al., 2000).

Among the clinical $A$. baumannii strains with intrinsic resistance against chloramphenicol, drug efflux or reduced membrane permeability are the main resistance mechanisms, rather than enzymatic inactivation by chloramphenicol acetyltransferases (Vila et al., 1993). Chloramphenicol was reported to be a substrate of the three RND systems from $A$. baumannii BM4587 (Yoon et al., 2015). Moreover, RND efflux systems were assumed to contribute more to intrinsic resistance to chloramphenicol than the MFS pump CraA, based on the observation of MIC reduction from $64 \mu \mathrm{g} / \mathrm{mL}$ to $16 \mu \mathrm{g} / \mathrm{mL}$ for the adeJ knockout strain (Yoon et al., 2015). However, a craA knockout strain was not tested in the study. Interestingly, MIC of chloramphenicol for A. baumannii ATCC 19606 obtained from this work also reduced from $64 \mu \mathrm{g} / \mathrm{mL}$ to $16 \mu \mathrm{g} / \mathrm{mL}$ for adelJ knockout strain, but it further reduced to 2 $\mu \mathrm{g} / \mathrm{mL}$ for $\mathrm{cra} A$ knockout strain (Table 4.1). Furthermore, the upregulation of $c r a A$ observed for wild-type or RND-knockout strains of $A$. baumannii ATCC 19606 upon chloramphenicol exposure confirmed that $\mathrm{CraA}$ is one of the major chloramphenicol resistance determinant in A. baumannii ATCC 19606. Similar observation was also reported previously (Roca et al., 2009).

However, the importance of CraA in chloramphenicol resistance is strongly strain dependent. An inner membrane permease ABUW_0982 was identified as the primary chloramphenicol resistance gene in A. baumannii strain AB5075 (Karalewitz and Miller, 2018). Disruption of 
ABUW_0982 by transposon insertion reduced the MIC of chloramphenicol from $128 \mu \mathrm{g} / \mathrm{mL}$ to $4 \mu \mathrm{g} / \mathrm{mL}$. In contrast, no significant change in MIC was observed when the craA gene was disrupted (128 $\mu \mathrm{g} / \mathrm{mL}$ to $112 \mu \mathrm{g} / \mathrm{mL}$ ) (Karalewitz and Miller, 2018).

Although chloramphenicol was shown to induce the overproduction of CraA in A. baumannii, the regulation of $c r a A$ gene expression remains elusive. A point mutation $12 \mathrm{bp}$ upstream of the craA start codon of $A$. baylyi ADP1 causes an increased stability of the craA mRNA (Brzoska et al., 2013). Two putative promoters of craA gene, the TSS-1 promoter and the TSS-2 promoter discovered through RNAseq are conserved among $A$. baumannii strains (Kröger et al., 2018). Interestingly, these two craA promoters were both downregulated dramatically when cells were changed from rich medium (LB) to nutrient limited medium (M9), subsequently causing low craA expression and chloramphenicol sensitivity to the cells (Kröger et al., 2018).

\subsubsection{Differential gene expression of RND efflux pumps in $A$. baumannii}

Surprisingly, the drastic upregulation of $\operatorname{cra} A$ under chloramphenicol stress was not accompanied by higher expression of RND efflux pumps under the experimental setting in the current study. In fact, the deletion of adelJ or adeAB genes did not lead to significant changes in the expression of the remaining RND systems, with or without chloramphenicol treatment, indicating that the expression of the RND systems is relatively constant in $A$. baumannii ATCC 19606. The adeABC expression of $A$. baumannii strains is under stringent control of the two-component regulatory system adeRS (Marchand et al., 2004). The adelJK system is constitutively expressed in A. baumannii (Damier-Piolle et al., 2008). However, environmental stress is unlikely to select isolates with high adeJ expression, as its overexpression could cause toxicity to the cell (Damier-Piolle et al., 2008, Coyne et al., 2011). A similar observation was reported for $A$. baumannii strain $A Y E$ and $A B 5075$, in which the inactivation of AdeABC and AdelJK pumps did not affect the transcripts of each other and AdeFGH pumps, suggesting a lack of cross-regulation in the expression of these RND pumps (Leus et al., 2020).

The ability to form biofilm contributes to the survival of $A$. baumannii and their dissemination in the hospital environment. $A$. baumannii strains overproducing AdeABC and AdelJK were 
reported to exhibit decreased ability in biofilm formation. In these strains, production of the essential components for biofilm formation i.e. the chaperone-usher-pilus assembly system including CsuA/B and CsuC, the fimbrial protein FimA and the outer membrane protein OmpA are downregulated (Yoon et al., 2015). The overproduction of the RND pumps (ade $A B C$, adelJK, and adeFGH) also results in a significant decrease in the fitness of bacteria (Yoon et al., 2016). A mutant strain of $A$. baylyi ADP1 selected through single exposure of chloramphenicol showed 34 -fold increase in $\operatorname{cra} A(80 \%$ similarity to $\mathrm{cra} A$ of $A$. baumannii) expression. However, the mutant did not exhibit any difference in growth rate compared to the wild-type, while another mutant with 8-fold upregulation of adeJ showed a significant growth defect (Brzoska et al., 2013). We postulate that only craA was drastically upregulated and not other efflux pumps in A. baumannii ATCC 19606, in order to balance the biological cost between resistance and growth under antibiotic stress.

\subsubsection{Outer membrane protein OmpA}

Based on the various reports that emphasized the role of outer membrane proteins in drug resistance of $A$. baumannii (Refer to Chapter 1, section 1.3.3), the gene expression of ompA, which is the most abundant outer membrane protein in $A$. baumannii, was studied in this work. Indeed, higher $\Delta \mathrm{C}_{\mathrm{T}}$ value of ompA compared to the RND genes and craA (Figure $4.7,4.8$ ) indicates higher amount of ompA mRNA transcripts. However, no significant up- or downregulation of ompA was observed, when the cells were grown in the presence of subinhibitory concentration of chloramphenicol. It was also shown in a previous report that overexpression of ompA did not change the MIC value of chloramphenicol (lyer et al., 2018). Therefore, low membrane permeability is unlikely to be the main reason for chloramphenicol resistance.

Due to the limited number of genes that could be studied via RT-qPCR, the presence of other chloramphenicol resistance determinants in A. baumannii ATCC 19606 cannot be ruled out. RNA sequencing is an alternative to obtain a complete profile of the differential gene expression, particularly important to investigate the adaptive mechanisms that enable the survival of craA-knockout $A$. baumannii under chloramphenicol stress. 


\subsection{Chloramphenicol transporters and CraA of A. baumannii strain AYE}

The multidrug-resistant $A$. baumannii strain AYE, which is epidemic in France, is a wellknown strain isolated in 2001 from a patient with pneumonia and a urinary tract infection (Poirel et al., 2003). Whole-genome sequencing of AYE strain revealed a largest resistance island (AbaR1) ever discovered in bacteria (Fournier et al., 2006). The 86,190-bp genomic region which harbours 45 resistance genes is an important contributor to the multidrug resistance phenotype of the AYE strain (Adams et al., 2008).

In this work, five putative chloramphenicol transporters (CraA, CmlA, CmIA5, ABAYE0913, and ABAYE0685) of $A$. baumannii strain AYE were studied (Figure 3.11). CraA shares $45 \%$ and $37 \%$ sequence identity to MdfA and MdtM (Holdsworth and Law, 2012) from E. coli respectively. CmIA5 and $\mathrm{CmIA}$ share $100 \%$ sequence identity with a plasmid-encoded putative chloramphenicol/florfenicol transporter of Salmonella enterica and Klebsiella pneumoniae, respectively. ABAYE0913 and ABAYE0685 share $73 \%$ and $66 \%$ sequence identity with a putative Bcr/CflA family drug resistance efflux transporter of Alkanindiges illinoisensis and Pseudomonadales bacterium, respectively.

The drug susceptibility tests of $E$. coli overexpressing craA have expanded the knowledge on the drug spectrum of this single-component efflux pump, which was reported to be a chloramphenicol specific transporter (Roca et al., 2009). craA-expressing $E$. coli cells confer resistance toward the phenicols (chloramphenicol, thiamphenicol, and florfenicol), monovalent cationic drugs (benzalkonium, $\mathrm{TPP}^{+}$, and ethidium), long dicationic drugs (dequalinium and chlorhexidine), fluoroquinolones (norfloxacin and ciprofoxacin) and anticancer drugs (mitomycin C). Dequalinium and ethidium transport assays conducted in whole cells also indicated that these two drugs are substrates of CraA. Apart from whole cell assays, transport assays using inverted membrane vesicles were conducted to follow the $\mathrm{H}^{+}$/ drug antiport activity of CraA. It further confirmed that dequalinium, TPP ${ }^{+}$, and benzalkonium are substrates of CraA (Refer to Chapter 3, section 3.3).

Dequalinium is an antiseptic agent that contains two quaternary quinolinium units linked by an $\mathrm{N}$-decylene chain (Figure 4.1a). It has multiple mode of actions, primarily based on its ability to disrupt the cell permeability and causes subsequent loss of normal cell enzymatic 
activity (Mendling et al., 2016). The efflux of dicationic drugs by MdfA appears energetically unfavourable, as MdfA exchanges one proton with one monovalent cationic drug molecule (Tirosh et al., 2012 ; Fluman et al., 2014). However, since transport of dequinalinium by MdfA is observed, it was proposed that the transport is possible, provided that the two charged moieties of the divalent cation are separated by a long linker. It is suggested that dequalinium is transported in two consecutive cycles, where each cationic moiety is transported as if it were a separate substrate (Fluman et al., 2014) (Figure 4.1b). The transport of dicationic drugs (dequalinium and chlorhexidine) by CraA is likely to be similar to MdfA. The dicationic drugs with short linkers (methyl viologen, diminazene, and DAPI) were tested in a drug susceptibility assay and the results indicate that these drugs do not belong to the substrates of CraA (Figure S1, Appendix).

a.
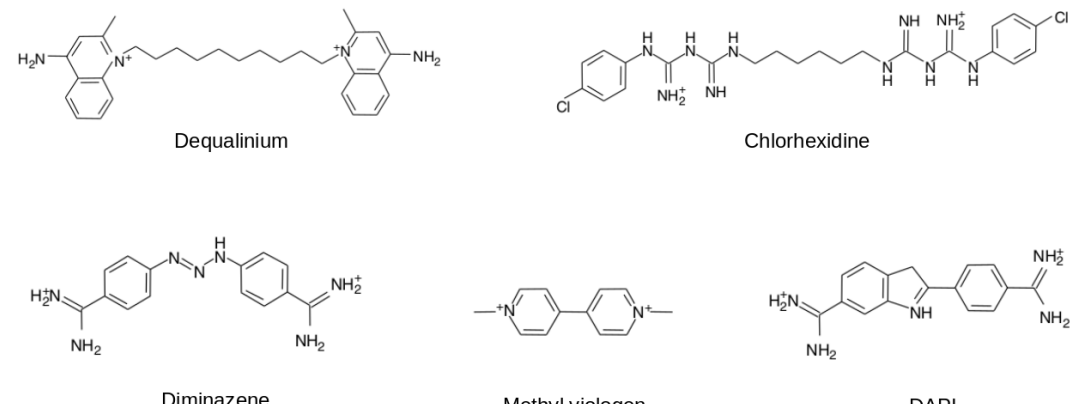

Methyl viologen

DAPI

b.

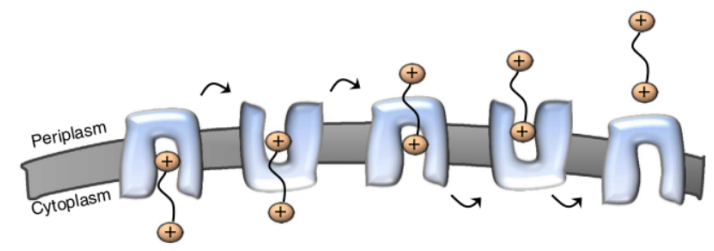

Figure 4.1 The transport mechanism of long linker dicationic compounds by MdfA. (a) Structures of the long linker dicationic (dequalinium and chlorhexidine) and short linker dicationic compounds (diminazene, methyl viologen, and DAPI). (b) MdfA transports long linker dicationic compounds in two successive transport cycles with each of the cationic moiety (sphere) of the substrate is transported in separate transport cycle (adopted from Fluman et al., 2014).

Another substrate of $\mathrm{CraA}$ is mitomycin $\mathrm{C}$ which is commonly used as a chemotherapeutic agent to treat bladder, gastric and pancreatic cancers (Bradner, 2001). It is a potent DNA crosslinker that can block transcription and DNA replication (lyer and Szybalski, 1964). It is used to treat infections of persister A. baumannii, E. coli, Staphylococcus aureus, and Pseudomonas aeruginosa (Kwan et al., 2015). This anticancer drug has been proposed as an alternative drug for the treatment of persistent $A$. baumannii infections (Cruz-Muñiz et al., 
2017). However, as mitomycin $C$ is a substrate of CraA, the treatment should be carried out with extra care, especially when handling with $A$. baumannii strains with craA upregulation.

It was shown through drug susceptibility assays that the fluoroquinolones (norfloxacin and ciprofloxacin) are substrates of CraA (Figure 4.10). The quinolones discovered in the 1960s is the last broad spectrum class of antibacterial agents to enter the market. These bactericidal antibiotics inhibit the enzymes topoisomerase II (DNA gyrase) and topoisomerase IV which maintain the integrity of the supercoiled DNA helix during replication and transcription (Drlica and Zhao, 1997). These compounds bind to the gyrase-DNA complex, forming a ternary complex and subsequently block DNA replication and bacterial growth (Drlica et al., 2012).

Norfloxacin was produced by modification of the prototype quinolone nalidixic acid, while ciprofloxacin emerged from norfloxacin by replacement of the $\mathrm{N}-1$ ethyl with a cyclopropyl group (Figure 4.2). Both drugs have improved activity and pharmacokinetic properties compared to nalidixic acid (Emmerson and Jones, 2003). However, resistance clinical isolates of $A$. baumannii emerged rapidly after the broad usage of fluoroquinolones, mainly caused by alterations of the drug target due to mutations in gyrA and parC (Wisplinghoff et al., 2003). Nevertheless, the role of efflux pumps from the RND superfamily (AdeABC and AdelJK), MATE famiy (AbeM) and MFS (CraA) which could transport fluoroquinolones should not be overlooked (Su et al., 2005 ; Coyne et al., 2011).

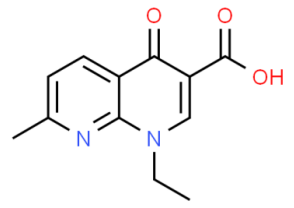

Nalidixic acid

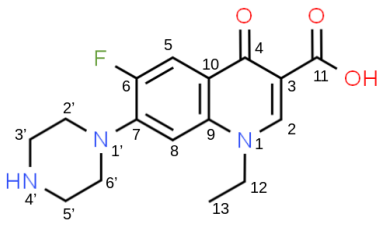

Norfloxacin

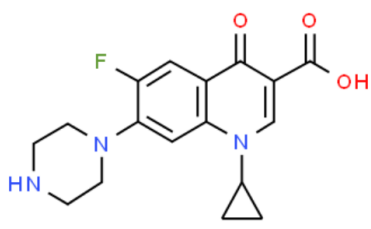

Ciprofloxacin

Figure 4.2 Chemical structures of quinolone antibiotics. 


\subsubsection{Insights into the functionally important residues of CraA}

As CraA was confirmed to be a multidrug efflux pump with a simalr substrate profile as its $E$. coli homolog MdfA, it is postulated that both transporters possess similar proton translocation and drug transport mechanisms. Substitution variants of CraA were created and a functional comparison was made between the CraA and already known MdfA variants.

There are three proton-titratable residues E38 (homologous to E26 of MdfA), D46 (homologous to D34 of MdfA), and E338 (correspond to I327 in MdfA) located in the putative substrate binding pocket of CraA. In E. coli MdfA, E26 and D34 are both essential for active transport of cationic substrates, while for neutral substrates, the carboxylates can compensate for one another (Adler et al., 2004 ; Sigal et al., 2009, 2006). A similar phenomenon was observed in CraA, where E38Q conferred against neutral substrates (chloramphenicol, florfenicol and thiamphenicol), but not against the cationic drug ethidium.

In contrast to E38A, which is totally inactive, alanine-substitution of D46 has a less deleterious effect. D46A is slightly inactive to chloramphenicol and thiamphenicol, but still active to florfenicol, norfloxacin, and benzalkonium. D46A also shows intermediate activity in the ethidium efflux assay. This suggests that D46 is less essential in proton transfer, as compared to MdfA where D34 is an important protonation or deprotonation site (Fluman et al., 2012 ; Yardeni et al., 2017 ; Heng et al., 2015). However, based on the observation that $\mathrm{E} 38 \mathrm{Q}$ is not completely inactive, we postulate that both E38 and D46 can be protonation sites and they are complementing each other.

Another important residue is R112 of MdfA (homologous to R124 of CraA), which is the most evolutionarily conserved residue in MdfA orthologs (Figure 4.11). A positive charge at this position was confirmed to be essential for the function of MdfA (Sigal et al., 2005 ; Heng et al., 2015). R112 is involved in a hydrogen bond network with surrounding residues including G32, N33, T95, Q115, and a water molecule, as well as involved in cation- $\mathrm{T}$ interaction with Y61 and F174 (Figure 4.3) (Heng et al., 2015). Interestingly, R112-Y61 cation-m interaction remains intact but the hydrogen bond network of R112 with the surrounding residues has partially disappeared in the outward open state of MdfA (Figure 4.3) (Nagarathinam et al., 2018). 
MdfA with R112M/Q/E substitutions does not show activity against all substrates, but replacement to $\mathrm{R} 112 \mathrm{H}$ still exhibited efflux activity for most substrates (Sigal et al., 2005 ; Heng et al., 2015). In contrast to the results observed for MdfA, E. coli cells harbouring craA_R124H were completely susceptible to all tested drugs, indicating R124 is essential in CraA and could not be replaced by histadine. Therefore, it is speculated that R124 of CraA is not only involved in a hydrogen bond network with its surrounding residues (G44, N45, C107, and Q127) like postulated for MdfA, but in addition forms a cation- $\pi$ interaction with Y73 (homologous to $Y 61$ of MdfA). In support of this hypothesis, substitution of $Y 73$ to alanine or leucine completely abolished the activity of CraA against all tested drugs. Strikingly, replacement of $Y 73$ to phenylalanine yielded an completely functional CraA. This again is in contrast to the effect of the Y61F substitution in MdfA, that showed only partial activity against chloramphenicol (Heng et al., 2015). We therefore postulate that the R124Y73 cation- $\Pi$ interaction is particularly essential to maintain the integrity of CraA either in its inward open, occluded or outward state.

A

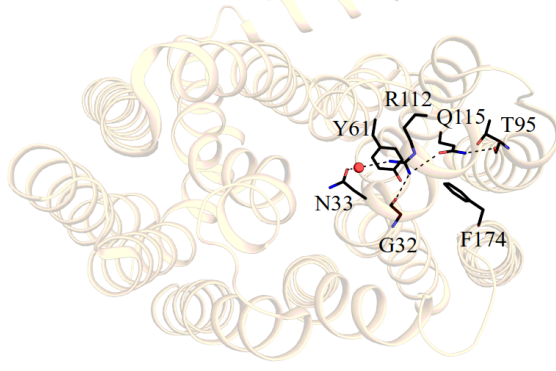

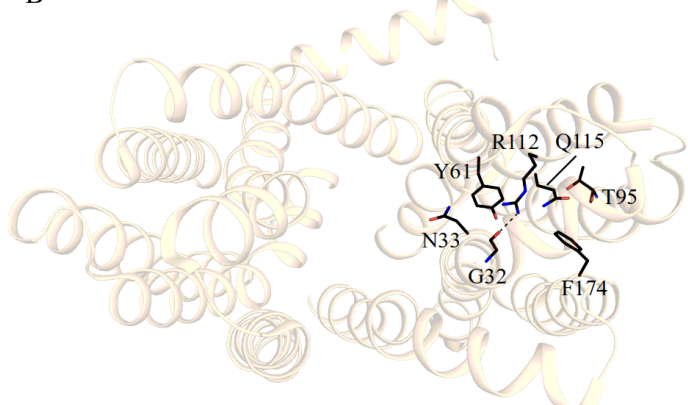

Figure 4.3 Interaction of R112 and Y61 from MdfA. R112 interacts with $\mathrm{Y} 61$ and $\mathrm{F} 174$ by cation- $\mathrm{T}$ interaction in (A) inward open (PDB: 4ZOW) (Heng et al., 2015), (B) outward open (PDB: 6GV1) (Nagarathinam et al., 2018). Red sphere represents water molecule, dotted line represents hydrogen bond.

Alanine substitution of $\mathrm{N} 45$ (homologous to N33 of MdfA) completely abrogated the ability of CraA to confer resistance against all tested drugs. As shown previously, N33 of MdfA is important for chloramphenicol resistance (Heng et al., 2015). The docking solutions support the experimental results, showing that N45 is important in drug binding by forming a hydrogen bond with chloramphenicol, ethidium, norfloxacin (Figure 3.33, 3.34 and 3.35), and possibly all other CraA substrates. Moreover, N33 of MdfA forms a hydrogen bond network with R112 via a water molecule (Figure 4.3). Disruption of such interaction by alaninesubstitution of the N45 might have affect the activity of CraA. 
A previous report suggests that $\mathrm{Y} 30$ of MdfA (homologous to $\mathrm{Y} 42$ of $\mathrm{CraA}$ ) might serve as a mediator in the proton-wire between E26 and D34 (Heng et al., 2015). The increased susceptibility conferred by the Y42A variant against ethidium and norfloxacin (Figure 3.16a, Table 3.2) is supported by its role in the m-stacking interaction as shown in the docking solutions (Figure 3.34 and 3.35). In contrast, the activity of Y42A against chloramphenicol, thiamphenicol, benzalkonium, chlorhexidine, and dequalinium was unaffected (Figure 3.16a, Table 3.2). Therefore, it is predicted that $Y 42$ of $\mathrm{CraA}$ is involved in drug binding rather than having a role in proton-wire.

Surprisingly, alanine-substitution of several residues which are not located in the putative binding pocket also reduced the activity of CraA. Y139, M158, N283, Y346, R347, and K357 are located in proximity to the cleft facing the membrane lipid plane from the cytoplasmic side (Figure 4.4). Substitution of these residues except K357 affect the resistance of $E$. coli against drugs such as norfloxacin, ethidium, florfenicol and/or chlorhexidine. This indicates that some of the membrane-embedded substrates might be taken up from the inner leaflet of the inner membrane rather than from the cytoplasm (Figure 4.4).

It was recently proposed that MdfA may recruit hydrophobic drugs from the inner leaflet of the membrane through a membrane accessible portal between TM5 and TM8, while less hydrophobic drugs may enter from the cytoplasm, between TM4 and TM10 (Yardeni et al., 2020). This further supports the hypothesis that alanine-substitutions of M158 (in TM5), N283 (in TM8), Y139 (in TM4), Y346 and R347 (both in TM10) in CraA have affected the uptake of drugs. 


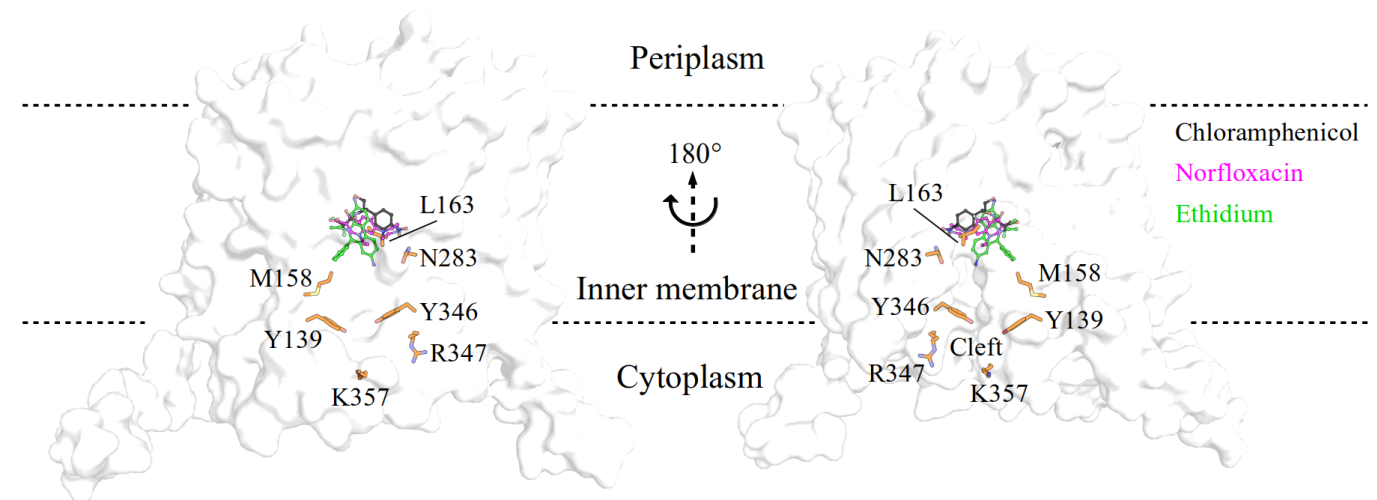

Figure 4.4 Postulated position for residues lining along the shallow cleft of CraA homology model. Docking solutions for chloramphenicol, ethidium and norfloxacin are depicted as ball and sticks model.

In conclusion, the results obtained in this work from various experiments involving 45 CraA substitution variants and docking experiments of three substrates revealed that CraA shows similarities and differences compared to its homolog MdfA. Like MdfA, CraA is postulated to have a drug/proton stoichiometry of $1: 1$. It contains a large drug binding pocket which allows binding of structurally dissimilar substrates, and possibly a flexible protonation site in which the titratable residues E38, D46 and E338 might be able to compensate each other. However, the binding position and chemical interactions between the substrates and the protein could be different between CraA and MdfA. High resolution structures of CraA with bound substrates are essential to unravel and verify the binding and transport mechanisms of CraA predicted in this work.

\subsection{Tetracycline transporters of A. baumannii strain AYE}

Three putative tetracycline transporter genes of $A$. baumannii strain $\mathrm{AYE}, \operatorname{tet} A, \operatorname{tet} G$, and $\operatorname{tet} A(A)$, were heterologously expressed in $E$. coli and drug susceptibility assays were conducted with tigecycline and three other tetracycline derivatives (tetracycline, doxycycline, and minocycline) (Refer to section 1.4.1). The drug susceptibility assays conducted for $E$. coli and $A$. baumannii are drug agar plate dilution assays and MIC measurements via broth dilution method. The MIC determination of tigecycline were conducted with extra precaution to avoid inconsistency and overestimation of the MIC. Tigecycline contains a phenol moiety that is prone to oxidative degradation (Fawzi et al., 2011). Therefore, Freshly prepared 
medium was used within 24 hours, due to the fact that the freshly autoclaved medium contains less amount of dissolved oxygen as suggested by previous studies (Bradford et al., 2005 ; Hope et al., 2005). Apart from that, the tigecycline stock used for every MIC tests was prepared freshly before the experiment, in order to reduce degradation, since tigecycline is known to be relatively unstable after reconstitution and dilution (Fawzi et al., 2011).

Overproduction of TetA or TetG conferred resistance to doxycycline, minocycline and tetracycline in E. coli. Cells expressing tetA conferred also resistance to tigecycline, indicating that TetA could be a resistance determinant for this antibiotic which belongs to the last-resort drug in the treatment of MDR A. baumannii. The importance of TetA in tigecycline transport in $A$. baumannii was further confirmed by complementation of TetA in wild-type $A$. baumannii ATCC 19606 (not encoding tetA on its chromosome) and the RND gene knockout strains $(\triangle a d e A B, \triangle a d e F G$, and $\triangle a d e l J)$.

Gene expression of tet $A$ gene and RND efflux pump genes ade $B$, ade $G$, and adeJ in $A$. baumannii strain AYE in the presence of tigecycline was analysed by quantitative real-time RT-PCR. As expected, tetA gene was significantly upregulated when the cells were exposed to tigecycline. Surprisingly, ade $G$ is also upregulated in the presence of tigecycline. From the tigecycline susceptibility test on $A$. baumannii (Figure 3.37), however, it seems that adeFG plays a minor role in tigecycline efflux. A possible explanation is that the amount of adeG transcripts is very low, compared to $a d e B$ and adeJ, even after upregulation of the gene. However, it cannot be completely ruled out that adeFG also contributes to certain extent in tigecycline resistance. A previous report showed that two strains of $A$. baumannii overexpressing adeFG (ade $A B$ and adelJ were both deleted) exhibited higher MIC for tigecycline as compared to the $\operatorname{ade} A B$ and adelJ double-knockout strain without adeFG overexpression (Coyne et al., 2010).

\subsubsection{Efflux-based tigecycline resistance}

Tigecycline resistance has been attributed to the action of RND-type efflux pumps and is dependent on the regulation by several global transcriptional regulators of RND efflux pumps. Although tigecycline is not recognized by the Tet efflux transporter (TetB, TetC, and TetK), but it is a possible substrate of AcrAB and its close homolog AcrEF in E. coli and 
Salmonella (Hirata et al., 2004 ; Horiyama et al. 2011). Several studies also emphasized the role of transcriptional regulators in tigecycline-nonsusceptible Klebsiella pneumoniae strains. RamR is a negative regulator of $\operatorname{ram} A$ and deletion or loss-of-function mutations of RamR cause significant increase in $\operatorname{ram} A$ expression and lead to upregulation of acrAB (Ruzin et al., 2005 ; Sheng et al., 2014 ; Chiu et al., 2017).

In the context of $A$. baumannii, adeABC was the first system to be proposed as an effluxbased tigecycline resistance mechanism (Peleg et al., 2007). Many other reports also highlighted the overexpression of $a d e A B$ as a prevalent mechanism for the decreased susceptibility to tigecycline (Hornsey et al., 2010 ; Ruzin et al., 2010 ; Rumbo et al., 2013 ; Lin et al., 2017). Another RND system of A. baumannii, AdelJK, also contributes to tigecycline resistance (Damier-Piolle et al. 2008 ; Rumbo et al., 2013 ; Lin et al., 2017) and was found to act in a synergistic fashion with AdeABC to extrude tigecycline (Damier-Piolle et al., 2008). The upregulation of RND efflux pumps is related to the mutations in the genes encoding their local regulators. Disruption of adeS or adeN by insertion sequences (ISs) increase $a d e B$ or adeJ expression, respectively, and lead to an increased MIC for tigecycline (Gerson et al., 2018). Single amino acid substitutions in AdeR and AdeS are frequently reported to be crucial in reducing tigecycline susceptibility of $A$. baumannii (Hornsey et al., 2011 ; Montaña et al., 2015 ; Sun et al., 2016 ; Hornsey and Wareham, 2018). Several different mutations observed in adeN also lead to an increased MIC for tigecycline (Gerson et al., 2018).

Despite all these studies, the exact tigecycline resistance mechanisms in $A$. baumannii remains puzzling. Yoon et al. 2013 showed that in clinical tigecycline-resistant strains, expression of ade $A B C$ was not correlated with tigecycline MIC and could be due to another determinant. Another study also found that the RND efflux pumps (AdeABC, AdeFGH, and AdelJK) were not overexpressed in A. baumannii ATCC 19606 strains selected through serial exposure to tigecycline, however a truncated version of an S-adenosyl-L-methionine (SAM)-dependent methyltransferase appeared to contribute to the decreased tigecycline susceptibility (Chen et al. 2014). 


\subsubsection{Cooperation between single-component efflux pumps and RND tripartite systems}

A previous study proposed that AcrAB-TolC is essential for the acquisition of tetracycline resistance genes by horizontal gene transfer, as the acquisition of a plasmid comprising the tetA gene is not followed by the production of the encoded TetA protein in AcrAB-TolC deletion strain, resulting in tetracycline susceptibility (Nolivos et al., 2019). They proposed that the AcrAB-TolC system plays a crucial role by reducing the concentration of tetracycline in the cell to a level that that the protein synthesis of TetA from the newly acquired tetAcontaining plasmids can proceed. The continuation of protein synthesis in the presence of tetracycline allows cells to produce more TetA proteins to initiate efflux and subsequently tetracycline resistance is established (Figure 4.5).

(i)

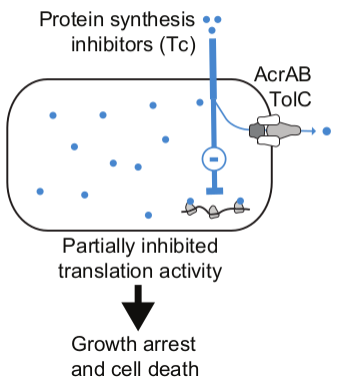

(ii)

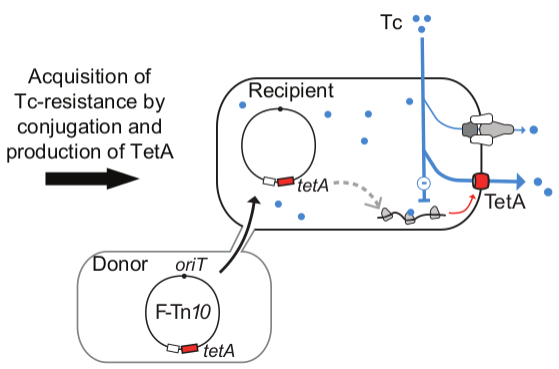

(iii)

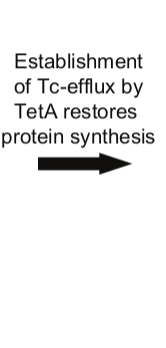

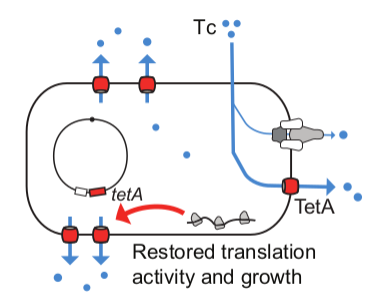

Figure 4.5 Summary of the model for the role of AcrAB-TolC in the acquisition of resistance by conjugation. (i) Tetracycline (Tc) enters the cell and inhibit protein synthesis. At inhibitory concentrations, AcrAB-TolC efflux activity is not sufficient to support cell growth. (ii) If the cell acquires Tc-resistance genes by conjugation, AcrABTolC efflux is required for the production of the TetA protein encoded by the newly acquired plasmid. Production of TetA enhances Tc-efflux, thus facilitating the synthesis of additional TetA. (iii) Further TetA production triggers a positive feedback loop, which eventually allows protein synthesis activity to be restored and effective Tcresistance to be established (adopted from Nolivos et al. 2019).

Although the importance of RND system in the acquisition of resistance determinant is very well examined in the proposed model (Figure 4.5), TetA which is located in the inner membrane of $E$. coli is unlikely to extrude tetracyclines across the outer membrane. So again, the tripartite system AcrAB-TolC plays an important role during the efflux activity, by working cooperatively with TetA, in which TetA exports the drug from the cytoplasm to the periplasm, where the drug is further transported by the RND tripartite system and exported 
to the extracellular environment at high turnover rates (Figure 4.6). The coordination between the RND tripartite system and other single-component efflux pumps is essential for successful efflux-mediated drug resistance (Lee et al., 2000 ; de Cristóba et al. 2006 ; Tal and Schuldiner, 2009).

It is postulated that the immediate response of non-resistant $A$. baumannii when facing tigecycline stress is the up-regulation of the single-component efflux pump TetA, which transports tigecycline from the cytoplasm to the periplasm, from where the drug is further transported across the outer membrane by the constitutively present RND tripartite systems AdeABC and AdelJK. Similar hypothesis also applied to the single-component multidrug efflux pump CraA. Drug susceptibility assays conducted with craA-expressing cells in the $\triangle a c r A B$ background are more susceptible to all tested drugs as compared to cells expressing acrB (Figure 3.13b). It is therefore postulated that CraA and RND-type systems, including AdeABC, AdeFGH and AdelJK, operate in synergy to expel drugs from the cells.

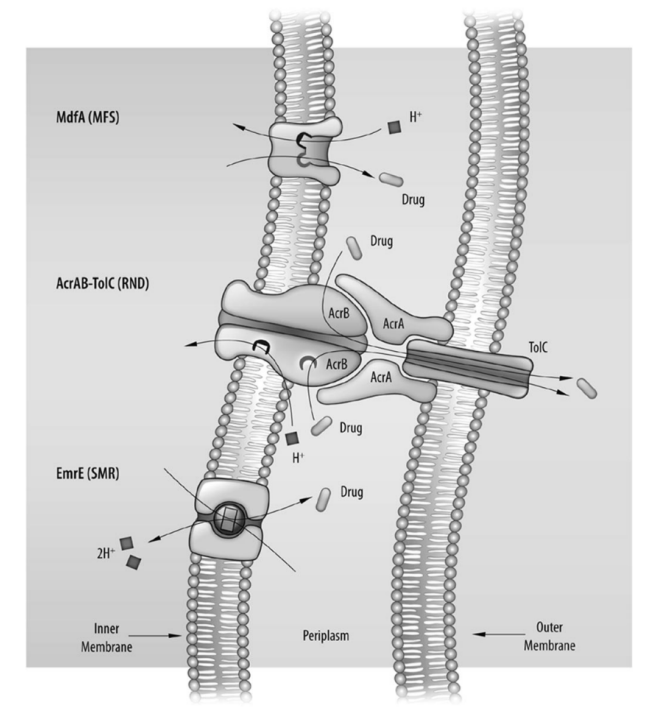

Figure 4.6 Model of interaction between AcrAB-TolC and other efflux pumps in $E$. coli. The single-component transporters, EmrE and MdfA, remove the drug from the cytoplasm into the periplasm, where AcrAB-TolC captures and extrudes the drug out of the cell (adopted from Tal and Schuldiner 2009).

\subsubsection{Mutations in TetA could lead to tigecycline resistance}

The drug tigecycline was developed to cope with the high occurrence of tetracycline resistance caused by a wide range of MFS-type tetracycline transporters (Hirata et al., 2004 ; Pankey et al., 2005). However, there is an evidence that the expression of tetA gene can decrease the sensitivity of tigecycline in Salmonella spp. (Akiyama et al., 2013). 
Interestingly, sequence alignment shows that tetA from Salmonella carries a double frameshift mutation in codons 201, 202 and 203 as compared to the tet $A$ from plasmid RP1 (accession no. X00006), causing amino acid substitutions S201A, F202S, and V203F. Surprisingly, this mutated tet $A$ gene is more commonly detected as indicated by deposition of this variant to the GenBank in comparison to the RP1-linked tetA variant, including the tet $A$ of $A$. baumannii strain AYE. These codons are located at the largest cytoplasmic loop between transmembrane helices 6 and 7 (Figure 4.7) and they are postulated to impact the selective gating of tetracyclines (Tuckman et al., 2000). The changes in codons 201-203 resulting in slightly less bulky residues at these positions, which may allow the entrance of minocycline, tigecycline, and eravacycline (TP-434) (Tuckman et al., 2000 ; Hentschke et al., 2010 ; Grossman et al., 2012).

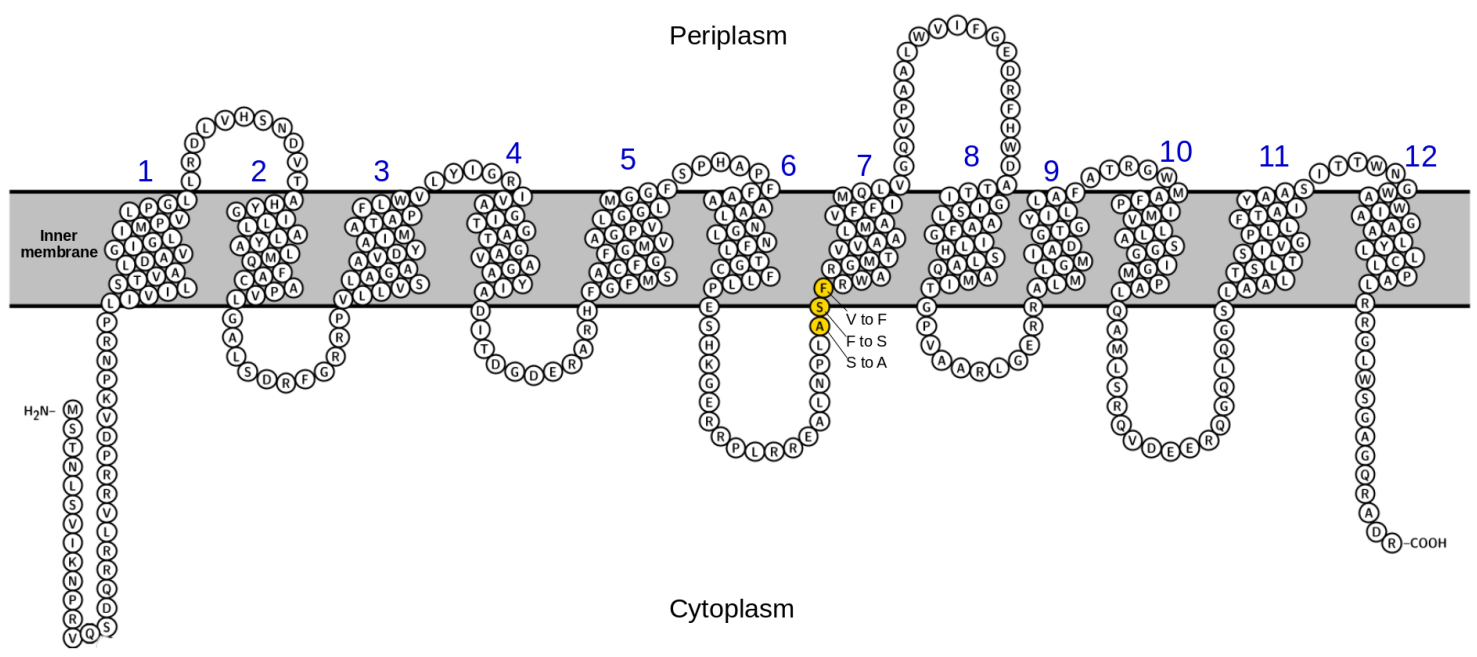

Figure 4.7 Topology model of TetA. The substitutions of the three residues S201A, F202S and V203F are indicated in yellow. The transmembrane domains are labeled with numbers. The model was created using the programme 'Protter' (http://wlab.ethz.ch/protter) by providing UniProt Protein accesion number Q2FCZ4_ACIBA.

Alarmingly, one study showed that a single mutation in tetA can lead to increased MIC of tigecycline as compared to the non-mutated tetA if overexpressed in $E$. coli (Linkevicius et al. 2016). The latter study describes the libraries creation of mutagenized tet $A$ sequences through error-prone PCR and resulted in the identification of several mutations (S251A and G300E) that led to higher tigecycline MIC (Linkevicius et al. 2016). The amino acid substitution S251A was also identified in TetA from a carbapenem-resistant Klebsiella 
pneumoniae (CRKP) clinical isolate (Du et al., 2018). Moreover, tigecycline-resistant isolates with MIC values of $32 \mathrm{mg} / \mathrm{L}$ taken from the patient during the tigecycline treatment revealed the substitution S251A in TetA (Du et al., 2018). The patient eventually passed away, post us a warning that tetA mutation under tigecycline selective pressure could lead to treatment failure (Du et al., 2018).

In conclusion, TetA located on the Tn1721-like transposon is associated with conjugative and transmissible plasmids, posing the threat of horizontal gene transfer between resistant and susceptible strains (Frech and Schwarz, 1999 ; Ribera et al., 2003). A transcriptomic analysis of multidrug-resistant $A$. baumannii MDR-ZJ06 revealed that some MFS transporters involved in the stress response and drug resistance were upregulated, while beta-lactamases, such as OXA-23 and $\mathrm{AmpC}$, were downregulated in the presence of subinhibitory concentration of tigecycline (Hua et al., 2014). Tigecycline was also identified as one of the strongest inducer of efflux pumps gene expression in A. baumannii (Lin et al., 2017). Therefore, the administration of tigecycline has to be carried out with extra precaution and the spread of tet $A$ should be monitored closely in clinical isolates. 


\section{APPENDIX}

\subsection{Methods}

\subsubsection{Cloning via Gibson assembly}

Gibson assembly depends on the joining of complimentary overhang fragments through an isothermal reaction $\left(50^{\circ} \mathrm{C}\right)$ involving three enzymes: T5 exonuclease, DNA polymerase and T4 DNA ligase. The primers for amplification of vector backbone and DNA insert contain additional overlap sequence of 20 to 30 bp homology to insert or vector. PCR was conducted using ingredients described in Table S1, with the PCR programme shown in Table S2.

Table S1 PCR ingredients for amplification of backbone vectors and DNA insert.

\begin{tabular}{|l|c|}
\hline Chemicals & Volume $(\boldsymbol{\mu L})$ \\
\hline $5 \times \mathrm{GC}$ buffer & 10.0 \\
\hline Forward primer (Stock: $10 \mathrm{p} \mathrm{mol})$ & 1.2 \\
\hline Reverse primer (Stock: $10 \mathrm{p} \mathrm{mol})$ & 1.2 \\
\hline DNA (Stock: $100 \mathrm{ng} / \mathrm{\mu l})$ & 0.5 \\
\hline dNTP $(10 \mathrm{mM})$ & 1.5 \\
\hline dd $_{2} \mathrm{O}$ & 34.6 \\
\hline Phusion high-fidelity DNA polymerase & 1.0 \\
\hline Total volume & 50.0 \\
\hline
\end{tabular}

Table S2 PCR programme for amplification of backbone vectors and DNA insert.

\begin{tabular}{|c|c|c|c|}
\hline Steps & Temperature & Time & Cycle \\
\hline Initial denaturation & $98^{\circ} \mathrm{C}$ & 30 seconds & 1 \\
\hline Denaturation & $98^{\circ} \mathrm{C}$ & 10 seconds & \multirow{2}{*}{30} \\
\hline Annealing & $58^{\circ} \mathrm{C}$ & 15 seconds & \\
\hline Extension & $72^{\circ} \mathrm{C}$ & 30 s $/ 1000 \mathrm{bp}$ & \\
\hline Final extension & $72^{\circ} \mathrm{C}$ & 10 minutes & 1 \\
\hline
\end{tabular}


Both PCR products (vector backbone and insert) were subjected to agarose gel electrophoresis ( $1 \%$ agarose gel). The samples and $1 \mathrm{~kb}$ DNA marker were loaded to the gel and electrophoresis was carried out at $120 \mathrm{~V}$ for 60 minutes. Digestion was done to remove the template plasmid by mixing $1 \mu \mathrm{L}$ of Dpnl, $45 \mu \mathrm{L}$ of PCR product (vector backbone) and 5 $\mu \mathrm{L}$ of $10 \mathrm{x}$ FD buffer. The mixture was incubated at $37^{\circ} \mathrm{C}$ for 1 hour $30 \mathrm{~min}$. Both PCR products were purified with DNA Clean \& Concentrator-5 kit (Zymo Research). For Gibson assembly reaction, $5 \mu \mathrm{L}$ of the purified PCR products (80 $\mathrm{ng}$ vector backbone and $80 \mathrm{ng}$ insert) was mixed with $15 \mu \mathrm{L}$ of assembly mixture. The assembly mixture was prepared according to ingredients shown in Table S3 and Table S4. The reaction mixture was incubated at $50^{\circ} \mathrm{C}$ for 1 hour, followed by transformation of $E$. coli MACHT1 with $2 \mu \mathrm{L}$ of the reaction mixture.

Table S3 Composition of assembly mixture.

\begin{tabular}{|l|c|}
\hline Chemicals & Volume $(\mu \mathrm{L})$ \\
\hline $5 x$ ISO buffer (Refer to Table S4) & 80.0 \\
\hline $10 \mathrm{U} / \mu \mathrm{l}$ T5 exonuclease & 0.16 \\
\hline $2 \mathrm{U} / \mu \mathrm{l}$ Phusion polymerase & 5.0 \\
\hline $40 \mathrm{U} / \mu \mathrm{l}$ Taq DNA ligase & 40.0 \\
\hline $\mathrm{ddH}_{2} \mathrm{O}$ & 174.84 \\
\hline Total volume & 300.0 (aliquoted and kept at $\left.-21^{\circ} \mathrm{C}\right)$ \\
\hline
\end{tabular}

Table S4 Composition of 5x ISO buffer (6 mL).

\begin{tabular}{|l|l|}
\hline Chemicals & Volume / Mass \\
\hline $1 \mathrm{M} \mathrm{Tris/HCl}, \mathrm{pH} 7.5$ & $3 \mathrm{~mL}$ \\
\hline $1 \mathrm{M} \mathrm{MgCl}_{2}$ & $300 \mu \mathrm{L}$ \\
\hline $100 \mathrm{mM}$ dGTP & $60 \mu \mathrm{L}$ \\
\hline $100 \mathrm{mM}$ dATP & $60 \mu \mathrm{L}$ \\
\hline $100 \mathrm{mM}$ dCTP & $60 \mu \mathrm{L}$ \\
\hline $100 \mathrm{mM}$ dTTP & $60 \mu \mathrm{L}$ \\
\hline $1 \mathrm{M} \mathrm{DTT}$ & $300 \mu \mathrm{L}$ \\
\hline PEG8000 & $1.5 \mathrm{~g}$ \\
\hline $100 \mathrm{mM} \mathrm{NAD}$ & $600 \mu \mathrm{L}$ \\
\hline
\end{tabular}




\subsubsection{SDS-PAGE and Western blot}

Sample mixed with Sample Buffer (Table S6) was loaded onto the polyacrylamide gel prepared according to ingredients shown in Table S5. Protein marker (PageRuler Prestained Protein Ladder) of $5 \mu \mathrm{L}$ was also loaded. The electrophoresis was carried out at $40 \mathrm{~mA}$ in SDS Running Buffer (Table S7) for 45 minutes.

Table S5 Composition of separating gel and stacking gel.

\begin{tabular}{|l|c|c|}
\hline Chemicals & $\begin{array}{c}\mathbf{1 2 . 5 \%} \\
\text { separating gel }\end{array}$ & $\begin{array}{c}\mathbf{5 \%} \\
\text { stacking gel }\end{array}$ \\
\hline $30 \%$ bis-acrylamide & $3.8 \mathrm{~mL}$ & $0.5 \mathrm{~mL}$ \\
\hline $1.88 \mathrm{M}$ Tris/HCl, pH 8.8 & $1.8 \mathrm{~mL}$ & - \\
\hline $0.635 \mathrm{M}$ Tris/HCl, $\mathrm{pH} 6.8$ & - & $0.6 \mathrm{~mL}$ \\
\hline $0.5 \%(w / v)$ SDS & $1.8 \mathrm{~mL}$ & $0.6 \mathrm{~mL}$ \\
\hline $\mathrm{H}_{2} \mathrm{O}$ & $2.4 \mathrm{~mL}$ & $1.5 \mathrm{~mL}$ \\
\hline Tetramethylethylenediamine (TEMED) & $8.0 \mu \mathrm{L}$ & $3.0 \mu \mathrm{L}$ \\
\hline $10 \%(w / v)$ ammonium persulfate (APS) & $45.0 \mu \mathrm{L}$ & $15.0 \mu \mathrm{L}$ \\
\hline
\end{tabular}

Table S6 Composition of Sample Buffer.

\begin{tabular}{|l|l|}
\hline \multicolumn{2}{|l|}{ 5x Non-reducing Sample Buffer (loading dye) } \\
\hline Chemicals & Concentration \\
\hline Tris/HCl, pH 6.8 & $500 \mathrm{mM}$ \\
SDS & $8 \%(\mathrm{w} / \mathrm{v})$ \\
Glycerol & $40 \%(\mathrm{v} / \mathrm{v})$ \\
Bromophenol Blue & $0.004 \%(\mathrm{w} / \mathrm{v})$ \\
\hline
\end{tabular}

Table S7 Composition of SDS Running Buffer.

\begin{tabular}{|l|l|}
\hline Chemical & Concentration \\
\hline Tris $/ \mathrm{HCl}$ & $25 \mathrm{mM}$ \\
Glycine & $192 \mathrm{mM}$ \\
SDS & $0.1 \%(\mathrm{w} / \mathrm{v})$ \\
\hline
\end{tabular}


The gel, filter papers and nitrocellulose membrane were soaked with Transfer Buffer (Table S8) for 5 minutes. Electrotransfer was carried out at $10 \mathrm{~mA}$ for 25 minutes (Trans-Blot Turbo Transfer System, Bio-Rad). For blocking step, 3\% BSA in Tris-buffered saline Tween (TBST) buffer (Table S8) was added to the membrane and incubated for one hour at room temperature or overnight at $4^{\circ} \mathrm{C}$. It is followed by washing with TBST buffer for 5 minutes on a shaker. This step was done for three times. The first antibody (1:2000 6x anti-histidine in TBST $+1 \%$ BSA) was added and incubated for one hour on a shaker, followed by three time washing with TBST buffer. The second antibody (1:1500 anti-mouse antibody in TBST+1\%BSA) was added and the same proceduce was repeated. The membrane was equilibrated with alkaline phosphatase (AP) buffer (Table S8) for five minutes. For the development, the membrane was incubated with $20 \mathrm{~mL}$ of AP buffer added with $66 \mu \mathrm{L}$ of $5 \%$ 5-bromo-4-chloro-3-indolyl phosphate (BCIP) and $33 \mu \mathrm{L}$ of $5 \%$ nitroblue tetrazolium (NBT) until a band was observed. The reaction was stopped with 50 mM EDTA.

Table S8 Buffers used in Western blot.

\begin{tabular}{|c|c|c|c|c|c|}
\hline \multicolumn{2}{|c|}{ Transfer Buffer } & \multicolumn{2}{|c|}{ TBST buffer } & \multicolumn{2}{|c|}{ AP buffer } \\
\hline Chemical & Concentration & Chemical & Concentration & Chemical & Concentration \\
\hline $\begin{array}{l}\text { Tris/HCl } \\
\text { Glycine } \\
\text { SDS } \\
\text { Methanol }\end{array}$ & $\begin{array}{l}25 \mathrm{mM} \\
192 \mathrm{mM} \\
0.1 \%(\mathrm{v} / \mathrm{v}) \\
20 \%(\mathrm{v} / \mathrm{v})\end{array}$ & $\begin{array}{l}\text { Tris/HCl } \\
(\mathrm{pH} 8) \\
\mathrm{NaCl} \\
\text { Tween } 20\end{array}$ & $\begin{array}{l}10 \mathrm{mM} \\
150 \mathrm{mM} \\
0.05 \%\end{array}$ & $\begin{array}{l}\text { Tris/HCl } \\
(\mathrm{pH} 9.5) \\
\mathrm{NaCl} \\
\mathrm{MgCl}_{2}\end{array}$ & $\begin{array}{l}100 \mathrm{mM} \\
100 \mathrm{mM} \\
5 \mathrm{mM}\end{array}$ \\
\hline
\end{tabular}

\subsubsection{Preparation of chemically competent $E$. coli cells}

Overnight culture of $300 \mu \mathrm{L}$ was inoculated into $100 \mathrm{~mL}$ of LB liquid medium and incubated at $37^{\circ} \mathrm{C}, 180 \mathrm{rpm}$ until $\mathrm{OD}_{600}$ reached around 0.6 . The cells were harvested by centrifugation at $3500 \mathrm{~g}$ for 10 minutes and resuspended in $20 \mathrm{~mL}$ of ice-cold sterile $60 \mathrm{mM} \mathrm{CaCl}$ supplemented with $15 \%$ glycerol. After one hour of incubation on ice, the cells were centrifuged at $3500 \mathrm{~g}$ for 10 minutes and resuspended in $2 \mathrm{~mL}$ of ice-cold sterile $60 \mathrm{mM}$ $\mathrm{CaCl}_{2}$ supplemented with $15 \%$ glycerol, followed by incubation on ice for one hour. The cells were aliquoted $(50 \mu \mathrm{L})$, flash-cooled with liquid nitrogen and kept at $-80^{\circ} \mathrm{C}$. 


\subsubsection{Transformation via heat-shock method}

The competent cells added with $0.5 \mu \mathrm{L}$ plasmid or $2 \mu \mathrm{L}$ reaction mixture were incubated on ice for 20 minutes, heat shock at $42^{\circ} \mathrm{C}$ for 60 seconds and cooled down on ice for 5 minutes. The cells were grown in $200 \mu \mathrm{L}$ of LB medium for 45 minutes at $37^{\circ} \mathrm{C}$ with $900 \mathrm{rpm}$. The transformants were then plated on LB agar supplemented with antibiotics and incubated overnight at $37^{\circ} \mathrm{C}$.

\subsubsection{Plasmid minipreparation}

For isolation of the plasmid, a single colony was inoculated into $5 \mathrm{~mL}$ of LB medium supplemented with antibiotics ( $50 \mu \mathrm{g} / \mathrm{mL}$ kanamycin or carbenicillin). The cells were grown overnight at $37^{\circ} \mathrm{C}$ overnight, $180 \mathrm{rpm}$. Plasmid minipreparation was performed using Zyppy Plasmid Miniprep Kit (Zymo Research). The concentration of DNA was measured at 260/280 $\mathrm{nm}$ with a Tecan microplate reader.

\subsubsection{Preparation of electrocompetent A. baumannii ATCC 19606 cells}

Overnight culture of $A$. baumannii ATCC19606 was inoculated to $200 \mathrm{~mL}$ of LB medium and grown till $\mathrm{OD}_{600}$ of around 0.5 . Cells were harvested with centrifugation at $4000 \mathrm{rpm}$ for 10 minutes. The cell pellet was washed with ice-cold sterile $\mathrm{ddH}_{2} \mathrm{O}$ through centrifugation and resuspension for four times to remove salts and other impurities, followed by washing with ice-cold sterile $10 \%$ glycerol for one time. The cells were resuspended in $250 \mu \mathrm{L}$ of $10 \%$ glycerol, aliquoted $(50 \mu \mathrm{L})$ and flashed-cool with liquid nitrogen.

\subsubsection{Transformation via electroporation}

The plasmid (pBIISK_sacB/kanR or pBAV1K) was transformed into electrocompetent $A$. baumannii ATCC 19606 cells by electroporation at $2.5 \mathrm{kV}, 200 \Omega$ and $25 \mu \mathrm{F}$ in a pre-cooled electroporation cuvette. One $\mathrm{mL}$ of pre-warmed $\mathrm{SOC}$ medium $\left(37^{\circ} \mathrm{C}\right)$ was immediately added to the cell suspension. Cells were incubated at $37^{\circ} \mathrm{C}$ for 1 hour, $130 \mathrm{rpm}$. The 
transformants were plated onto LB agar supplemented with $50 \mu \mathrm{g} / \mathrm{mL}$ kanamycin and incubated overnight at $37^{\circ} \mathrm{C}$.

\subsection{Results}

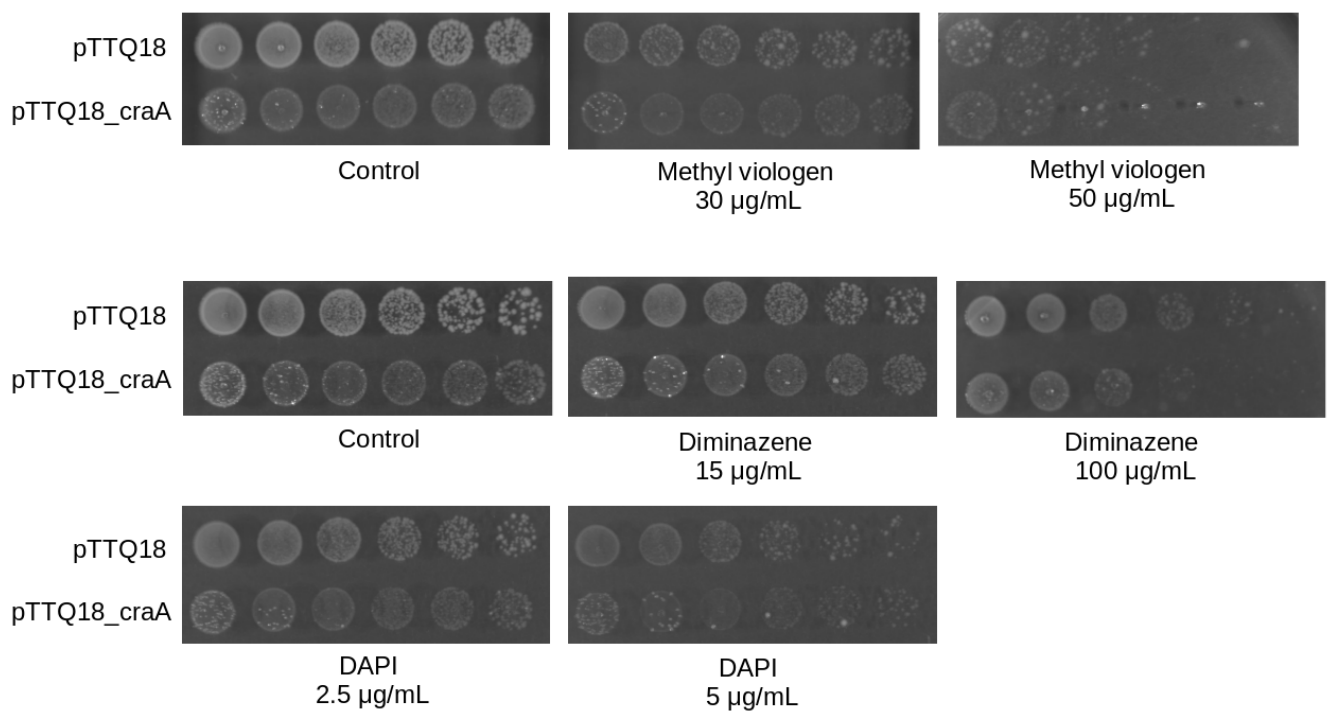

Figure S1 Drug susceptibility assays of $E$. coli BW25113 $\Delta$ emrE $\Delta m d f A$ harbouring $c r a A$ of $A$. baumannii strain AYE. The assays were conducted using LB agar plates supplemented with $0.2 \mathrm{mM} \mathrm{IPTG}, 100 \mu \mathrm{g} / \mathrm{mL}$ ampicillin and the indicated short linker dicationic drugs (drugs and concentrations given below the plate figures). Experiments were conducted at least three times and the results shown here are representative. 


\section{REFERENCES}

Adams MD, Goglin K, Molyneaux N, Hujer KM, Lavender H, Jamison JJ, MacDonald IJ, Martin KM, Russo T, Campagnari AA, Hujer AM, Bonomo RA, Gill SR (2008) Comparative genome sequence analysis of multidrug-resistant Acinetobacter baumannii. J Bacteriol. 190(24):8053-64. doi: 10.1128/JB.00834-08.

Adler J, Lewinson O, Bibi E (2004) Role of a conserved membrane-embedded acidic residue in the multidrug transporter MdfA. Biochemistry 43(2):518-25. doi: 10.1021/bi035485t.

Agerso $Y$, Guardabassi L (2005) Identification of Tet 39, a novel class of tetracycline resistance determinant in Acinetobacter spp. of environmental and clinical origin. J Antimicrob Chemother 55:566-569. doi: 10.1093/jac/dki051.

Agerso Y, Petersen A (2007) The tetracycline resistance determinant Tet 39 and the sulphon-amide resistance gene sulll are common among resistant Acinetobacter spp. isolated from integrated fish farms in Thailand. J Antimicrob Chemother. 59:23-27. doi: 10.1093/jac/dkl419.

Akers KS, Mende K, Yun HC, Hospenthal DR, Beckius ML, Yu X, Murray CK (2009) Tetracycline susceptibility testing and resistance genes in isolates of Acinetobacter baumannii-Acinetobacter calcoaceticus complex from a U.S. military hospital. Antimicrob Agents Chemother. 53(6):2693-5. doi: 10.1128/AAC.01405-08.

Akiyama T, Presedo J, Khan AA (2013) The tetA gene decreases tigecycline sensitivity of Salmonella enterica isolates. Int J Antimicrob Agents 42:133-140. doi: 10.1016/j.jjantimicag.2013.04.017.

Amin IM, Richmond GE, Sen P, Koh TH, Piddock LJ, Chua KL (2013) A method for generating marker-less gene deletions in multidrug-resistant Acinetobacter baumannii. BMC Microbiol. 13:158. doi: 10.1186/1471-2180-13-158.

Bergeron J, Ammirati M, Danley D, James L, Norcia M, Retsema J, Strick CA, Su WG, Sutcliffe J, Wondrack L (1996) Glycylcyclines bind to the high-affinity tetracycline ribosomal binding site and evade Tet(M)- and Tet(O)-mediated ribosomal protection. Antimicrob Agents Chemother. 40(9):2226-8.

Blair $\mathrm{J}$ and Piddock L (2016) How to measure export via bacterial multidrug resistance efflux pumps. mBio 7(4) e00840-16. doi: 10.1128/mBio.00840-16.

Bolla JR, Howes AC, Fiorentino F, Robinson CV (2020) Assembly and regulation of the chlorhexidinespecific efflux pump Acel. Proc Natl Acad Sci USA.117(29):17011-17018. doi: 10.1073/pnas.2003271117.

Bradford PA, Petersen PJ, Young M, Jones CH, Tischler M, O'Connell J (2005) Tigecycline MIC testing by broth dilution requires use of fresh medium or addition of the biocatalytic oxygenreducing reagent oxyrase to standardize the test method. Antimicrob Agents Chemother. 49(9):3903-3909. doi: 10.1128/AAC.49.9.3903-3909.2005. 
Bradner WT (2001) Mitomycin C: a clinical update. Cancer Treat Rev 27:35-50. doi: 10.1053/ctrv.2000.0202.

Brown MH, Paulsen IT, Skurray RA (1999) The multidrug efflux protein NorM is a prototype of a new family of transporters. Mol Microbiol. 31(1):394-5. doi: 10.1046/j.1365-2958.1999.01162.x.

Brzoska AJ, Hassan KA, de Leon EJ, Paulsen IT, Lewis PJ (2013) Single-step selection of drug resistant Acinetobacter baylyi ADP1 mutants reveals a functional redundancy in the recruitment of multidrug efflux systems. PLoS One 8(2):e56090. doi: 10.1371/journal.pone.0056090.

Calvori C, Frontali L, Leoni L, Tecce G (1965) Effect of rifamycin on protein synthesis. Nature. 207(995):417-8. doi: 10.1038/207417a0.

Catel-Ferreira M, Coadou G, Molle V, Mugnier P, Nordmann P, Siroy A, Jouenne T, Dé E (2011) Structure-function relationships of CarO, the carbapenem resistance-associated outer membrane protein of Acinetobacter baumannii. J. Antimicrob. Chemother. 66:2053-2056. doi: 10.1093/jac/dkr267.

Catel-Ferreira M, Nehme R, Molle V, Aranda J, Bouffartigues E, Chevalier S, Bou G, Jouenne T, Dé E (2012) Deciphering the function of the outer membrane protein OprD homologue of Acinetobacter baumannii. Antimicrob. Agents Chemother. 56:3826-3832. doi:10.1128/AAC.06022-11.

CDC on Infectious Diseases in the United States: 1900-99. (1999) Population and Development Review, 25(3): 635-640. doi: 10.1111/j.1728-4457.1999.00635.x

Chang KC, Kuo HY, Tang CY, Chang CW, Lu CW, Liu CC, Lin HR, Chen KH, Liou ML (2014) Transcriptome profiling in imipenem-selected Acinetobacter baumannii. BMC Genomics. 26;15(1):815. doi: 10.1186/1471-2164-15-815.

Chau SL, Chu YW, Houang ET (2004) Novel resistance-nodulation-cell division efflux system AdeDE in Acinetobacter genomic DNA group 3. Antimicrob Agents Chemother 48:4054-5. doi: 10.1128/AAC.48.10.4054-4055.

Chellat MF, Raguž L, Riedl R (2016) Targeting antibiotic resistance. Angew Chem Int Ed Engl. 55(23):6600-26. doi: 10.1002/anie.201506818.

Chen Q, Li X, Zhou H, Jiang Y, Chen Y, Hua X, Yu Y (2014) Decreased susceptibility to tigecycline in Acinetobacter baumannii mediated by a mutation in trm encoding SAM-dependent methyltransferase. J Antimicrobial chemother 69:72-76. doi: 10.1093/jac/dkt319.

Chen YJ, Pornillos O, Lieu S, Ma C, Chen AP, Chang G (2007) X-ray structure of EmrE supports dual topology model. Proc Natl Acad Sci U S A. 104(48):18999-9004. doi: 10.1073/pnas.0709387104.

Chitsaz $\mathrm{M}$ and Brown MH (2017) The role played by drug efflux pumps in bacterial multidrug resistance. Essays Biochem 61(1): 127-139. doi: 10.1042/EBC20160064.

Chiu SK, Huang LY, Chen H, Tsai YK, Liou CH, Lin JC, Siu LK, Chang FY, Yeh KM (2017). Roles of ramR and tet(A) mutations in conferring tigecycline resistance in carbapenem-resistant Klebsiella pneumoniae clinical isolates. Antimicrob Agents Chemother. 61(8):e00391-17. doi: 10.1128/AAC.00391-17. 
Choi CH, Hyun SH, Lee JY, Lee JS, Lee YS, Kim SA, Chae JP, Yoo SM, Lee JC (2007) Acinetobacter baumannii outer membrane protein $A$ targets the nucleus and induces cytotoxicity. Cell Microbiol. 10:309-19. doi: 10.1111/j.1462-5822.2007.01041.x.

Choi CH, Lee JS, Lee YC, Park TI, Lee JC (2008) Acinetobacter baumannii invades epithelial cells and outer membrane protein A mediates interactions with epithelial cells. BMC Microbiol. 10(8):216. doi: 10.1186/1471-2180-8-216.

Chopra I and Roberts M (2001) Tetracycline antibiotics: mode of action, applications, molecular biology, and epidemiology of bacterial resistance. Microbiol Mol Biol Rev. 65: 232-260. doi:10.1128/MMBR.65.2.232-260.2001.

Chu YW, Chau SL, Houang ET (2006) Presence of active efflux systems AdeABC, AdeDE and AdeXYZ in different Acinetobacter genomic DNA groups. J Med Microbiol 55(4):477-8. doi: 10.1099/jmm.0.46433-0.

Clark RB (1996) Imipenem resistance among Acinetobacter baumannii: association with reduced expression of a 33-36 kDa outer membrane protein. J. Antimicrob Chemother. 38:245-251. doi: 10.1093/jac/38.2.245.

Connell SR, Tracz DM, Nierhaus KH, Taylor DE (2003) Ribosomal protection proteins and their mechanism of tetracycline resistance. Antimicrob Agents Chemother. 47(12):3675-81. doi: 10.1128/aac.47.12.3675-3681.2003.

Cortez-Cordova J, Kumar A (2011) Activity of the efflux pump inhibitor phenylalanine-arginine betanaphthylamide against the AdeFGH pump of Acinetobacter baumannii. Int. J. Antimicrob. Agents 37:420-424. doi: 10.1016/j.jjantimicag.2011.01.006.

Costa SF, Woodcock J, Gill M, Wise R, Barone AA, Caiaffa H, Levin AS (2000) Outer-membrane proteins pattern and detection of beta-lactamases in clinical isolates of imipenem-resistance Acinetobacter baumannii from Brazil. Int. J. Antimicrob. Agents 13:175-18. doi: 10.1016/s09248579(99)00123-5.

Coyne S, Guigon G, Courvalin P, Périchon B (2010a) Screening and quantification of the expression of antibiotic resistance genes in Acinetobacter baumannii with a microarray. Antimicrob. Agents Chemother. 54:333-340. doi: 10.1128/AAC.01037-09.

Coyne S, Rosenfeld N, Lambert T, Courvalin P, Perichon B (2010b) Overexpression of resistancenodulation-cell division pump AdeFGH confers multidrug resistance in Acinetobacter baumannii. Antimicrob. Agents Chemother. 54:4389-4393. doi: 10.1128/AAC.00155-10.

Coyne S, Courvalin P, Périchon B (2011) Efflux-mediated antibiotic resistance in Acinetobacter spp. Antimicrob Agents Chemother. 55(3):947-953. doi: 10.1128/AAC.01388-10.

Cruz-Muñiz MY, López-Jacome LE, Hernández-Durán M, Franco-Cendejas R, Licona-Limón P, Ramos-Balderas JL, Martinéz-Vázquez M, Belmont-Díaz JA, Wood TK, García-Contreras R (2017) Repurposing the anticancer drug mitomycin $C$ for the treatment of persistent Acinetobacter baumannii infections. Int J Antimicrob Agents 49: 88-92. doi: 10.1016/j.ijantimicag.2016.08.022. 
Damier-Piolle L, Magnet S, Bremont S, Lambert T, Courvalin P (2008) AdelJK, a resistancenodulation-cell division pump effluxing multiple antibiotics in Acinetobacter baumannii. Antimicrob. Agents Chemother. 52:557-562. doi: 10.1128/AAC.00732-07.

de Cristóbal RE, Vincent PA, Salomón RA (2006) Multidrug resistance pump AcrAB-TolC is required for high-level, Tet(A)-mediated tetracycline resistance in Escherichia coli. J Antimicrob Chemother. 58: 31-6. doi: 10.1093/jac/dkl172.

del Mar Tomás M, Beceiro A, Pérez A, Velasco D, Moure R, Villanueva R, Martínez-Beltrán J, Bou G (2005) Cloning and functional analysis of the gene encoding the 33- to 36 kilodalton outer membrane protein associated with carbapenem resistance in Acinetobacter baumannii. Antimicrob Agents Chemother. 49(12):5172-5. doi: 10.1128/AAC.49.12.5172-5175.2005.

Dijkshoorn L, Nemec A, Seifert H (2007) An increasing threat in hospitals: multidrug-resistant Acinetobacter baumannii. Nat Rev Microbiol. 5(12):939-51. doi: 10.1038/nrmicro1789.

Dinos GP, Athanassopoulos CM, Missiri DA, Giannopoulou PC, Vlachogiannis IA, Papadopoulos GE, Papaioannou D, Kalpaxis DL (2016) Chloramphenicol derivatives as antibacterial and anticancer agents: historic problems and current solutions. Antibiotics (Basel) 5(2):20. doi: 10.3390/antibiotics5020020.

Dönhöfer A, Franckenberg S, Wickles S, Berninghausen O, Beckmann R, Wilson DN (2012) Structural basis for TetM-mediated tetracycline resistance. Proc Natl Acad Sci U S A. 16;109(42):16900-5. doi: 10.1073/pnas.1208037109.

Drlica K, Zhao X (1997) DNA gyrase, topoisomerase IV, and the 4-quinolones. Microbiol Mol Biol Rev. 61(3):377-92.

Drlica K, Zhao X, Malik M, Salz T, Kerns R (2012) Fluoroquinolone Resistance: Mechanisms, Restrictive Dosing, and Anti-Mutant Screening Strategies for New Compounds. In: Dougherty T., Pucci M. (eds) Antibiotic Discovery and Development. Springer, Boston, MA. doi: 10.1007/978-14614-1400-1_14

Du D, Wang-Kan X, Neuberger A, van Veen HW, Pos KM, Piddock LJV, Luisi BF (2018) Multidrug efflux pumps: structure, function and regulation. Nat Rev Microbiol. 16(9):523-539. doi: 10.1038/s41579-018-0048-6.

Du X, He F, Shi Q, Zhao F, Xu J, Fu Y, Yu Y (2018) The rapid emergence of tigecycline resistance in blakPC-2 harboring Klebsiella pneumoniae, as mediated in Vivo by mutation in tetA during tigecycline treatment. Front Microbiol. 9:648. doi: 10.3389/fmicb.2018.00648.

Dupont M, Pages JM, Lafitte D, Siroy A, Bollet C (2005) Identification of an OprD homologue in Acinetobacter baumannii. J. Proteome Res. 4:2386-2390. doi:10.1021/pr050143q.

Durand GA, Raoult D, Dubourg G (2019) Antibiotic discovery: history, methods and perspectives. International Journal of Antimicrobial Agents. 53(4):371-382. doi: 10.1016/j.jjantimicag.2018.11.010.

Ehrlich J, Bartz QR, Smith RM, Joslyn DA, Burkholder PR (1947) Chloromycetin, a New Antibiotic From a Soil Actinomycete. Science. 106(2757):417. doi: 10.1126/science.106.2757.417. 
El-Shazly S, Dashti A, Vali L, Bolaris M, Ibrahim AS (2015) Molecular epidemiology and characterization of multiple drug-resistant (MDR) clinical isolates of Acinetobacter baumannii. Int $\mathrm{J}$ Infect Dis. 41:42-9. doi: 10.1016/j.ijid.2015.10.016.

Emmerson AM and Jones AM (2003) The quinolones: decades of development and use. J Antimicrob Chemother. 51 Suppl 1:13-20. doi: 10.1093/jac/dkg208.

Falagas ME and Rafailidis PI (2007) Attributable mortality of Acinetobacter baumannii: no longer a controversial issue. Crit Care. 11(3):134. doi: 10.1186/cc5911.

Fawzi MB, Zhu T, Shah SM (2011) Tigecycline compositions and methods of preparation. United States patent US. 7,879,828.

Fernández-Cuenca F, Smani Y, Gomez-Sanchez MC, Docobo-Perez F, Caballero-Moyana FJ, Dominguez-Herrera J, Pascual A, Pachon J (2011) Attenuated virulence of a slow-growing pandrug-resistant Acinetobacter baumannii is associated with decreased expression of genes encoding the porins CarO and OprD-like. Int. J. Antimicrob. Agents 38:548-549. doi: 10.1016/j.ijantimicag.2011.08.002.

Fernández-Villa D, Aguilar MR, Rojo L (2019) Folic acid antagonists: Antimicrobial and immunomodulating mechanisms and applications. Int J Mol Sci. 20(20):4996. doi: 10.3390/ijms20204996.

Fitzpatrick AWP, Llabrés S, Neuberger A, Blaza JN, Bai XC, Okada U, Murakami S, van Veen HW, Zachariae U, Scheres SHW, Luisi BF, Du D (2017) Structure of the MacAB-TolC ABC-type tripartite multidrug efflux pump. Nat Microbiol. 2:17070. doi: 10.1038/nmicrobiol.2017.70.

Fleishman SJ, Harrington SE, Enosh A, Halperin D, Tate CG, Ben-Tal N (2006) Quasi-symmetry in the cryo-EM structure of EmrE provides the key to modeling its transmembrane domain. Journal of Molecular Biology 364(1):54-67. doi: 10.1016/j.jmb.2006.08.072.

Fluman N, Bibi E (2009) Bacterial multidrug transport through the lens of the major facilitator superfamily. Biochim Biophys Acta 1794(5):738-47. doi: 10.1016/j.bbapap.2008.11.020.

Fluman N, Ryan CM, Whitelegge JP, Bibi E (2012) Dissection of mechanistic principles of a secondary multidrug efflux protein. Mol Cell. 47(5):777-87. doi: 10.1016/j.molcel.2012.06.018.

Fluman N, Adler J, Rotenberg S, Brown MH, Bibi E (2014) Export of a single drug molecule in two transport cycles by a multidrug efflux pump. Nat Commun 5: 4615. doi: 10.1038/ncomms5615.

Fluman N, Tobiasson V, von Heijne G (2017) Stable membrane orientations of small dual-topology membrane proteins. Proc Natl Acad Sci USA. 114(30):7987-7992. doi: 10.1073/pnas.1706905114.

Fournier PE, Vallenet D, Barbe V, Audic S, Ogata H, Poirel L, Richet H, Robert C, Mangenot S, Abergel C, Nordmann P, Weissenbach J, Raoult D, Claverie JM (2006) Comparative genomics of multidrug resistance in Acinetobacter baumannii. PLoS Genet. 2(1):e7. doi: 10.1371/journal.pgen.0020007.

Frech G, Schwarz S (1999) Plasmid-encoded tetracycline resistance in Salmonella enterica subsp. enterica serovars choleraesuis and typhimurium: identification of complete and truncated Tn1721 elements. FEMS Microbiol Lett. 176(1):97-103. doi: 10.1111/j.1574-6968.1999.tb13648.x. 
Gaddy JA, Tomaras AP, Actis LA (2009) The Acinetobacter baumannii 19606 OmpA protein plays a role in biofilm formation on abiotic surfaces and in the interaction of this pathogen with eukaryotic cells. Infect. Immun. 77(8):3150-3160. doi:10.1128/IAI.00096-09.

Gallagher LA, Ramage E, Weiss EJ, Radey M, Hayden HS, Held KG, Hus HK, Zurawski DV, Brittnacher MJ, Manoil C (2015) Resources for genetic and genomic analysis of emerging pathogen Acinetobacter baumannii. J Bacteriol 197:2027-2035. doi:10.1128/JB.00131-15.

Gerson S, Nowak J, Zander E, Ertel J, Wen Y, Krut O, Seifert H, Higgins PG (2018) Diversity of mutations in regulatory genes of resistance-nodulation-cell division efflux pumps in association with tigecycline resistance in Acinetobacter baumannii. J Antimicrob Chemother. 73(6):1501-1508. doi: 10.1093/jac/dky083.

Giamarellou H and Poulakou G (2011) Pharmacokinetic and pharmacodynamic evaluation of tigecycline. Expert Opin Drug Metab Toxicol 7(11): 1459-1470. doi: 10.1517/17425255.2011.623126.

Gibson DG, Young L, Chuang RY, Venter JC, Hutchison CA 3rd, Smith HO (2009) Enzymatic assembly of DNA molecules up to several hundred kilobases. Nat Methods. 2009 May;6(5):343-5. doi: 10.1038/nmeth.1318.

Godeux AS, Svedholm E, Lupo A, Haenni M, Venner S, Laaberki MH, Charpentier X (2020) Scarless removal of large resistance island AbaR results in antibiotic susceptibility and increased natural transformability in Acinetobacter baumannii. Antimicrob Agents Chemother. 64(10):e00951-20. doi: 10.1128/AAC.00951-20.

Göttig S, Gruber TM, Higgins PG, Wachsmuth M, Seifert H, Kempf VA (2014) Detection of pan drugresistant Acinetobacter baumannii in Germany. J Antimicrob Chemother. 69(9):2578-9. doi: 10.1093/jac/dku170.

Granados-Chinchilla F, Rodríguez C (2017) Tetracyclines in food and feeding stuffs: from regulation to analytical methods, bacterial resistance, and environmental and health implications. J Anal Methods Chem.1315497. doi: 10.1155/2017/1315497.

Greene NP, Kaplan E, Crow A, Koronakis V (2018) Antibiotic resistance mediated by the MacB ABC transporter family: a structural and functional perspective. Front Microbiol. 9:950. doi: 10.3389/fmicb.2018.00950.

Groisman EA (2016) Feedback control of two-component regulatory systems. Annu. Rev. Microbiol. 70:103-124. doi:10.1146/annurev-micro-102215-095331.

Grossman TH, Starosta AL, Fyfe C, O'Brien W, Rothstein DM, Mikolajka A, Wilson DN, Sutcliffe JA (2012) Target- and resistance-based mechanistic studies with TP-434, a novel fluorocycline antibiotic. Antimicrob Agents Chemother. 56(5):2559-2564. doi:10.1128/AAC.06187-11. Erratum in: Antimicrob Agents Chemother (2015) 59(9):5870. doi:10.1128/AAC.01551-15.

Guay GG, Khan SA, Rothstein DM (1993) The tet(K) gene of plasmid pT181 of Staphylococcus aureus encodes an efflux protein that contains 14 transmembrane helices. Plasmid 30(2):163-6. doi: 10.1006/plas.1993.1045. 
Hamidian M, Holt KE, Pickard D, Hall RM (2016) A small Acinetobacter plasmid carrying the tet39 tetracycline resistance determinant. J Antimicrob Chemother. 71:269-271. doi: 10.1093/jac/dkv293.

Hassan KA, Jackson SM, Penesyan A, Patching SG, Tetu SG, Eijkelkamp BA, Brown MH, Henderson PJ, Paulsen IT (2013) Transcriptomic and biochemical analyses identify a family of chlorhexidine efflux proteins. Proc Natl Acad Sci USA 110(50):20254-9. doi: 10.1073/pnas.1317052110.

Hassan KA, Liu Q, Elbourne LDH, Ahmad I, Sharples D, Naidu V, Chan CL,Li L, Harborne SPD, Pokhrel A, Postis VLG, Goldman A, Henderson PJ, Paulsen IT (2018) Pacing across the membrane: the novel PACE family of efflux pumps is widespread in Gram-negative pathogens. Res Microbiol. 169:450-454. doi: 10.1016/j.resmic.2018.01.001.

Hassan KA, Liu Q, Henderson PJ, Paulsen IT (2015) Homologs of the Acinetobacter baumannii Acel transporter represent a new family of bacterial multidrug efflux systems. mBio. 10; 6(1):e0198214. doi: $10.1128 / \mathrm{mBio} .01982-14$.

Hassan KA, Naidu V, Edgerton JR, Mettrick KA, Liu Q, Fahmy L, Li L, Jackson SM, Ahmad I, Sharples D, Henderson PJF, Paulsen IT (2019) Short-chain diamines are the physiological substrates of PACE family efflux pumps. Proc Natl Acad Sci USA 116(36):18015-18020. doi: 10.1073/pnas.1901591116.

He T, Wang R, Liu D, Walsh TR, Zhang R, Lv Y, Ke Y, Ji Q, Wei R, Liu Z, Shen Y, Wang G, Sun L, Lei L, Lv Z, Li Y, Pang M, Wang L, Sun Q, Fu Y, Song H, Hao Y, Shen Z, Wang S, Chen G, Wu C, Shen J, Wang $Y$ (2019) Emergence of plasmid-mediated high-level tigecycline resistance genes in animals and humans. Nat Microbiol. 4(9):1450-1456. doi: 10.1038/s41564-019-0445-2.

He T, Wei R, Li R, Zhang L, Sun L, Bao H, Gu J, Wang Y, Wang R (2020) Co-existence of tet(X4) and mcr-1 in two porcine Escherichia coli isolates. J Antimicrob Chemother. 75(3):764-766. doi: 10.1093/jac/dkz510.

He X, Lu F, Yuan F, Jiang D, Zhao P, Zhu J, Cheng H, Cao J, Lu G (2015) Biofilm formation caused by clinical Acinetobacter baumannii isolates is associated with overexpression of the AdeFGH efflux pump. Antimicrob Agents Chemother. 59(8):4817-25. doi: 10.1128/AAC.00877-15.

He X, Szewczyk P, Karyakin A, Evin M, Hong WX, Zhang Q, Chang G (2010) Structure of a cationbound multidrug and toxic compound extrusion transporter. Nature 467(7318):991-4. doi: 10.1038/ nature09408.

Heng J, Zhao Y, Liu M, Liu Y, Fan J, Wang X, Zhao Y, Zhang XC (2015) Substrate-bound structure of the E. coli multidrug resistance transporter MdfA. Cell Res. 25(9):1060-73. doi: 10.1038/cr.2015.94.

Hentschke M, Christner M, Sobottka I, Aepfelbacher M, Rohde H (2010) Combined ramR mutation and presence of a $\operatorname{Tn} 1721$-associated $\operatorname{tet}(A)$ variant in a clinical isolate of Salmonella enterica serovar Hadar resistant to tigecycline. Antimicrob Agents Chemother. 54(3):1319-1322. doi:10.1128/AAC.00993-09.

Hillen W and Berens C (1994) Mechanisms underlying expression of Tn10 encoded tetracycline resistance. Annu Rev Microbiol. 48:345-69. 
Hirata T, Saito A, Nishino K, Tamura N, Yamaguchi A (2004) Effects of efflux transporter genes on susceptibility of Escherichia coli to tigecycline (GAR-936). Antimicrob Agents Chemother. 48(6):2179-2184. doi:10.1128/AAC.48.6.2179-2184.2004.

Holdsworth SR, Law CJ (2012) Functional and biochemical characterisation of the Escherichia coli major facilitator superfamily multidrug transporter MdtM. Biochimie 94:1334-1346.

Hope R, Warner M, Mushtaq S, Ward ME, Parsons T, Livermore DM (2005) Effect of medium type, age and aeration on the MICs of tigecycline and classical tetracyclines. J Antimicrobial Chemother. 56(6):1042-1046. doi.org/10.1093/jac/dki386.

Horiyama T, Nikaido E, Yamaguchi A, Nishino K (2011) Roles of Salmonella multidrug efflux pumps in tigecycline resistance. J Antimicrobial Chemother. 66(1):105-110. doi.org/10.1093/jac/dkq421.

Hornsey M, Ellington MJ, Doumith M, Thomas CP, Gordon NC, Wareham DW, Quinn J, Lolans K, Livermore DM, Woodford N (2010) AdeABC-mediated efflux and tigecycline MICs for epidemic clones of Acinetobacter baumannii. J Antimicrob Chemother 65(8):1589-93. doi:10.1093/jac/dkq218.

Hornsey M, Loman N, Wareham DW, Ellington MJ, Pallen MJ, Turton JF, Underwood A, Gaulton T, Thomas CP, Doumith M, Livermore DM, Woodford N (2011) Whole-genome comparison of two Acinetobacter baumannii isolates from a single patient, where resistance developed during tigecycline therapy. J Antimicrobial Chemother. 66(7): 1499-1503. doi: 10.1093/jac/dkr168.

Hornsey M and Wareham DW (2018) Effects of in vivo emergent tigecycline resistance on the pathogenic potential of Acinetobacter baumannii. Sci Rep 8:4234. doi: 10.1038/s41598-01822549-6.

Hou PF, Chen XY, Yan GF, Wang YP, Ying CM (2012) Study of the correlation of imipenem resistance with efflux pumps AdeABC, AdelJK, AdeDE and AbeM in clinical isolates of Acinetobacter baumannii. Chemotherapy. 58:152-158. doi: 10.1159/000335599.

Hua X, Chen Q, Li X, Yu Y (2014) Global transcriptional response of Acinetobacter baumannii to a subinhibitory concentration of tigecycline. Int $\mathrm{J}$ Antimicrob Agents 44:337-344. doi: 10.1016/j.ijantimicag.2014.06.015.

Hugh R and Reese R (1967) Designation of the type strain for Bacterium anitratum Schaub and Hauber 1948. Int. J. Syst. Evol. Microbiol. 17, 245-254.

Huys G, Cnockaert M, Vaneechoutte M, Woodford N, Nemec A, Dijkshoorn L, Swings J (2005) Distribution of tetracycline resistance genes in genotypically related and unrelated multiresistant Acinetobacter baumannii strains from different European hospitals. Res. Microbiol. 156:348-355. doi: 10.1016/j.resmic.2004.10.008.

lacono M, Villa L, Fortini D, Bordoni R, Imperi F, Bonnal RJ, Sicheritz-Ponten T, De Bellis G, Visca P, Cassone A, Carattoli A (2008) Whole-genome pyrosequencing of an epidemic multidrug-resistant Acinetobacter baumannii strain belonging to the European clone II group. Antimicrob Agents Chemother. 52(7):2616-25. doi: 10.1128/AAC.01643-07. 
lyer R, Moussa SH, Durand-Réville TF, Tommasi R, Miller A (2018) Acinetobacter baumannii OmpA is a selective antibiotic permeant porin. ACS Infectious Diseases 4(3):373-381. doi: 10.1021/acsinfecdis.7b00168.

lyer VN and Szybalski (1964) Mitomycins and porfiromycin: chemical mechanism of activation and cross-linking of DNA. Science 145(627): 55-58. doi: 10.1126/science.145.3627.55.

Jardetzky O (1966) Simple allosteric model for membrane pumps. Nature. 211(5052):969-70. doi: 10.1038/211969a0.

Jenner L, Starosta AL, Terry DS, Mikolajka A, Filonava L, Yusupov M, Blanchard SC, Wilson DN, Yusupova G (2013) Structural basis for potent inhibitory activity of the antibiotic tigecycline during protein synthesis. Proc Natl Acad Sci USA. 110(10):3812-6. doi: 10.1073/pnas.1216691110.

Jin JS, Kwon SO, Moon DC, Gurung M, Lee JH, Kim SI, Lee JC (2011) Acinetobacter baumannii secretes cytotoxic outer membrane protein A via outer membrane vesicles. PLoS One. 28;6(2):e17027. doi: 10.1371/journal.pone.0017027.

Jyothisri K, Deepak V, Rajeswari MR (1999) Purification and characterization of a major $40 \mathrm{kDa}$ outer membrane protein of Acinetobacter baumannii. FEBS Lett. 443:57-60. doi: 10.1016/s00145793(98)01679-2.

Karalewitz AP, Miller SI (2018) Multidrug-resistant Acinetobacter baumannii chloramphenicol resistance requires an inner membrane permease. Antimicrob Agents Chemother. 62(8):e0051318. doi: 10.1128/AAC.00513-18.

Kobayashi N, Nishino K, Yamaguchi A (2001) Novel macrolide-specific ABC-type efflux transporter in Escherichia coli. J Bacteriol 183: 5639-5644. doi:10.1128/JB.183.19.5639-5644.2001.

Korkhov VM and Tate CG (2009) An emerging consensus for the structure of EmrE. Acta Crystallogr D Biol Crystallogr. 65(Pt 2):186-92. doi: 10.1107/S0907444908036640.

Koronakis V, Sharff A, Koronakis E, Luisi B, Hughes C (2000) Crystal structure of the bacterial membrane protein TolC central to multidrug efflux and protein export. Nature 405: 914-919. doi: $10.1038 / 35016007$.

Kuroda T and Tsuchiya T (2009) Multidrug efflux transporters in the MATE family. Biochimica et Biophysica Acta. 1794(5):763-768. doi: 10.1016/j.bbapap.2008.11.012.

Kusakizako T, Miyauchi H, Ishitani R, Nureki O (2020) Structural biology of the multidrug and toxic compound extrusion superfamily transporters. Biochimica et Biophysica Acta (BBA) Biomembranes 1862: 183154. doi: 10.1016/j.bbamem.2019.183154.

Kwan BW, Chowdhury N, Wood TK (2015) Combatting bacterial infections by killing persister cells with mitomycin C. Environ Microbiol 17:4406-14. doi: 10.1111/1462-2920.12873.

Kwon HI, Kim S, Oh MH, Na SH, Kim YJ, Jeon YH, Lee JC (2017) Outer membrane protein A contributes to antimicrobial resistance of Acinetobacter baumannii through the OmpA-like domain. J. Antimicrob. Chemother. 72(11):3012-3015. doi: 10.1093/jac/dkx257.

Lee A, Mao W, Warren MS, Mistry A, Hoshino K, Okumura R, Ishida H, Lomovskaya O (2000) Interplay between efflux pumps may provide either additive or multiplicative effects on drug resistance. J Bacteriol 182: 3142-50. doi: 10.1128/JB.182.11.3142-3150.2000. 
Lee HY, Chang RC, Su LH, Liu SY, Wu DR, Chuang CH, Chen CL, Chiu CH (2012) Wide spread of Tn2006 in an AbaR4-type resistance island among carbapenem-resistant Acinetobacter baumannii clinical isolates in Taiwan. Int $J$ Antimicrob Agents. 40:163-167. doi: 10.1016/j.ijantimicag.2012.04.018.

Lee JS, Choi CH, Kim JW, Lee JC (2010) Acinetobacter baumannii outer membrane protein A induces dendritic cell death through mitochondrial targeting. J. Microbiol. 48:387-392. doi: 10.1007/s12275-010-0155-1.

Lee Y, Kim CK, Lee H, Jeong SH, Yong D, Lee K (2011) A novel insertion sequence, ISAba10, inserted into ISAba1 adjacent to the bla(OXA-23) gene and disrupting the outer membrane protein gene carO in Acinetobacter baumannii. Antimicrob Agents Chemother. 55(1):361-3. doi: 10.1128/AAC.01672-09.

Leninger M, Sae Her A, Traaseth NJ (2019) Inducing conformational preference of the membrane protein transporter EmrE through conservative mutations. Elife. 8:e48909. doi: 10.7554/eLife.48909.

Leus IV, Weeks JW, Bonifay V, Smith L, Richardson S, Zgurskaya HI (2018) Substrate specificities and efflux efficiencies of RND efflux pumps of Acinetobacter baumannii. J Bacteriol. 200(13):e00049-18. doi: 10.1128/JB.00049-18.

Leus IV, Adamiak J, Trinh AN, Smith L, Richardson S, Ernst R, Zgurskaya HI (2020) Inactivation of AdeABC and AdelJK efflux pumps elicits specific nonoverlapping transcriptional and phenotypic responses in Acinetobacter baumannii. Mol Microbiol. 00: 1-17. doi: 10.1111/mmi.14594.

Levy SB, McMurry L (1978) Plasmid-determined tetracycline resistance involves new transport systems for tetracycline. Nature 276:90-92.

Lewinson O, Adler J, Poelarends GJ, Mazurkiewicz P, Driessen AJ, Bibi E (2003) The Escherichia coli multidrug transporter MdfA catalyzes both electrogenic and electroneutral transport reactions. Proc Natl Acad Sci USA. 100(4):1667-72. doi: 10.1073/pnas.0435544100.

Lewinson O, Padan E, Bibi E (2004) Alkalitolerance: a biological function for a multidrug transporter in pH homeostasis. Proc Natl Acad Sci USA. 101(39):14073-8. doi: 10.1073/pnas.0405375101.

Li H, Luo YF, Williams BJ, Blackwell TS, Xie CM (2012) Structure and function of OprD protein in Pseudomonas aeruginosa: from antibiotic resistance to novel therapies. Int $\mathrm{J}$ Med Microbiol. 302(2):63-8. doi: 10.1016/j.jjmm.2011.10.001.

Li W, Atkinson GC, Thakor NS, Allas U, Lu CC, Chan KY, Tenson T, Schulten K, Wilson KS, Hauryliuk V, Frank J (2013) Mechanism of tetracycline resistance by ribosomal protection protein Tet(O). Nat Commun. 4:1477. doi: 10.1038/ncomms2470.

Lin HT, Bavro VN, Barrera NP, Frankish HM, Velamakanni S, van Veen HW, Robinson CV, BorgesWalmsley MI, Walmsley AR (2009a) MacB ABC transporter is a dimer whose ATPase activity and macrolide-binding capacity are regulated by the membrane fusion protein MacA. J Biol Chem. 284(2):1145-54. doi: 10.1074/jbc.M806964200. 
Lin L, Ling BD, Li XZ (2009b) Distribution of the multidrug efflux pump genes, ade $A B C$, adeDE and adelJK, and class 1 integron genes in multiple-antimicrobial-resistant clinical isolates of Acinetobacter baumannii-Acinetobacter calcoaceticus complex. Int J Antimicrob Agents 33(1):2732. doi: 10.1016/j.jijantimicag.2008.06.027.

Lin MF, Lin YY, Tu CC, Lan CY (2015) Distribution of different efflux pump genes in clinical isolates of multidrug-resistant Acinetobacter baumannii and their correlation with antimicrobial resistance. $\mathrm{J}$ Microb Immunol Infect 50:224-231. doi: 10.1016/j.jmii.2015.04.004.

Linkevicius M, Sandegren L, Andersson DI (2016) Potential of tetracycline resistance proteins to evolve tigecycline resistance. Antimicrob Agents Chemother 60: 789-96. doi: 10.1128/AAC.0246515.

Liu L, Cui Y, Zheng B, Jiang S, Yu W, Shen P, Ji J, Li L, Qin N, Xiao Y (2016) Analysis of tigecycline resistance development in clinical Acinetobacter baumannii isolates through a combined genomic and transcriptomic approach. Sci Rep. 6:26930. doi: 10.1038/srep26930.

Liu Q, Hassan KA, Ashwood HE, Gamage HKAH, Li L, Mabbutt BC, Paulsen IT (2018) Regulation of the acel multidrug efflux pump gene in Acinetobacter baumannii. J Antimicrob Chemother. 73(6):1492-1500. doi: 10.1093/jac/dky034.

Locher KP (2009) Structure and mechanism of ATP-binding cassette transporters. Philos Trans R Soc Lond B Biol Sci. 364(1514):239-45. doi: 10.1098/rstb.2008.0125.

Lu M, Radchenko M, Symersky J, Nie R, Guo Y (2013b) Structural insights into $\mathrm{H}^{+}$-coupled multidrug extrusion by a MATE transporter. Nat Struct Mol Biol. 20(11):1310-7. doi: 10.1038/nsmb.2687.

Lu M, Symersky J, Radchenko M, Koide A, Guo Y, Nie R, Koide S (2013a) Structures of a $\mathrm{Na}^{+}-$ coupled, substrate-bound MATE multidrug transporter. Proc Natl Acad Sci USA 110(6):2099-104. doi: 10.1073/pnas.1219901110.

Luo L, Jiang X, Wu Q, Wei L, Li J, Ying C (2011) Efflux pump overexpression in conjunction with alternation of outer membrane protein may induce Acinetobacter baumannii resistant to imipenem. Chemotherapy 57:77-84. doi: 10.1159/000323620.

Luo G, Lin L, Ibrahim AS, Baquir B, Pantapalangkoor P, Bonomo RA, Doi Y, Adams MD, Russo TA, Spellberg B (2012) Active and passive immunization protects against lethal, extreme drug resistant-Acinetobacter baumannii infection. PLoS One 7(1):e29446. doi: 10.1371/journal.pone.

Lytvynenko I, Brill S, Oswald C, Pos KM (2016) Molecular basis of polyspecificity of the Small Multidrug Resistance efflux pump AbeS from Acinetobacter baumannii. Journal of Molecular Biology 428(3):644-657. doi: 10.1016/j.jmb.2015.12.006.

Ma P, Varela F, Magoch M, Silva AR, Rosário AL, Brito J, Oliveira TF, Nogly P, Pessanha M, Stelter M, Kletzin A, Henderson PJ, Archer M (2013) An efficient strategy for small-scale screening and production of archaeal membrane transport proteins in Escherichia coli. PLoS One. 8(10):e76913. doi: 10.1371/journal.pone.0076913.

Magnet S, Courvalin P, Lambert T (2001) Resistance-nodulation-cell division-type efflux pump involved in aminoglycoside resistance in Acinetobacter baumannii strain BM4454. Antimicrob Agents Chemother. 45(12):3375-80. doi: 10.1128/AAC.45.12.3375-3380.2001. 
Mahillon J and Chandler M (1998) Insertion sequences. Microbiol Mol Biol Rev. 62(3):725-74.

Marchand I, Damier-Piolle L, Courvalin P, and Lambert T (2004) Expression of the RND-type efflux pump AdeABC in Acinetobacter baumannii is regulated by the AdeRS two-component system. Antimicrob. Agents Chemother. 48:3298-3304. doi: 10.1128/AAC.48.9.3298-3304.2004.

Masi M, Réfregiers M, Pos KM, Pagès JM (2017) Mechanisms of envelope permeability and antibiotic influx and efflux in Gram-negative bacteria. Nat Microbiol. 2:17001. doi: 10.1038/nmicrobiol.2017.1.

McMurry L, Petrucci RE Jr, Levy SB (1980) Active efflux of tetracycline encoded by four genetically different tetracycline resistance determinants in Escherichia coli. Proc Natl Acad Sci USA. 77(7):3974-7. doi: 10.1073/pnas.77.7.3974.

Mendling W, Weissenbacher ER, Gerber S, Prasauskas V, Grob P (2016) Use of locally delivered dequalinium chloride in the treatment of vaginal infections: a review. Arch Gynecol Obstet. 293(3):469-84. doi: 10.1007/s00404-015-3914-8.

Montaña S, Vilacoba E, Traglia GM, Almuzara M, Pennini M, Fernández A, Sucari A, Centrón D, Ramírez MS (2015) Genetic variability of AdeRS two-component system associated with tigecycline resistance in XDR-Acinetobacter baumannii isolates. Curr. Microbiol. 71:76-82. doi: 10.1007/s00284-015-0829-3.

Morris GM, Huey R, Lindstrom W, Sanner MF, Belew RK, Goodsell DS, Olson AJ (2009) AutoDock4 and AutoDockTools4: Automated docking with selective receptor flexibility. J Comput Chem. 30(16):2785-91. doi: 10.1002/jcc.21256.

Morrison EA, DeKoster GT, Dutta S, Vafabakhsh R, Clarkson MW, Bahl A, Kern D, Ha T, HenzlerWildman KA (2011) Antiparallel EmrE exports drugs by exchanging between asymmetric structures. Nature 481(7379):45-50. doi: 10.1038/nature10703.

Mortimer PG and Piddock LJ (1991) A comparison of methods used for measuring the accumulation of quinolones by Enterobacteriaceae, Pseudomonas aeruginosa and Staphylococcus aureus. J Antimicrob Chemother. 28(5):639-53. doi: 10.1093/jac/28.5.639

Mousa JJ and Bruner SD (2016) Structural and mechanistic diversity of multidrug transporters. Nat Prod Rep. 33(11):1255-1267. doi: 10.1039/c6np00006a.

Mugnier PD, Poirel L, Nordmann P (2009) Functional analysis of insertion sequence ISAba1, responsible for genomic plasticity of Acinetobacter baumannii. J Bacteriol. 191(7):2414-18. doi: 10.1128/JB.01258-08.

Murakami S, Nakashima R, Yamashita E, Yamaguchi A (2002) Crystal structure of bacterial multidrug efflux transporter AcrB. Nature 419(6907):587-93. doi: 10.1038/nature01050.

Murray IA, Shaw WV (1997) O-Acetyltransferases for chloramphenicol and other natural products. Antimicrob Agents Chemother. 41(1):1-6. doi: 10.1128/AAC.41.1.1.

Mussi MA, Limansky AS, Viale AM (2005) Acquisition of resistance to carbapenems in multidrugresistant clinical strains of Acinetobacter baumannii: natural insertional inactivation of a gene encoding a member of a novel family of beta-barrel outer membrane proteins. Antimicrob Agents Chemother. 49(4):1432-40. doi: 10.1128/AAC.49.4.1432-1440.2005. 
Mussi MA, Relling VM, Limansky AS, Viale AM (2007) CarO, an Acinetobacter baumannii outer membrane protein involved in carbapenem resistance, is essential for $L$-ornithine uptake. FEBS Lett. 581:5573-5578. doi: 10.1016/j.febslet.2007.10.063.

Muth T and Schuldiner S (2000) A membrane embedded glutamate is required for ligand binding to the multidrug transporter EmrE. The EMBO Journal 19:234-240. doi: 10.1093/emboj/19.2.234.

Nagarathinam K, Nakada-Nakura Y, Parthier C, Terada T, Juge N, Jaenecke F, Liu K, Hotta Y, Miyaji T, Omote H, Iwata S, Nomura N, Stubbs MT, Tanabe M (2018) Outward open conformation of a Major Facilitator Superfamily multidrug/ $\mathrm{H}^{+}$antiporter provides insights into switching mechanism. Nat Commun. 9(1):4005. doi: 10.1038/s41467-018-06306-x.

Nair AV, Lee KW, van Veen HW (2016) Structural and functional landscape of MFS and MATE efflux pumps. In: Li XZ, Elkins C, Zgurskaya H. (eds) Efflux-Mediated Antimicrobial Resistance in Bacteria. Springer International Publishing, 29-44. doi: 10.1007/978-3-319-39658-3_2.

Nelson ML and Levy SB (2011) The history of the tetracyclines. Annals of the New York Academy of Sciences 1241(1): 17-32. doi: 10.1111/j.1749-6632.2011.06354.x.

Nemec A, Maixnerova M, van der Reijden TJ, van den Broek PJ, Dijkshoorn L (2007) Relationship between the AdeABC efflux system gene content, netilmicin susceptibility and multidrug resistance in a genotypically diverse collection of Acinetobacter baumannii strains. J. Antimicrob. Chemother. 60:483-489. doi: 10.1093/jac/dkm231.

Nguyen F, Starosta AL, Arenz S, Sohmen D, Dönhöfer A, Wilson DN (2014) Tetracycline antibiotics and resistance mechanisms. Biological Chemistry. 395(5):559-575. doi: 10.1515/hsz-2013-0292.

Nie D, Hu Y, Chen Z, Li M, Hou Z, Lio X, Mao X, Xue X (2020) Outer membrane protein A (OmpA) as a potential therapeutic target for Acinetobacter baumannii infection. J Biomed Sci 27:26. doi: 10.1186/s12929-020-0617-7.

Nikaido $H$ (1989) Outer membrane barrier as a mechanism of antimicrobial resistance. Antimicrob Agents Chemother. 33(11):1831-6. doi: 10.1128/aac.33.11.1831.

Nikaido H (1996) Multidrug efflux pumps of gram-negative bacteria. J Bacteriol. 178(20):5853-9. doi: 10.1128/jb.178.20.5853-5859.1996.

Nikaido H (2003) Molecular basis of bacterial outer membrane permeability revisited. Microbiol Mol. Biol. Rev. 67 (4):593-656. doi: 10.1128/mmbr.67.4.593-656.2003.

Nikaido $\mathrm{H}$ and Pagès JM (2012) Broad-specificity efflux pumps and their role in multidrug resistance of Gram-negative bacteria. FEMS Microbiol Rev. 36(2):340-63. doi: 10.1111/j.15746976.2011.00290.x.

Nolivos S, Cayron J, Dedieu A, Page A, Delolme F, Lesterlin C (2019) Role of AcrAB-TolC multidrug efflux pump in drug-resistance acquisition by plasmid transfer. Science 364:778-82. doi: 10.1126/science.aav6390.

Novović K, Mihajlović S, Dinić M, Malešević M, Miljković M, Kojić M, Jovčić B (2018) Acinetobacter spp. porin Omp33-36: Classification and transcriptional response to carbapenems and host cells. PLoS One 13(8):e0201608. doi: 10.1371/journal.pone.0201608. 
Okada U, Yamashita E, Neuberger A, Morimoto M, van Veen HW, Murakami S (2017) Crystal structure of tripartite-type $A B C$ transporter MacB from Acinetobacter baumannii. Nat Commun. 8(1):1336. doi: 10.1038/s41467-017-01399-2.

Olson MW, Ruzin A, Feyfant E, Rush TS 3rd, O'Connell J, Bradford PA (2006) Functional, biophysical, and structural bases for antibacterial activity of tigecycline. Antimicrob Agents Chemother. 50(6):2156-66. doi: 10.1128/AAC.01499-05.

Pagano M, Martins AF, Barth AL (2016) Mobile genetic elements related to carbapenem resistance in Acinetobacter baumannii. Braz J Microbiol. 47(4):785-792. doi: 10.1016/j.bjm.2016.06.005.

Paixão L, Rodrigues L, Couto I, Martins M, Fernandes P, De CarvalhoCC, Monteiro GA, Sansonetty F, Amaral L, Viveiros M (2009) Fluorometric determination of ethidium bromide efflux kinetics in Escherichia coli. J Biol Eng 3:18. doi: 10.1186/1754-1611-3-18.

Pankey GA (2005) Tigecycline. J Antimicrobial Chemotherapy 56:470-480. doi:10.1093/jac/dki248.

Paulsen IT, Brown MH, Skurray RA (1996) Proton-dependent multidrug efflux systems. Microbiol Rev. 60(4):575-608.

Paulsen IT, Brown MH, Littlejohn TG, Mitchell BA, Skurray RA (1996b) Multidrug resistance proteins QacA and QacB from Staphylococcus aureus: membrane topology and identification of residues involved in substrate specificity. Proc Natl Acad Sci U S A. 16;93(8):3630-5. doi: 10.1073/pnas.93.8.3630.

Peleg AY, Adams J, Paterson DL (2007) Tigecycline efflux as a mechanism for nonsusceptibility in Acinetobacter baumannii. Antimicrob Agents Chemother. 51:2065-2069. doi: 10.1128/AAC.0119806.

Peleg AY, Seifert H, Paterson DL (2008) Acinetobacter baumannii: emergence of a successful pathogen. Clin. Microbiol. Rev. 21:538-582. doi: 10.1128/CMR.00058-07.

Pérez-Varela M, Corral J, Aranda J, Barbé J (2019) Roles of efflux pumps from different superfamilies in the surface-associated motility and virulence of Acinetobacter baumannii ATCC 17978. Antimicrobial Agents Chemother. 63(3)e02190-18. doi: 10.1128/AAC.02190-18.

Petersen PJ, Jacobus NV, Weiss WJ, Sum PE, Testa RT (1999) In vitro and in vivo antibacterial activities of a novel glycylcyline, the 9-t-butylglycylamido derivative of minocycline (GAR-396). Antimicrob Agents Chemother 43(4):738-44.

Piddock LJ (2006) Clinically relevant chromosomally encoded multidrug resistance efflux pumps in bacteria. Clin Microbiol Rev. 19(2):382-402. doi: 10.1128/CMR.19.2.382-402.2006.

Poirel L and Nordmann P (2006) Carbapenem resistance in Acinetobacter baumannii: mechanisms and epidemiology. Clinical Microbiology and Infection 12: 826-836. doi: 10.1111/j.1469 0691.2006.01456.x.

Poirel L, Jayol A, Nordmann P (2017) Polymyxins: antibacterial activity, susceptibility testing, and resistance mechanisms encoded by plasmids or chromosomes. Clin Microbiol Rev. 30(2):557596. doi: 10.1128/CMR.00064-16. 
Poirel L, Menuteau O, Agoli N, Cattoen C, Nordmann P (2003) Outbreak of extended-spectrum betalactamase VEB-1-producing isolates of Acinetobacter baumannii in a French hospital. J Clin Microbiol.41(8):3542-7. doi: 10.1128/jcm.41.8.3542-3547.2003.

Poole K (2005) Efflux-mediated antimicrobial resistance. J Antimicrob Chemother. 56(1):20-51. doi: 10.1093/jac/dki171.

Pos KM (2009) Drug transport mechanism of the AcrB efflux pump. Biochim. Biophys. Acta 1794: 782793. doi: 10.1016/j.bbapap.2008.12.015.

Reddy VS, Shlykov MA, Castillo R, Sun El, Saier MH Jr (2012) The major facilitator superfamily (MFS) revisited. FEBS J. 279(11):2022-35. doi: 10.1111/j.1742-4658.2012.08588.x. Erratum in: FEBS J. 2013 Aug;280(16):3975.

Reyes N, Ginter C, Boudker O (2009) Transport mechanism of a bacterial homologue of glutamate transporters. Nature. 462(7275):880-5. doi: 10.1038/nature08616.

Ribera A, Roca I, Ruiz J, Gibert I, Vila J (2003a) Partial characterization of a transposon containing the $\operatorname{tet}(A)$ determinant in a clinical isolate of Acinetobacter baumannii. J. Antimicrob. Chemother. 52(3):477-480. doi: 10.1093/jac/dkg344.

Ribera A, Ruiz J, Vila J (2003b) Presence of the TetM determinant in a clinical isolate of Acinetobacter baumannii. Antimicrob Agents Chemother. 47(7):2310-2312. doi: 10.1128/aac.47.7.23102312.2003

Ribeiro da Cunha B, Fonseca LP, Calado CRC (2019) Antibiotic Discovery: Where Have We Come from, Where Do We Go? Antibiotics (Basel) 8(2):45. doi: 10.3390/antibiotics8020045.

Richmond GE, Evans LP, Anderson MJ, Wand ME, Bonney LC, Ivens A, Chua KL, Webber MA, Sutton JM, Peterson ML, Piddock LJ (2016) The Acinetobacter baumannii two-component system AdeRS regulates genes required for multidrug efflux, biofilm formation, and virulence in a strainspecific manner. mBio. 19;7(2):e00430-16. doi: 10.1128/mBio.00430-16. Erratum in: MBio. 7(3):e00852-16. doi: 10.1128/mBio.00852-16.

Roberts MC (2005) Update on acquired tetracycline resistance genes. FEMS Microbiol Lett. 245(2):195-203. doi: 10.1016/j.femsle.2005.02.034.

Robinson AE, Thomas NE, Morrison EA, Balthazor BM, Henzler-Wildman KA (2017) New freeexchange model of EmrE transport. Proc Natl Acad Sci U S A. 114(47):E10083-E10091. doi: 10.1073/pnas.1708671114.

Roca I, Marti S, Espinal P, Martínez P, Gibert I, Vila J (2009) CraA, a major facilitator superfamily efflux pump associated with chloramphenicol resistance in Acinetobacter baumannii. Antimicrob Agents Chemother. 53(9):4013-4014. doi: 10.1128/AAC.00584-09.

Rosenfeld N, Bouchier C, Courvalin P, Périchon B (2012) Expression of the resistance-nodulation-cell division pump AdelJK in Acinetobacter baumannii is regulated by AdeN, a TetR-type regulator. Antimicrob Agents Chemother. 56(5):2504-2510. doi: 10.1128/AAC.06422-11.

Rotem D and Schuldiner S (2004) EmrE, a multidrug transporter from Escherichia coli, transports monovalent and divalent substrates with the same stoichiometry. J Biol Chem. 279(47):48787-93. doi: 10.1074/jbc.M408187200. 
Rumbo C, Gato E, López M, Ruiz de Alegría C, Fernández-Cuenca F, Martínez-Martínez L, Vila J, Pachón J, Cisneros JM, Rodríguez-Baño J, Pascual A, Bou G, Tomás M (2013) Contribution of efflux pumps, porins, and $\beta$-lactamases to multidrug resistance in clinical isolates of Acinetobacter baumannii. Antimicrob Agents Chemother. 57(11): 5247-5257. doi: 10.1128/AAC.00730-13.

Rumbo C, Tomás M, Fernández Moreira E, Soares NC, Carvajal M, Santillana E, Beceiro A, Romero A, Bou G (2014) The Acinetobacter baumannii Omp33-36 porin is a virulence factor that induces apoptosis and modulates autophagy in human cells. Infect. Immun. 82:4666-4680. doi: 10.1128/IAI.02034-14.

Ruzin A, Immermann FW, Bradford PA (2010) RT-PCR and statistical analyses of adeABC expression in clinical isolates of Acinetobacter calcoaceticus-Acinetobacter baumannii complex. Microb. Drug Resist. 16:87-89. doi: 10.1089/mdr.2009.0131.

Ruzin A, Keeney D, Bradford PA (2007) AdeABC multidrug efflux pump is associated with decreased susceptibility to tigecycline in Acinetobacter calcoaceticus-Acinetobacter baumannii complex. J. Antimicrob. Chemother. 59:1001-1004. doi: 10.1093/jac/dkm058.

Ruzin A, Visalli MA, Keeney D, Bradford PA (2005) Influence of transcriptional activator RamA on expression of multidrug efflux pump AcrAB and tigecycline susceptibility in Klebsiella pneumoniae. Antimicrob. Agents Chemother. 49:1017-1022. doi: 10.1128/AAC.49.3.1017-1022.2005.

Ryan RM and Vandenberg RJ (2016) Elevating the alternating-access model. Nat Struct Mol Biol. 23(3):187-9. doi: 10.1038/nsmb.3179.

Santos-Lopez A, Marshall CW, Scribner MR, Snyder DJ, Cooper VS (2019) Evolutionary pathways to antibiotic resistance are dependent upon environmental structure and bacterial lifestyle. Elife. 8:e47612. doi: 10.7554/eLife.47612.

Saranathan R, Pagal S, Sawant AR, Tomar A, Madhangi M, Sah S, Satti A, Arunkumar KP, Prashanth K (2017) Disruption of tetR type regulator adeN by mobile genetic element confers elevated virulence in Acinetobacter baumannii. Virulence 8(7):1316-1334. doi: 10.1080/21505594.2017.1322240.

Sato Y, Unno Y, Ubagai T, Ono Y (2018) Sub-minimum inhibitory concentrations of colistin and polymyxin B promote Acinetobacter baumannii biofilm formation. PLoS One. 19;13(3):e0194556. doi: 10.1371/journal.pone. 0194556.

Schwarz S, Kehrenberg C, Doublet B, Cloeckaert A (2004) Molecular basis of bacterial resistance to chloramphenicol and florfenicol. FEMS Microbiology Reviews 28(5): 519-542. doi: 10.1016/j.femsre.2004.04.001.

Schwarz S, Shen J, Kadlec K, Wang Y, Brenner Michael G, Feßler AT, Vester B (2016) Lincosamides, Streptogramins, Phenicols, and Pleuromutilins: Mode of Action and Mechanisms of Resistance. Cold Spring Harb Perspect Med. 6(11):a027037. doi: 10.1101/cshperspect.a027037.

Seeger MA, Schiefner A, Eicher T, Verrey F, Diederichs K, Pos KM (2006) Structural asymmetry of AcrB trimer suggests a peristaltic pump mechanism. Science 313: 1295-1298. doi: 10.1126/science. 1131542 . 
Sheng ZK, Hu F, Wang W, Guo Q, Chen Z, Xu X, Zhu D, Wang M (2014) Mechanisms of tigecycline resistance among Klebsiella pneumoniae clinical isolates. Antimicrob Agents Chemother. 58(11):6982-6985. doi: 10.1128/AAC.03808-14.

Sigal N, Molshanski-Mor S, Bibi E (2006) No single irreplaceable acidic residues in the Escherichia coli secondary multidrug transporter MdfA. J Bacteriol. 188(15):5635-9. doi: 10.1128/JB.00422-06.

Sigal N, Fluman N, Siemion S, Bibi E (2009) The secondary multidrug/proton antiporter MdfA tolerates displacements of an essential negatively charged side chain. J Biol Chem. 284(11):6966-71. doi: 10.1074/jbc.M808877200.

Siroy A, Molle V, Lemaître-Guillier C, Vallenet D, Pestel-Caron M, Cozzone AJ, Jouenne T, Dé E (2005) Channel formation by CarO, the carbapenem resistance-associated outer membrane protein of Acinetobacter baumannii. Antimicrob Agents Chemother. 49(12):4876-83. doi: 10.1128/ AAC.49.12.4876-4883.2005.

Smani Y and Pachón J (2013) Loss of the OprD homologue protein in Acinetobacter baumannii: impact on carbapenem susceptibility. Antimicrob Agents Chemother. 57(1):677. doi: 10.1128/AAC.01277-12.

Smani Y, Dominguez-Herrera J, Pachón J (2013) Association of the outer membrane protein Omp33 with fitness and virulence of Acinetobacter baumannii. J. Infect. Disease 208:1561-1570. doi: 10.1093/infdis/jit386.

Smani Y, Fabrega A, Roca I, Sanchez-Encinales V, Vila J, Pachon J (2014) Role of OmpA in the multidrug resistance phenotype of Acinetobacter baumannii. Antimicrob. Agents Chemother. 58(3):1806-1808. doi: 10.1128/AAC.02101-13.

Someya Y, Yamaguchi A, Sawai T (1995) A novel glycylcycline, 9-(N,N-dimethylglycylamido)-6demethyl-6-deoxytetracycline, is neither transported nor recognized by the transposon Tn10encoded metal-tetracycline/ $\mathrm{H}+$ antiporter. Antimicrob Agents Chemother. 39(1):247-249. doi: 10.1128/aac.39.1.247.

Speer BS, Bedzyk L, Salyers AA (1991) Evidence that a novel tetracycline resistance gene found on two Bacteroides transposons encodes an NADP-requiring oxidoreductase. J Bacteriol. 173(1):176-83. doi: 10.1128/jb.173.1.176-183.1991.

Srinivasan VB, Rajamohan G, Gebreyes WA (2009) Role of AbeS, a novel efflux pump of the SMR family of transporters, in resistance to antimicrobial agents in Acinetobacter baumannii. Antimicrobial Agents and Chemother. 53(12):5312-5316. doi: 10.1128/AAC.00748-09.

Stahl J, Bergmann H, Göttig S, Ebersberger I, Averhoff B (2015) Acinetobacter baumannii Virulence Is Mediated by the Concerted Action of Three Phospholipases D. PLoS One.10(9):e0138360. doi: 10.1371/journal.pone.0138360.

Stark MJ (1987) Multicopy expression vectors carrying the lac repressor gene for regulated high-level expression of genes in Escherichia coli. Gene 51: 255-267. doi:10.1016/0378-1119(87)90314-3.

Su XZ, Chen J, Mizushima T, Kuroda T, Tsuchiya T (2005) AbeM, an $\mathrm{H}^{+}$-coupled Acinetobacter baumannii multidrug efflux pump belonging to the MATE family of transporters. Antimicrob Agents Chemother. 49(10):4362-4364. doi: 10.1128/AAC.49.10.4362-4364.2005. 
Sugawara E and Nikaido H (2012) OmpA is the principal nonspecific slow porin of Acinetobacter baumannii. J. Bacteriol. 194(15):4089-4096. doi: 10.1128/JB.00435-12.

Sugawara E, Steiert M, Rouhani S, Nikaido H (1996) Secondary structure of the outer membrane proteins OmpA of Escherichia coli and OprF of Pseudomonas aeruginosa. J. Bacteriol. 178:60676069. doi: 10.1128/jb.178.20.6067-6069.1996.

Sum PE, Lee VJ, Testa RT, Hlavka JJ, Ellestad GA, Bloom JD, Gluzman Y, and Tally FP (1993) Glycylcyclines. 1. A new generation of potent antibacterial agents through modification of 9aminotetracyclines. J. Med. Chem. 37:184-188. doi: 10.1021/jm00027a023.

Sun J, Chen C, Cui CY, Zhang Y, Liu X, Cui ZH, Ma XY, Feng Y, Fang LX, Lian XL, Zhang RM, Tang YZ, Zhang KX, Liu HM, Zhuang ZH, Zhou SD, Lv JN, Du H, Huang B, Yu FY, Mathema B, Kreiswirth BN, Liao XP, Chen L, Liu YH (2019) Plasmid-encoded tet $(X)$ genes that confer highlevel tigecycline resistance in Escherichia coli. Nat Microbiol. 4(9):1457-1464. doi: 10.1038/s41564-019-0496-4.

Sun JR, Jeng WY, Perng CL, Yang YS, Soo PC, Chiang YS, Chiueh TS (2016) Single amino acid substitution Gly186Val in AdeS restores tigecycline susceptibility of Acinetobacter baumannii. J Antimicrobial Chemother. 71(6):1488-1492. doi: 10.1093/jac/dkw002

Sun JR, Perng CL, Chan MC, Morita Y, Lin JC, Su CM, Wang WY, Chang TY, Chiueh TS (2012) A truncated AdeS kinase protein generated by ISAba1 insertion correlates with tigecycline resistance in Acinetobacter baumannii. PLoS One 7(11):e49534. doi: 10.1371/journal.pone.0049534.

Tal N and Schuldiner S (2009) A coordinated network of transporters with overlapping specificities provides a robust survival strategy. Proc Natl Acad Sci USA 106:9051-9056. doi: 10.1073/pnas.0902400106.

Tanaka Y, Hipolito CJ, Maturana AD, Ito K, Kuroda T, Higuchi T, Katoh T, Kato HE, Hattori M, Kumazaki K, Tsukazaki T, Ishitani R, Suga H, Nureki O (2013) Structural basis for the drug extrusion mechanism by a MATE multidrug transporter. Nature 496:247-251. doi: 10.1038/nature12014.

Thomas C and Tampé R (2020) Structural and Mechanistic Principles of ABC Transporters. Annu Rev Biochem. 89:605-636. doi: 10.1146/annurev-biochem-011520-105201.

Tirosh O, Sigal N, Gelman A, Sahar N, Fluman N, Siemion S, Bibi E (2012) Design of new Mdr transporter capabilities. Proc Natl Acad Sci USA 109(31):12473-12478. doi: 10.1073/pnas.1203632109.

Trias J and Nikaido H (1990) Outer membrane protein D2 catalyzes facilitated diffusion of carbapenems and penems through the outer membrane of Pseudomonas aeruginosa. Antimicrob. Agents Chemother. 34:52-57. doi: 10.1128/aac.34.1.52.

Trott O, Olson AJ (2010) AutoDock Vina: improving the speed and accuracy of docking with a new scoring function, efficient optimization, and multithreading. J Comput Chem. 31(2):455-61. doi: $10.1002 / j c c .21334$. 
Tseng TT, Gratwick KS, Kollman J, Park D, Nies DH, Goffeau A, Saier MH Jr (1999) The RND permease superfamily: an ancient, ubiquitous and diverse family that includes human disease and development proteins. J Mol Microbiol Biotechnol. 1(1):107-25.

Tuckman M, Petersen PJ, Projan SJ (2000) Mutations in the interdomain loop region of the $\operatorname{tet} A(A)$ tetracycline resistance gene increase efflux of minocycline and glycylcyclines. Microb Drug Resist 6:277-282. doi: 10.1089/mdr.2000.6.277.

Valencia R, Arroyo LA, Conde M, Aldana JM, Torres MJ, Fernández-Cuenca F, Garnacho-Montero J, Cisneros JM, Ortíz C, Pachón J, Aznar J (2009) Nosocomial outbreak of infection with pan-drugresistant Acinetobacter baumannii in a tertiary care university hospital. Infect Control Hosp Epidemiol. 30: 257-63. doi: 10.1086/595977.

Vila J, Martí S, Sánchez-Céspedes J (2007) Porins, efflux pumps and multidrug resistance in Acinetobacter baumannii. J Antimicrobial Chemother. 59(6):1210-1215. doi: 10.1093/jac/dkl509.

Vila J, Marcos A, Marco F, Abdalla S, Vergara Y, Reig R, Gomez-Lus R, Jimenez de Anta T (1993) In vitro antimicrobial production of beta-lactamases, aminoglycoside-modifying enzymes, and chloramphenicol acetyltransferase by and susceptibility of clinical isolates of Acinetobacter baumannii. Antimicrob Agents Chemother. 37(1):138-41. doi: 10.1128/aac.37.1.138.

Wang L, Liu D, Lv Y, Cui L, Li Y, Li T, Song H, Hao Y, Shen J, Wang Y, Walsh TR (2020) Novel plasmid-mediated tet(X5) gene conferring resistance to tigecycline, eravacycline, and omadacycline in a clinical Acinetobacter baumannii isolate. Antimicrob Agents Chemother 64:e01326-19. doi: 10.1128/AAC.01326-19.

Wang P, McElheny CL, Mettus RT, Shanks RMQ, Doi Y (2017) Contribution of the TetB efflux pump to minocycline susceptibility among carbapenem-resistant Acinetobacter baumannii strains. Antimicrob Agents Chemother. 61(10):e01176-17. doi: 10.1128/AAC.01176-17.

Wang Z, Fan G, Hryc CF, Blaza JN, Serysheva II, Schmid MF, Chiu W, Luisi BF, Du D (2017) An allosteric transport mechanism for the AcrAB-ToIC multidrug efflux pump. Elife. 6:e24905. doi: 10.7554/eLife.24905.

Wen Y, Ouyang Z, Yu Y, Zhou X, Pei Y, Devreese B, Higgins PG, Zheng F (2017) Mechanistic insight into how multidrug resistant Acinetobacter baumannii response regulator AdeR recognizes an intercistronic region. Nucleic Acids Res. 19;45(16):9773-9787. doi: 10.1093/nar/gkx624.

West AH and Stock AM (2001) Histidine kinases and response regulator proteins in two-component signaling systems. Trends Biochem Sci. 26:369-376. doi: 10.1016/s0968-0004(01)01852-7.

World Health Organization (2017) Global priority list of antibiotic resistant bacteria to guide research, discovery, and development of new antibiotics. https://www.who.int/news/item/27-02-2017-whopublishes-list-of-bacteria-for-which-new-antibiotics-are-urgently-needed

Wisplinghoff H, Decker M, Haefs C, Krut O, Plum G, Seifert H (2003) Mutations in gyrA and parC associated with resistance to fluoroquinolones in epidemiologically defined clinical strains of Acinetobacter baumannii. J. Antimicrob. Chemother. 51:177-180. doi: 10.1093/jac/dkf254.

Woodall NB, Hadley S, Yin Y, Bowie JU (2017) Complete topology inversion can be part of normal membrane protein biogenesis. Protein Sci 26:824-833. doi: 10.1002/pro.3131. 
Wu HH, Symersky J, Lu M (2019) Structure of an engineered multidrug transporter MdfA reveals the molecular basis for substrate recognition. Commun Biol 2, 210. doi: 10.1038/s42003-019-0446-y.

Wu HH, Symersky J, Lu M (2020) Structure and mechanism of a redesigned multidrug transporter from the Major Facilitator Superfamily. Sci Rep 10, 3949. doi: 10.1038/s41598-020-60332-8.

Yamaguchi A, Ono N, Akasaka T, Noumi T and Sawai T (1990) Metal-tetracycline/ $\mathrm{H}^{+}$antiporter of Escherichia coli encoded by a transposon, Tn10. J. Biol. Chem. 265: 15525-15530.

Yan N (2015) Structural biology of the Major Facilitator Superfamily transporters. Annu. Rev. Biophys. 44:257-83. doi: 10.1146/annurev-biophys-060414-033901.

Yang J, Zhang $Y$ (2015) I-TASSER server: new development for protein structure and function predictions. Nucleic Acids Res. 43(W1):W174-81. doi: 10.1093/nar/gkv342.

Yang W, Moore IF, Koteva KP, Bareich DC, Hughes DW, Wright GD (2004) TetX is a flavin-dependent monooxygenase conferring resistance to tetracycline antibiotics. J Biol Chem. 10:279(50): 5234652. doi: $10.1074 / j b c . M 409573200$.

Yardeni EH, Zomot E, Bibi E (2018) The fascinating but mysterious mechanistic aspects of multidrug transport by MdfA from Escherichia coli. Res Microbiol. 169(7-8):455-460. doi: 10.1016/j.resmic.2017.09.004.

Yardeni EH, Mishra S, Stein RA, Bibi E, Mchaourab HS (2020) The multidrug transporter MdfA deviates from the canonical model of alternating access of MFS transporters. J Mol Biol. 432(20):5665-5680. doi: 10.1016/j.jmb.2020.08.017.

Yoon EJ, Chabane YN, Goussard S, Snesrud E, CourvalinP, Dé E, Grillot-Courvalin C (2015) Contribution of Resistance-Nodulation-Cell Division efflux systems to antibiotic resistance and biofilm formation in Acinetobacter baumannii. mBio 6(2):e00309-15. doi: 10.1128/mBio.00309-15.

Yoon EJ, Courvalin P, Grillot-Courvalin C (2013) RND-type efflux pumps in multidrug-resistant clinical isolates of Acinetobacter baumannii: major role for AdeABC overexpression and AdeRS mutations. Antimicrob Agents Chemother. 57:2989-2995. doi: 10.1128/AAC.02556-12.

Zahn M, Bhamidimarri SP, Baslé A, Winterhalter M, van den Berg B (2016) Structural insights into outer membrane permeability of Acinetobacter baumannii. Structure 24(2):221-231. doi: 10.1016/j.str.2015.12.009.

Zahn M, D'Agostino T, Eren E, Baslé A, Ceccarelli M, van den Berg B (2015) Small molecule transport by CarO, an abundant eight-stranded $\beta$-barrel outer membrane protein from Acinetobacter baumannii. Journal of Molecular Biology. 427(14):2329-2339. doi: 10.1016/j.jmb.2015.03.016.

Zgurskaya HI, Löpez CA, Gnanakaran S (2015) Permeability barrier of Gram-negative cell envelopes and approaches to bypass It. ACS Infect Dis. 1(11):512-522. doi: 10.1021/acsinfecdis.5b00097.

Zhang R, Dong N, Zeng Y, Shen Z, Lu J, Liu C, Huang Z, Sun Q, Cheng Q, Shu L, Cai J, Chan EW, Liu D, Chen G, Wang Y, Chen S (2020) Chromosomal and plasmid-borne tigecycline resistance genes tet $(\mathrm{X} 3)$ and tet $(\mathrm{X} 4)$ in dairy cows on a Chinese farm. Antimicrob Agents Chemother 64:e00674-20. doi: 10.1128/AAC.00674-20. 
Zhao Q, Li XZ, Srikumar R, Poole K (1998) Contribution of outer membrane efflux protein OprM to antibiotic resistance in Pseudomonas aeruginosa independent of MexAB. Antimicrob. Agents Chemother. 42:1682-1688.

Zhou H, Zhang T, Yu D, Pi B, Yang Q, Zhou J, Hu S, Yu Y (2011) Genomic analysis of the multidrugresistant Acinetobacter baumannii strain MDR-ZJ06 widely spread in China. Antimicrob Agents Chemother. 55(10):4506-12. doi: 10.1128/AAC.01134-10.

Zhu Y, Lu J, Zhao J, Zhang X, Yu HH, Velkov T, Jian Li (2020) Complete genome sequence and genome-scale metabolic modelling of Acinetobacter baumannii type strain ATCC 19606. International Journal of Medical Microbiology 310:151412. doi: 10.1016/j.ijmm.2020.151412.

Zomot E, Yardeni EH, Vargiu AV, Tam HK, Malloci G, Ramaswamy VK, Perach M, Ruggerone P, Pos $\mathrm{KM}$, Bibi E (2018) A new critical conformational determinant of multidrug efflux by an MFS Transporter. J Mol Biol. 430(9):1368-1385. doi: 10.1016/j.jmb.2018.02.026. 


\section{ABBREVIATIONS}

$\mathrm{ABC}$

ATP-Binding Cassette

ACMA

9-amino-6-chloro-2-methoxyacridine

APS

Ammonium persulfate

ATCC

American Type Culture Collection

ATP

Adenosine triphosphate

BCIP

5-bromo-4-chloro-3-indolylphosphate

bp

base pair

BSA

Bovine Serum Albumin

CCCP

Carbonyl cyanide m-chlorophenylhydrazone

$\mathrm{C}_{\mathrm{T}}$

Cycle threshold

DAPI

4',6-Diamidino-2-phenylindole

$\mathrm{ddH}_{2} \mathrm{O}$

double-distilled water

DHA

drug/ $\mathrm{H}^{+}$antiporter (DHA)

DNA

Deoxyribonucleic acid

DNase I

Deoxyribonuclease I

dNTPs

deoxynucleotide triphosphates

DTT

1,4-dithiothreitol

EDTA Ethylendiaminetetracetic acid

IM

Inner membrane

IPTG

Isopropyl- $\beta$-D-thiogalactopyranosid

IS

Insertion sequence

Kan

Kanamycin

$\mathrm{kb}$

kilo base

$\mathrm{kDa}$

kilodalton

$\mathrm{KPi}$

Potassium phosphate (buffer)

LB

Lysogeny broth

LPS Lipopolysaccharide

$\mathrm{mA} \quad$ Milliampere

$\min \quad$ Minutes

MATE Multidrug and Toxic Compound Extrusion

MFS Major Facilitator Superfamily

MIC Minimal Inhibitory Concentration

NAD Nicotinamide adenine dinucleotide

NADPH Nicotinamide adenine dinucleotide phosphate 


\begin{tabular}{|c|c|}
\hline NBD & Nucleotide Binding Domain \\
\hline NBT & Nitro-blue tetrazolium \\
\hline NMR & Nuclear Magnetic Resonance \\
\hline $\mathrm{OD}_{600}$ & Optical Density at $600 \mathrm{~nm}$ \\
\hline OM & Outer Membrane \\
\hline ORF & Open reading frame \\
\hline PACE & Protobacterial Antimicrobal Compound Efflux \\
\hline PDB & Protein Data Bank \\
\hline PCR & Polymerase Chain Reaction \\
\hline RNA & Ribonucleic acid \\
\hline RND & Resistance-Nodulation-cell Division \\
\hline rpm & Revolutions per minute \\
\hline RPPs & Ribosomal Protection Proteins \\
\hline RT-qPRC & Reverse Transcription Quantitative PCR \\
\hline s & Seconds \\
\hline SBP & Substrate Binding Protein \\
\hline SDS & Sodium Dodecyl Sulfate \\
\hline SDS-PAGE & SDS-Polyacrylamide Gel Electrophoresis \\
\hline SMR & Small Multidrug Resistance \\
\hline SOC & Super Optimal broth with Catabolite repression \\
\hline TEMED & N,N,N',N'-tetramethylethylendiamine \\
\hline TM & Transmembrane helix \\
\hline $\mathrm{TPP}^{+}$ & Tetraphenylphosphonium \\
\hline TRIS & Tris-hydroxymethyl-aminomethane (buffer) \\
\hline V & Volt \\
\hline UV & Ultraviolet \\
\hline WT & Wild-type \\
\hline
\end{tabular}




\section{DECLARATION}

Except where stated otherwise by reference or acknowledgment, the work presented was generated by myself under the supervision of my advisors during my doctoral studies. All contributions from colleagues are explicitly referenced in the thesis. The material listed below was obtained in the context of collaborative research:

- None

Whenever a Figure or table is identical to a previous publication, it is stated explicitly in the thesis that copyright permission and/or co-author agreement has been obtained. The following parts of the thesis have been previously published:

Foong WE, Tam HK, Crames JJ, Averhoff B, Pos KM (2019) The chloramphenicol/ $\mathrm{H}^{+}$ antiporter CraA of Acinetobacter baumannii AYE reveals a broad substrate specificity. J. Antimicrob. Chemother. 74: 1192-1201.

- Chapter 3.3 Characterization of CraA

- Figure 3.13 Drug susceptibility assays of $E$. coli deletion mutants harbouring craA (WT or variants of membrane-embedded titratable residues).

- Figure 3.18 Dequalinium transport assay in whole cell conducted using $E$. coli BW25113 $\triangle$ emrE $\triangle m d f A \triangle a c r A B$ overexpressing $c r a A$ wild-type or variants.

- Figure 3.19 Accumulation of dequalinium in E. coli BW25113 $\Delta$ emrE $\Delta m d f A \Delta a c r A B$ harbouring pTTQ18, pTTQ18_CraA wild-type and variants.

- Figure 3.20 Ethidium efflux catalyzed by CraA.

- Figure 3.30 Proton-coupled substrate transport by inverted membrane vesicles.

- Figure 3.31 Drug susceptibility assays of wild-type A. baumannii ATCC 19606 (WT), $\triangle c r a A$ and $\triangle c r a A$ complemented with pBAV1K_CraA.

Foong WE, Wilhelm J, Tam HK, Pos KM (2020) Tigecycline efflux in Acinetobacter baumannii is mediated by TetA in synergy with RND-type efflux transporters. J. Antimicrob. Chemother. 75: 1135-1139.

- Chapter 3.5 TetA of $A$. baumannii strain AYE and tigecycline resistance

- Figure 3.9 Drug susceptibility assays of E. coli BW25113 $\Delta$ emrE $\Delta m d f A$ harbouring putative chloramphenicol transporter genes from A. baumannii strain AYE.

- Table 3.3 MICs of tetracyclines for E. coli harbouring empty vector, pTTQ18_TetA, or pTTQ18_TetG.

- Figure 3.37 Drug susceptibility assay of $A$. baumannii ATCC 19606 WT and RNDknockout strains complemented with pBAV1K_TetA.

- Table 3.4 MIC values of tigecycline for different strains of $A$. baumannii.

- Figure 3.38 Differential gene analysis of tet $A$ and RND efflux pump genes in $A$. baumannii AYE in the absence or presence of tigecycline. 


\section{ACKNOWLEDGEMENT}

Without the encouragement and participation of many people, this project would never have been accomplished.

My deepest appreciation goes to my supervisor, Prof. Dr. Klaas Martinus Pos for giving me the chance to join his lab for my master thesis and my PhD study. He provides guidance and support throughout my study, as well as giving me freedom to explore my field of interest. In addition, I would like to express my gratitude to all the lab members of Pos lab, especially Andrea Herrmann who provides help for me in the lab or in daily life.

I would like to thank the DFG Research Unit FOR 2251 'Adaptation and persistence of the emerging pathogen Acinetobacter baumannii' for the financial support. I appreciate the fellow students in the research unit for sharing their experience in their projects during the annual meeting, and the principle investigators for their insightful comments and suggestions on my project.

I would like to express my sincere thanks to Prof. Dr. Beate Averhoff for her generosity in providing S2 lab facility and bacterial strains for my experiments related to A. baumannii. Her expertise in the field of $A$. baumannii and her guidance in creating knockout strains of $A$. baumannii are very much appreciated. My sincere thanks is also extended to the lab members of Prof. Dr. Beate Averhoff and Prof. Dr. Volker Müller, including Dr. Sabine Zeidler, Josephine Hubloher and Jennifer Breisch for providing assistance to me during my experiments in S2 lab.

A very special thanks to the former bachelor student, Stephanie Himpich, who helped me in German translation of the summary part of this thesis.

Last but not the least, I would like to thank my husband Heng Keat Tam. Without his guidance and encouragement in the past few years, it would be impossible for me to complete my study. 


\section{Curriculum vitae}

Name: Foong, Wuen Ee

Date of birth : 22.12.1982

Place of birth: Kuala Lumpur, Malaysia

Education:

07/2016 - present : $\quad$ PhD candidate in Institute of Biochemistry, Goethe University Frankfurt, Germany

09/2013 - 11/2015 : Master degree in Agrobiotechnology, Justus-Liebig University Giessen, Germany

01/2007-01/2008 : Certificate in Education, Teacher Training College, Penang, Malaysia

09/2003 - 09/2006 : Bachelor degree in Biotechnology, University Malaysia Sabah, Malaysia

Conference / Workshop attended:

$14-15.11 .2016$

Workshop: De-Mystifying X-ray Data Processing in Macromolecular Crystallography, Charles Darwin House, London.

$02-03.03 .2017$

Poster presentation in Acinetobacter baumannii International Meeting 2017, Goethe University Frankfurt.

$17-20.03 .2019$

Poster presentation in Annual Conference 2019 of the Association for General and Applied Microbiology (VAAM), Johannes Gutenberg University Mainz.

$04-06.09 .2019$

Poster presentation in the $12^{\text {th }}$ International Symposium on the Biology of Acinetobacter 2019, Goethe University Frankfurt.

Publications:

Foong WE, Wilhelm J, Tam HK, Pos KM (2020) Tigecycline efflux in Acinetobacter baumannii is mediated by TetA in synergy with RND-type efflux transporters. J. Antimicrob. Chemother. 75: 1135-1139.

Foong WE, Tam HK, Crames JJ, Averhoff B, Pos KM (2019) The chloramphenicol/ $\mathrm{H}^{+}$ antiporter CraA of Acinetobacter baumannii AYE reveals a broad substrate specificity. J. Antimicrob. Chemother. 74: 1192-1201.

Tam HK, Malviya VN, Foong WE, Herrmann A, Malloci G, Ruggerone P, Vargiu AV, Pos KM (2020) Binding and transport of carboxylated drugs by the multidrug transporter AcrB. J. Mol. Biol. 432: 861-877. 